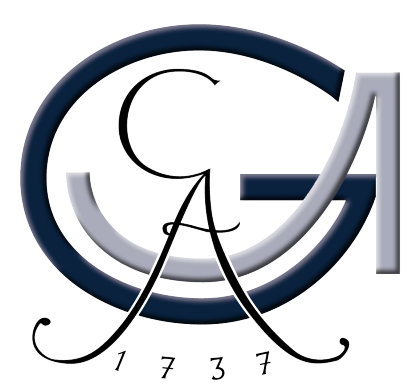

\title{
Investigation of DNA in Cell Nuclei by Combined Imaging Techniques
}

\author{
Dissertation \\ for the award of the degree \\ "Doctor of Philosophy" \\ of the Georg-August-Universität Göttingen
}

within the doctoral program

Göttingen Graduate School for Neurosciences, Biophysics

and Molecular Biosciences (GGNB)

of the Georg-August-University School of Science (GAUSS)

submitted by

\section{Andrew Nicholas Wittmeier}

from Hemet (California, U.S.A.)

Göttingen, Germany

2020 



\section{Members of the Thesis Committee:}

Prof. Dr. Sarah Köster (Reviewer)

Institute for X-ray Physics

Georg-August-Universität Göttingen

Prof. Dr. Ralf Ficner (Reviewer)

Institute for Microbiology and Genetics

Georg-August-Universität Göttingen

Dr. Alexander Egner

Laser Laboratory, Göttingen

\section{Further Members of the Examination Board:}

Prof. Dr. Tim Salditt

Institute for X-ray Physics

Georg-August-Universität Göttingen

Prof. Dr. Stefan Klumpp

Institute for Dynamics of Complex Systems

Georg-August-Universität Göttingen

Dr. Gražvydas Lukinavičius

Max Planck Institute for Biophysical Chemistry, Göttingen

\section{Date of Oral Examination:}

$10 / 11 / 2020$ 



\section{Acknowledgements}

This thesis is the manifestation of what I have learned during my doctoral studies at the Institute for X-Ray Physics. Needless to say, I have learned a lot throughout my journey and am incredibly grateful to those who have taught and helped me. In particular, I would like to express my sincerest gratitude to Prof. Dr. Sarah Köster, without whom this thesis would not have been possible. Sarah, I thank you for providing constant guidance and for always being available for help. I am very happy to have such a gifted scientist as my supervisor and I have learned a lot from you. Thank you. I would also like thank Prof. Dr. Tim Salditt for always taking the time to discuss the finer details of X-ray imaging with me, for help at the beamline and for refereeing my thesis defense. I would also like to thank Prof. Dr. Ralf Ficner for co-supervising my thesis, as well as Dr. Alex Egner, Prof. Dr. Stefan Klumpp and Dr. Gražvydas Lukinavičius for refereeing my defense. The success of this thesis was made possible by the assistance of Susanne Bauch, Jochen Herbst and Jan Goeman, who helped me prepare biological samples and solve IT problems. Thank you. Furthermore, I would like to thank Markus Osterhoff for IT assistance, help at the beamline and fruitful discussions. I would also like to thank Bastian Hartmann and Peter Luley for technical assistance while at the beamline. For all of the administrative help that I have received, I would like to thank Christine WilkeFeist, Kerstin Pluschke, Michaela Ständer, Eva Hetzel and Sabine Balder.

To the group in general, I thank you for making my journey so memorable and enjoyable! Chiara, thank you for all of our discussions and for your help during the beamtimes and in the lab. Eleonora, I thank you for always being available for a coffee and for placing bets that you would always lose. "Double or nothing?" A big shout-out to Gerrit for always helping me solve problems both inside and outside the university. Sup, Anička? Thanks, mama Julia, for all the cooking tips and amazing food! Jan-Philipp, thank you for always helping me with coding. Manuela, thank you for all of our fruitful discussions and for being part of the X-ray crew! Thank you, Lotta, for always being positive and for being everyone's cheerleader. Thanks, Anna S., for the fun discussions during our walks! Thank you Laura, Magdalena, Anna B., Jana, Ash, Viktor, Clément, Kim, Ulrike and Kamila for creating a such a great working environment! A special thanks goes out to Marten who helped me with, well, everything. All of those long nights paid off! I would also like to thank Marina, Anna-Lena, Marius, Johannes and Mareike for all of their help with holographic imaging. I would like to thank the GGNB and IMPRS for Physics of Biological and Complex Systems, in particular Frauke Bergmann and Antje Erdmann, for providing such a well-structured doctoral program. I would also like to thank Francesco Rocca of the Laser Lab for all of the help I received, it is greatly appreciated. 
Furthermore, I would like to thank my parents, Eric and Carol, for everything they have done. You two have provided me with every opportunity to pursue my education and have done so with loving and open arms. I am forever grateful for you two. Thank you. Last, but definitely not least, I want to thank Heidi Somsel, with whom I have shared the past four years of my life with. Heidi, thank you for your perpetual support and encouragement over the years, even while at Northern Arizona University. 
Contents

Acknowledgements $\quad$ i

List of Figures vii

List of Tables $\quad$ xi

1 Introduction 1

2 State of the Art 3

3 Light-Matter Interactions $\quad 7$

3.1 Optical Image Formation $\ldots \ldots \ldots \ldots$

3.2 Visible-Light Fluorescence Microscopy . . . . . . . . . . . . . . . . . . . . 9

3.2.1 Basic Principles . . . . . . . . . . . . . . . . . 10

3.2 .2 Epifluorescence Microscopy . . . . . . . . . . . . . . . . . . 12

3.2 .3 Confocal Microscopy . . . . . . . . . . . . . . . . . . . . . 13

3.2.4 Stimulated Emission Depletion Microscopy . . . . . . . . . . . . 15

3.3 X-Ray-Matter Interactions . . . . . . . . . . . . . . . . . . . . . . 19

3.3.1 Photon Cross-Section . . . . . . . . . . . . . . . . . 21

3.3.2 Photoelectric Effect . . . . . . . . . . . . . . . . 22

3.3 .3 Compton Scattering . . . . . . . . . . . . . . . . 23

3.3 .4 Thomson Scattering . . . . . . . . . . . . . . . 25

3.3.5 Electron-Positron Pair-Production . . . . . . . . . . . . 26

3.3 .6 Rayleigh Scattering . . . . . . . . . . . . . . . . 26

3.4 Kinematic Theory of Diffraction _. . . . . . . . . . . . 27

3.4 .1 Form Factor . . . . . . . . . . . . . . . . . . . . 29

3.4 .2 Structure Factor . . . . . . . . . . . . . . . . . 30

4 Experimental Methods 33

4.1 Sample Preparation . . . . . . . . . . . . . . . . . . . 33

4.1.1 Cell Culture on Silicon-Nitride Membranes . . . . . . . . . . . 33

4.1 .2 Fluorescent Labeling of DNA . . . . . . . . . . . . . . . . 35

4.1.3 Grid Plunging and Lyophilization . . . . . . . . . . . . . . 36

4.2 Small-Angle X-ray Scattering . . . . . . . . . . . . . . . . 38

4.2 .1 Contrast Formation in SAXS . . . . . . . . . . . . . 38 
4.2 .2 Porod's Law . . . . . . . . . . . . . . . . . . . . . 39

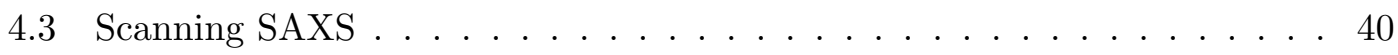

4.3.1 Experimental Setup at P10: Scanning SAXS . . . . . . . . . . . 41

4.3.2 Data Analysis: Scanning SAXS . . . . . . . . . . . . . . . . 42

4.4 X-ray Holography . . . . . . . . . . . . . . . . . . . . . . . . 44

4.4.1 Direct Reconstruction: Contrast Transfer Function . . . . . . . . . 45

4.4.2 Iterative Reconstruction: Relaxed Averaged Alternating Reflections 48

4.4.3 Experimental Setup at P10: Holography . . . . . . . . . . . . . 50

4.4.4 Data Analysis: X-ray Holography . . . . . . . . . . . . . . . . 51

4.5 X-ray Computed Tomography . . . . . . . . . . . . . . . 53

4.5.1 Experimental Setup at P10: Tomography . . . . . . . . . . 56

4.5.2 Data Analysis: X-ray Tomography . . . . . . . . . . . . 58

4.6 A Beamline-Compatible STED Microscope . . . . . . . . . . . . . . . 61

5 Combined Scanning Small Angle X-ray Scattering and Holography Probes Multiple Length Scales in Cell Nuclei $\quad 65$

5.1 Introduction . . . . . . . . . . . . . . . . . . . . . 65

5.2 Materials and Methods . . . . . . . . . . . . . . . . . . 67

5.2 .1 Experimental Setups . . . . . . . . . . . . . . . 68 68

5.2 .2 Data Analysis . . . . . . . . . . . . . . . . . . . . . . . . . . . . . . . . . . . . .

5.3 Results and Discussion . . . . . . . . . . . . . . . . . . 72

5.3.1 Aggregation State of DNA in Cell Nuclei . . . . . . . . . . . . 72

5.3.2 Quantitative Density Measurements in the Cell Nucleus . . . . . . 79

5.3.3 DNA Aggregation and Density for a Cell Ensemble . . . . . . . . . 82

5.4 Summary and Conclusions . . . . . . . . . . . . . . . . . . 84

5.5 Supporting Information . . . . . . . . . . . . . . . . . . . 85

5.5.1 Visible Light Phase Contrast Microscopy . . . . . . . . . . . . . 85

5.5 .2 Comparison of Dark Fields . . . . . . . . . . . . . . 86

5.5 .3 Nucleoli Region of Interest . . . . . . . . . . . . . . . . . . . 87

5.5 .4 Supplementary Cell . . . . . . . . . . . . . . . . . . 88

5.5.5 Momentum Transfer Comparison . . . . . . . . . . . . . . . . . 91

$5.5 .6 \quad$ Cell Ensemble . . . . . . . . . . . . . . . . . . . . . . . . 91

6 Optical Fluorescence and X-ray Tomography Probe Cell Nuclei 93

6.1 Sample Preparation and Experimental Setups: Labeled Lyophilized and Hydrated Nuclei . . . . . . . . . . . . . . . . . . . . . . . 95

6.2 Results and Discussion: Labeled Lyophilized and Hydrated Nuclei . . . . 97

6.3 Sample Preparation and Experimental Setups: Tomography Recordings . 105

6.4 Results and Discussion: Tomography Recordings . . . . . . . . . . . . 105

$\begin{array}{lll}7 & \text { Summary and Conclusion } & 109\end{array}$

$\begin{array}{lr}\text { A Protocols } & 111\end{array}$

A.1 Grid Plunging. . . . . . . . . . . . . . . . . . . . . . . 111

A.2 Fluorescent Labeling of DNA . . . . . . . . . . . . . . . . . 113 
References 

List of Figures

3.1 Electromagnetic radiation schematic . . . . . . . . . . . . . 8

3.2 Jablonski diagram . . . . . . . . . . . . . . . . . . . . . . . . . . . . . . . 11

3.3 Epifluorescence microscope schematic . . . . . . . . . . . . . . . 13

3.4 Confocal microscope schematic . . . . . . . . . . . . . . . . 15

3.5 STED microscope schematic . . . . . . . . . . . . . . . . 17

3.6 Schematic representation of the Beer-Lambert law . . . . . . . . . . . 20

3.7 Mass attenuation coefficients corresponding to a protein . . . . . . . . . 21

3.8 Differential cross section schematic . . . . . . . . . . . . . . . . . . 22

3.9 Compton scattering schematic . . . . . . . . . . . . . . . . . 24

3.10 Thomson scattering schematic . . . . . . . . . . . . . 25

3.11 Geometric representation of Bragg's law . . . . . . . . . . . . . . . 27

3.12 Schematic representation of form and structure factors . . . . . . . . . . 29

3.13 Form factors of various objects . . . . . . . . . . . . 30

4.1 Preparation of hydrated cells on polypropylene foil . . . . . . . . . . . 35

4.2 Fluorescently labeled 3T3 fibroblasts nuclei . . . . . . . . . . . . . . 36

$4.3 \mathrm{Si}_{3} \mathrm{~N}_{4}$ window preparation overview . . . . . . . . . . . 37

4.4 Scanning SAXS setup at GINIX . . . . . . . . . . . . . . . . 42

4.5 Scanning SAXS data analysis overview . . . . . . . . . . . . . . 43

4.6 Porod constant and exponent map . . . . . . . . . . . . . . . . . . . . 44

4.7 Schematic representation of the Fresnel scaling theorem . . . . . . . . . . 46

4.8 Schematic representation of the error-reduction algorithm . . . . . . . . . 49

4.9 Holography setup at GINIX . . . . . . . . . . . . . . . 50

4.10 Data correction overview of a single hologram . . . . . . . . . . . 52

4.11 Reconstruction of holograms recorded at four defocused positions . . . . . 53

4.12 Radon transform projection at an arbitrary angle . . . . . . . . . . 55

4.13 Reconstruction overview using the Radon transform . . . . . . . . . . 56

4.14 Tomography setup at GINIX . . . . . . . . . . . . . . . . . 57

4.15 Overview of the tomography reconstruction process . . . . . . . . . 60

4.16 STED microscope optics box schematic . . . . . . . . . . . . . . 62

4.17 Schematic of the STED microscope implemented at the GINIX endstation 63

4.18 Lateral stage of the STED microscope implemented at the GINIX endstation 64

5.1 The GINIX scanning SAXS and holography experimental setups used during Run $57 \ldots \ldots \ldots \ldots$. . . . . . . . . . . . . . . . . . . . . . . . . . . 71

5.2 Parameter maps of a single cell . . . . . . . . . . . . . . . 73 
5.3 Various dark field images showing the granularity of a single cell . . . . . 75

5.4 Regions of interest and corresponding intensity profiles of a single cell . . 77

5.5 Histograms of variables belonging to 33 cells . . . . . . . . . . . . . 83

5.6 Visible light phase contrast images of lyophilized and chemically-fixed cells 86

5.7 Various dark field images with the same color scale showing the overall granularity of a cell . . . . . . . . . . . . . . . . . 87

5.8 Comparison between radial intensity profiles with and without the consideration of heterochromatin . . . . . . . . . . . . . 88

5.9 Parameter maps of a cell . . . . . . . . . . . . . . . . . . 90

5.10 Comparison between two 1D averaged azimuthally integrated power spectral densities . . . . . . . . . . . . . . . . . 91

6.1 Mounted $\mathrm{Si}_{3} \mathrm{~N}_{4}$ and polypropylene chamber . . . . . . . . . . . . 97

6.2 Confocal and STED images of hydrated and lyophilized fibroblasts . . . . 98

6.3 Comparison between lyophilized and hydrated confocal stacks . . . . . . . 100

6.4 Maximum and average intensity projections of confocal stacks . . . . . . . 102

6.5 Comparison of azimuthally averaged power spectral densities of fluorescent images . . . . . . . . . . . . . . . . . . . . 104

6.6 Reconstructed phase and projected mass density maps recorded at different angles . . . . . . . . . . . . . . . . . 106

6.7 3D images of a lyophilized nucleus . . . . . . . . . . . . . . 106

6.8 Reconstructed phase maps of a hydrated nucleus recorded at different angles 107

A.1 Overview of the Leica EM GP grid plunger . . . . . . . . . . . . . . . 112

A.2 Overview of the grid plunging process . . . . . . . . . . 113

B.1 Sample AW-PD06-C4 . . . . . . . . . . . . . . . . . 118

B.2 Sample AW-PD06-C5 . . . . . . . . . . . . . . . . . . . 119

B.3 Sample AW-PD06-C6 . . . . . . . . . . . . . . . . . . . . . . . . . . . . . . . . . . . . . . .

B.4 Sample AW-PD06-C7A . . . . . . . . . . . . . . . . . 121

B.5 Sample AW-PD06-C7B . . . . . . . . . . . . . . . . . 122

B.6 Sample AW-PD06-C8 . . . . . . . . . . . . . . . . . . . . 123

B.7 Sample AW-PD06-C10 . . . . . . . . . . . . . . . . . . . . . . . . 124

B.8 Sample AW-PD06-C11 . . . . . . . . . . . . . . . . . . . 125

B.9 Sample AW-PD06-C12 . . . . . . . . . . . . . . . . . . . 126

B.10 Sample AW-PD06-C13 . . . . . . . . . . . . . . . . . . . . 127

B.11 Sample AW-PD06-C14 . . . . . . . . . . . . . . . . . . 128

B.12 Sample AW-PD06-C15 . . . . . . . . . . . . . . . . . . . . . 129

B.13 Sample AW-PD06-C16 . . . . . . . . . . . . . . . . . . . . . . . . . . . . . . . . . . . . . . . . .

B.14 Sample AW-PD06-C17 . . . . . . . . . . . . . . . . . . 131

B.15 Sample AW-PD06-C18 . . . . . . . . . . . . . . . . . . . . . 132

B.16 Sample AW-PD06-C19 . . . . . . . . . . . . . . . . . . . . 133

B.17 Sample AW-PD06-C20 . . . . . . . . . . . . . . . . . . . . . . . . . . . . . . . . . . . . . . . . . . . . . .

B.18 Sample AW-PD06-C22 . . . . . . . . . . . . . . . . . . . . . . . . . . . . . . . . . . . . . . . . . .

B.19 Sample AW-PD06-C23 . . . . . . . . . . . . . . . . . . . . . . . . . . . . . . . . . . . . . . . .

B.20 Sample AW-PD06-C24 . . . . . . . . . . . . . . . . . 137

B.21 Sample AW-PD06-C26 . . . . . . . . . . . . . . . . . . . . . . . . . . . . . . . . . . . . . . . . .

B.22 Sample AW-PD06-C27 . . . . . . . . . . . . . . . . . 139 
B.23 Sample AW-PD06-C29 . . . . . . . . . . . . . . . . . . . . 140

B.24 Sample AW-PD06-C30 . . . . . . . . . . . . . . . . . . . . . . . . . . . . . . . . . . . . . . . . .

B.25 Sample AW-PD06-C31 . . . . . . . . . . . . . . . . . . . . . . . . . . . . . . . . . . . . . . . . . . . . . .

B.26 Sample AW-PD06-C35 . . . . . . . . . . . . . . . . . . . 143

B.27 Sample AW-PD06-C36 . . . . . . . . . . . . . . . . . . . . . . . . . . . . . . . . . . . . . . . . . .

B.28 Sample AW-PD06-C38 . . . . . . . . . . . . . . . . . . . . . . . . . . . . . . . . . . . . . . . . . . . . . . .

B.29 Sample AW-PD06-C40 . . . . . . . . . . . . . . . . . . . . . . . . . . . . . . . . . . . . . . . . . . .

B.30 Sample AW-PD06-C41 . . . . . . . . . . . . . . . . . 147

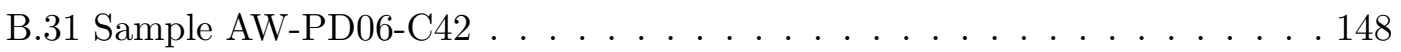

B.32 Sample AW-PD06-C43A . . . . . . . . . . . . . . . . . . . . . 149

B.33 Sample AW-PD06-C43B . . . . . . . . . . . . . . . . 150 

List of Tables

4.1 Scattering length values of X-rays for biological material . . . . . . . . . . 39

4.2 Holography experimental parameters of Run 57 . . . . . . . . . . . . . 51

4.3 Holographic tomography experimental parameters of Run 79 . . . . . . 58

5.1 Summary of the parameters probed by combining scanning SAXS and X-ray holography . . . . . . . . . . . . . . . . . . . 84

5.2 Median values of all parameters belonging to 33 cells . . . . . . . . . . . 92

6.1 Experimental parameters used to acquire confocal and STED images . . . 96

6.2 Confocal and STED resolution approximation . . . . . . . . . . . . 104 

Biological cells are the basic structural and functional units of all organisms. There are approximately 60 trillion eukaryotic cells within a human body alone [1]. A common feature among eukaryotic cells is the nucleus. The nucleus is a membrane-bound organelle which, in addition to directing the synthesis of proteins and ribosomes, houses deoxyribonucleic acid (DNA). DNA is considered the blueprint of all life as it contains the genetic information necessary to develop and operate living organisms. Genetic mutations can lead to a wide variety of diseases, ranging from Huntington's [2] and Osteoporosis [3], which are related to brain and bone deterioration, respectively, to Sickle-cell disease, which deprives organs of oxygen [4]. Indeed, the investigation of DNA allows for the identification of genes which trigger such diseases as well as the ability to diagnose them. Despite the critical importance of DNA, a complete understanding of its hierarchical structure is lacking due to its remarkable complexity. The hierarchical combination of different length scales fundamentally defines the function of a cell. The length scales of DNA structures range from $2 \mathrm{~nm}$ to $1 \mu \mathrm{m}$, corresponding to double helices and individual chromosomes, respectively [5-7]. On intermediate length scales, hierarchical structures of several hundreds of nanometers in size are formed. The exact details of the hierarchical architecture spanning these length scales is a current topic of debate $[5,8-11]$.

Understanding the hierarchical structure of DNA is facilitated by nanoscale imaging. X-rays serve as an ideal probe to image biological structures at this scale as their high penetration depth and short wavelength enables comparatively thick samples to be non-invasively imaged with, in principle, atomic resolution. It was X-ray diffraction, a method sensitive to the collective electron density of a sample, which enabled the 
double helix structure of DNA to be resolved [12-14]. This thesis uses an extension of $\mathrm{X}$-ray diffraction termed scanning X-ray diffraction. In this method, a sample is raster scanned in the focal spot of the X-rays, which is typically in the range of a few hundred nanometers. In doing so, diffraction patterns from each scan location are collected and their features are extracted and analyzed to create quantitative maps of the underlying structure of the sample with spatial resolution on the order of the beamsize. This method has long since been applied to a large array of both inorganic and organic materials, but has only recently emerged as a tool to investigate biological cells. A complementary approach to scanning X-ray diffraction is X-ray in-line holography [15], a lens-less imaging technique sensitive to the phase shift of the sample. This imaging modality can form quantitative images with a resolution below $100 \mathrm{~nm}$ and, in terms of radiation damage, is less invasive than scanning X-ray diffraction. In addition to X-rays, visible-light fluorescence enables biological structures to be imaged with, in principle, atomic resolution. Furthermore, this approach has the benefit that only specificallylabeled structures can be imaged. Thus, when correlating visible-light micrographs with $\mathrm{X}$-ray micrographs, information obtained from a specifically-labeled structure can be correlated with structural information obtained using X-rays.

The aim of this thesis is to combine X-ray microscopy with visible-light fluorescence microscopy, in both two and three dimensions, in order to quantitatively probe hierarchical and structural parameters of DNA within whole cell nuclei. As a model system, lyophilized NIH-3T3 fibroblasts are investigated. In chapter 2 an overview of the current state of the art of imaging DNA is given. The theoretical background of image formation and X-ray scattering is introduced in chapter 3, and experimental methods used to collect and analyze data are introduced in chapter 4 . Chapter 5 details the results of combining scanning small-angle X-ray scattering and in-line holography to probe the 2D hierarchical structure of DNA. Chapter 6 discusses the results of combining X-ray tomography and visible-light fluorescence to probe the 3D structural parameters of DNA. Chapter 7 closes this work with a brief summary of the results and an outlook for future experiments and challenges. 
Within eukaryotic cells the nucleus plays multiple critical roles, ranging from storing genetic information to coordinating protein synthesis and cell division. The biological and architectural understanding of the nucleus has continuously evolved over the past 150 years. In 1871, while developing methods to isolate nuclei from white blood cells, Swiss physician Friedrich Miesche discovered a phosphorus-rich acid which he termed "nuclein" [7, 16]. Today, nuclein is known as deoxyribonucleic acid (DNA). Around 1880, German biologist Walther Flemming investigated the cell division process and found a structure which strongly absorbed basophilic dyes [17]. He named this structure chromatin. Chromatin is primarily composed of DNA and histones, a protein that interacts with DNA and assists in compacting it. Histones were discovered in 1884 by German biochemist Albrecht Kossel [18]. The remainder of the $19^{\text {th }}$ century and first half of the $20^{\text {th }}$ century saw great strides in the emerging field of genetics, but was largely devoid in advancing the understanding of the structure of DNA. It was not until 1944 when new light was shed on DNA by Avery, MacLeod and McCarty [19], who experimentally showed that DNA is responsible for bacteria transferring genetic information, thus verifying the "transforming principle" proposed by British bacteriologist Frederick Griffith in 1928 [20]. A few years later, in 1951, American chemist Linus Pauling determined the $\alpha$-helix structure of polypeptides that makes up the secondary structure of many proteins [21] using X-ray diffraction. Pauling is considered one of the most influential scientists of the $20^{\text {th }}$ century and it is his work on chemical bonds [21, 22] that enabled Rosalind Franklin, Maurice Wilkins, James Watson and Francis Crick to resolve the double helix structure of DNA using X-ray diffraction in 1953 [12-14]. The success of using X-ray diffraction to probe the structure of DNA and proteins stimulated a number of laboratories to use X-ray diffraction and small-angle X-ray scattering to elucidate 
chromatin structure [7, 23-25]. Electron microscopy was subsequently employed to complement X-ray data. In 1970, Davies and co-workers used electron microscopy to image thin slices of condensed chromatin and found supercoils to be highly aligned in parallel arrays beneath the nuclear envelope [7, 26]. Furthermore, in the early 1970s, electron microscopy enabled the visualization of the nucleosome, the fundamental repeating subunit of chromatin [27-29]. In 1984, the nucleosome crystal structure was determined at a resolution of 7.0 $\AA$ using X-ray diffraction by Aaron Klug and co-workers [30].

Today, imaging nuclear material within biological cells uses three primary probes: $\mathrm{X}$ rays, visible light fluorescence and electrons. By combining time-lapse bright-field microscopy with scanning small-angle X-ray scattering, Hémonnot et al. [31] followed the cell cycle of whole lyophilized NIH-3T3 fibroblasts in a temporal manner and related the observed structural changes to specific stages in the cell cycle. The aggregation and (de)-compaction of chromatin was probed and pronounced compaction was associated with the early stages of the cell cycle. Jiang et al. [32] used 3D X-ray diffraction to quantitatively image whole Schizosaccharomyces pombe yeast spore cells. The authors determined the volume of the nucleus to be $0.28 \mu^{3}$, with the nucleolus to have a mass density of approximately $1.6 \mathrm{~g} \cdot \mathrm{cm}^{-3}$. Giewekemeyer et al. [33] used X-ray ptychography to probe unsliced and unstained lyophilized cells of the bacterium Deinococcus radiodurans. The authors quantified the mass density projected along the optical axis and attributed particularly dense regions to be compacted plasmid DNA, with a typical mass per area of $0.17 \mathrm{mg} \cdot \mathrm{cm}^{-2}$. Motivated by this success, Bartels et al. [34] also imaged Deinococcus radiodurans only in a hydrated and living state using X-ray in-line holography. The dense regions attributed to plasmid DNA observed in the lyophilized state were also observed in the hydrated and living state. Comparable projected mass densities values were observed. To resolve local mass density, rather than the projected mass density, the 2D approaches of ptychography and in-line holography can be extended to 3D. Bartels et al. [35] combined in-line holography with tomography, so-called "holotomography" [36], to image unstained and unsliced gram-positive lyophilized Deinococcus radiodurans. Characteristic dense regions were observed which the authors attributed to be rich in DNA. These regions had densities in the range of 1.2 to $1.65 \mathrm{~g} \cdot \mathrm{cm}^{-3}$. Similarly, Wilke et al. [37] combined tomography with ptychography to image lyophilized Deinococcus radiodurans. The 3D mass density distribution was resolved in real space with $50 \mathrm{~nm}$ resolution. Round, globular regions approximately isotropic in shape were observed and attributed to be DNA-dense regions with a mass density of $1.6 \mathrm{~g} \cdot \mathrm{cm}^{-3}$, thus being in very good agreement with Bartels et al. [35]. In the same work, Wilke et al. [37] combined 2D ptychography data with scanning X-ray diffraction data. The ptychography data allowed for a fast and robust visualization of the biological cells without the invasive need of staining. Furthermore, the X-ray diffraction data in Fourier space 
complemented the Fourier information obtained by ptychography. Diaz et al. [38] also combined ptychography with tomography to map the 3D electron density of the green alga Chlamydomonas reinhardtii embedded in resin. With an isotropic resolution of $180 \mathrm{~nm}$, the authors were able to identify several organelles, including the nucleus and nucleolus. The authors quantified the average 3D electron density of nucleus to be $450 \mathrm{e}^{-} \cdot \mathrm{nm}^{-3}$.

Transitioning from solely X-ray-based techniques, Song et al. [39] combined 3D coherent X-ray diffraction microscopy with $2 \mathrm{D}$ optical fluorescent microscopy to image cell nuclei of mouse fibroblasts. The diffraction imaging enabled the explicit 3D visualization of nuclear substructures at several ten of nanometer resolution. The authors determined the average electron density of the nucleus and nucleolus to be $440 \mathrm{e}^{-} \cdot \mathrm{nm}^{-3}$ and $620 \mathrm{e}^{-} \cdot \mathrm{nm}^{-3}$, respectively. The authors attributed specific regions within the nucleus to be heterochromatin and associated them with bright spots observed in the fluorescent data. Using only fluorescence microscopy, Spahn et al. [40] incorporated a method to provide a constant exchange of intact fluorophores with 3D STED microscopy to obtain, in principle, unlimited acquisition times while circumventing the effects associated with photobleaching. The authors demonstrated this method on Escherichia coli and chemically-fixed HeLa cells. In E. coli, the 3D radial positioning of DNA in proximity to the bacterial plasma membrane was visualized. In HeLa cells, mitotic chromosomes were observed to have a grained chromosome structure and small DNA loops. These features have also be observed using electron microscopy by Marsden et al. [41]. To visualize the in situ 3D ultrastructure of chromatin in human mitotic chromosomes, Ou et al. [42] introduced "ChromEMT", a method which combines a DNA-specific fluorescent label with electron microscopy tomography. Rather than the classically considered hierarchically folded assembly, the authors concluded that chromatin can best be described as a disordered and flexible granular chain. Motivated by the work of Ou et al. [42], Li et al. [43] introduced a method to quantitatively image the 3D distribution of chromatin in A549 lung cancer cells. The method, termed "ChromSTEM", exploits high angle annular dark field imaging and tomography in scanning transmission electron microscopy in combination with a DNA-specific fluorescent label compatible with electron microscopy. The authors observed chromatin to be arranged in spatially well-defined packing domains which exhibit a fractal internal structure. Concentrated regions of DNA were found to have an average mass density of approximately $2.0 \mathrm{~g} \cdot \mathrm{cm}^{-3}$. The authors observed heterochromatin to be, on average, 2.4 times more dense than euchromatin.

In comparison to the aforementioned examples, within the scope of this thesis scanning small-angle X-ray scattering and X-ray in-line holography are used in a complementary manner to image whole cell nuclei. Furthermore, whole cell nuclei are imaged using 
a combination of holotomography and visible-light fluorescence. The following chapter discusses the theoretical background necessary to help the reader interpret the results. 


\section{Light-Matter Interactions}

Within the scope of this thesis visible light microscopy techniques are used to visualize whole cell nuclei of 3T3 fibroblasts. In the first part of this chapter, an introduction to the principles of visible light microscopy are given.

It is unclear who developed the first microscope, but Dutch scientist Antoni van Leeuwenhoek is often credited with designing the first single lens microscope in the middle of the $17^{\text {th }}$ century. At a time when people generally didn't consider that life may be composed of entities smaller than what was observed with the naked eye, van Leeuwenhoek was remarkably observing bacteria [44]. Later in the $17^{\text {th }}$ century, Englishman Robert Hooke developed a compound microscope which incorporated a series of lenses. As centuries progressed, enormous leaps in microscopy advancements took place and many different techniques were developed.

\subsection{Optical Image Formation}

The electromagnetic radiation (EMR) spectrum is composed to two transverse oscillating fields: the magnetic $(\vec{B})$ and electric $(\vec{E})$, as shown in Figure 3.1. The fields are in phase and are characterized by the wavelength $\lambda$, defined as the distance between two consecutive maxima. Visible light is the portion of the EMR visible to the human eye and ranges from violet to red with corresponding wavelengths of approximately 400 to $700 \mathrm{~nm}$. 


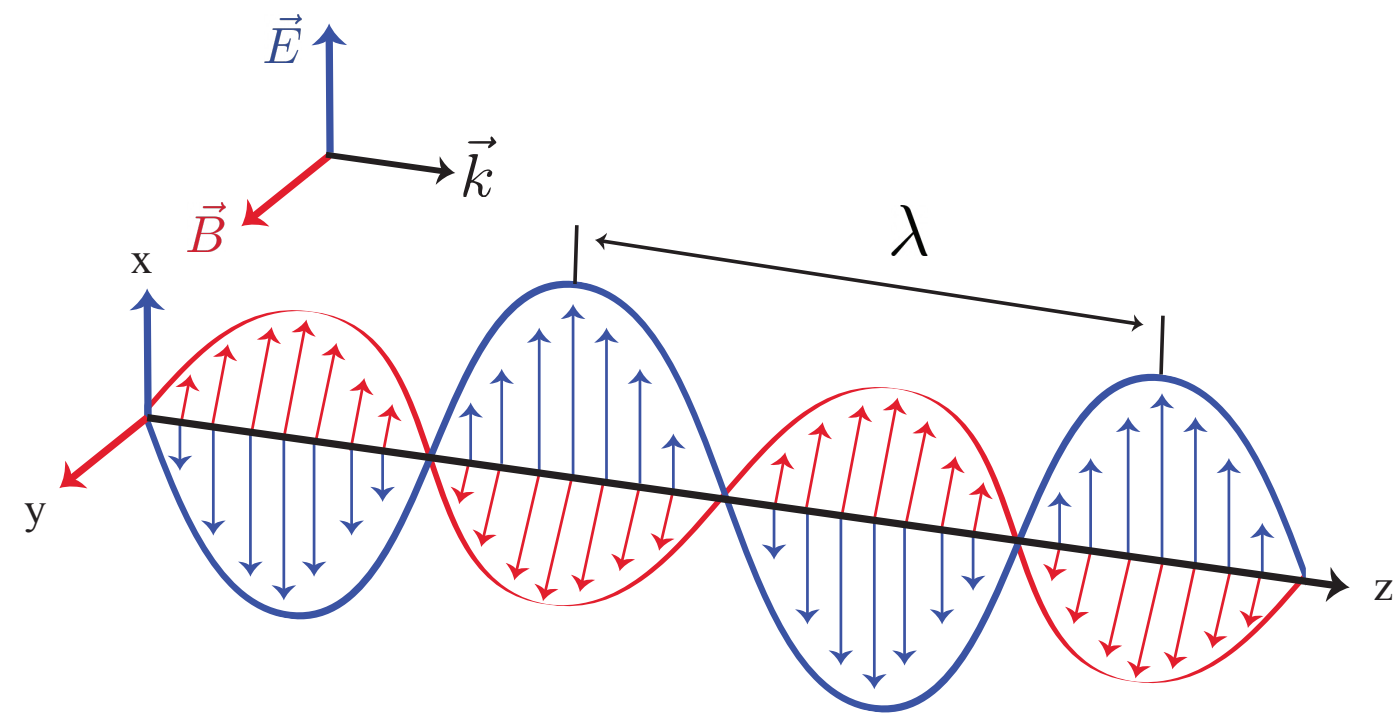

FIgURE 3.1: Schematic of the oscillating EMR field. The magnetic (red) and electric (blue) fields propagate at $90^{\circ}$ to one another along the wavevector $\vec{k}$.

In a microscope, light emitted from a point-like source is used to form an image with a lens, the resulting image will not be a point but rather a three-dimensional diffraction pattern, termed an Airy pattern, resembling a blurred intensity distribution centered around the geometrical focal point at $\vec{r}=\left\langle x_{0}, y_{0}, z_{0}\right\rangle$. The blurring effect is a direct consequence of the focusing of light that has been diffracted at the circular aperture of the lens [45]. The Airy pattern is symmetrically periodic along the optical axis of the microscope, and radially symmetric across the image plane. A cross section of the Airy pattern in the focal plane results in a bright disk, termed an Airy disk, in the center with alternating bright and dark higher-order diffraction rings which decrease in intensity as the distance from the Airy disk increases.

The electric field distribution at $\vec{r}=\langle x, y, z\rangle$ is termed the amplitude point spread function $h_{A}(\vec{r})$ and can be calculated via 3D scalar diffraction theory [46]:

$$
h_{A}(\vec{r})=C \int_{0}^{\alpha} \sin (\theta) \sqrt{\cos (\theta)} \cdot J_{0}\left(k \sin (\theta) \sqrt{x^{2}+y^{2}}\right) \cdot \exp [i k z \cos (\theta)] d \theta,
$$

where $C$ is a normalization constant and $\theta$ is the polar aperture angle that ranges from 0 to to the maximum opening angle $\alpha$. The term $J_{0}$ is a zero-order Bessel function of the first kind. The wave number $k$ is dependent on the refractive index $n$ of the medium (e.g., air, water or oil) and the wavelength of light in vacuum $\lambda_{0}: k=2 \pi n \cdot \lambda_{0}^{-1}$. As most detectors measure the intensity and not electric field distribution, it is useful to calculate the intensity point spread function (PSF) to describe the imaging process. It 
is given by the absolute square of the amplitude point spread function:

$$
\mathrm{PSF}=\left|h_{A}(\vec{r})\right|^{2}
$$

In general, the PSF of an imaging system describes the Airy pattern of a point emitter. Consequently, the image $I(\vec{r})$ of an object $O(\vec{r})$ is given by the convolution of the object and PSF:

$$
I(\vec{r})=O(\vec{r}) * \mathrm{PSF} .
$$

As a result, the imaging process will inherently lead to a blurred image. To minimize this effect and obtain a sharp image, the PSF needs to be minimized or deconvolved. According to the Rayleigh criterion, two objects are spatially resolvable if the first diffraction minimum, corresponding to one object, coincides with the first diffraction maximum of the second object [47].

To measure the lateral resolution of an image, the radius $r_{x, y}$ of the Airy disk is typically used:

$$
r_{x, y}=\frac{.61 \lambda}{\mathrm{NA}}
$$

where NA is the numerical aperture of the objective and is defined as: $\mathrm{NA}=n \sin (\theta)$, where $\theta$ is the semi-aperture angle of the lens and $n$ is the index of refraction. Analogous to the lateral resolution is the axial resolution, which is defined by the distance between the maximum and first minimum in the axial direction of the diffraction pattern. It is expressed as:

$$
r_{z}=\frac{2 \cdot n \lambda}{\mathrm{NA}^{2}}
$$

Often, the central part of the Airy pattern is approximated by a Gaussian function. In this case, both the axial and lateral resolutions are expressed by its full-width at half maximum:

$$
\begin{aligned}
\tilde{r}_{x, y} & =\frac{.51 \lambda}{\mathrm{NA}} \\
\tilde{r}_{z} & =\frac{1.77 \cdot n \lambda}{\mathrm{NA}^{2}}
\end{aligned}
$$

\subsection{Visible-Light Fluorescence Microscopy}

One of the simplest forms of microscopy is bright field. Here, a sample is fully illuminated by light from an incandescent source and contrast is formed by the absorption of light in dense regions of the sample. The transmitted light is then used to form an image. However, for weakly absorbing samples such as biological cells, the contrast is very poor. 
To overcome this limitation, fluorescence light is incorporated to image specificallylabeled structures.

\subsubsection{Basic Principles}

A molecule which can absorb light of wavelength $\lambda_{1}$ and emit light with a wavelength of $\lambda_{2}$ is known as a fluorophore. When a fluorophore absorbs a photon with energy $E$, the outer-most electron absorbs the energy. If the energy is equal to or greater than the energy gap between the ground state $S_{0}$ and first excited state $S_{1}$, the electron will be become excited and transition from $\mathrm{S}_{0}$ to $\mathrm{S}_{1}$. The absorption process occurs on the order of a femtosecond and, at this timescale, the structure of the fluorophore doesn't have time to change; this is known as the Franck-Condon principle. The absorbance of a fluorophore quantifies how much of the incident light will be absorbed by it, rather than, for example, how much will be reflected or refracted. The absorbance $A$ is described by the Beer-Lambert law [48]:

$$
A=\log \left(\frac{I_{0}}{I}\right) \approx \lambda d c \epsilon,
$$

where $I_{0}$ and $\lambda$ are the intensity and wavelength of the incident light, $I$ is the intensity of the transmitted light after travelling some distance $d$ and $c$ is the molar concentration of the absorbing fluorophore. If a single photon is absorbed, the variable $\epsilon$ is the molar extinction coefficient and is related to the one-photon cross section of the absorbing fluorophore. There are three main components which contribute to the absorption process:

1. Electronic: Corresponds to the electronic gap transition and is the only component which allows for the electron to transition from one state to another e.g., from $\mathrm{S}_{0}$ to $\mathrm{S}_{1}$. Light within the UV to visible regime $(\lambda \sim 200-700 \mathrm{~nm})$ is usually required to fill this energy gap.

2. Vibrational: Within a given state $\left(S_{n}\right)$ there exist multiple vibrational levels. To change from one level to another, light in the IR regime $(\lambda \sim 700 \mathrm{~nm}-1 \mathrm{~mm})$ is usually required.

3. Rotational: Within each vibrational level there exist multiple rotational states. Changing from one state to another usually requires energy in the microwave regime $(\lambda \sim 1-1000 \mathrm{~mm})$.

Thermal energy alone usually is not sufficient to populate the vibrational energy state and excite the electron from the ground state $S_{0}$ to $S_{1}$; a photon with adequate energy $E$ is typically required. Alternatively two photons, both of which have energy equal to 
$E / 2$, can excite the fluorophore to $\mathrm{S}_{1}$ via the two-photon excitation [49] process. The excitation and de-excitation process can be schematically explained using a Jablonski diagram [50], as shown in Figure 3.2. Within this figure, straight lines refer to nonradiative transitions and oscillating lines refer to radiative transitions. Unless the energy absorbed by the photon is exactly equal to that of the electronic gap, which would result in the electron being excited to the lowest energy level in $\mathrm{S}_{1}$, the electron is excited to one of the higher vibrational levels in $\mathrm{S}_{1}$, as depicted by the blue arrow in Figure 3.2. From the higher vibrational level, the electron will de-excite in a non-radiative manner to the lowest vibrational level via internal conversion (green arrow).

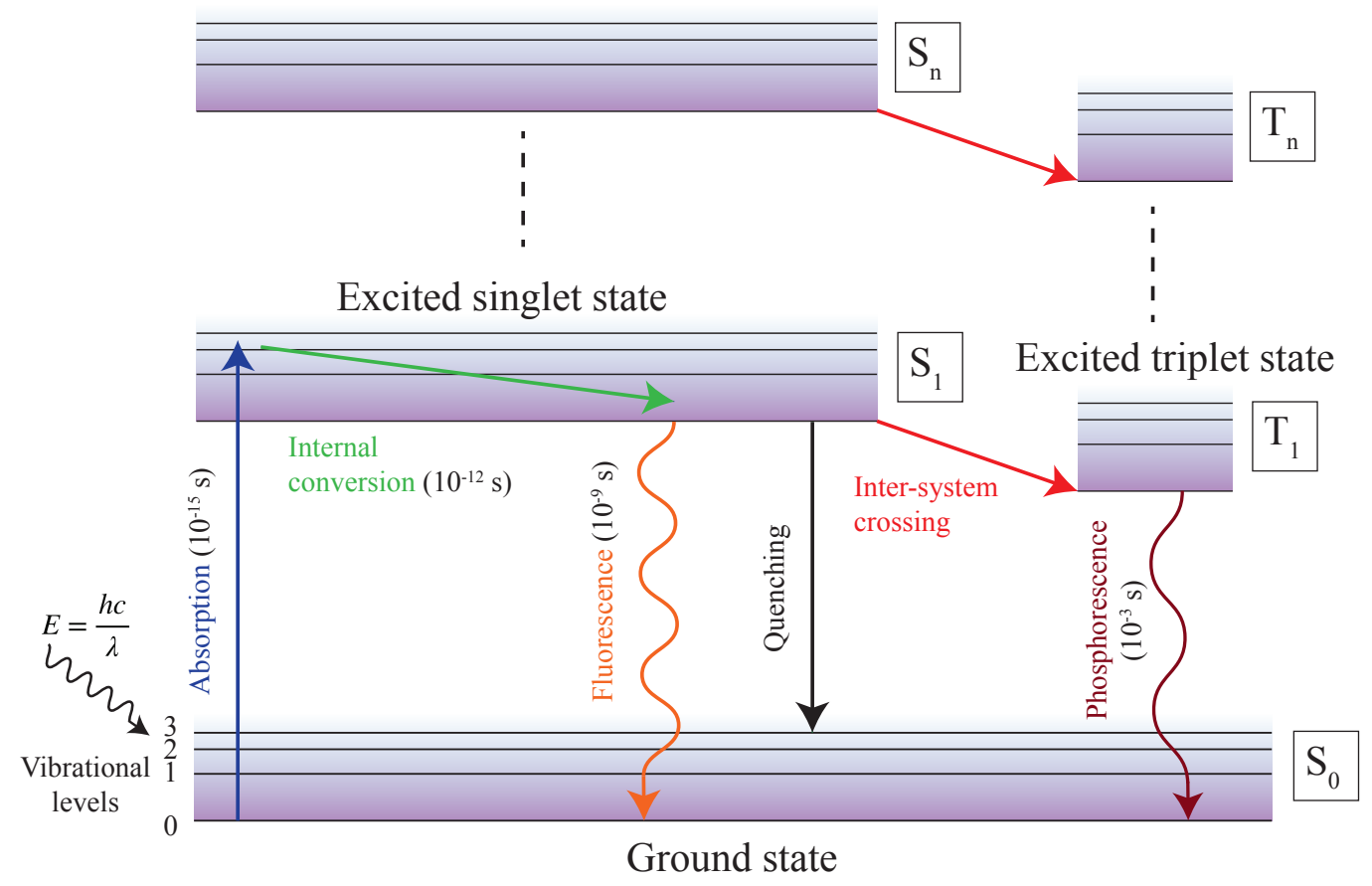

Figure 3.2: Schematic of a Jablonski diagram, showing the pathways in which an excited molecule can return to the ground state $S_{0}$. Straight and oscillating lines represent non-radiative and radiative paths, respectively. An incoming photon with energy $E=h c / \lambda$, which is equal to at least the energy difference between the first excited state $S_{1}$ and the ground state, is absorbed by the molecule (blue arrow). The excited molecule relaxes to the lowest energy level of the excited state via internal conversion (green arrow). The molecule can then return to the ground state via fluorescence (orange oscillation) or in a non-radiative manner, such as quenching (black arrow). Alternatively, the molecule can transition to the triplet state via inter-system crossing (red arrow) and decay to the ground state via phosphorescence (dark-red oscillation).

Once at the lowest vibrational level in the $\mathrm{S}_{1}$ state, the electron can return to the ground state $\mathrm{S}_{0}$ by fluorescence (orange oscillation), which has a lifetime $\tau_{\text {fluo }}$ on the order of a few nanoseconds. Due to the energy dissipation associated with inter-system crossing, fluorescence usually occurs at a lower energy, and therefore has a larger wavelength, when compared to the incident photon. If fluorescence doesn't occur, the electron can 
spontaneously decay to $\mathrm{S}_{0}$ through a non-radiative manner quenching process (black arrow) e.g., stimulated emission (see Section 3.2.4). Alternatively, the electron can dissipate energy and transition to the excited triplet state $\mathrm{T}_{1}$ via a non-radiative process known as inter-system cooling (red straight line). From the excited triplet state, the electron can radiatively decay to the ground state; this process is known as phosphorescence (dark-red oscillating line) and has a lifetime on the order of milliseconds to hours. Due to inter-system cooling, phosphorescence has a longer wavelength than fluorescence light. The difference in wavelength between the absorbed photon and emitted photon was first describe in 1852 by George Gabriel Stokes [51] and is therefore known as the Stokes shift. It allows for the distinction between excited light and emitted light [52]. In the following sections microscopy techniques which exploit the principles of fluorescence are discussed.

\subsubsection{Epifluorescence Microscopy}

A basic fluorescence microscope is a conventional compound microscope equipped with a high intensity light source with a large broadband spectrum, usually within the visible to ultra violet regime. The light source is typically a xenon arc lamp or a mercury vapor lamp. However, high-power lasers or light emitting diodes, which have a comparatively narrow broadband, are also used. An epifluorescence microscope is a diffraction-limited fluorescence microscope in which the hardware is configured to allow for both the excitation and emitted light to travel through the objective. In doing so, the objective can simultaneously condense the illuminated light and collect the fluorescent light.

The excitation light from the source initially passes through a filter which transmits light within a narrow wavelength range, corresponding to the excitation wavelength of the fluorophores within the sample. The transmitted light is then reflected by the dichroic mirror and is focused onto the sample by the objective. A schematic of the excitation pathway is shown in green in Figure 3.3. The incident light excites the fluorophores within the sample, which in turn emit fluorescent light. The fluorescent light is collected by the objective is transmitted by the dichroic mirror. It can then passes through an emission filter, which transmits the emitted light and blocks any residual excitation light, before impinging on the detector. A schematic of the emission pathway is shown in red in Figure 3.3. 


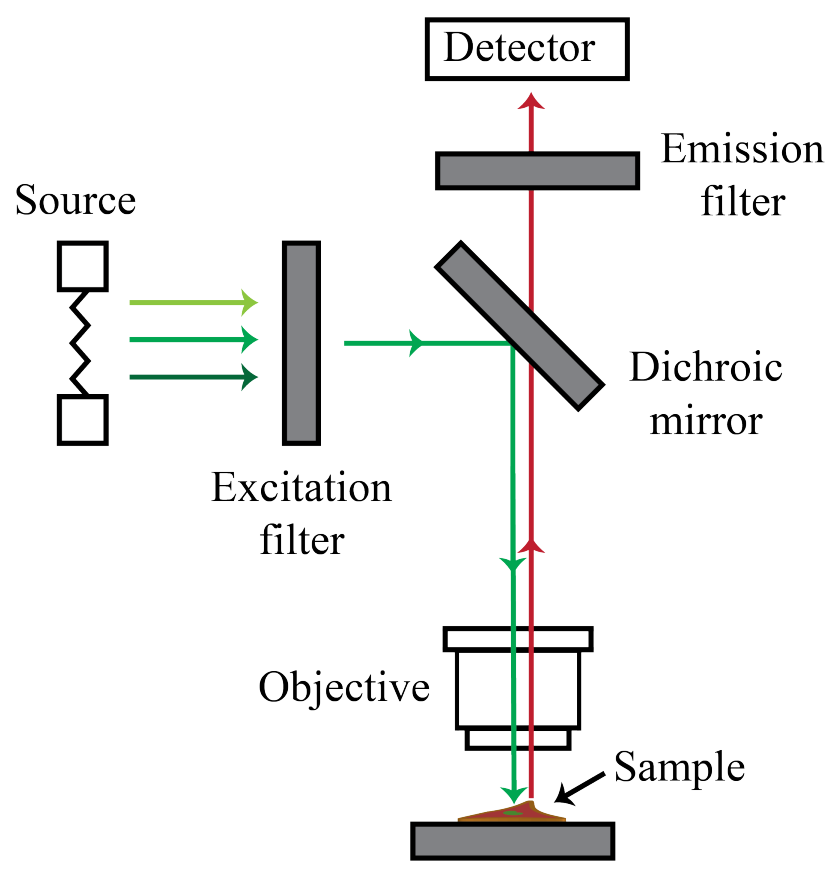

FIGURE 3.3: Schematic overview of an epifluorescence microscope. The excitation light (green) emitted from the source passes through an excitation filter. The transmitted light is reflected by the dichroic mirror, is focused onto the sample by the objective and excites fluorophores within the sample. The excitation light (red) is transmitted by the dichroic mirror and is filtered by the emission filter prior to being collected by the detector.

Epifluorescence fully illuminates the sample, thus rendering all excited fluorophores within the irradiated volume to fluoresce. Consequently, the detected signal is attributed to all excited fluorophores within the irradiated volume. Thus no information regarding axial resolution is obtained. As discussed in the following text, advancements in fluorescent microscopy techniques enable the visualization of $3 \mathrm{D}$ volumes with increased lateral and axial resolution.

\subsubsection{Confocal Microscopy}

In contrast to epifluorescence microscopy where the sample is fully illuminated, confocal microscopy restricts the illumination to a diffraction-limited spot, resulting in a spatial intensity distribution around the focal point in the sample plane. This intensity distribu-

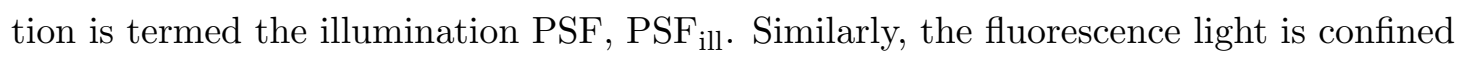
by placing a pinhole in the conjugate focal plane at the position of the geometrical focal point, thus only photons emitted by the fluorophore in the focal plane will be collected. The resulting image in the detection plane is the detection PSF, PSF det. The effective PSF of a confocal microscope, $\mathrm{PSF}_{\mathrm{conf}}^{\mathrm{eff}}$, is proportional to the illumination and detection 
PSF:

$$
P S F_{\mathrm{conf}}^{\mathrm{eff}} \propto P S F_{\mathrm{ill}} \cdot P S F_{\mathrm{det}} .
$$

Analogous to equation 3.6, the lateral resolution of a confocal microscope is calculated using the FWHM of eq. 3.9 and is given by $[53,54]$ :

$$
\tilde{r}_{x, y, \mathrm{conf}}=\frac{.44 \lambda}{\mathrm{NA}} \text {. }
$$

Similarly, the axial resolution is expressed as:

$$
\tilde{r}_{z, \mathrm{conf}}=\frac{1.4 \cdot n \lambda}{\mathrm{NA}^{2}} .
$$

The combined effect of restricting both the illuminating and fluorescent light enables the imaging of structures within a specimen, e.g., biological tissue or cell body, via the process known as optical sectioning. The excitation light is focused to a diffractionlimited spot within the sample and is absorbed by the fluorophores within the sample, resulting in the emission of fluorescent light. The fluorescent light passes through the objective and is subsequently focused onto the detector. The presence of a pinhole ensures that only focused light is detected and any out-of-focus light is blocked. A schematic of the illuminating and excited light paths of a confocal microscope are shown in Figure 3.4a and b, respectively. As the schematic depicts, the detected photons originate from a single point within the illumination profile. To acquire an extended $2 \mathrm{D}$ image, the sample is typically raster scanned through the illuminating profile in the $x-y$ plane. A confocal stack of images can be acquired by adjusting the focal plane in the $z$ direction and subsequently raster scanning the sample in the $x-y$ plane. In doing so, the images can stacked together and used to render a 3D image. 


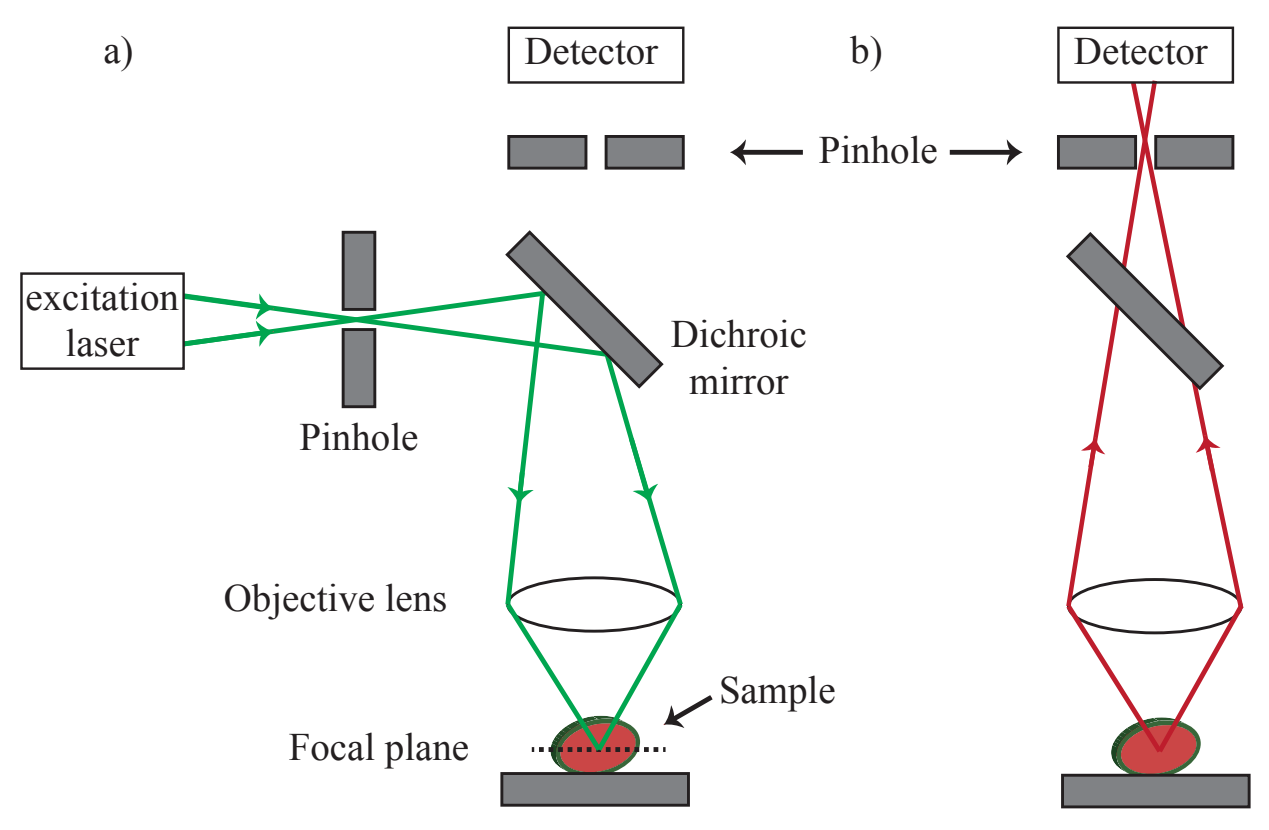

FiguRe 3.4: Schematic overview of a confocal microscope. a) The excitation light (green) is reflected by the dichroic mirror and focused to a point within the sample by the objective. b) The fluorescent light (red) light is transmitted by the dichroic mirror and any defocused light is blocked by the pinhole prior to being detected.

For both confocal and epifluorescence microscopy, the acquired images are diffractionlimited. For visible light this equate to a resolution of roughly $200 \mathrm{~nm}$ in the $x-y$ plane and $600 \mathrm{~nm}$ in the $z$ plane. In the 1990's the diffraction limit of visible light was broken using STED microscopy [55].

\subsubsection{Stimulated Emission Depletion Microscopy}

Stimulated-emission depletion (STED) microscopy was first proposed by Stefan Hell and Jan Wichmann in 1994 [55] as a tool create a sub-diffraction sized PSF. It was first experimentally demonstrated in 1999 by Thomas Klar and Hell [56] when they achieved a resolution of $106 \mathrm{~nm}$ imaging nanocrystals. STED microscopy has been used to image a variety of systems. For example, nuclear pore complexes [57] and a single fluorophore [58] has been imaged with lateral resolutions of $20 \mathrm{~nm}$ and $16 \mathrm{~nm}$, respectively. Nitrogenvacancy centers within crystals has been imaged with a resolution of $5.8 \mathrm{~nm}$ [59]. Of particular interest to the work presented in this thesis, STED has been used to investigate biological material within cell nuclei. Towards this end, STED microscopy has enabled the identification and detection of structural changes in chromatin domains with length scales of 40 to $70 \mathrm{~nm}$ [60], as well as the identification of individual chromatin proteins [61]. 
In STED microscopy, excited fluorophores in a diffraction-limited focus spot are deprived of their ability to fluoresce due to the exposure of a second, diffraction-limited beam which induces stimulated-emission. As a result, the effective PSF is below the diffraction limit. Stimulated emission is the process in which an excited fluorophore non-radiatively decays to the ground state by interacting with a photon whose energy is equal to the energy difference between the exited state and ground state. As the fluorescence lifetime $\tau_{\text {fluo }}$ is on the order of a few nanoseconds, a pulsed depletion laser with a duration of $\sim 0.1 \mathrm{~ns}$ is typically used. A schematic of a STED microscope is shown in Figure 3.5a; the excitation and STED laser beams are shown in green and red, respectively. The fluorescent light (yellow) is transmitted through the dichroic mirrors prior to being detected by, e.g., an avalanche photodiode.

Typically, the STED intensity $I_{\mathrm{STED}}$ resembles a doughnut-shaped intensity distribution with zero intensity $I_{\mathrm{STED}}(\vec{r}=0)=0$ at the center [62], as schematically shown in the center of Figure 3.5b. The zero intensity is not restricted to a doughnut-shaped distribution but can also be formed by multiple grooves [58] or planes [63]. In the doughnut configuration, $I_{\mathrm{STED}}$ is superimposed on the excitation PSF (Figure 3.5b, left) to render any fluorophores within the overlapping regions to decay to higher vibrational levels within the ground state $\mathrm{S}_{0}$, where they have a lifetime $\tau_{\text {vibr }}$ of a few picoseconds before transitioning to the lowest vibrational level. Consequently, only fluorophores within the central part of $I_{\text {STED }}$ remain excited (Figure $3.5 \mathrm{~b}$, right). 


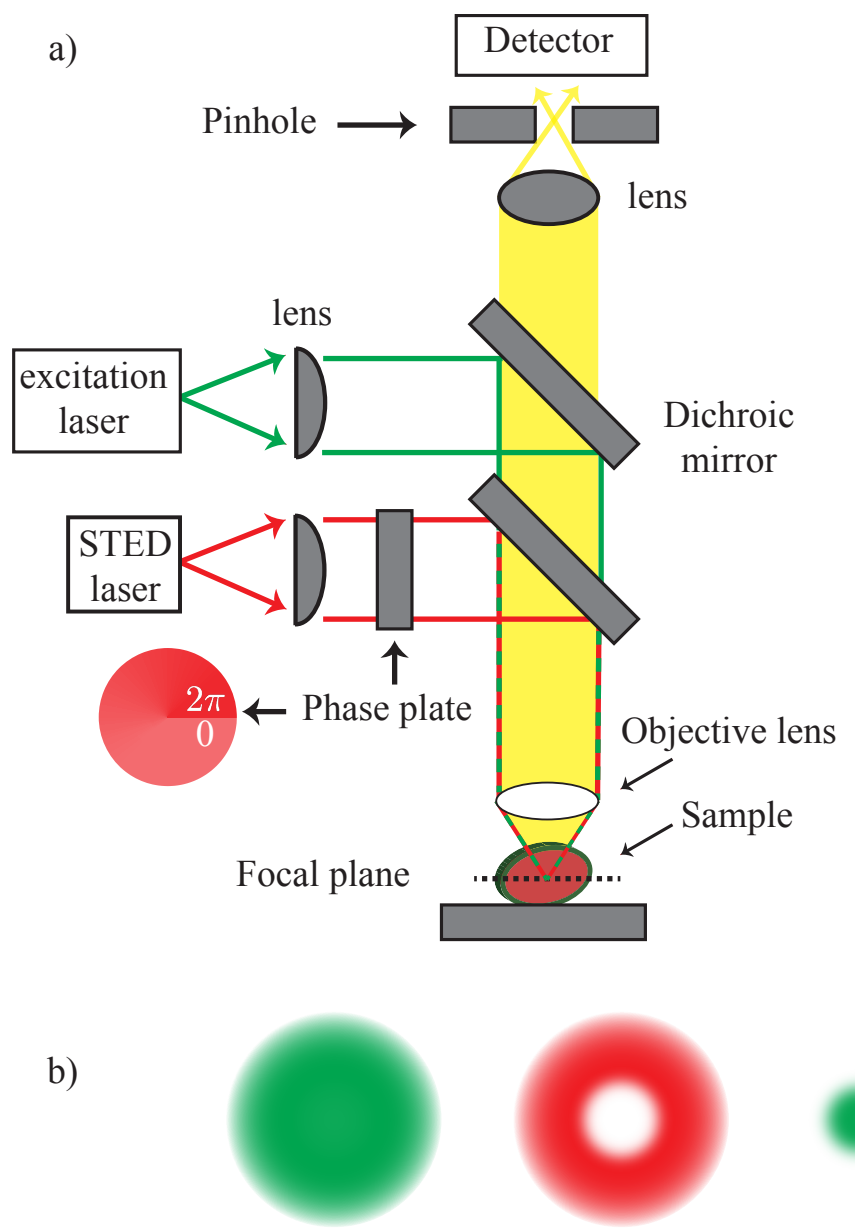

Figure 3.5: Schematic of a STED microscope. a) The excitation light (green) is reflected by the dichroic mirror and is focused onto the sample. The STED beam (red) is transmitted through a phase plate to render an intensity profile resembling a doughnut. The fluorescent light (yellow) passes through a pinhole prior to being detected. b) The diffraction-limited excitation PSF (left) is superimposed with the diffraction-limited STED PSF (center) to render the excited fluorophores within the overlapping regions to decay to the ground state via stimulated emission. The effective STED PSF (right) is below the diffraction limit.

The transition rate of stimulated emission is given by:

$$
\kappa_{\mathrm{STED}}(\vec{r})=\sigma \cdot I_{\mathrm{STED}}(\vec{r})
$$

where $\sigma$ is the molecular cross section for stimulated emission and has typical values of $10^{-16}$ to $10^{-17} \mathrm{~cm}^{2}$ [55]. To ensure the fluorescence signal is confined to the central de-excitation region of the STED beam, and that the fluorophores are effectively deexcited, the transition rate from $\mathrm{S}_{1} \longrightarrow \mathrm{S}_{0}$ via stimulated emission, $\kappa_{\mathrm{STED}}(\vec{r})$, must be greater than the spontaneous decay rate, $\kappa_{\text {fluo }}(\vec{r})=\left(\tau_{\text {fluo }}\right)^{-1}$, due to, e.g., fluorescence. 
Therefore, $I_{\mathrm{STED}}$ must be greater than the saturation intensity $I_{\text {sat }}$ of the fluorophore:

$$
I_{\mathrm{STED}}(\vec{r}) \gg \frac{1}{\sigma \tau_{\text {fluo }}} \equiv I_{\text {sat }}
$$

The term $I_{\text {sat }}$ is the effective saturation intensity and is defined as the intensity at which the probability of fluorescence emission is reduced by a factor of two. [64]. It typically has values of $10^{6} \mathrm{~W} \cdot \mathrm{cm}^{-2}$ [62]. The fraction of fluorescence still detected in the presence of the STED beam is described by the suppression factor $\eta(\vec{r})$ and is expressed as the exponential $[64,65]$ :

$$
\eta(\vec{r})=\exp \left[-\ln (2) \cdot I_{\mathrm{STED}}(\vec{r}) / I_{\mathrm{sat}}\right] .
$$

To determine the resulting spot size, and thus resolution, the effective STED PSF, $\mathrm{PSF}_{\mathrm{STED}}^{\mathrm{eff}}$, is calculated. To this end, the suppression factor is multiplied by the confocal PSF:

$$
P S F_{\mathrm{STED}}^{\mathrm{eff}}=P S F_{\mathrm{conf}}^{\mathrm{eff}} \cdot \eta(\vec{r}) .
$$

The lateral resolution is given by the FWHM of effective STED PSF [62]:

$$
\tilde{r}_{x, y, \mathrm{STED}}=\frac{\lambda}{2 n \sin (\alpha) \cdot \sqrt{1+I_{\max } / I_{\mathrm{sat}}}},
$$

where $I_{\max }$ is the maximum STED intensity confining the central de-excitation region. In theory, $I_{\max } / I_{\text {sat }}$ can approach infinity, thus enabling STED micrographs to obtain infinite resolution. In practice, STED micrographs generally yield resolutions larger than $20 \mathrm{~nm}$ $[55,62]$. As the intensity along the axial direction is zero, the use of a doughnut-shaped depletion pattern confines the excited volume only in the lateral directions. To achieve a resolution increase in the axial direction, a STED intensity distribution must be applied both above and below the focal plane. This can be achieved by, e.g., using a circular phase plate to apply a phase retardation of $\pi$ along the central zero of a Gaussian STED beam [64], as shown in Figure 3.5a. Further improvements of STED microscopy involve, e.g., increasing the lateral and axial resolutions by maximizing the localization precision of the excited fluorophore with minimal photon fluxes via MINFLUX [66].

The simultaneous strength and limitation of fluorescence microscopy techniques is that only specifically labeled structures can be imaged. In contrast, X-rays are sensitive to the electron density of the sample. Thus, using X-rays to image a sample yields information about all of its constituents and is not restricted to what is labeled. 


\subsection{X-Ray-Matter Interactions}

X-rays were discovered in 1895 by German physicist Wilhelm Röntgen while he was investigating the physics of a cathode tube in his laboratory [67]. He found that if the cathode tube was sealed in a thick, black container, such that the emitted light was contained, a plate covered with barium platinocyanide placed on the other side of the room became fluorescent. Röntgen showed that new rays of light were produced by the impact of the cathode rays on a material object. As the nature of these new rays was unknown at the time, he gave them the name X-rays.

Within the following sections the terminology and basic concepts of X-ray scattering are introduced and are partially based on Ref. [68]. Readers are referred to Refs. [68-72] for more in-depth reviews. X-rays are elastically scattered by electrons in the atomic shell and, in the scope of this thesis, the first Born approximation is considered. Elastic collisions conserve kinetic energy but alter the propagation direction. The first Born approximation states that only single scattering events can occur, i.e., only the incident wave is scattered. X-rays have typical wavelengths between 1 and $20 \mathrm{~nm}$ and are loosely classified into two regimes: "hard" with $\lambda<2 \mathrm{~nm}$ and "soft", with $\lambda>2 \mathrm{~nm}$.

Consider a monochromatic and parallel beam of X-rays, with initial intensity $I_{0}$ in units of photons per second, being transmitted through matter of thickness $z$. The photons can either interact or pass through the sample unaffected. Photons that interact with the sample are either scattered or absorbed and are subsequently removed from the incident beam, thus the number of photons in the beam decreases. This decrease is termed attenuation and is modeled as:

$$
d I=-\sigma n I d z
$$

where $\sigma$ is the cross-sectional area, which describes the probability of photon interaction, and is expressed in $\mathrm{cm}^{2}$ or barns $\left(1\right.$ barn $\left.=10^{-24} \mathrm{~cm}^{2}\right)$. It is dependent on the chemical composition $Z$ of the material and the energy $E$ of the incident photons. The variable $n$ is the number of atoms per unit volume within the material. The product of $\sigma \times n$ is equal to the linear attenuation factor $\mu$ and has units of $\mathrm{cm}^{-1}$. Substituting $\mu$ into eq. 3.17 and integrating from $z_{\min }=0$ to $z_{\max }=z$ we arrive at the Beer-Lambert attenuation law:

$$
I(z)=I_{0} e^{-(\mu / \rho) \cdot \gamma}
$$

where $\rho$ is the mass density of the material and $\gamma=\rho z$ is the area density. In contrast to eq, 3.8, which describes absorbance, eq. 3.18 describes the continuous intensity decrease. In eq. 3.18 the term $\mu / \rho$ is the mass attenuation coefficient and has units of $\mathrm{cm}^{2} \cdot \mathrm{g}^{-1}$. It 
expresses how easily the volume of a material can be penetrated by the incident beam. Beer-Lambert's law is schematically shown in Figure 3.17.

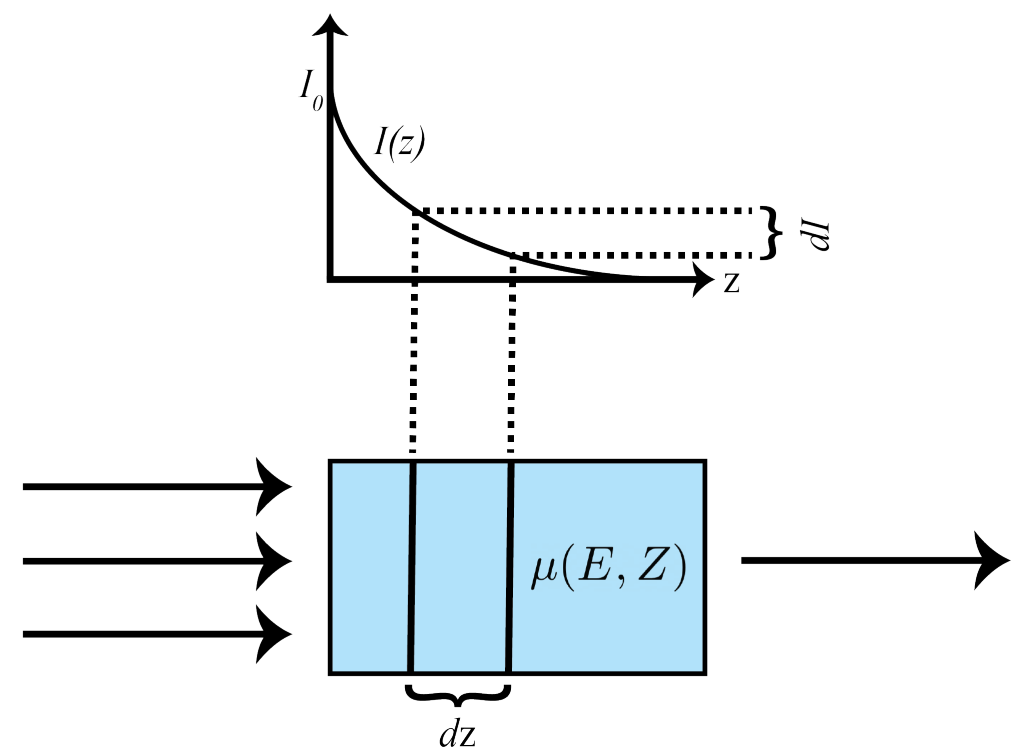

FiguRE 3.6: Illustration of the Beer-Lambert law showing the attenuation of the incident beam along the propagation axis $z$ as it transverses a material with linear attenuation coefficient $\mu$. The number of quanta in a thin layer is proportional to its thickness $d z$. As the beam propagates, its intensity decreases exponentially (eq. 3.18). Figure adapted from Ref. [68].

In the context of imaging with energies up to $100 \mathrm{keV}$, which is relevant for biological imaging, attenuation due to mass is primary caused by three effects: Thomson scattering, Compton scattering and the photoelectric effect. For energies much greater than $100 \mathrm{keV}$, electron-positron pair-production is possible. To account for different interactions between X-rays and matter, the total contribution of each process is summed:

$$
(\mu / \rho)_{\text {Total }}=(\mu / \rho)_{\text {Photoelectric }}+(\mu / \rho)_{\text {Compton }}+(\mu / \rho)_{\text {Thomson }}+(\mu / \rho)_{\text {Pair-production }} .
$$

The mass attenuation coefficient for each process is shown in Figure 3.7 for energies in the range of 1 to $100 \mathrm{keV}$. The specific energy used at the P10 beamline is also shown. The values are calculated using the XCOM: Photon Cross-Sections Database [73] from the National Institute for Standards and Technology. The values are based on a biological sample with an average empirical formula of $\mathrm{H}_{50} \mathrm{C}_{30} \mathrm{~N}_{9} \mathrm{O}_{10} \mathrm{~S}$ [74]. 


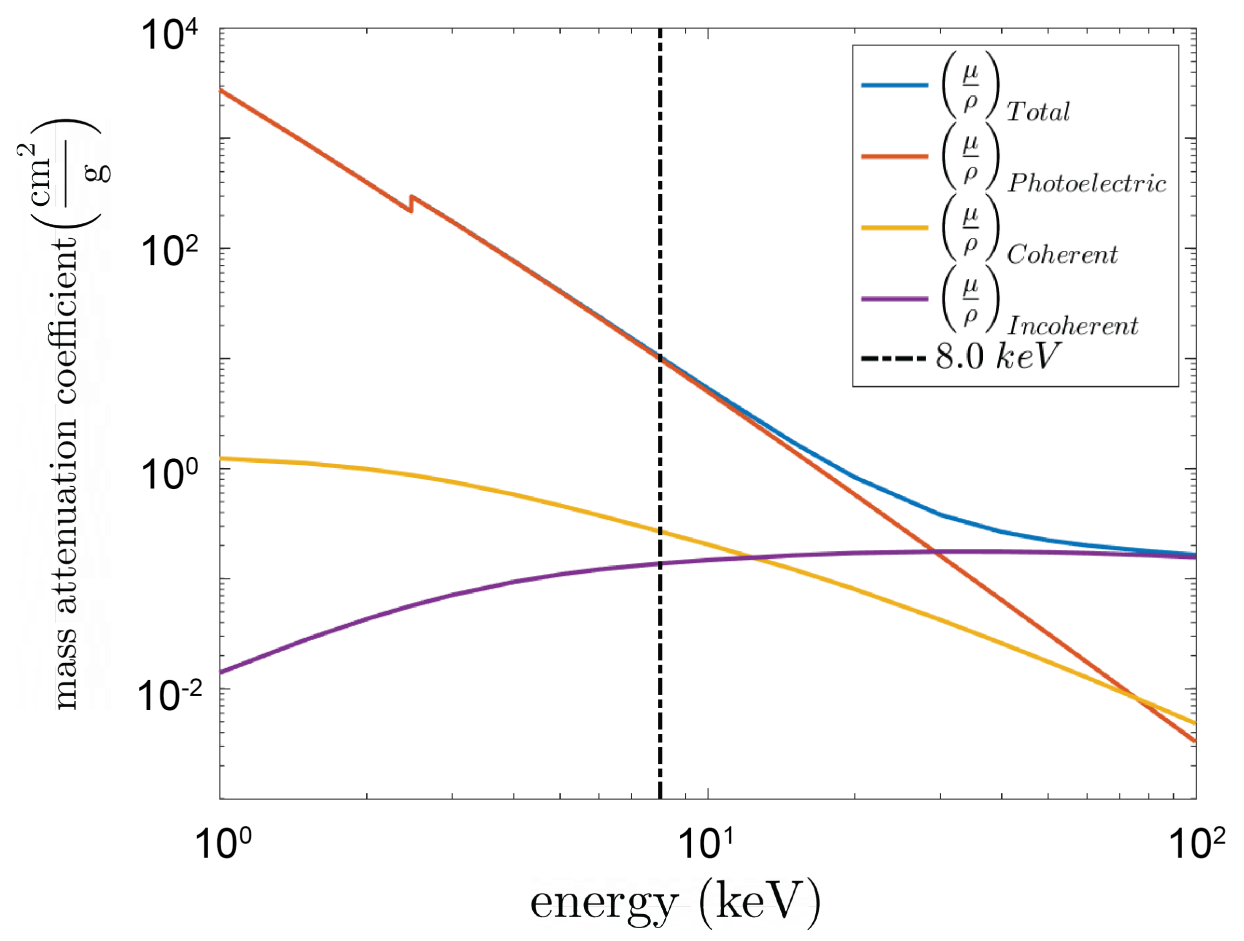

Figure 3.7: Mass attenuation coefficients for a protein with an average empirical formula $\mathrm{H}_{50} \mathrm{C}_{30} \mathrm{~N}_{9} \mathrm{O}_{10} \mathrm{~S}$, showing the contributions for the coherent, incoherent and photoelectric effects. The total of all contributors is shown in blue. The energy ranges from 1 to $100 \mathrm{keV}$ and the energy used for experiments within this work at the P10 beamline is marked at $8.0 \mathrm{keV}$.

\subsubsection{Photon Cross-Section}

The photon cross-section is a measure of how efficiently an atom can scatter a photon at the scattering angle $\theta$ and azimuthal angle $\phi$ into the differential solid angle $d \Omega$, see Figure 3.8. 


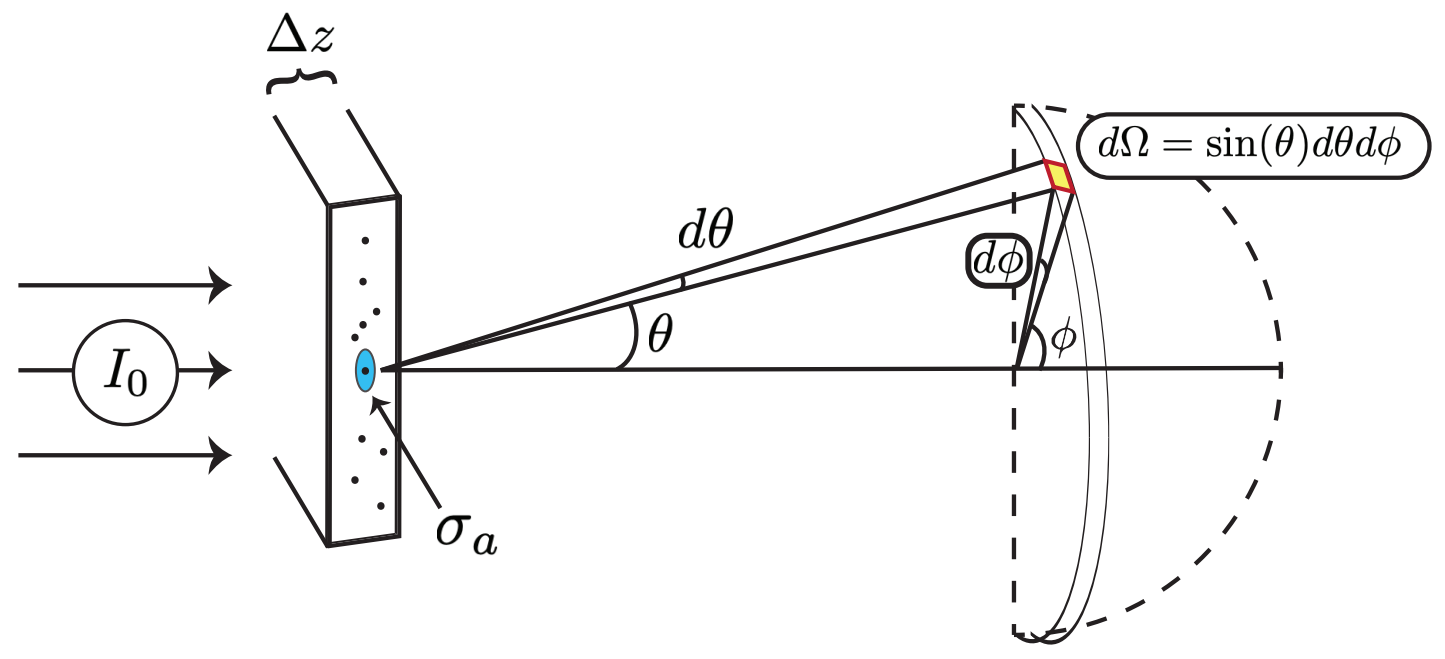

Figure 3.8: Differential cross section schematic. A thin layer of material $\Delta z$ in width is comprised of scatterers (black dots) with uniform electron density. The probability of photons being detected at the scattering angle $\theta$ and azimuthal angle $\phi$ is given by $d \Omega$ (yellow) and is dependent on the cross section $\sigma_{a}$ of each atom (blue).

Consider an X-ray beam with intensity $I_{0}$, in photons per unit time, impinging on an layer with uniform thickness $\Delta z$. The dimensions of the layer are considered to be large enough to intercept the entire beam but thin enough to ensure the beam is not attenuated over $\Delta z$. The intensity of the scattered photons, $I_{s}$, is proportional to $I_{0}$, the number of scatterers $N$ and the solid angle $\Omega$. The solid angle is defined as $\Omega=A / r^{2}$, where $A$ is the surface area of the sphere and $r$ is its radius. Thus, the scattered intensity is given by:

$$
I_{s}=I_{0} N \Omega\left(\frac{d \sigma_{a}}{d \Omega}\right),
$$

where $\sigma_{a}$ corresponds to the photon cross-section of atom $a$. The cross-section is dependent on the incident photon's energy and chemical composition $Z$ of the object, as will be discussed in more detail in the following sections. The differential cross section per atom for the interaction type $j$ is defined as:

$$
\left(\frac{d \sigma}{d \Omega}\right)_{a}^{j}=\frac{\text { number of scattered photons per second into } d \Omega}{I_{0} N d \Omega} .
$$

\subsubsection{Photoelectric Effect}

In 1905, building off the work of Heinrich Hertz [75], Albert Einstein reached the conclusion that the energy of light is not distributed evenly over the classical wavefront but is rather compartmentalized to irreducible finite amounts of energy termed quanta [76]. He asserted that quanta is present within photon-matter interactions, which ultimately 
led him to model the photoelectric effect: When photons impinge on a surface, an electron can absorb the radiation. If the energy of the incident photon is greater than the electron's binding energy the electron can be ejected.

The photoelectric effect occurs for strongly-bonded, i.e., inner-shell, electrons, and is the dominate interaction between photons and atoms at photon energies up to approximately $100 \mathrm{keV}$ (Figure 3.7). If the energy of the incident photon $E_{\gamma}=h c / \lambda=h \nu$ exceeds the binding energy $E_{b}$ of the electron, the photon can be absorbed and the photoelectric effect can occur. If $E_{\gamma} \leq E_{b}$ the photoelectric effect is not possible. The energy of an absorbed photon is transferred to the electron, which is subsequently ejected from the atom with kinetic energy $E=E_{\gamma}-E_{b}$. The ejected electron leaves behind a positively charged ion with a vacancy in an inner shell which can be filled from an electron in an outer-shell. For atoms with a large atomic number $Z$, this filling process can produce fluorescent light in the X-ray regime. Alternatively, the electron which fills the vacancy can transfer the energy $E$ to yet another electron that is subsequently ejected from the atom, termed an Auger electron. Let $N_{k}$ and $1-N_{k}$ denote the probability of fluorescence and Auger electron emission, respectively. The ratio of both probabilities is [68]:

$$
\frac{N_{k}}{1-N_{k}}=\left(-6.4+3.4 Z-0.000103 Z^{3}\right)^{4} \times 10^{-8}
$$

At $Z \approx 30$, the probability of fluorescence and Auger electron emission are approximately equal. The mass attenuation coefficient of $\mathrm{X}$-rays by the photoelectric effect is:

$$
(\mu / \rho)_{\text {photoelectric }} \propto\left(\frac{Z}{h \nu}\right)^{3} .
$$

The cross-section of the photoelectric effect, $\tau^{\text {photoelectric }}$, is given by:

$$
\sigma^{\text {photoelectric }} \propto \frac{Z^{n}}{(h \nu)^{3}}
$$

where $n \approx 4.5$ for light elements and $n \approx 4$ for heavy elements. The strong dependence on $Z$ is the basis for computed tomography based on absorption contrast.

\subsubsection{Compton Scattering}

In 1922 A.H. Compton studied the scattering of X-ray photons, $\gamma$, by graphite and observed that spectra measured at various angles $\theta_{\gamma}$ contained two peaks; one corresponding to the wavelength $\lambda$ of the incident photon, which can be explained by elastic scattering, and another peak corresponding to a larger wavelength $\lambda^{\prime}>\lambda$, where $\Delta \lambda=\lambda^{\prime}-\lambda$ increased with increasing $\theta_{\gamma}[77]$. Compton concluded the second peak is caused by an inelastic scattering process. 
Compton was able to explain his observation by treating photons as quantum-mechanical particles which undergo collisions with electrons and thus lose part of their kinetic energy. Since the binding energies of low- $Z$ elements are negligible compared to typical X-ray photon energies, it is assumed the target electron is initially free and at rest. When an incident photon with energy $E_{\gamma}=h \nu$ impinges on the target electron, the energy difference between it and the scattered photon, with $E_{\gamma}^{\prime}=h \nu^{\prime}$, is transferred to the target electron in the form of kinetic energy and momentum. If the transferred energy is greater than approximately $100 \mathrm{keV}$ the electron can be ejected with energy $E_{e}^{\prime}$. The collision process is schematically shown in Figure 3.9.

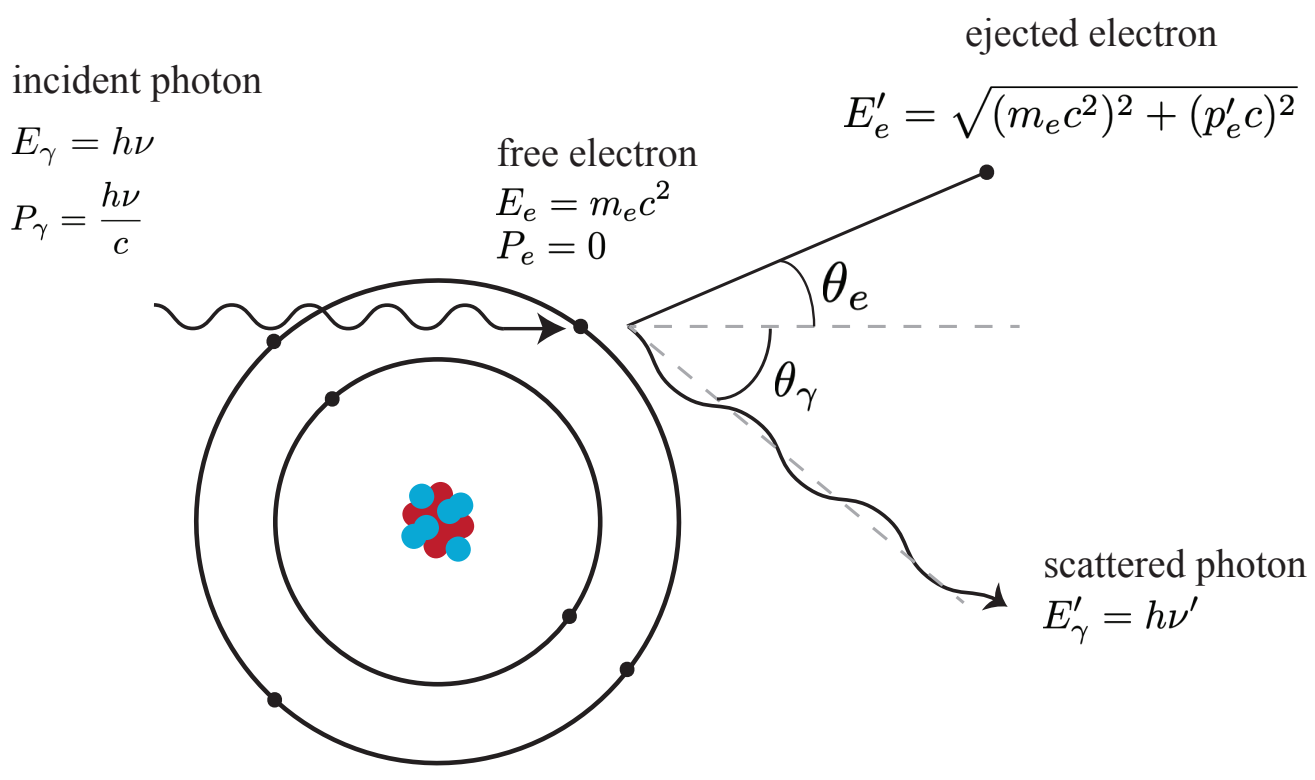

Figure 3.9: Schematic of Compton scattering. An incident photon with energy $E_{\gamma}$ and momentum $P_{\gamma}$ impinges on a free and weakly-bound target electron that is initially at rest. If the transferred energy is greater than approximately $100 \mathrm{keV}$ the target electron can be ejected with energy $E_{e}^{\prime}$ and momentum $P_{e}^{\prime}$. Figure adapted from Ref. [68].

The energy of the scattered photon is given by:

$$
h \nu^{\prime}=\frac{h \nu}{1+\frac{h \nu}{m_{e} c^{2}}\left(1-\cos \theta_{\gamma}\right)},
$$

where $m_{e}$ is the electron mass and $c$ is the speed of light in vacuum. The mass attenuation coefficient for X-rays by Compton scattering is approximately constant and is given by:

$$
(\mu / \rho)_{\text {Compton }} \propto \frac{Z}{A} \approx 0.5
$$

where $Z$ and $A$ are the atomic number and atomic mass number, respectively. The cross section of the Compton effect, $\sigma_{C}$, is proportional to $Z$ [68]. 


\subsubsection{Thomson Scattering}

Named after J.J. Thomson [78], who is also credited with discovering the electron, Thomson scattering is an elastic scattering process between electromagnetic radiation and a free electron. It is a low-energy variant of Compton scattering where the kinetic energy transmitted to the free electron is neglected, thus the wavelength of the incident and scattered photons are the same, as shown in Figure 3.10.

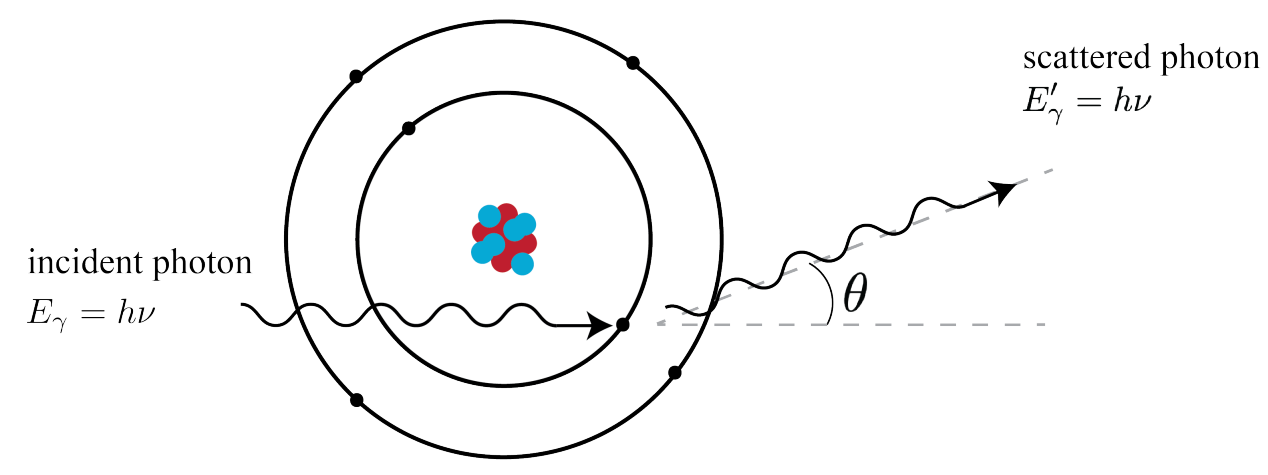

Figure 3.10: In Thomson scattering, an X-ray photon with incident energy $E_{\gamma}$ is elastically scattered at an angle $\theta$ by a free electron. The energy $E_{\gamma}^{\prime}$ of the scattered photon is equal to $E_{\gamma}$.

Although Thomson scattering accounts for a small contribution to the overall attenuation of an X-ray beam (Figure 3.7), is critical for the underlying processes of both $\mathrm{X}$-ray scattering and X-ray phase contrast imaging. To show Thomson scattering is a low-energy variant of Compton scattering we first begin with eq. 3.25 and make the substitution $\alpha=\frac{h \nu}{m_{e} c^{2}}$ :

$$
h \nu^{\prime}=\frac{h \nu}{1+\alpha\left(1-\cos \theta_{\gamma}\right)} .
$$

For photons with energies below $h \nu \ll 511 \mathrm{keV}$, where $511 \mathrm{keV}$ is the electron rest mass, $\alpha \sim 0$, thus:

$$
h \nu^{\prime}=h \nu .
$$

The incident and scattered photon thus have the same wavelength. The cross-section for Thomson scattering by a single, free electron is given by:

$$
\sigma_{T}=\frac{8 \pi r_{0}^{2}}{3}=0.67 \times 10^{-28} \mathrm{~m}^{2}
$$

where $r_{0}$ is the classical electron radius:

$$
r_{0}=\frac{1}{4 \pi \epsilon_{0}} \cdot \frac{e^{2}}{m_{e} c^{2}}=2.818 \times 10^{-15} \mathrm{~m}
$$


As can be seen in eq. 3.29, the cross section for Thomson scattering is independent of the photon energy $h \nu$ and the strong dependence to the electron's charge and mass indicates that scattering from protons or atomic nuclei can be neglected.

\subsubsection{Electron-Positron Pair-Production}

In this process a photon with an energy greater than $h \nu>2 m_{e} c^{2}$ can interact with the Coulomb field surrounding a charged particle and generate an electron-positron pair, resulting in each to have an energy of $h \nu \simeq m_{e} c^{2}$. The mass attenuation coefficient of $\mathrm{X}$-rays due to pair-production is:

$$
(\mu / \rho)_{\text {Pair-Production }} \propto Z \cdot \ln (h \nu) .
$$

The cross section of the pair-production process is given by:

$$
\sigma \propto Z^{2} \cdot \ln (h \nu)
$$

For photon energies smaller than $h \nu=2 m_{e} c^{2}$ the electron-positron pair-production cannot occur. Thus, in the scope of this thesis, this process can be neglected since energies in the keV range are used.

\subsubsection{Rayleigh Scattering}

Named after Lord Rayleigh (John William Strutt) [79], Rayleigh scattering is solely an elastic scattering process which occurs when the incident photon's wavelength is much larger than the size of the object (atom). When the photon impinges on the atom, it's elastically scattered by the atom's electron cloud and thus retains its energy. In the visible light regime the strong dependence on the wavelength $\left(\sim \lambda^{-4}\right)$ indicates shorter, blue light is more susceptible to scattering than longer, red light. Thus Rayleigh scattering is responsible for the sky appearing blue.

In the hard X-ray regime, where $\lambda \approx \AA$ and is thus similar to the size of an atom, Rayleigh scattering can be neglected. However, in the soft X-ray regime where $\lambda \approx n m$, Rayleigh scattering becomes more probable and should be considered. The mass attenuation coefficient of X-rays due to Rayleigh scattering is:

$$
(\mu / \rho)_{\text {Rayleigh }} \propto \frac{Z}{(h \nu)^{2}} .
$$


The cross-section of Rayleigh scattering is expressed as $\sigma_{R}$ :

$$
\sigma_{R} \propto\left(\frac{Z}{h \nu}\right)^{2}
$$

\subsection{Kinematic Theory of Diffraction}

So far, various scattering processes have been discussed when taking into consideration a single atom. Now, consider a crystal comprised of two planes of multiple atoms, as shown in Figure 3.11a. In this scenario, as the sample is a well-defined crystal, we do not speak of scattering but diffraction. In particular, we are speaking of kinematic diffraction as the assumption that each diffraction event is independent of any other event is held.
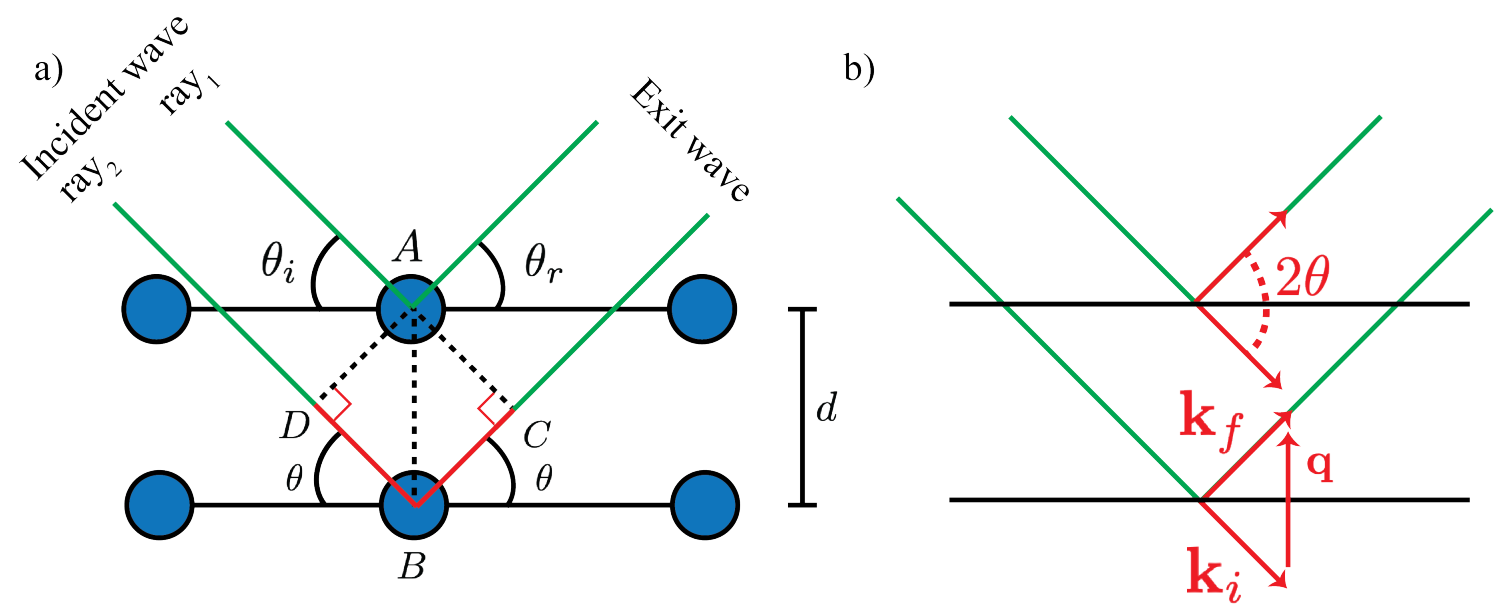

FiguRE 3.11: a) Geometric representation of Bragg's law. Each blue sphere represents an atom in planes spaced $d$ apart. b) Construction of the momentum transfer $\boldsymbol{q}$, equal to the difference between the incident wavevector $\mathbf{k}_{\mathbf{i}}$ and exit wavevector $\mathbf{k}_{\mathbf{f}}$. The scattering angle is denoted as $\theta$.

The incident wave is described by the wavevector $\mathbf{k}_{i}$ and the exit wave by the wavevector $\mathbf{k}_{f}$, as depicted in Figure 3.11b. The difference between the two yields the momentum transfer $\boldsymbol{q}$ :

$$
\boldsymbol{q}=\mathbf{k}_{f}-\mathbf{k}_{i}
$$

In 1913, William and Lawrence Bragg [80] proposed a method to analyze the diffraction of X-rays from such a crystalline structure. For adjacent atoms in a single plane, e.g., the upper lattice plane in Figure 3.11a, diffracted X-rays will constructively interfere if the angle of incidence $\theta_{i}$ equals the angle of reflection $\theta_{r}$. For atoms in successive planes, diffracted X-rays will only constructively interfere at an angle $\theta$ if the difference in path length between ray 1 and ray 2, given by the sum of the vectors DB and BC, 
is an integer number $n$ of wavelength $\lambda$. In other words, constructive interference will only occur when:

$$
\mathrm{DB}+\mathrm{BC}=n \lambda \quad \text { where } n=1,2,3 \ldots
$$

As the path length of $D B=B C=d \sin (\theta)$, it follows that:

$$
n \lambda=2 d \sin (\theta) \text { where } n=1,2,3 \ldots
$$

where $d$ is the distance between planes and $\theta$ is the diffraction angle. Eq. 3.37 is referred to as Bragg's law.

The recorded intensity $I(\boldsymbol{q})$ corresponds only to the square modulus of the amplitude of the incoming wave:

$$
I(\boldsymbol{q})=|\psi(\boldsymbol{q})|^{2}=\psi(\boldsymbol{q}) \psi^{*}(\boldsymbol{q}),
$$

where $\boldsymbol{q}$ is the momentum transfer and the amplitude of the scattered wave $\psi(\boldsymbol{q})$ by one atom is expressed as:

$$
\psi(\boldsymbol{q})=f \mathrm{e}^{2 \pi i \mathbf{q r}}
$$

The atomic scattering factor $f$ represents the value of the electron cloud density in reciprocal space and is expressed as:

$$
f=\int_{0}^{\infty} \rho(\mathbf{r}) d \mathbf{r}
$$

where $\mathbf{r}=(x, y, z)$ is the position of the atom and $\rho(\mathbf{r})$ is the spatial density of its electron cloud. Thus as $\boldsymbol{q} \longrightarrow 0, \rho(\mathbf{r}) \longrightarrow Z$, the atomic number. As $\boldsymbol{q} \longrightarrow \infty, \rho(\mathbf{r}) \longrightarrow 0$. For a material that is composed of a group of atoms, of either the same or different type, their positions with respect to the same origin $\mathbf{r}_{n}$ of a unit cell must be taken into account, where $n$ denotes the $n^{\text {th }}$ atom. A unit cell defines the basic 3-dimensional structure of the lattice which regularly repeats itself to form the overall structure of the crystal. It is defined with vectors $\mathbf{u}_{1}, \mathbf{u}_{2}$ and $\mathbf{u}_{3}$. The position of the $n^{\text {th }}$ atom with respect to $\mathbf{r}_{n}$ is denoted as $\mathbf{d}_{n}$. Therefore, eq. 3.39 can be rewritten to account for all atoms:

$$
\psi(\boldsymbol{q})=\psi_{0} \sum_{n} f_{n} \mathrm{e}^{2 \pi i \mathbf{q} \mathbf{d}_{n}} \sum_{m_{1}} \mathrm{e}^{2 \pi i m_{1} \mathbf{q u}_{1}} \sum_{m_{2}} \mathrm{e}^{2 \pi i m_{2} \mathbf{q u}_{2}} \sum_{m_{3}} \mathrm{e}^{2 \pi i m_{3} \mathbf{q u} \mathbf{u}_{3}},
$$

where $m_{i} \in \mathbb{N}$ indicates the position of the $m_{t h}$ cell in the crystal. The first term of eq. 3.41 is termed the form factor $F(\boldsymbol{q})$ and defines the structure of the unit cell:

$$
F(\boldsymbol{q})=\sum_{n} f_{n} \mathrm{e}^{2 \pi i \mathbf{q d} \mathbf{d}_{n}}
$$


The remaining three sums of eq. 3.41 are dependent on the 3-dimensional shape of the crystal. They are collectively referred to as the structure factor $S(q)$ :

$$
S(\boldsymbol{q})=\sum_{\mathbf{u}_{i}} \mathrm{e}^{2 \pi i \boldsymbol{q u} \mathbf{u}_{i}} \quad \text { where } \quad i=1,2,3
$$

Thus, the intensity recorded by the detector can be rewritten as:

$$
I(\boldsymbol{q})=|\psi(\boldsymbol{q})|^{2}=|F(\boldsymbol{q}) S(\boldsymbol{q})|^{2} .
$$

The form factor and structure factor are represented by the flower and dots, respectively, in Figure 3.12

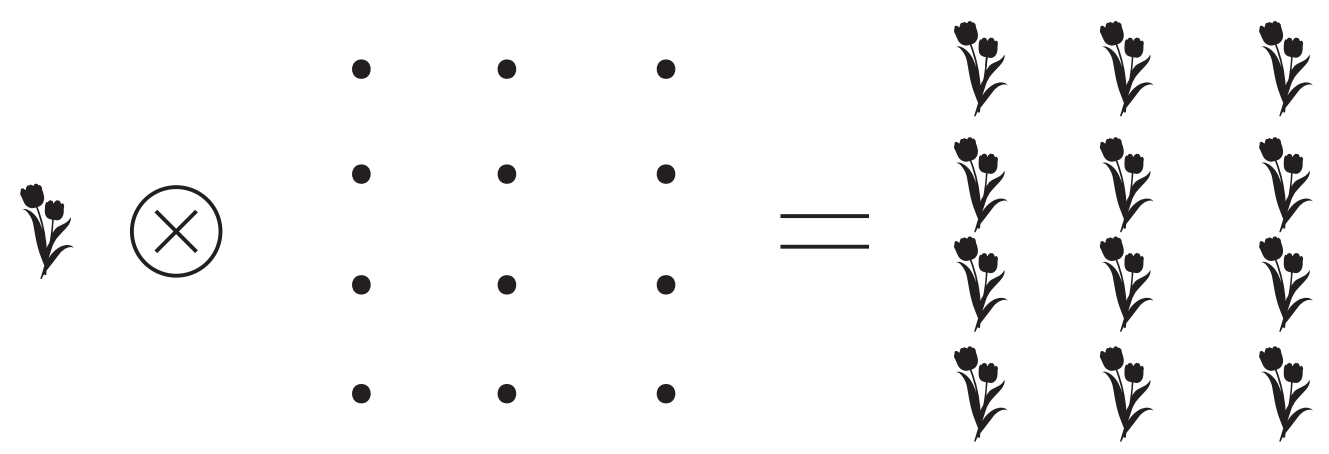

FiguRE 3.12: Representation of the from and structure factors, denoted by the silhouette of the flowers and black dots, respectively. The convolution of both factors yields the crystal structure.

\subsubsection{Form Factor}

As described by eq. 3.42 and shown in Figure 3.12, the form factor $F(\boldsymbol{q})$ is dependent on the size and shape of the sample. For non-crystalline samples $F(\boldsymbol{q})$ describes the shape of the scatterers averaged over all orientations. Consider a sample comprised of monodisperse spheres with radius $R$. The spheres are assumed to have uniform electron density $\rho(\boldsymbol{r})=\rho$. For such a system $F(\boldsymbol{q})$ can be analytically written as:

$$
F(\boldsymbol{q})=\int_{0}^{\infty} \rho(\boldsymbol{r}) \mathrm{e}^{i \boldsymbol{q} \boldsymbol{r}} d \boldsymbol{r}
$$

A volume element of a sphere is defined as $d V=r^{2} \sin (\theta) d \theta d \phi d r$. Writing the scalar product $\boldsymbol{q} \boldsymbol{r}=q r \cos (\theta)$, eq. 3.45 can be rewritten as:

$$
F(q)=\rho \int_{0}^{R} \int_{0}^{2 \pi} \int_{0}^{\pi} \mathrm{e}^{i q r \cos (\theta)} r^{2} \sin (\theta) d \theta d \phi d r .
$$


Performing the integrations yields the form factor:

$$
F(q)=\frac{3[\sin (q R)-q R \cos (q r)]}{(q R)^{3}} .
$$

Eq. 3.47 is valid only for a system comprised of spheres with radius $R$. Derivations of form factors corresponding to a variety of systems can be found in [81]. Figure 3.13 a shows the calculated form factors for systems comprised of spheres $(R=25 \mathrm{~nm})$, infinitely thin disks $(R=25 \mathrm{~nm})$, infinitely thin rods (length $L=25 \mathrm{~nm})$ and cylinders $(R=25 \mathrm{~nm}, L=50 \mathrm{~nm})$. If the particles in the sample are not monodisperse, i.e., they have different sizes and/or shapes, the system is polydisperse and the form factors of all particles are summed [71]. Figure 3.13b shows the effects of polydispersity: $F(q)$ is plotted for systems of monodisperse spheres with radius $R$ and the average is taken to mimic the effects of polydispersity.

a)

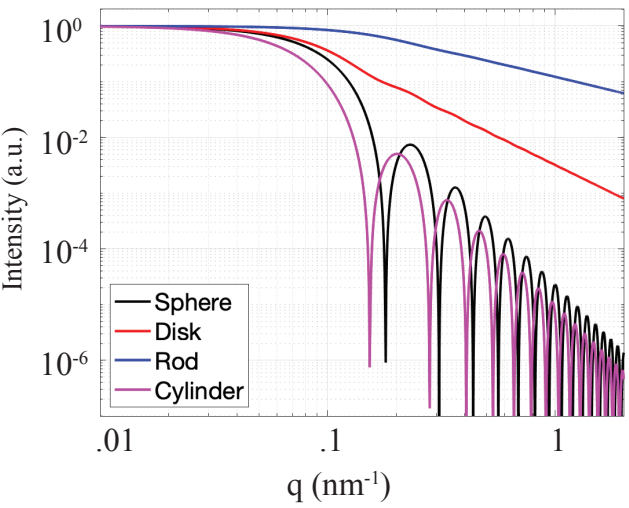

b)

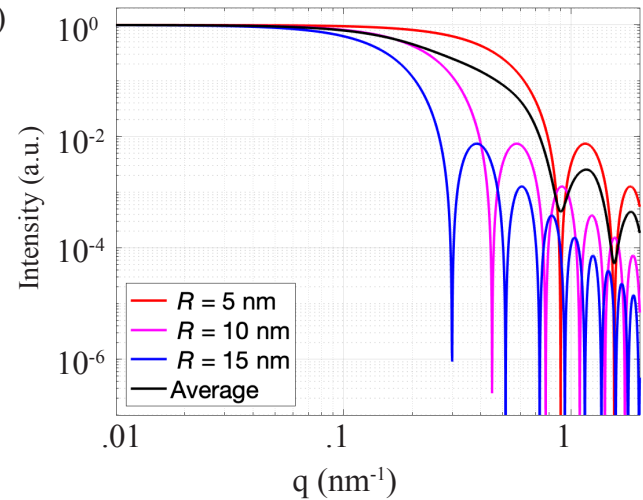

Figure 3.13: Form factors $F(q)$ of various objects. a) The form factor of a sphere (black, $R=25 \mathrm{~nm}$ ), infinitely think disk (red, $R=25 \mathrm{~nm}$ ), infinitely thin rod (blue, $L=25 \mathrm{~nm}$ ) and cylinder (magenta, $R=25 \mathrm{~nm}, L=50 \mathrm{~nm}$ ). b) The form factor of monodisperse spheres with different radii. To mimic the effect of polydispersity, the average is calculated.

\subsubsection{Structure Factor}

The structure factor $S(q)$, also termed the lattice symmetry, describes the scattering amplitude and is dependent on the arrangement of the scatterers of the object. For a perfect, infinite crystal, $S(q)$ can be expressed as a Dirac delta function whose width is inversely proportional to the number of atoms. For liquid samples, where no regular lattice is defined, the structure factor peak is broaden and describes the interaction potential between two particles. 
To conclude this chapter, Bragg's law and the kinematic theory of diffraction served as the foundation necessary for André Guinier to develop small-angle scattering (SAS) in the 1930's. Today, SAS is widely used in both small angle neutron scattering (SANS) and small angle X-ray scattering (SAXS). In contrast to crystal diffraction, SAS techniques probe non-crystalline structures including, but not limited to, bone, teeth, wood and whole cells. In chapter 4 , a description of the experimental methods used in thesis are introduced. The applications of scanning SAXS, X-ray holography and X-ray tomography are discussed, as well as the experimental setups used at the coherence application P10 beamline at DESY and the approaches used to analyze the acquired data. The design of a custom-built, beamline-compatible STED microscope is also introduced. Chapter 4 is intended to give readers the information necessary for interpreting how the data were acquired and analyzed. 



\subsection{Sample Preparation}

\subsubsection{Cell Culture on Silicon-Nitride Membranes}

A large portion of measurements performed within this work are carried out on lyophilized NIH-3T3 fibroblast cells [82] adhered on a silicon-rich nitride $\left(\mathrm{Si}_{3} \mathrm{~N}_{4}\right)$ membrane. Initially, the cells are cultured in a $25 \mathrm{~cm}^{2}$ cell-culture flask (Nunc A/S, Roskilde, Denmark) using high-glucose $\left(4.5 \mathrm{~g} \cdot \mathrm{mL}^{-1}\right)$ Dulbecco's Modified Eagle's Medium (DMEM, D6429, Lot RNBF508, Sigma-Aldrich, Taufkirchen, Germany) supplemented with $10 \%$ (v/v) fetal bovine serum (FBS. F0804, Lot 015M3344; Sigma-Aldrich) and 100 units $\cdot \mathrm{mL}^{-1}$ penicillin and $0.1 \mathrm{~g} \cdot \mathrm{L}$ streptomycin. The cells are stored in a water-saturated incubator kept at $37^{\circ} \mathrm{C}$ with $5 \% \mathrm{CO}_{2}$. Once the cells reach a confluency of $\sim 80 \%$ they are passaged. To passage the cells, the cell suspension is discarded and the cells are gently washed, three times, with $4 \mathrm{~mL}$ phosphate buffered saline (PBS. Sigma-Aldrich) supplemented with $0.02 \%$ (w/v) ethylenediaminetetraacetic acid (EDTA, 8040.2, Carl Roth, Karlsruhe, Germany). The cells are detached from the flask via trypsin incubation $\left(37^{\circ} \mathrm{C}, 5 \% \mathrm{CO}_{2}\right)$ for 150 seconds using $1 \mathrm{~mL}$ of $0.05 \%$ trypsin derived from porcine pancreas (T47995G, Sigma-Aldrich). $4 \mathrm{~mL}$ of medium is added to the flask and the suspension is gently syphoned several times to facilitate the detachment process. $3 \mathrm{~mL}$ of the suspension is added to a new flask containing $2 \mathrm{~mL}$ medium, thus ending the passaging process. The passaging process typically occurs every 3 to 4 days, depending on cell proliferation. 
For most experiments, cells are cultured on $\mathrm{Si}_{3} \mathrm{~N}_{4}$ windows with a membrane size and thickness of $1.5 \times 1.5 \mathrm{~mm}^{2}$ and $1 \mu \mathrm{m}$, respectively, and a frame size and thickness of $5 \times 5 \mathrm{~mm}^{2}$ and $200 \mu \mathrm{m}$, respectively (Silson Ltd, Warwickshire, England). These windows are used here as substrates for two primary reasons: they have low X-ray absorption and NIH-3T3 fibroblast cells adhere to the membrane. To render the windows hydrophilic, thus promoting cell adhesion, the windows are plasma cleaned using a radio frequency power of $18 \mathrm{~W}$ for $30 \mathrm{~s}$ (Harrick Plasma, PDC-32G, Ithaca, New York). Each window is then placed in a $3 \mathrm{~cm}$ Petri dish and $1.7 \mathrm{~mL}$ of medium is subsequently added. Care is taken to ensure no air bubbles are present. $300 \mu \mathrm{L}$ of cell suspension, with a typical concentration of $\sim 3.8 \times 10^{5}$ cells $\cdot \mathrm{mL}^{-1}$, is removed from the cell-culture flask and is placed into the Petri dish containing the $\mathrm{Si}_{3} \mathrm{~N}_{4}$ window. Note that a portion of measurements within this thesis include imaging fluorescently labeled cells in the hydrated and lyophilized states. It is at this step in the cell culture process where the alkyne is introduced in the cells (see section 4.1.2 or A.2 in the appendix). For both labeled and non-labeled cells, the Petri dish is placed into the incubator chamber for roughly 48 hours to promote cell proliferation and adhesion on the window. The cells are then gently washed with PBS and chemically fixed at room temperature using $3.7 \%$ formaldehyde supplemented with $1 \%$ methanol for 15 minutes. Non-labeled cells are placed in $2 \mathrm{~mL}$ PBS and stored at $4{ }^{\circ} \mathrm{C}$. Labeled cells are first permeabilized with Triton X-100 (Sigma-Aldrich) prior to being stored in PBS at $4{ }^{\circ} \mathrm{C}$. The general process of culturing cells on a $\mathrm{Si}_{3} \mathrm{~N}_{4}$ window is schematically shown in the red column of Figure 4.3 .

To image (fluorescently labeled) hydrated cells, the cells are not cultured on a $\mathrm{Si}_{3} \mathrm{~N}_{4}$ window but rather a $3 \mathrm{~cm}$ diameter, $1 \mu \mathrm{m}$ thick polypropylene foil. The culturing process follows the protocol described above. By encapsulating the cells with $300 \mu \mathrm{L}$ PBS between two polypropylene rings, a liquid chamber is built. The benefit of using polypropylene foil to manufacture a liquid chamber is that it is more rigid than a comparable $\mathrm{Si}_{3} \mathrm{~N}_{4}$ chamber and thus facilitates the sample-handling and technical aspects when imaging hydrated samples. Polypropylene rings are manufactured by Jochen Herbst from the Institute for X-Ray Physics at the University of Göttingen. Cells encapsulated between polypropylene rings is schematically shown in Figure 4.1. 
a)

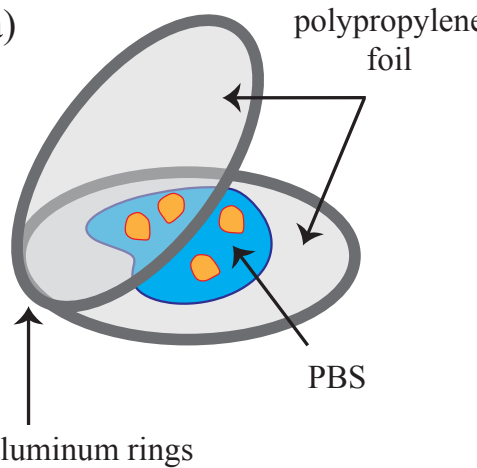

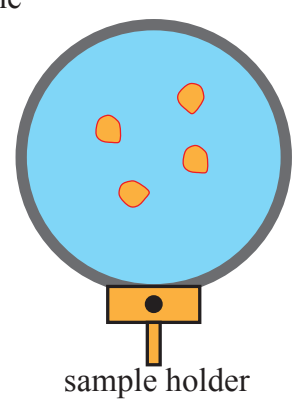

b)

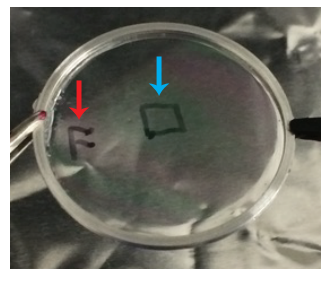

FiguRE 4.1: Sample preparation for hydrated cells. a) Cells (yellow) are cultured on a $3 \mathrm{~cm}$ diameter, $1 \mu \mathrm{m}$ thick polypropylene foil. By adding a small amount of PBS and gluing a second ring on top, a liquid-encapsulation chamber is built. b) Photograph of the resulting chamber. The black square (blue arrow) and "F" (red arrow) are used as references when locating cells.

\subsubsection{Fluorescent Labeling of DNA}

Labeling of DNA is performed using the Click-iT EdU Alexa Fluor 647 (C10340, Thermo Fisher Scientific) imaging kit. Detection of the fluorophore is based on a "Click" [83] reaction, a copper-catalyzed covalent reaction between an azide and alkyne via a process called bioconjugation. In the Click-iT EdU cell proliferation assay EdU (5-ethynyl-2'deoxyuridine), a nucleoside analog of thymidine, is incorporated into DNA during DNA synthesis. In this application, the EdU contains the alkyne and the Alexa Fluor 647 dye contains the azide. The dye has a peak excitation and emission wavelength of $\lambda_{\text {ex }}=$ $650 \mathrm{~nm}$ and $\lambda_{\mathrm{em}}=668 \mathrm{~nm}$, respectively. Figure 4.2 a shows an overlay of an epifluorescent and visible-light phase contrast image of fluorescently labeled cells adhered on a $\mathrm{Si}_{3} \mathrm{~N}_{4}$ window, and Figure 4.2b shows a zoom-in of a single cell from the same window. Both images are acquired using a Zeiss AXIO Observer Z.1 microscope equipped with a $40 \times$ phase contrast objective. Confocal and STED micrographs of the single cell shown in Figure 4.2b are shown in Figure 4.2c and Figure Figure 4.2d, respectively. The protocol used for labeling is found in Appendix A.2. 
a)

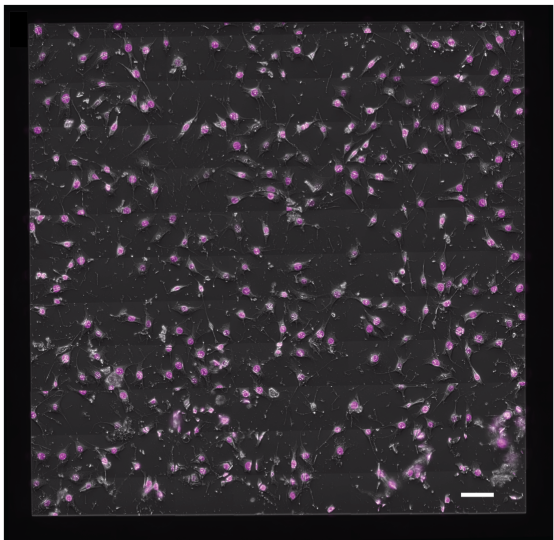

c)

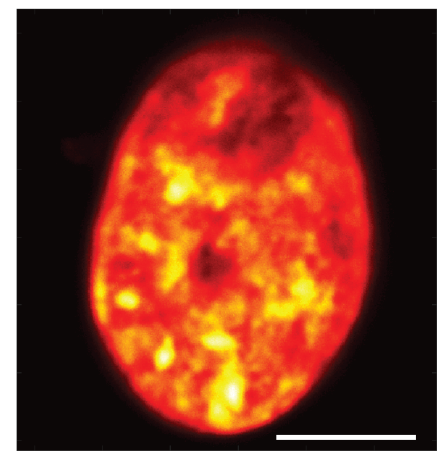

b)

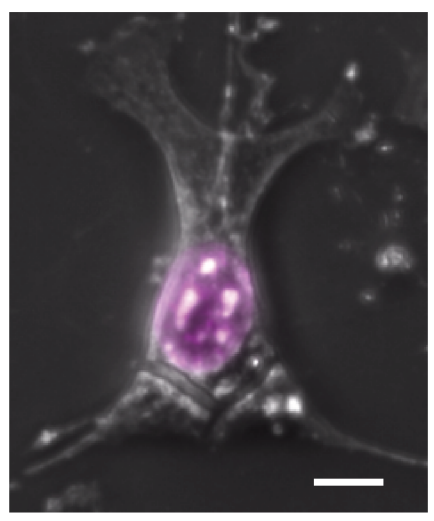

d)

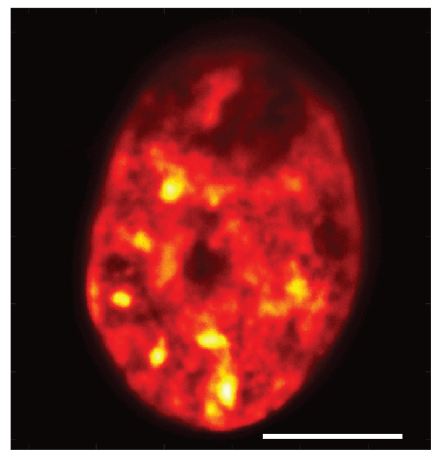

Figure 4.2: Fluorescently labeled nuclei of $3 \mathrm{~T} 3$ fibroblasts adhered to a $\mathrm{Si}_{3} \mathrm{~N}_{4}$ membrane. a) Epifluorescent image overlaid on a visible-light phase contrast image. The scale bar is $100 \mu \mathrm{m}$. b) Zoom-in of a single cell from a). c) Confocal and d) STED images of the nucleus shown in b). The scale bars in b), c) and d) are $10 \mu \mathrm{m}$.

\subsubsection{Grid Plunging and Lyophilization}

For all non-hydrated measurements performed in this thesis, the cells are lyophilized (freeze-dried). Cells are composed of elements with low atomic numbers. Consequently, the scattering power of X-rays is relatively weak, leading to poor contrast (see section 4.2.1). To help mitigate these effects, the cells are lyophilized. Replacing the water in the sample with air increases the electron density contrast between the sample and background, thus improving image contrast. After the cells, either labeled or unlabeled, have been chemically fixed, they are lyophilized.

The process begins by removing a $\mathrm{Si}_{3} \mathrm{~N}_{4}$ window from the $4{ }^{\circ} \mathrm{C}$ fridge and gently rinsing the entire window with ultra-pure Milli-Q water (Millipore Sigma, Burlington, Massachusetts). The window is then placed into the water-saturated chamber of a commercially available Leica EM GP grid plunger (Leica Microsystems, Wetzlar, Germany) using a pair of ultra-fine tweezers. To ensure no ice structures are formed when starting the lyophilization process, the temperature and relative humidity of the chamber is kept 
at $20^{\circ} \mathrm{C}$ and $99 \%$, respectively. Theultra-pure water on both sides of the $\mathrm{Si}_{3} \mathrm{~N}_{4}$ window is manually blotted with filter paper and the window is then rapidly plunged $(\sim 200 \mathrm{~ms})$ into a bath of liquid propane and ethane. The bath consists of $63 \%$ propane and $37 \%$ ethane; both have a purity of N35 (Air Liquide, Paris, France). The temperature of the bath is kept at $-196^{\circ} \mathrm{C}$. The process of rapidly cooling the cell ensures the vitrification of the residual ultra-pure water, thus creating an amorphous layer which preserves all structures within the cell. The $\mathrm{Si}_{3} \mathrm{~N}_{4}$ windows are transferred to a liquid nitrogen $\left(\mathrm{LN}_{2}\right)$ dewar where they are stored until the lyophilization process begins. The lyophilization process is performed by Jochen Herbst and incorporates the use of a home-built vacuum chamber. The lyophilization process is schematically shown in the blue columns of Figure 4.3. The green column in Figure 4.3 shows photos of the sample in the beamline at the P10 beamline at DESY. See Appendix A.1 for a more detailed description of the grid plunging process.

\section{Biological \\ Preparation}

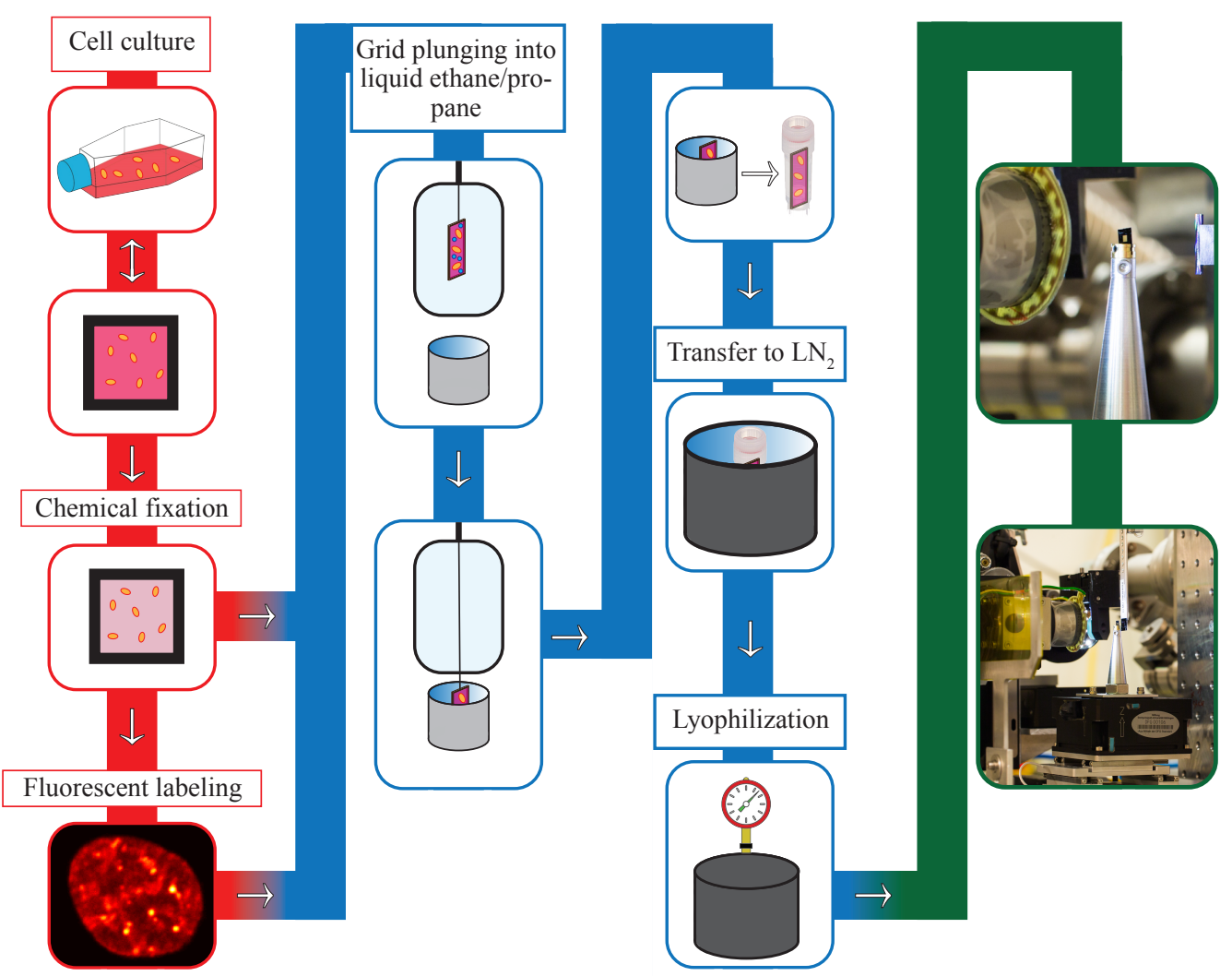

Physical

Preparation

\section{X-ray \\ Measurements}

Figure 4.3: Schematic overview of preparing cells on $\mathrm{Si}_{3} \mathrm{~N}_{4}$ windows. Biological Preparation: The cells are cultured on $\mathrm{Si}_{3} \mathrm{~N}_{4}$ windows, chemically fixed and, if applicable, fluorescently labeled. Physical Preparation: The windows are grid plunged, stored in $\mathrm{LN}_{2}$ and lyophilized. X-ray Measurements: After lyophilization, the samples are ready to be mounted at the beamline. 


\subsection{Small-Angle X-ray Scattering}

Small-Angle X-ray Scattering (SAXS) is a technique used to study structural features of colloidal size ( $\sim 10 \mathrm{~nm}$ to $1 \mu \mathrm{m})$. It was developed in the 1930's by André Guinier [69] and Otto Kratky [70], who collectively introduced the theoretical, experimental and data analysis aspects. As colloidal dimensions are much larger than the wavelength typically used for X-ray measurements $\left(\lambda=0.154 \mathrm{~nm}\left(\mathrm{CuK}_{\alpha}\right)\right)$, the angular range of observable scattering is small and ranges from $\sim 0.1^{\circ}$ to $10^{\circ}$. As X-rays are primarily scattered by electrons, a SAXS signal is only observed when electron density inhomogeneities exist within a sample. SAXS yields access to structural parameters such as the size, shape, aggregation state, morphology and specific surface of the sample. As such, SAXS can be applied to a wide range of samples including, but not limited to: colloids, polymers and proteins, as well as samples in the dry, liquid or powder state.

For SAXS measurements, the two-dimensional scattering pattern recorded by the detector is azimuthally integrated and the resulting radial intensity profile $I$ is plotted against the magnitude of the scattering vector $\mathbf{q}$ (see eq. 3.35):

$$
q=\frac{4 \pi}{\lambda} \sin (\theta)
$$

where $2 \theta$ is the scattering angle and $\lambda$ is the wavelength of the incident X-rays. An example of a radial intensity profile $I(q)$ is shown in Figure 4.5g. The radial intensity profile is the starting point for a majority of the analysis performed in SAXS experiments and $I \propto q^{-\alpha}$, where the exponent $\alpha$ is related to the morphology of the scatterers.

\subsubsection{Contrast Formation in SAXS}

Contrast is used to describe the amplitude of scattered radiation of a specific object compared to the that of the average object, thus describing how easily the specific object "stands out" against the average. In SAXS, the amplitude of scattered light is governed by the scattering length $f_{X}$, which is a measure of the scattering power of a material. The scattering length of scattered light by each atom of the sample is given by:

$$
f_{X}=r_{0} N_{\mathrm{e}^{-}}
$$

where $r_{0}$ is the classical electron radius (eq. 3.30) and $N_{\mathrm{e}^{-}}$is the number of electrons for the atom. For X-rays, $f_{X}$ is dependent on the electron density $\rho$ distribution within the sample and thus increases with the atomic number $Z$. The scattering length values of various atoms found in biological matter are presented in Table 4.1. The values are obtained from the National Institute of Standards and Technology. Note that as the 
atomic number increases the scattering length increases. It is for this reason that the scattering power of X-rays by biological material is much less than, e.g., metals.

TABLE 4.1: Scattering length values $f_{X}$ of X-rays for atoms found within biological samples. The atomic mass is denoted as $u$ and the number of electrons as $N_{\mathrm{e}^{-}}$. The scattering length is given in femtometers $\left(10^{-15} \mathrm{~m}\right)$.

\begin{tabular}{|c|ccccccccc|}
\hline Atom & $\mathrm{H}$ & $\mathrm{C}$ & $\mathrm{N}$ & $\mathrm{O}$ & $\mathrm{Na}$ & $\mathrm{Mg}$ & $\mathrm{S}$ & $\mathrm{Cl}$ & $\mathrm{K}$ \\
\hline \hline$u$ & 1 & 12 & 14 & 16 & 23 & 24 & 32 & 35 & 39 \\
\hline$N_{\mathrm{e}^{-}}$ & 1 & 6 & 7 & 8 & 11 & 12 & 16 & 17 & 19 \\
\hline$f_{X}$ & 0.28 & 1.69 & 1.97 & 2.16 & 3.10 & 3.38 & 4.51 & 4.79 & 5.36 \\
\hline
\end{tabular}

\subsubsection{Porod's Law}

Independently developed by Günther Porod in 1951 [84] and Peter Debye et.al. in 1957 [85], Porod's law states that the asymptotic behavior of the final slope of an $I(q)$ profile, corresponding to a sample of monodisperse three-dimensional particles, can be described as:

$$
I(q)=\frac{2 \pi}{q^{4}}(\Delta \rho)^{2} S,
$$

where $S$ is the interface area between the scatterers and environment. If the sample is in vacuum, the scattered amplitude, and thus contrast, is proportional to the electron density $\rho$ of the sample itself. If the sample is not in vacuum, as is the case in the scope of this thesis, the contrast is effectively defined using the difference in electron density between the sample and environment: $\Delta \rho=\rho_{\text {sample }}-\rho_{\text {env }}$. The scattered amplitude is thus proportion to $\Delta \rho$ and the scattered intensity to $(\Delta \rho)^{2}$. In eq. 4.3 , the $q^{-4}$ dependence is valid for systems composed of smooth, 3D objects, as well as for densely packed systems, provided there exists a well-defined internal surface [70]. This fourth-power law can be generalized to

$$
I(q)=K q^{\alpha}+B
$$

where $B$ accounts for inelastic and incoherent scattering, and small density fluctuations within the sample [86]. Note that the units of eq. 4.4 are only well-defined when the exponent $\alpha$ is an integer. The exponent describes the dimensionality and surface roughness of the sample. For smooth, three-dimensional objects $\alpha=-4$, for smooth, two-dimensional objects $\alpha=-2$ and for smooth, one-dimensional objects $\alpha=-1$ [70]. Non-integer values are indicative of samples with a rough surface. Although eq. 4.4 can be used to characterize data when $\alpha \neq-4$, the equation is only referred to as Porod's law when $\alpha=-4$. Consequently, $\alpha$ and $K$ are termed the Porod exponent 
and Porod constant, respectively. The Porod constant is dependent on the electron density contrast and interface area $S$ between the sample and environment, and thus quantitatively describes the aggregation state of the sample:

$$
K=2 \pi(\Delta \rho)^{2} S
$$

It is important to note that eq. 4.5 is only directly applicable for a two-phase system

where $\rho_{\text {sample }} \neq \rho_{\text {env }}$. Thus, it is not directly applicable to biological samples as they are not composed of homogeneous electron density. However, eq. 4.5 does serve as an aid to understand the relationship between $S$ and $\Delta \rho$.

\subsection{Scanning SAXS}

Scanning SAXS was developed in 1995 by Mahendrasingam et. al. [87] at the ID13 beamline of the European Radiation Synchrotron Facility. It was independently developed by Peter Fratzl in 1997 [88] using laboratory sources and was first applied to resolve the size and orientation of particles embedded in bone collagen and cellulose fibrils in wood. It has since been applied to a variety of samples, ranging from teeth [89] and hair [90] to single cells [31, 91-94]. In particular relevance to this thesis, the authors in Ref. [31] used scanning SAXS to study the aggregation and (de-)compaction of chromatin in whole cell nuclei throughout the cell cycle.

The primary difference between SAXS and scanning SAXS is the setup and measurement principle. In SAXS, structural information is accessed in Fourier space by averaging the scattering signal of a large ensemble of randomly oriented scatterers. In comparison, scanning SAXS yields access to information in both Fourier space and real space. In scanning SAXS, as the name implies, the sample is scanned through the X-ray focus spot and a scattering pattern, governed by only the local irradiated structures, is recorded at each scanning location. By scanning the sample it is possible to compute pseudo-real space images via the dark field contrast image. The dark field contrast image is created by summing the number of detected photons per scanning location, thus visualization of the sample is possible by plotting the resulting value in a color-coded fashion at its respective scanning location. As the dark field contrast solely relies on the scattered intensity, it physically represents the overall granularity of the sample. An example of a dark field contrast image is shown in Figure 4.5e. Scanning SAXS is sensitive to structural periodicities. At low $q$ scanning SAXS (and SAXS) is limited by the size of the beamstop, and at high $q$ by the signal level of the largest scattering angle that can be recorded with the detector, which is dependent on the position of the detector. Thus, 
scanning SAXS combines resolution in real space on the order of the beamsize with high resolution in Fourier space, dependent on the available $q$-range.

\subsubsection{Experimental Setup at P10: Scanning SAXS}

All measurements on lyophilized samples presented in this thesis are carried out at the coherence applications beamline P10 at the PETRA III storage ring at DESY (Hamburg, Germany) using the Göttingen Instrument for Nano Imaging with coherent X-rays (GINIX) [95, 96]. The GINIX was specifically designed to image biological cells and tissues using scanning SAXS (section 4.3) and holography (section 4.4), but has now also been extended to tomography (section 4.5) [96, 97]. The experimental configurations of the GINIX in the holography and tomography imaging modalities are discussed in sections 4.4.3 and 4.5.1, respectively. Figure 4.4 shows a simplified schematic of the scanning SAXS setup. An X-ray beam with energy $E=8.0 \mathrm{keV}$ is produced via a $5 \mathrm{~m}$ long undulator and a cryo-cooled double-crystal (Si-111) monochromator. Entrance slits to the Kirkpatrick-Baez (KB) mirrors [98] are tuned to $0.4 \times 0.4 \mathrm{~mm}^{2}$. The Xray beam is focused by the $\mathrm{KB}$ mirrors, oriented in a cross-orthogonal geometry, to a spot size of $350 \times 390 \mathrm{~nm}^{2}$ (vertical $\times$ horizontal, FWHM). The intensity of the beam is approximately $5.5 \times 10^{11}$ photons $\cdot \mathrm{s}^{-1}$ and is cleaned by a set of apertures prior to interacting with the sample. The $\mathrm{Si}_{3} \mathrm{~N}_{4}$ window is mounted on a fully motorized sample tower consisting of three translational motors (SLS-1760 and SHL-1D20N-10, SmarAct, Oldenburg, Germany), and is aligned with an in-line visible light microscope (reflection mode). The X-rays propagate in the $z$ direction and the sample is raster scanned in the $x-y$ plane with step sizes $250 \times 250 \mathrm{~nm}^{2}$. An exposure time of $10 \mathrm{~ms}$ is used. The scattered radiation passes through a vacuum flight tube of $5 \mathrm{~m}$ in length and is recorded on a single photon-counting detector (Eiger 4M, Dectris AG, Baden-Dättwil, Switzerland; $2070 \times 2167$ pixels, pixel size: $75 \times 75 \mu^{2}$ ) placed in the Fraunhofer far-field at a sample-to-detector distance of $5.04 \mathrm{~m}$. A tungsten beamstop (size: $7 \times 8 \mathrm{~mm}^{2}$, thickness: $25 \mu \mathrm{m}$ ) blocks the primary beam from damaging the detector. 


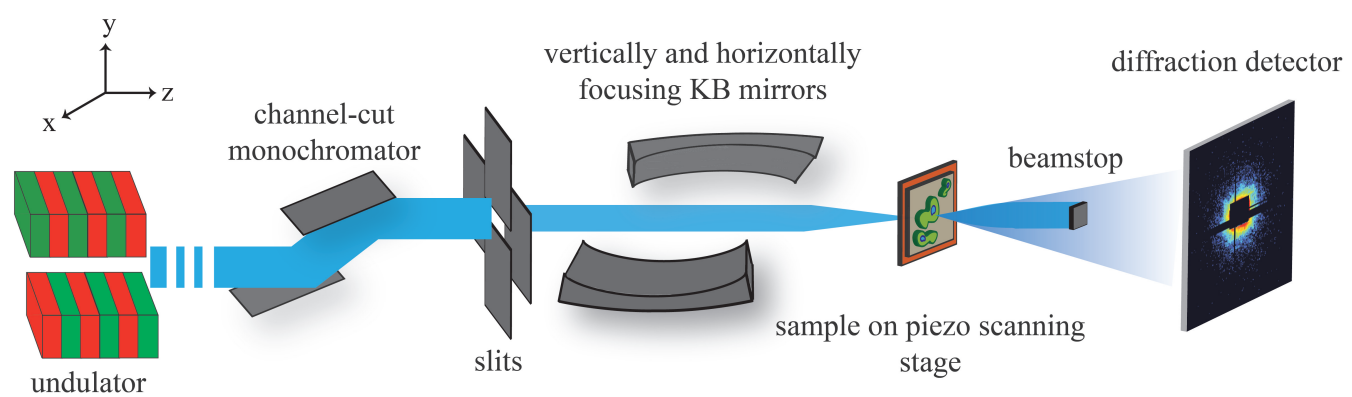

FIgURE 4.4: Schematic of the GINIX setup installed at the coherence application beamline P10 at PETRA III. Here, the GINIX is configured for scanning SAXS. The $\mathrm{X}$-rays are generated in an undulator device and monochromatized by a double-crystal $\mathrm{Si}(111)$ channel-cut monochromator. The X-rays are subsequently cleaned by a pair of slits prior to be focused by a set of Kirkpatrick-Baez (KB) mirrors to a spot size of $350 \times 390 \mathrm{~nm}^{2}$ (vertical $\times$ horizontal, FWHM). The sample is mounted on a piezo sample tower and the sample is raster scanned in the $x-y$ plane. At each scanning point the primary beam is blocked by a beamstop and the scattered intensity is recorded by a diffraction detector.

\subsubsection{Data Analysis: Scanning SAXS}

As shown throughout Figure 4.5, the scanning SAXS data analysis process begins by first summing the scattering patterns acquired at all scanning locations (Figure 4.5b). The resulting pattern is used to define a binary mask. The purpose of the binary mask is to render specific regions of the detected scattering patterns null from subsequent analysis. These regions include the inter-module gap positions of the detector, the beamstop and any malfunctioning pixels. These regions are set to zero in the binary mask (black, Figure 4.5c).

A dark field contrast image is subsequently computed. The term "dark field" comes from the fact that the primary X-ray beam is blocked by the beamstop, thus only the scattered photons are included in the image. Every masked 2D scattering pattern is summed in both the $x$ and $y$ directions to obtain a single value. As the Eiger $4 \mathrm{M}$ detector is a single photon counting detector, the dark field contrast is directly related to the number of detected photons scattered by the sample. The single value obtained from summing each scattering pattern is plotted at the corresponding $x$ and $y$ position of the scan in a color-coded fashion, thus creating the dark field contrast image (Figure 4.5e). Hence, the resolution of the dark field contrast image is limited by the step size between scan points, while the resolution of each scattering pattern is limited by the recorded $q$-range. As can be seen in Figure 4.5e, the dark field contrast shows regions of lower or higher amounts of scattering, enabling the visualization of structures such as the cell body and nucleus. The dark field contrast is thus used to define regions of interest (ROIs) of the sample, e.g., the cell nucleus, cytoplasm and background. Figure 4.5f shows manually selected 
ROIs of the dark field shown in Figure 4.5e. Similar to SAXS data analysis (section 4.2 ), the next step in the presented data analysis is to azimuthally integrate the $2 \mathrm{D}$ scattering patterns. The scattering patterns belonging to each ROI are averaged prior to integration. The resulting $I(q)$ curve is then background corrected by subtracting the averaged $I(q)$ profile belonging to background ROI. The profile is subsequently corrected by the scan exposure time and fitted to the generalized Porod's law (eq. 4.4) to retrieve the $\alpha$ and $K$ coefficients, as described in section 4.2.2. Individual scattering patterns are also analyzed in the same manner. All analysis steps are performed using self-written MATLAB (The MathWorks, Inc., Natick, Massachusetts) scripts or scripts adapted from previous members of the Institute for X-Ray Physics (Göttingen, Germany).

a)

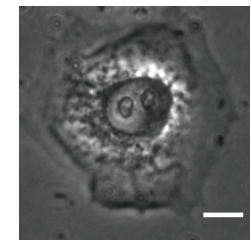

b)

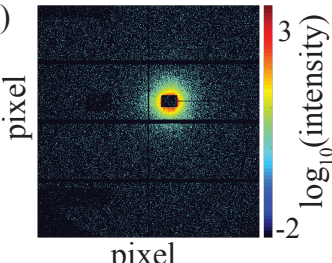

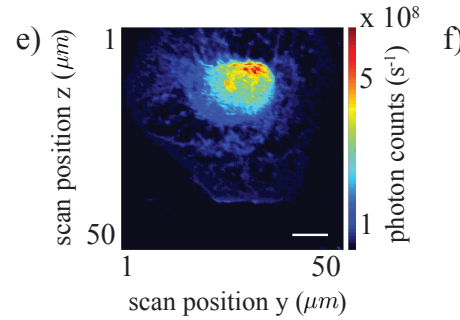

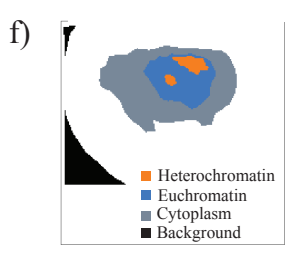

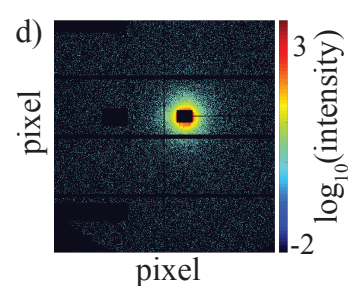

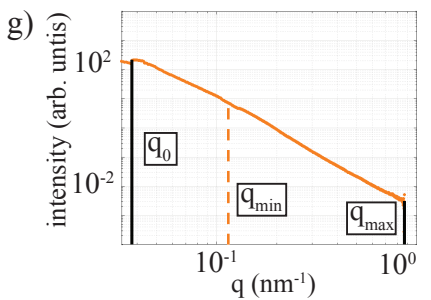

FIGURE 4.5: a) $20 \times$ phase contrast image of a chemically-fixed cell. The scale bar is $10 \mu \mathrm{m}$. b) Sum of all scattering patterns, including those from the sample and background. c) Binary mask to null (black) the beamstop, inter-module gap positions of the detector and malfunction pixels from subsequent analysis. d) Masked scattering pattern shown in b). e) The dark field contrast image of the cell shown in a). The scale bar is $10 \mu \mathrm{m}$. f) Manually selected regions of interest. g) Radial intensity of the averaged heterochromatin signal. The left and right vertical black lines represent $q_{0}$ and $q_{\max }$, respectively. The vertical, dashed orange line represents $q_{\min }$.

Note that in the case of a system such as a whole cell, using Porod's law to fit $I(q)$ requires the analysis to be interpreted with care as the system is much more complex than simple 3D monodisperse particles, for which Porod's theory was initially developed. Towards this end, and recalling that $I(q)=K q^{\alpha}+B$ is only explicitly referred to as Porod's law when $\alpha=-4$, thus enabling the relationship $K=2 \pi(\Delta \rho)^{2} S$ to be valid, an $I(q)$ profile is fitted two separate times: once in the range $\left[q_{\min }, q_{\max }\right]$, where the slope decays as $\alpha=-4$, and again in the range $\left[q_{0}, q_{\mathrm{min}}\right]$, where the slope decays freely. To determine $\left[q_{\min }, q_{\max }\right]$, the averaged $I(q)$ profile belonging to each ROI of every cell is systematically fit with different $q$-ranges using eq. 4.4 and the fit coefficient for the exponent, $\alpha_{\text {fit }}$, is compared to the theoretical value $\alpha_{\text {theory }}=-4$. All $q$ values for which 
$\alpha_{\text {fit }}-\alpha_{\text {theory }}$ is within one standard error are taken into consideration. The largest consecutive $q$-range within the consideration list defines the $\left[q_{\min }, q_{\max }\right]$ range. The momentum transfer $q_{\text {min }}$ defines the point in an $I(q)$ profile where the slope transitions from $\neq-4$ to -4 . The minimum and maximum momentum transfer values incorporated into the scanning SAXS data analysis are defined by $q_{0}$ and $q_{\max }$, respectively. For all scanning SAXS measurements presented in this thesis, $q_{0}=0.038 \mathrm{~nm}^{-1}$ and $q_{\max }=$ $0.867 \mathrm{~nm}^{-1}$. An $I(q)$ profile is thus fitted twice: once in the range $\left[q_{\min }, q_{\max }\right]$, where the Porod exponent $\alpha=-4$ exists, to determine the Porod constant $K$, and a second time in the range $\left[q_{0}, q_{\min }\right]$ to determine the exponent $\alpha$. An example of a fitted $I(q)$ profile is shown in Figure 4.5f. By determining $\alpha$ and $K$, information regarding the morphology and relationship between the square of the electron density contrast and interface area (eq. 4.5) is gained for relevant length scales in the corresponding $q$-range. The $\left[q_{0}, q_{\min }\right]$ and $\left[q_{\min }, q_{\max }\right]$ ranges defined using an averaged $I(q)$ profile from a specific ROI are used when fitting individual scattering patterns associated with the same ROI. Thus, it is possible to represent the data by creating maps of the exponent $\alpha$ and Porod constant $K$, as shown in Figure 4.6a and b, respectively.

a)

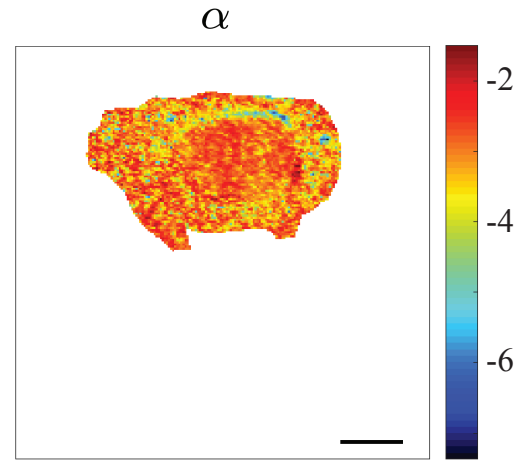

b)

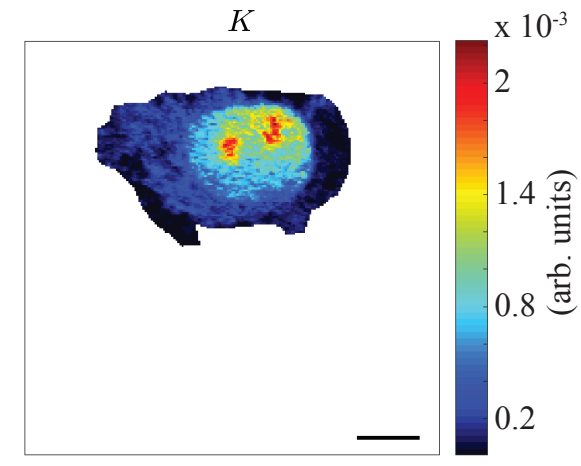

FIgURE 4.6: a) Map of the exponent $\alpha$ values determined by fitting individual $I(q)$ radial intensity profiles within the range $\left[q_{0}, q_{\text {min }}\right]$. b) Map of the Porod constant values determined by fitting individual $I(q)$ radial intensity profiles within the range $\left[q_{\min }, q_{\max }\right]$, where the Porod exponent $\alpha=-4$ exists. Both scale bars are $10 \mu \mathrm{m}$.

\subsection{X-ray Holography}

In scanning SAXS, resolution in real space and Fourier space is combined in a hybrid manner. The method can probe local structures (in Fourier space) in a range smaller than the X-ray beamsize. In comparison, X-ray holography enables direct imaging in real space with resolution below the beamsize. In 1948, Dennis Gabor proposed a method aimed at correcting spherical aberrations in electron microscopy by introducing a lensless imaging technique [15]. Nowadays, this is referred to as in-line holography. The term 
"in-line" indicates the incident and scattered waves share the same optical axis. X-ray in-line holography, hereafter simply referred to as "holography", is a full-field imaging technique where a hologram (Fresnel diffraction pattern) is formed by exploiting the interference pattern between X-rays scattered from a sample and a known reference wave. Encoded in the hologram is the phase and electron density information of the sample [99]. As holography is a $2 \mathrm{D}$ imaging technique, it is sensitive to information projected along the propagation direction of the incident wave field. Thus, information about the, e.g., 3D electron density, is not obtained but rather the 2D projected electron density (electron density per area).

One requirement of holography is that the incident wave field needs to be coherent. To accommodate this, in the presented X-ray study, the X-ray beam is coupled into an Xray waveguide [96] which filters out non-coherent and non-monochromatic photons from the beam. An X-ray waveguide acts as a quasi point-source ( $\leq 20 \mathrm{~nm}[100])$ illumination which produces spherical wave fields. The incorporation of the waveguide increases the numerical aperture of the system and thus enables access to higher real space resolution. By placing the sample in a defocused position away from the waveguide, the magnification and field-of-view (FOV) of the setup can be tailored to accommodate the imaging of either single or multiple cells. Acquired holograms are inverted and reconstructed by phase retrieval algorithms. This allows for the projected electron density distribution $\bar{\rho}_{\mathrm{e}^{-}}(x, y)$ of the sample to be quantified. In the most-simple case, the phase shift of the sample is retrieved by a single inversion using e.g., the contrast transfer function (CTF) $[99,101]$ algorithm. However, in many cases, the strict assumptions about the sample and the incident wave field leads the CTF to introduce artifacts into the reconstruction process [68]. These artifacts can be circumvented by iterative algorithms. For both single and iterative algorithms, the phase reconstruction process can be improved by including measurements at multiple defocused positions [102].

\subsubsection{Direct Reconstruction: Contrast Transfer Function}

The CTF describes how information is transferred as a function of spatial frequency. Biological cells can be considered to have a slowly varying phase [99], i.e., the sample slowly varies over length scales comparable to the wavelength of X-rays. For samples which have a slowly varying phase, the CTF exploits a linear relationship between the Fourier transform of the optical phase and the Fourier transform of the recorded intensity of the holograms.

Recall the waveguide acts as a point source emitting spherical wave fields (Figure 4.7a). The CTF theory assumes a plane wave illumination (Figure 4.7b). To properly exploit 
the CTF, a variable transformation must therefore be considered to assume the sample is illuminated by an effective plane wave. This is done using the Fresnel scaling theorem [103]. It states that the measured intensity distribution of an object illuminated by a point source is equivalent to that of an object illuminated by a plane wave if the sampleto-detector distance $z_{2}$ is converted to an effective propagation distance $z_{\text {eff }}$, and the detector pixel size is shrunk by a magnification factor of $M$. See Figure 4.7.

a) point-source illumination

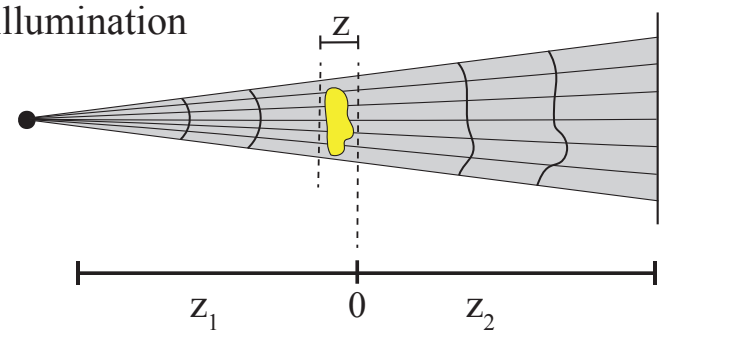

b) $\begin{gathered}\text { plane-wave detector } \\ \text { illumination }\end{gathered}$

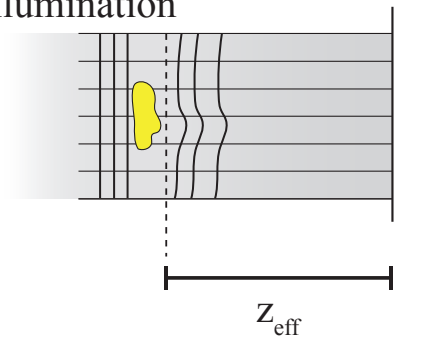

FIGURE 4.7: Schematic representation of the Fresnel scaling theorem. a) An X-ray waveguide acts as a point source emitting spherical wave fields propagating in the $z$ direction. The sample (yellow) of thickness $z$ is placed at a source-to-sample distance $z_{1}$ and interference patterns between the incident and transmitted beam are recorded at a sample-to-detector distance $z_{2}$ with a geometrical magnification of $M=\frac{z_{1}+z_{2}}{z_{1}}$. This can also be described by an b) effective parallel-beam geometry with an effective sample-to-detector distance $z_{e f f}=\frac{z_{2}}{M}$ and effective pixel size $p_{\text {eff }}=\frac{p}{M}$.

For a point source emitting a spherical wave field, the effective propagation distance $z_{\text {eff }}$ is described as [68]:

$$
z_{\text {eff }}:=\frac{z_{1} z_{2}}{z_{1}+z_{2}}=\frac{z_{2}}{M} .
$$

where $M$ is the magnification of the experimental setup:

$$
M=\frac{z_{1}+z_{2}}{z_{1}}
$$

The effective pixel size $p_{\text {eff }}$ is given by:

$$
p_{e f f}=\frac{p}{M}
$$

where $p$ is the detector pixel size. The magnification and effective pixel size are related to the effective Fresnel number $F_{\text {eff: }}$ :

$$
F_{\mathrm{eff}}:=\frac{p_{\mathrm{eff}}{ }^{2}}{\lambda z_{\mathrm{eff}}}=\frac{p^{2}}{M \lambda z_{2}} .
$$


$F_{\text {eff }}$ is a dimensionless quantity which scales with the magnification such that a higher $M$ leads to a smaller $F_{\text {eff }}$. Holograms begin to form when $F_{\text {eff }}<1$.

Consider a monochromatic and coherent plane wave being transmitted through a biological sample of thickness $z$. As X-rays are electromagnetic waves, transmission through the sample not only leads to a decrease in amplitude but also to a phase shift. The strength of the phase shift is determined by the real part $\bar{\delta}(x, y)$ of the projected complex index of refraction, $\bar{n}(x, y)$ :

$$
\bar{n}(x, y)=1-\bar{\delta}(x, y)+i \bar{\beta}(x, y)
$$

while the imaginary decrement $\bar{\beta}(x, y)$ gives rise to the absorption. For biological samples and hard X-rays, $\bar{\delta}(x, y)$ is up to a factor of 1000 larger compared to $\bar{\beta}(x, y)$ [97]. The (projected) decrement $\bar{\delta}(x, y)$ is related to the projected electron density $\bar{\rho}_{\mathrm{e}^{-}}(x, y)$ of the sample $[33,71]$ :

$$
\bar{\delta}(x, y) \approx \frac{r_{0} \lambda^{2}}{2 \pi} \bar{\rho}_{\mathrm{e}^{-}}(x, y),
$$

where $r_{0}$ is the classical electron radius (eq. 3.30) and $\lambda$ is the wavelength of the incident X-rays. A similar relationship between $\bar{\beta}(x, y)$ and $\bar{\rho}_{\mathrm{e}^{-}}(x, y)$ cannot be derived $[68,97]$

The incident wave $u_{\text {inc }}(x, y)$ propagating along the $z$ axis is transmitted by the sample and the change in wave amplitude and phase is described by multiplication of a complex transmission function $T(x, y)$. The transmitted wave $u_{0}(x, y)$ can thus be described as:

$$
u_{0}(x, y)=T(x, y) \cdot u_{\text {inc }}(x, y) \text { with } T(x, y)=e^{-B(x, y)} e^{i \phi(x, y)} .
$$

The subscript " 0 " indicates the position directly after the sample (see Figure 4.7a). The terms $\phi$ and $B$ represent the changes in phase and amplitude of the wave field due to the real and imaginary part of $\bar{n}(x, y)$, respectively. The projected phase of the sample is thus:

$$
\bar{\phi}(x, y)=-\frac{2 \pi}{\lambda} \int_{0}^{z} \delta(x, y, z) d z
$$

and the projected absorption:

$$
\bar{B}(x, y)=\frac{2 \pi}{\lambda} \int_{0}^{z} \beta(x, y, z) d z .
$$

The wave field $u_{z}(x, y)$ at $z_{\text {eff }}$ is described by the convolution with the complex propagator $P$ :

$$
u_{z, \mathrm{eff}}(x, y)=\left(P_{z, \mathrm{eff}} * u_{0}\right)_{x, y}
$$

with

$$
P_{z, \mathrm{eff}}(x, y)=\frac{1}{i \lambda z_{2}} \exp \left(i \pi \frac{x^{2}+y^{2}}{\lambda z_{\mathrm{eff}}}\right)
$$


where $\lambda$ is the wavelength of the incident X-rays. In real space, the intensity is calculated by $I(x, y)=|u(x, y)|^{2}$. In Fourier space, and neglecting the effects of absorption $B$ as $B \ll \phi$, eq. 4.15 is simplified to the CTF $[99,101,104]$ :

$$
\tilde{I}_{z}(f) \approx \delta_{D}(f)+2 \sin \left(\pi \lambda z_{\mathrm{eff}} f^{2}\right) \cdot \tilde{\phi}(f)
$$

where functions with a tilde denote its Fourier transform and $\delta_{D}$ is the Dirac distribution at the spatial frequency $f=0$. Thus, eq. 4.17 gives a relationship in Fourier space between the intensity $\tilde{I}_{z}(f)$ and phase $\tilde{\phi}(f)$. As discussed in section 4.4, the CTF function introduces artifacts into the reconstruction process. This is evident by the $\sin \left(\pi \lambda z_{\text {eff }} f^{2}\right)$ term in eq. 4.17: for certain spatial frequencies $f$, the sine argument will be zero, thus information at these frequencies will not be acquired. To circumvent this, holograms are recorded at multiple defocused positions, thus allowing for the spatial frequencies lost at one defocused position to be acquired in another defocused position. With the phase of the sample known, it is possible to combine eq. 4.11 and eq. 4.13 to determine the projected electron density $\bar{\rho}_{\mathrm{e}^{-}}(\mathbf{r})$ of the sample:

$$
\bar{\rho}_{\mathrm{e}^{-}}(x, y) \approx \frac{-\phi(x, y)}{r_{0} \lambda}
$$

The projected electron density is related to the projected mass density (mass density per area) $\bar{\rho}_{m}(\mathbf{r})$ :

$$
\bar{\rho}_{m}(x, y) \approx\left(\frac{-2 u}{r_{0} \lambda}\right) \cdot \phi(x, y) .
$$

The factor of two is dependent on the chemical composition of the sample. For biological samples with the average empirical formula $\mathrm{H}_{50} \mathrm{C}_{30} \mathrm{~N}_{9} \mathrm{O}_{10} \mathrm{~S}_{1}$, the factor of two valid [33].

\subsubsection{Iterative Reconstruction: Relaxed Averaged Alternating Reflec- tions}

The relaxed averaged alternating reflections (RAAR) algorithm was introduced by Russell Luke in 2004 [105]. RAAR belongs to a class of alternating projection algorithms which iteratively determines the phase of the object by sequentially propagating a wave field $\psi$ between the sample plane $\psi(x, y)$ and detector plane $\psi\left(x^{\prime}, y^{\prime}\right)$. The RAAR algorithm is adapted from an error-reduction (ER) algorithm [68]. Briefly, the ER considers the measured intensity at the detector plane $I\left(x^{\prime}, y^{\prime}\right)$ and incorporates an object constraint, e.g., a support constraint $S$ which accounts for the shape of a spatially resolvable object, such as a biological cell. The aim of the ER is to determine the phase $\phi$ inside of $S$ while simultaneously requiring the phase outside of $S$ to be zero. Thus, the ER systematically evaluates the wave equation $\psi^{(n)}$ to render all $(x, y)$ locations within $S$ 
to be non-zero and all locations outside of $S$ to be zero. The iteration number is represented by $n$. In particular, the reconstruction process is initialized by combining the measured intensity $I$ with a random phase $\phi_{0}(x, y) \in[-\pi, \pi]$ to define the initial wave field $\psi^{(0)}(x, y)$ in the sample plane:

$$
\psi^{(0)}(x, y)=\sqrt{I(x, y)} \cdot \exp \left[i \phi_{0}(x, y)\right]
$$

This wave field is forward propagated to the detector plane using the near-field propagator $D_{z}[68]$ to render the updated wave field $\psi^{(n)}\left(x^{\prime}, y^{\prime}\right)$. The amplitude of this reconstructed wave is replaced with the measured amplitude, which is mathematically equivalent to the square root of the measured intensity. This is done using the magnitude constraint operator $P_{m}[68]$ :

$$
P_{m}[\psi(x, y)]=D_{-z}\left[\sqrt{I\left(x^{\prime}, y^{\prime}\right)} \cdot \frac{D_{z}[\psi]}{\left|D_{z}[\psi]\right|}\right],
$$

to render $\psi^{(n)^{\prime}}\left(x^{\prime}, y^{\prime}\right)$. This wave field is subsequently back-propagated to the sample plane and the object support $P_{s}$ is applied to account for the support constraint $S$ :

$$
P_{s}[\psi(x, y)]= \begin{cases}\psi(x, y) & \forall(x, y) \in S \\ 0 & \text { else }\end{cases}
$$

This iteration process occurs $n$ times until convergence thresholds are met, as detailed in Ref. [68]. The entire iteration process is summarized as:

$$
\psi^{(n+1)}(x, y)=P_{s}\left[P_{m}\left[\psi^{(n)}(x, y)\right]\right]
$$

and is schematically represented in Figure 4.8

Sample plane

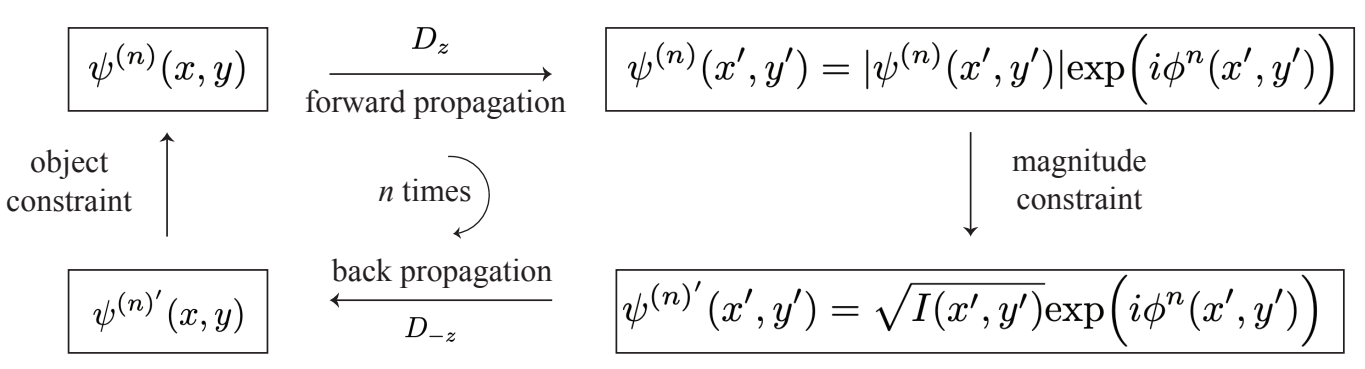

FiguRE 4.8: Schematic representation of the error-reduction algorithm. The wave field $\psi^{(n)}(x, y)$ in the sample plane is forward-propagated to the detector plane to render the wave field $\psi^{(n)}\left(x^{\prime}, y^{\prime}\right)$. The amplitude of this wave field is replaced with the square root of the measured intensity to form $\psi^{(n)^{\prime}}\left(x^{\prime}, y^{\prime}\right)$. This wave field is back-propagated to the sample plane $\left(\psi^{(n)^{\prime}}(x, y)\right)$, where a support constraint is applied to accommodate a spatially resolvable object. 
The RAAR algorithm is a special case of eq. 4.23 which slowly converges $\psi(x, y)$ to zero outside of $S$ using the relation parameter $\beta_{n} \in[0,1]$ :

$$
\psi^{(n+1)}=\left(\frac{\beta_{n}}{2}\left(R_{1} R_{M}+\mathbb{1}\right)+\left(1-\beta_{n}\right) P_{M}\right) \psi^{(n)} .
$$

The identity operator is denoted as $\mathbb{1}$. Both $R_{1}$ and $R_{M}$ are reflectors which take into account the measured intensity $P_{M}$. An in-depth mathematical review of the RAAR algorithm (eq. 4.24) can be found in Ref. [105].

\subsubsection{Experimental Setup at P10: Holography}

The GINIX endstation at P10 has the ability to easily change between the scanning SAXS imaging modality (Figure 4.4) and the holography imaging modality [96], as shown in Figure 4.9. The experimental parameters of the holography measurements are presented in Table 4.2. The KB focused X-ray beam is coupled into an X-ray waveguide consisting of lithography-defined channels in silicon [106]. The waveguide acts as a low-pass filter, suppressing high frequency artifacts which might be produced by sub-nanometer irregularities on the surface of the KB mirrors. The entrance and exit dimensions of the waveguide are $74 \times 70 \mathrm{~nm}^{2}$ and $75 \times 50 \mathrm{~nm}^{2}$, respectively. After coupling into the waveguide, the intensity of the X-ray beam is approximately $2.9 \times 10^{9}$ photons $\cdot \mathrm{s}^{-1}$. The $\mathrm{Si}_{3} \mathrm{~N}_{4}$ window is mounted on a fully motorized sample tower consisting of three translational motors (SLS-1760 and SHL-1D20N-10, SmarAct), allowing to align the sample to the incident wave field with nanometer precision.

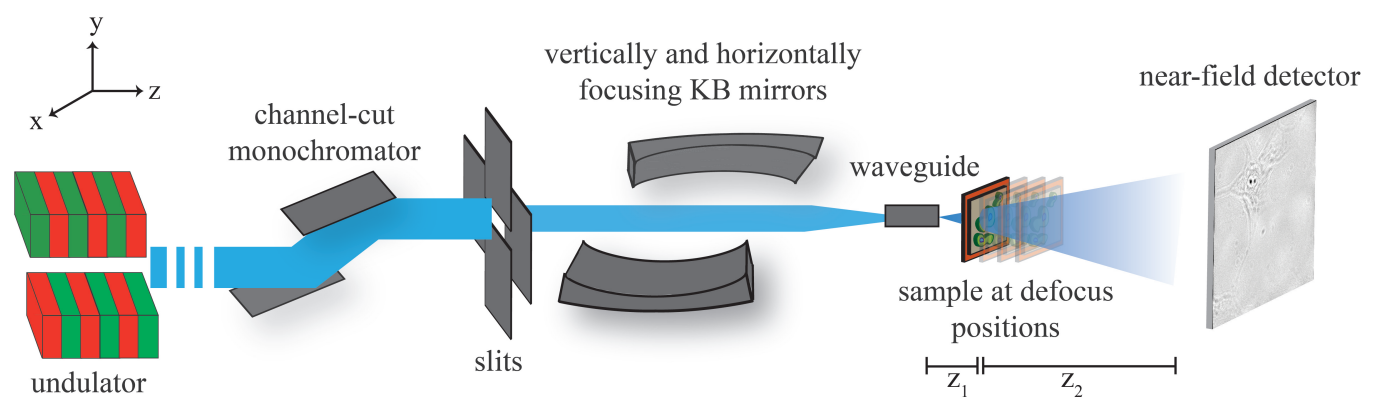

Figure 4.9: Schematic of the GINIX setup installed at the coherence application beamline P10 at PETRA III in the in-line holography configuration. The KB-focused beam is coupled into an X-ray waveguide which acts as a quasi point-source illumination creating a diverging wave field. The sample is placed in the defocused $z_{1}$ and full-field holograms are recorded with a near-field sCMOS imaging camera located at a sampleto-detector distance $z_{2}$.

In the sample plane, individual holograms with a FOV of $33 \times 33 \mu^{2}$ and spatial resolution of $53 \mathrm{~nm}$ are recorded by a scintillator-based fiber-coupled scientific CMOS camera 
(Photonic Science, East Sussex, U.K.; $2048 \times 2048$ pixels, pixel size: $6.5 \times 6.5 \mathrm{\mu m}^{2}$ ). The camera, with the laboratory name "Pirra", is located on the same detector bench as the Eiger $4 \mathrm{M}$ detector used in the scanning SAXS measurements. When imaging multiple cells, the effective FOV is enlarged to $0.33 \times 0.33 \mathrm{~mm}^{2}$ by acquiring holograms in a raster fashion. These holograms are then stitched together in a mosaic fashion. Holographic projections are recorded at four defocused distances of $z_{1}$. Per distance, 50 empty images are acquired. An empty image is the recorded intensity when the sample is not in the beam path and is used when correcting the data. Furthermore, a dark image is acquired which images the noise pattern of the detector. Both the dark and empty images are used for data correction.

TABLE 4.2: Overview of the holography experimental parameters used to measure lyophilized cells adhered to a $\mathrm{Si}_{3} \mathrm{~N}_{4}$ window at P10 (Run 57).

\begin{tabular}{|c|c|}
\hline GINIX run & 57 \\
\hline energy $(\mathrm{keV})$ & 8.0 \\
\hline detector & sCMOS camera \\
\hline number of distances & 4 \\
\hline$z_{1}(\mathrm{~mm})$ & $\{25,27,32,38.8\}$ \\
\hline$z_{2}(\mathrm{~m})$ & $\{5.02,5.02,5.01,5.00\}$ \\
\hline magnification & $\{201.8,186.9,157.7,130.1\}$ \\
\hline$p_{\text {eff }}(\mathrm{nm})$ & $\{32.4,34.9,41.5,50.3\}$ \\
\hline$F_{\text {eff }}\left(1 \times 10^{-3}\right)$ & $\{0.257,0.239,0.204,0.169\}$ \\
\hline number of projections & 10 \\
\hline exposure time $(\mathrm{s})$ & .06 \\
\hline
\end{tabular}

\subsubsection{Data Analysis: X-ray Holography}

The phase retrieval process begins by first background correcting the projections recorded at each defocused distance $i$. Per distance, a projection $I_{\text {proj,i }}$ (Figure 4.10a) is corrected by the averaged dark image $I_{\text {dark,i }}$ (Figure 4.10b) and averaged empty image $I_{\text {empty,i }}$ (Figure 4.10c):

$$
I_{\mathrm{proj}, \mathrm{i}}^{\text {corrected }}=\frac{I_{\text {proj }, \mathrm{i}}-I_{\mathrm{dark}, \mathrm{i}}}{I_{\mathrm{empty}, \mathrm{i}}-I_{\mathrm{dark}, \mathrm{i}}} .
$$

A single corrected projection $I_{\text {proj }, \mathrm{i}}^{\text {corred }}$ belonging to the first defocus position is shown in Figure 4.10d. 

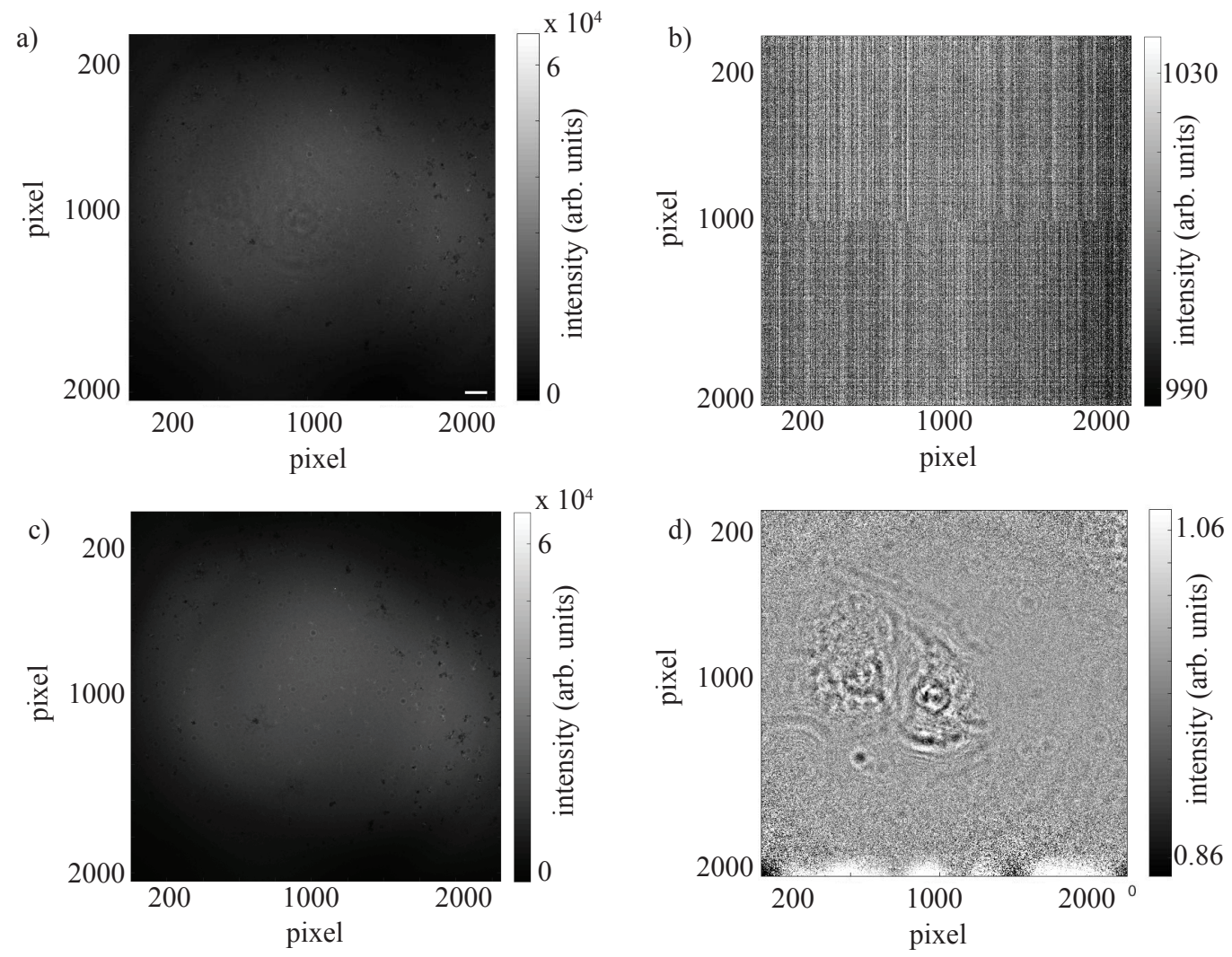

Figure 4.10: a) A (magnified) holographic projection at distance $i$ is recorded by the detector and subsequently background corrected using b) the averaged dark image and c) averaged empty image belonging to $i$ (eq. 4.25). The corrected holographic projection is shown in d). The scale bar in a) is $1 \mathrm{~cm}$ and applies to all subfigures.

To retrieve the holograms used for the phase reconstruction process, the corrected projections recorded at the latter three defocused positions are rescaled to match the magnification of the first defocused position. The rescaled projections are subsequently aligned via a sub-pixel imaging registration algorithm to account for any lateral shifts that occurred during the imaging acquisition process [107]. The rescaled and realigned holograms are shown in Figure 4.11a. In addition to irregularities on the KB mirrors, small positional fluctuations of the focused beam lead to a decrease in stability of the illumination profile. This introduces low frequency artifacts in the holograms. To minimize this effect, a high-pass filter is applied on each corrected hologram. Following this step, the holograms are ready for both direct $(\mathrm{CTF})$ and iterative (RAAR) reconstruction algorithms.

As a first approach, the holograms are numerically processed using the CTF algorithm (eq. 4.17). The resulting reconstructed hologram, i.e., the reconstructed phase map, is shown in Figure 4.11b. This reconstructed phase map was rendered using holograms 
recorded at four defocused positions. As can be seen in the phase map, there are lighter, almost bright, regions, as indicated by the two arrows. Such artifacts stem from the constraints of the low frequencies in the CTF that are necessary to suppress the effects of low frequency background variations. These artifacts are improved upon by use of the iterative RAAR algorithm (eq. 4.24). Towards this end, the reconstructed phase map using the CTF is used to define the support constraint $S$ for the single distance iterative RAAR algorithm (eq. 4.24). The support constrain is represented by the black, dashed line in Figure 4.11b. In total, 500 iteration rounds are performed with the RAAR algorithm, and the relaxation parameter $\beta$ is set to 0.9 . The resulting reconstructed phase map rendered using the RAAR algorithm is shown in Figure 4.11c. At the time of analysis, the implementation of the multiple distance RAAR algorithm was not available. However, as discussed in Ref. [108], the use of single distance iterative reconstruction algorithms yields superior results over the multiple distance CTF algorithm. Furthermore, the single distance iterative reconstruction is only moderately improved when multiple distance are considered. For these reasons, holographic data presented in this thesis was not reanalyzed with the multiple-distance RAAR algorithm.
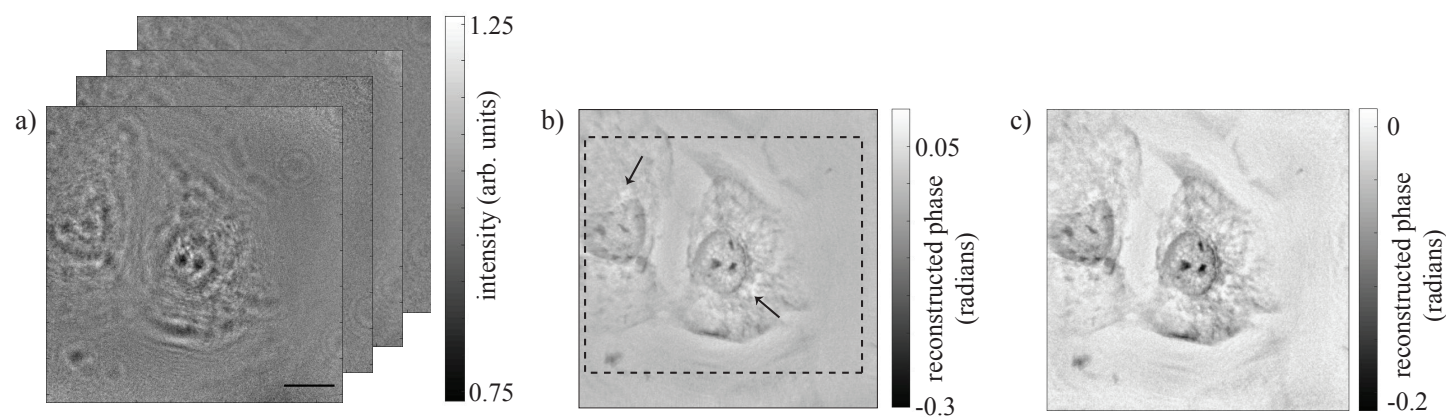

Figure 4.11: a) Averaged and corrected holograms at four defocused positions. The scale bar is $10 \mu \mathrm{m}$ and applies to all subfigures. b) The reconstructed phase map rendered using the CTF algorithm. The dashed, black line represent the support constraint used for the iterative RAAR algorithm. c) Reconstructed phase map rendered using the RAAR algorithm.

Holography is a 2D propagation-based imaging technique that accesses the projected phase shift, and thus projected electron and mass densities, of the sample. To be extended to three dimensions, X-ray computed tomography must be employed.

\subsection{X-ray Computed Tomography}

The general aim of X-ray computed tomography is to reconstruct a $n$-dimensional object from a series of different projections, each recorded at a different angle. More precisely, 
the spatial distribution of an observable $f$ is reconstructed in $n$-dimensional space from projections acquired in $n-1$ sub-spaces [68]. The observable $f$ can represent e.g., the real decrement $\delta$ (eq. 4.11) and therefore the electron density of the sample. The necessary mathematical framework was developed by Johann Radon in 1917 [109] and is briefly introduced here, following Ref. [68]. For a detailed mathematical description of X-ray tomography, readers are referred to Refs. [68, 97, 110, 111].

Let $(x, y, z)$ describe the coordinate system of the laboratory frame, comprising the source and detector, and $\left(x^{\prime}, y^{\prime}, z^{\prime}\right)$ describe the object. The rotation angle of the sample about the mutual $y^{\prime}=y$ axis is described by the rotation angle $\theta \in\left[0,180^{\circ}\right)$. To obtain the structure of a $3 \mathrm{D}$ sample, given by $f$, projections of the sample have to be recorded under different rotation angles. The set of projection images are recorded by rotating the object in a fixed X-ray beam propagating in the $z$ direction. The function $f_{y}\left(x^{\prime}, z^{\prime}\right)$ describes the sample at a constant height $y$, therefore depicting one $2 \mathrm{D}$ slice through the $3 \mathrm{D}$ volume. The projection $P_{y}(\theta, x)$ of the function $f_{y}\left(x^{\prime}, z^{\prime}\right)$ at an angle $\theta$ onto the detector is given by:

$$
\begin{aligned}
P_{y}(\theta, x)=R f(x) & =\int_{\mathbb{R}} f_{y}\left(x^{\prime}, z^{\prime}\right) \mathrm{d} z \\
& =\int_{\mathbb{R}} f(x \cos \theta-z \sin \theta, x \cos \theta+z \cos \theta) \mathrm{d} z,
\end{aligned}
$$

where $R$ denotes the 2D Radon transform operator [68, 109]. This relationship is schematically represented in Figure 4.12 , where the object $f_{y}\left(x^{\prime}, z^{\prime}\right)$ is illuminated at the rotation angle $\theta$, leading to the projection $P_{y}(\theta, x)$ (red curve). 


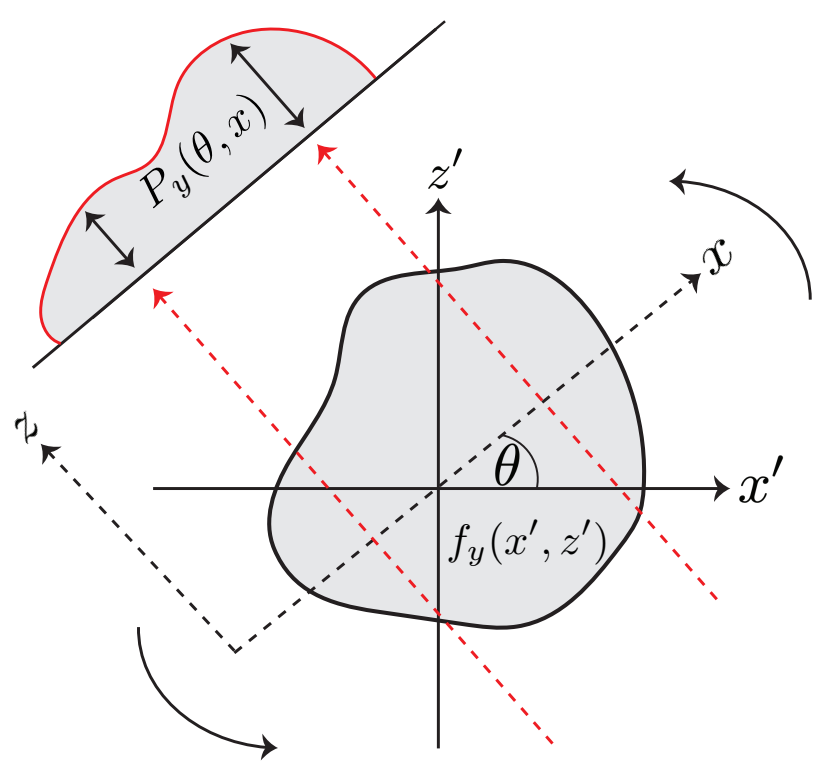

Figure 4.12: At a constant height $y$ an object is described by the function $f_{y}\left(x^{\prime}, z^{\prime}\right)$. The object is illuminated at a given rotation angle $\theta$, leading to the projection $P_{y}(\theta, x)$.

Equation 4.27 can be alternatively be expressed as [112]:

$$
R f(x)=\int_{\mathbb{R}^{2}} f\left(x^{\prime}, z^{\prime}\right) \delta_{D}\left(x^{\prime} \cos \theta+z \sin \theta-x\right) \mathrm{d} x^{\prime} \mathrm{d} z^{\prime},
$$

where $\delta_{D}$ is the Dirac delta distribution. Hence, the Radon transform is performed by integrating over straight lines along $z$ with distance $x$ to the origin (represented by the red dashed lines in Figure 4.12). For a single point located at $\left(x_{0}^{\prime}, z_{0}^{\prime}\right)$ within the sample, with $f\left(x^{\prime}, z^{\prime}\right)=\delta_{D}\left(x^{\prime}-x_{0}^{\prime}\right) \delta_{D}\left(z-z_{0}^{\prime}\right)$, the Radon transform is written as:

$$
R f(x)=\delta_{D}(x-r \sin (\theta-\alpha)),
$$

where $r=\sqrt{x_{0}^{\prime 2}+y_{0}^{\prime 2}}$ and $\alpha=\operatorname{atan}\left(x_{0}^{\prime} / y_{0}^{\prime}\right)$. Hence, the Radon transform of a single point is represented by a sinusoidal curve. As an object can be regarded as a combination of single points, the Radon transform is a superposition of sine curves. The plot of projections $P_{y}(\theta, x)$ against the angle $\theta$ is called a sinogram. Figure 4.13b shows the sinogram corresponding to the three squares shown in Figure 4.13a. The dashed, red lines represent projections $P_{y}(\theta, x)$ acquired at $\theta=45^{\circ}$ and $\theta=135^{\circ}$.

To obtain the phase or amplitude of the original 2D function $f_{y}\left(x^{\prime}, z^{\prime}\right)$, the inverse Radon transform operator $R^{-1}$ is required. Its application yields:

$$
f_{y}\left(x^{\prime}, z^{\prime}\right)=R^{-1}\left[P_{y}(\theta, x)\right]
$$


for a plane of constant $y$. By evaluating several planes at different $y$ (Figure 4.13c), the $3 \mathrm{D}$ structure $f\left(x^{\prime}, y^{\prime}, z^{\prime}\right)$ of the sample can be obtained (Figure 4.13d).

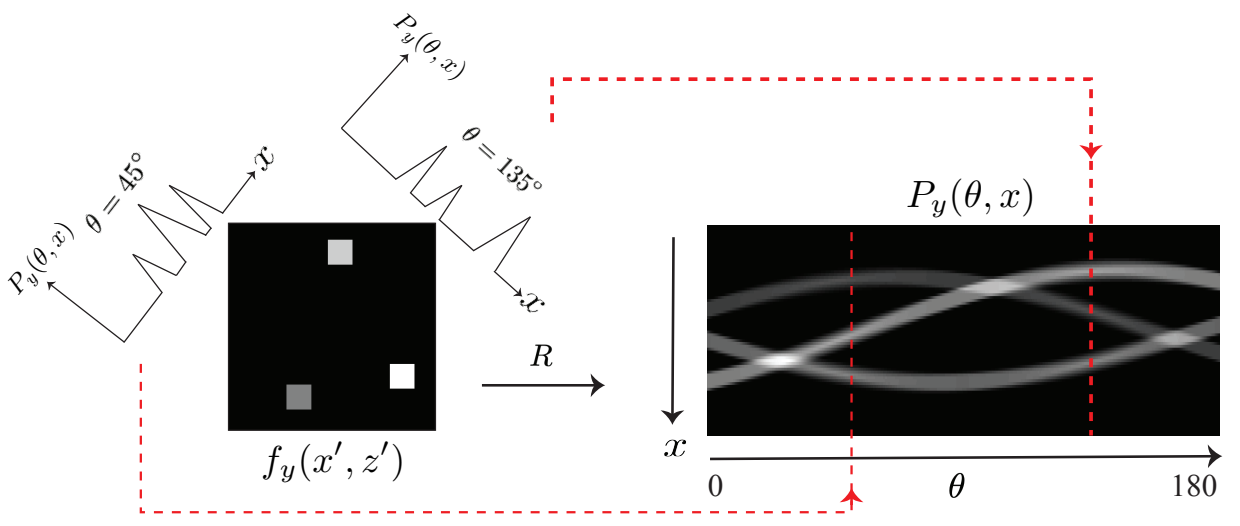

a)

d) b)

c)

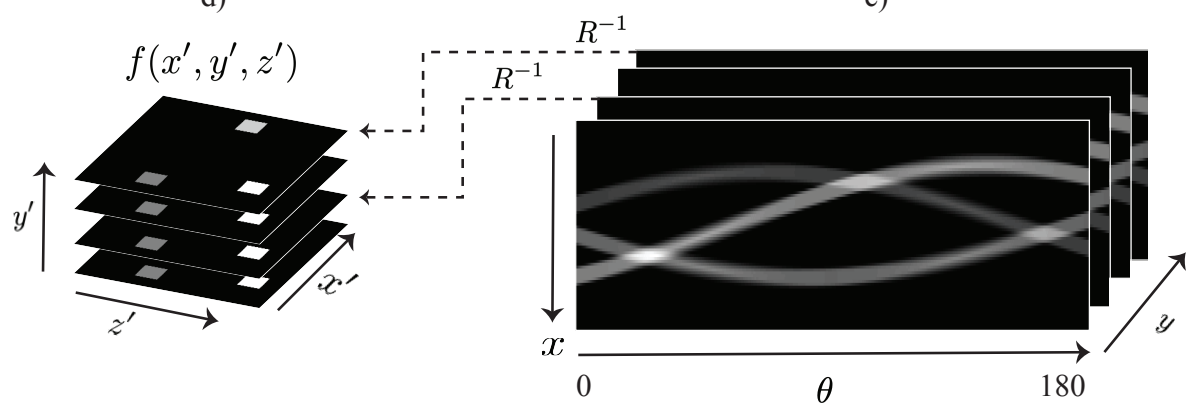

Figure 4.13: a) A sample is described by the function $f_{y}\left(x^{\prime}, z^{\prime}\right)$. Projections $P_{y}(\theta, x)$ corresponding to rotation angles $\theta=45^{\circ}$ and $\theta=135^{\circ}$ are shown. For each parallel plane along the rotation axis $y=y^{\prime}$, a set of projections is recorded for a given angular range. b) All projections $P_{y}(\theta, x)$ of the sample plotted against $\theta$ leads to the sinogram. The dashed, red lines represent the projections $P_{y}(\theta, x)$ acquired at $\theta=45^{\circ}$ and $\theta=135^{\circ}$. c) From a $y$-stack of sinograms $P_{y}(\theta, x)$, the d) 3D function $f\left(x^{\prime}, y^{\prime}, z^{\prime}\right)$ is reconstructed by a series of inverse Radon transforms $R^{-1}$, e.g., the filtered back projection. Figure adapted from Ref. [113].

There are various techniques to invert the Radon transform $[68,97]$ including, for example, the filtered back projection [110, 114]. It can briefly be described as Fourier-filtering the projections $P_{y}(\theta, x)$ with a ramp filter and then back-projecting the filtered projection values along straight lines equal to the paths of the line integrals of the Radon transform. An in-depth mathematical description can be found in Refs. [68, 111].

\subsubsection{Experimental Setup at P10: Tomography}

Although the GINIX was originally designed to image biological samples using scanning SAXS and holography, it has been extended to accommodate tomographic imaging [68, 97], as schematically shown in Figure 4.14. 


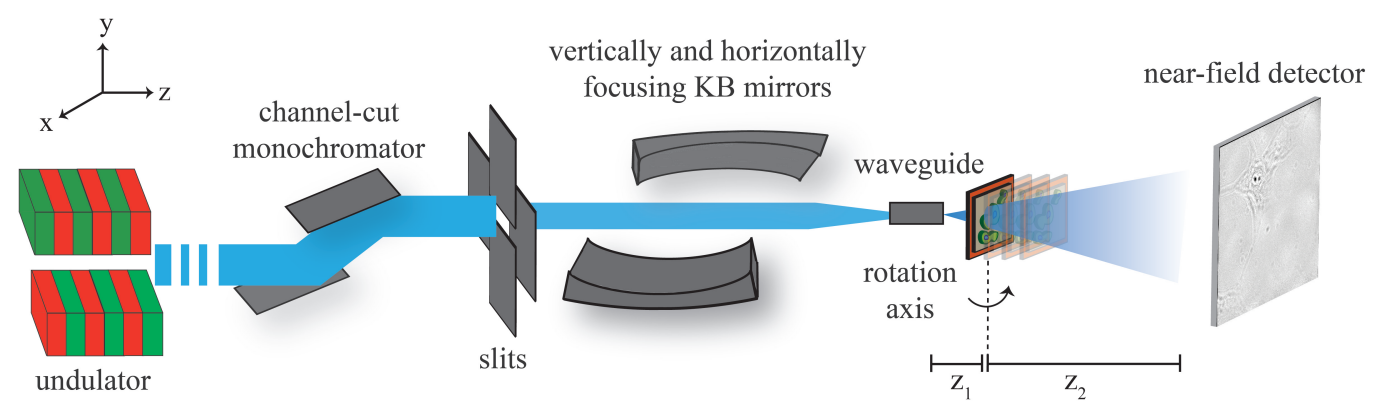

Figure 4.14: Schematic of the GINIX setup installed at the P10 beamline at PETRA III at DESY. In the tomographic imaging modality, the KB-focused beam is coupled into an X-ray waveguide which produces spherical wave fields. The $\mathrm{Si}_{3} \mathrm{~N}_{4}$ window is placed at a series of defocused positions $z_{1}$ and holographic images are recorded at a sample-to-detector distance $z_{2}$. At each defocused position the sample is rotated $180^{\circ}$ and holograms are recorded at each rotation angle.

The experimental parameters of the tomography measurements used to measure both lyophilized and chemically-fixed hydrated samples are presented in Table 4.3. A X-ray beam is produced via a $5 \mathrm{~m}$ long undulator and a cryo-cooled double-crystal (Si-111) monochromator. Entrance slits to the KB mirrors are tuned to $0.4 \times 0.4 \mathrm{~mm}^{2}$. The beam is subsequently coupled into the waveguide. At the exit of the waveguide, the intensity of the beam is approximately $2.1 \times 10^{9}$ photons $\cdot \mathrm{s}^{-1}$. The $\mathrm{Si}_{3} \mathrm{~N}_{4}$ window is mounted on a fully motorized sample tower consisting of three translational motors (SLS-1760 and SHL-1D20N-10, SmarAct) and placed at a series of defocused positions $z_{1}$. Hologram are recorded at a sample-to-detector distances $z_{2}$. Aligning the sample with respect to the rotational axis is performed by correlating projections acquired at $\theta=0^{\circ}$ and $\theta=180^{\circ}$. Once aligned, holograms are recorded over an angular range of $\theta$ by a sCMOS camera. At each defocused position, 20 dark images and 50 empty images are recorded. 
TABLE 4.3: Overview of the holographic tomography experimental parameters used to measure lyophilized cells adhered to a $\mathrm{Si}_{3} \mathrm{~N}_{4}$ and chemically-fixed hydrated cells encapsulated in a polypropylene chamber. Measurements are performed at the P10 beamline of DESY.

\begin{tabular}{|c|c|c|}
\hline Sample & Lyophilized & Chemically-fixed hydrated \\
\hline \hline GINIX run & 79 & 79 \\
\hline energy $(\mathrm{keV})$ & 8.0 & 8.0 \\
\hline detector & sCMOS camera & sCMOS camera \\
\hline number of distances & 4 & 51 \\
\hline$z_{1}(\mathrm{~mm})$ & $\{51,51,55,61\}$ & 5.02 \\
\hline$z_{2}(\mathrm{~m})$ & $\{5.02,5.02,5.02,5.01\}$ & 99.8 \\
\hline magnification & $\{99.8,97.9,92.6,83.4\}$ & 65.1 \\
\hline$p_{\text {eff }}(\mathrm{nm})$ & $\{65.1,66.4,70.2,77.9\}$ & 0.543 \\
\hline$F_{\text {eff }}\left(1 \times 10^{-3}\right)$ & $\{0.543,0.533,0.504,0.455\}$ & {$[0,180]$} \\
\hline angular range $\left(^{\circ}\right)$ & {$[0,180]$} & 1500 \\
\hline number of projections & 1500 & 2 \\
\hline exposure time $(\mathrm{s})$ & 1 & \\
\hline
\end{tabular}

\subsubsection{Data Analysis: X-ray Tomography}

The first step in the tomography analysis is to correct the holograms for each of the 4 distances following eq. 4.25. The corrected holograms recorded at the latter 3 distances are rescaled to match the magnification of the first. The rescaled holograms are subsequently aligned via a sub-pixel imaging registration algorithm [107] to correct for any lateral displacement of the sample during the image acquisition process. Low-frequency background components are removed via a high-pass Gaussian filter. Following this step, the corrected holograms are reconstructed using the CTF algorithm (section 4.4.1). A reconstructed hologram of a lyophilized cell is shown in Figure 4.15a.

Recall the cells are adhered to a $\mathrm{Si}_{3} \mathrm{~N}_{4}$ window and holograms are recorded over an angular range of $\theta=\left[0,180^{\circ}\right]$. At some rotation angles the window edge is parallel to the incident X-rays. Thus, no holograms containing the cell are acquired. This angular range is referred to as the "missing wedge" and projections belonging to it are neglected in subsequent analysis. The missing wedge is represented by the black, vertical region in the sinogram shown in Figure 4.15e. Within the scope of this thesis, the missing wedge varies between $18^{\circ}$ and $49^{\circ}$. The angular range of the missing wedge is dependent on the location of the imaged cell, i.e., how close the cell is to the center axis of the substrate, and the size of the substrate itself. 
Throughout the tomography reconstruction process it is assumed the axis of rotation is located in the vertical center line of the detection plane. However, in practice this is not always the case. If there is a misaligned rotation axis in the experimental data, it is corrected by shifting all recorded projections by the shift distance $d$ between the actual tomographic axis and central line of the detector. As the projection at $\theta=180^{\circ}$ should be, in principle, identical to the projection at $\theta=0^{\circ}$ just flipped around the vertical axis, $d$ is empirically determined such that the difference between the two projections is minimized. An exaggerated example of a misaligned rotation axis is shown in Figure 4.15b. Furthermore, it is possible that the rotation axis can be tilted, i.e., the rotation axis is not positioned along the vertical center line of the detection plane but is tilted by an angle $\phi$. An example of an exaggerated tilt angle is shown in Figure 4.15c. Similar to the shift value $d$, the tilt angle $\phi$ is empirically determined such that the $\theta=0^{\circ}$ and (horizontally flipped) $\theta=180^{\circ}$ reconstructed projections are centered. Applying optimal $d$ and $\phi$ values yields Figure $4.15 \mathrm{~d}$. 
a)

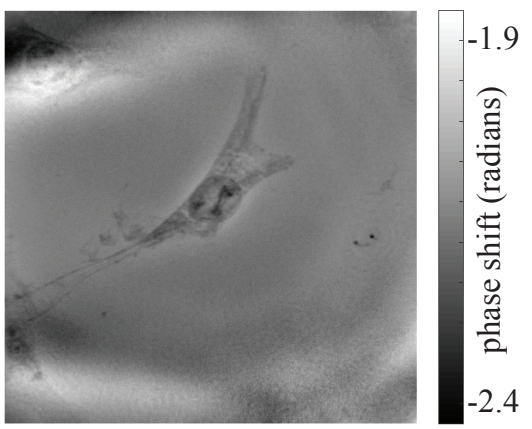

c)

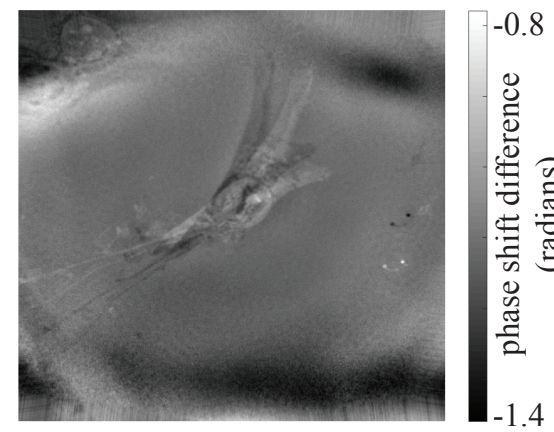

b)

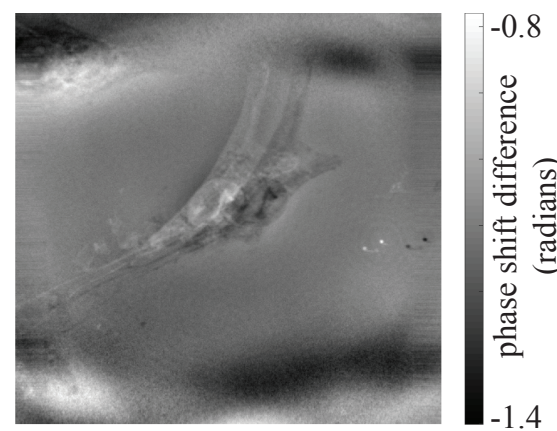

d)

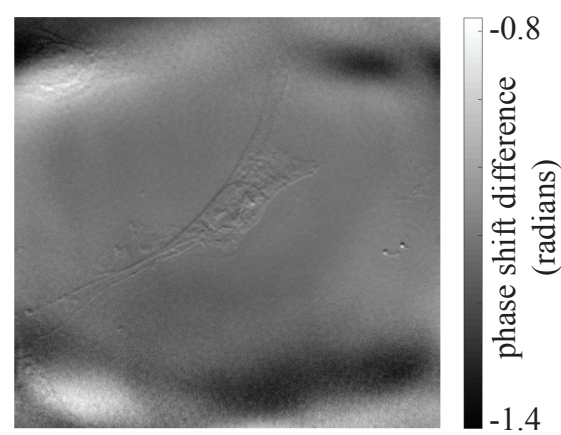

e)

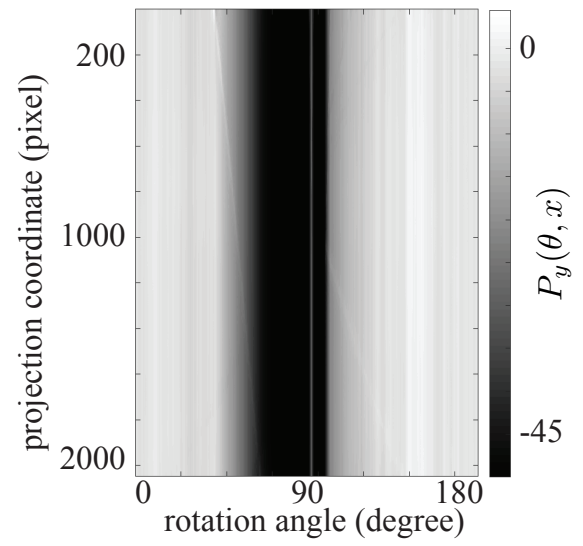

FIGURE 4.15: Steps within the tomography reconstruction process. a) A reconstructed hologram using the CTF algorithm. The effects of an b) severely misaligned rotation axis and c) severely titled rotation axis. d) Difference between the $\theta=0^{\circ}$ and (flipped) $\theta=180^{\circ}$ projections after correcting the rotation axis displacements. e) Sinogram corresponding to the reconstructed hologram in a).

Following the corrections of the rotational axis, every hologram is background corrected by subtracting its mean background signal. The filtered back-projection is then applied using the inverse Radon transform to construct the 3D structure. 


\subsection{A Beamline-Compatible STED Microscope}

Imaging biological samples with X-rays enables them to be visualized in both 2D and 3D. Data interpretation, in particular for scanning SAXS and holography, can be challenging as the 2D signal originates from the entire $3 \mathrm{D}$ ensemble of biomolecular constituents within the sample. Consequently, it can be challenging to directly correlate contributions of the detected signal to a specific structure. As discussed earlier, fluorescent microscopy enables the visualization of specifically labeled biomolecules, thus allowing the investigation of solely those entities. To combine the fluorescent and X-ray imaging modalities, a custom built, beam-line compatible STED microscope is designed and implemented into the GINIX endstation at P10 (DESY, Hamburg, Germany).

In a conventional STED microscope, the laser and optical components are housed in a singular body, to which the inverted microscope body is attached. An oil or water immersion objective with high numerical aperture $(\mathrm{NA}>1)$ is used. Such a setup cannot be coupled into a beamline endstation for two primary reasons: the large size of the unit cannot be accommodated by the X-ray optics table, and an oil or water objective would require the optical axis of the STED microscope to be perpendicular to the propagation axis of the X-rays. As a first proof-of-principle to combine in situ visible-light fluorescence and X-ray microscopy at the same synchrotron endstation, a STED microscope is designed and built to be compatible with the GINIX endstation at P10. In this way, visible-light fluorescence can be combined with scanning SAXS, holography and tomography to visualize both labeled and unlabeled structures in whole cells in a complementary manner. The design and implementation is realized by the collaboration between Abberior Instruments (Göttingen, Germany) and the Institute for X-Ray Physics (University of Göttingen. Göttingen, Germany). In particular, Tim Salditt and Marten Bernhardt of the Institute for X-Ray Physics played a critical role in the process. The custom STED microscope is introduced here, and detailed descriptions can be found in Refs. [93, 115].

To accommodate the physical limitations of the X-ray optics table, the STED microscope is assembled from two separate boxes: The laser and detector box houses the excitation laser $\left(\lambda_{\text {exc }}=632 \mathrm{~nm}\right)$, STED laser $\left(\lambda_{\text {depletion }}=775 \mathrm{~nm}\right)$, avalanche photodiode $(\mathrm{APD})$ and the control computer. The optics box, which can be placed on the X-ray optics table, houses the remaining optical components, including the focusing mirrors and objective. A safety cap integrated to the top of the optics box is part of an inter-lock system. The system prohibits the production of any fluorescent light unless the objective is encased by the cap, thus shielding the surrounding environment. The optics box houses a set of wave plates which exploit polarization, referred to an "easySTED" unit [116]. The unit is comprised of an achromatic quarter-wave plate, a chromatic segmented wave plate and 
an achromatic half-wave plate. Collectively, they render the depletion intensity profile to have zero intensity in the central annulus and the pulsed excitation laser to be circularly polarized, thus resembling a normally-focused spot. The excitation and depletion pulses are separated by 738 ps. The optics box is schematically shown in Figure 4.16.

Front view

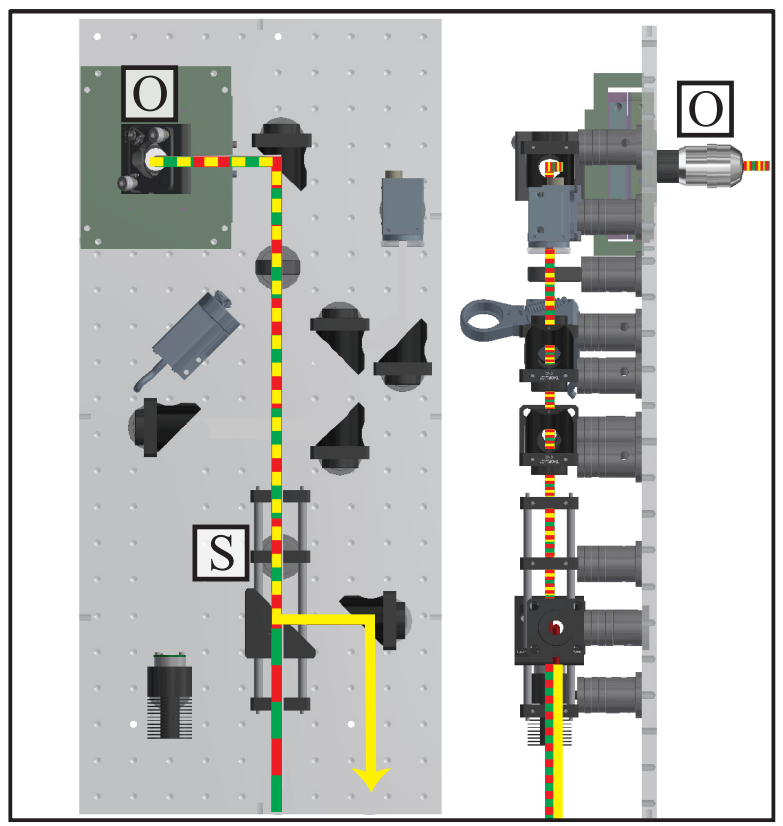

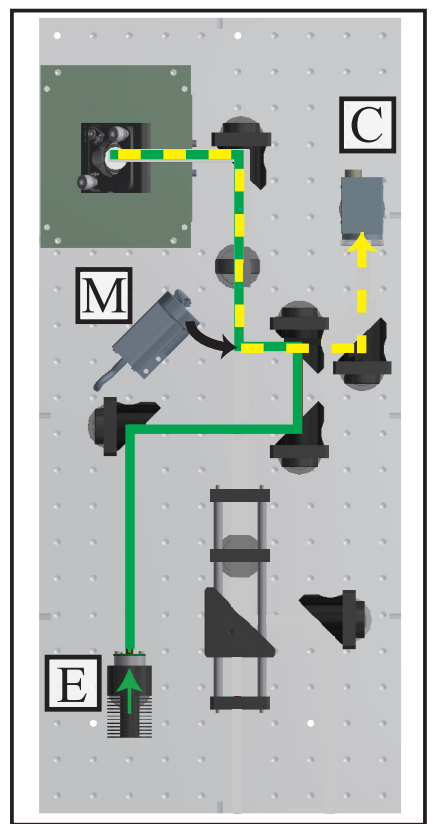

Figure 4.16: The optics box houses all of the focusing optics. Left: In the confocal and STED mode (scanning mode), the excitation laser (green) and depletion laser (red) pass through the "easySTED" unit $(\mathrm{S})$ prior to being focused into the objective (O) The fluorescent single is shown in yellow. Right: In epifluorescence (full-field) mode, a motorized mirror $(\mathrm{M})$ guides the internal epifluorescence laser (green, $(\mathrm{E})$ ) to the objective. The fluorescent signal is detected by a CCD camera (C).

As can be seen in Figure 4.16, the optical axis of the objective is parallel to the ground, thus an oil or water immersion objective is not used but rather a high NA air objective $(40 \times, \mathrm{NA}=0.95$, UPLSAPO, Olympus). However, as discussed in Ref. [115], a possible upgrade of the optics box includes mounting it in a rotatable assembly. This would convert the instrument into an inverted microscope, thus enabling the use of a water or oil immersion objective when used as a stand-alone microscope.

The microscope has two different imaging modes: scanning and full-field. The scanning mode is applied for confocal and STED microscopy, utilizing components in the laser and detector box. The full-field mode is available in either bright-field illumination or epifluorescence and utilizes optical component installed in the optics box. For a typical STED recording, the sample is either a (lyophilized) $\mathrm{Si}_{3} \mathrm{~N}_{4}$ window glued on a brass pin or a (hydrated) sample enclosed in a polypropylene chamber. The sample is mounted on 
a hexapod nano-position system and first inspected in bright-field mode to orientate and navigate the sample. A region of interest (ROI) of the sample is then selected. When a region of interest is found, the sample is coarsely aligned to the focal plane of the objective. The epifluorescence mode is enabled to assess the quality of the fluorescence signal. The sample is then coarsely raster scanned (typical step size $=250 \mathrm{~nm}$ ) in confocal mode in the lateral $x-y$ plane. To finely adjust the sample in the focal plane, line scans are performed in the $x-z$ plane, where $z$ is the direction of the STED beam axis. The sample is subsequently repositioned along $z$ such that it is in the center of the focal plane. Afterwards, a combined confocal and STED scan is recorded in the $x-y$ plane with a typical step size of $80 \mathrm{~nm}$ and dwell time of $0.29 \mathrm{~ms}$. If desired, the sample can also be scanned along the optical axis $z$ to record a 3D fluorescent stack. The fluorescent signal is recorded with respect to the scan position by a single-photoncounting diode.

The optics box and laser and detector box are separated by optical fibers $10 \mathrm{~m}$ in length. When implemented at the beam line endstation, the optics box is placed on the X-ray optics table and the laser and detector box is placed near the X-ray detector. This is schematically represented in Figure 4.17

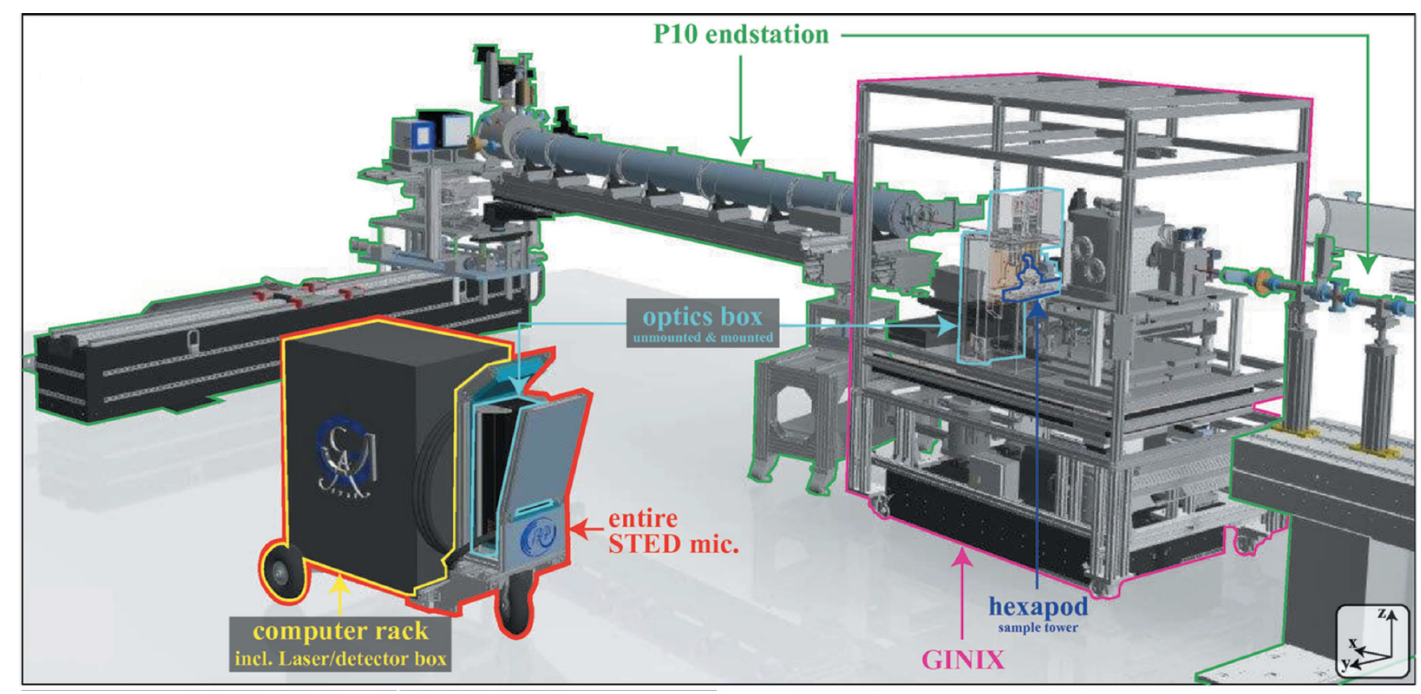

FIgURE 4.17: Schematic of the STED microscope implemented at the GINIX endstation of the P10 beamline at DESY. The laser and detector box (yellow) is placed away from the optics box (cyan), located on the X-ray optics table of the GINIX (pink). This figure is taken from Ref. [115] and is reproduced with permission of the International Union of Crystallography.

To accommodate both the visible-light and X-ray imaging modalities, the hexapod stage is laterally translated $30 \mathrm{~cm}$ from the STED microscope to the X-ray beam path. The 
optical axis of the STED beam is positioned anti-parallel to the X-ray beam. Thus, the sample is also rotated $180^{\circ}$ about the $y$ axis. The lateral stage is show in Figure 4.18.
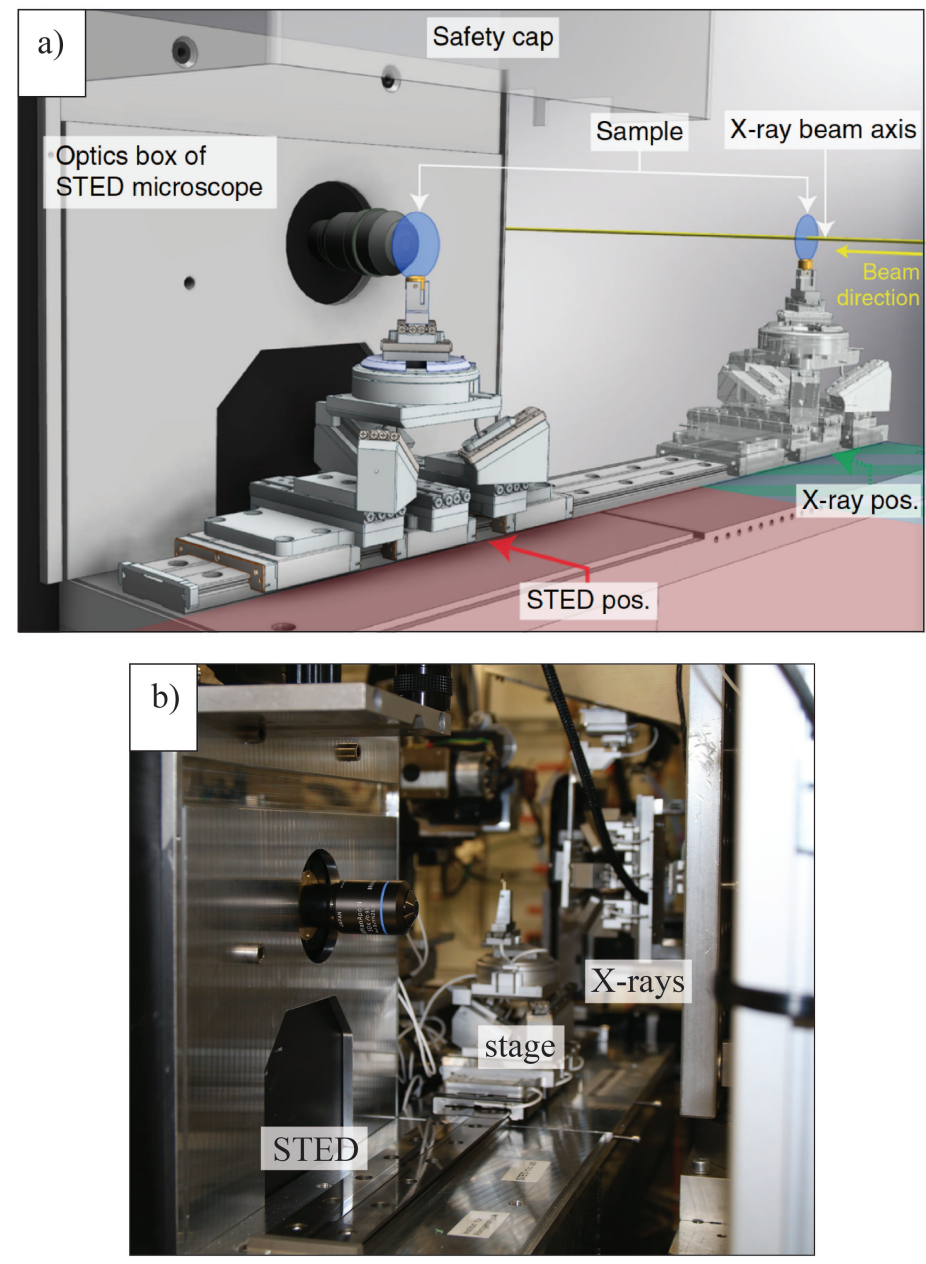

Figure 4.18: a) Schematic of the lateral stage between the STED microscope and $\mathrm{X}$-ray beam path. The sample is translated a distance of $30 \mathrm{~cm}$. This figure is reproduced from Ref. [93]. b) Photography of the lateral stage implemented at the GINIX endstation at P10.

In the following chapter, the results of combining X-ray in-line holography and scanning SAXS to probe the hierarchical structure of DNA are presented. Chapter 6 discusses the results of combining X-ray tomography and visible-light fluorescence to probe structural parameters of DNA. 


\section{Combined Scanning Small Angle X-ray Scattering and Holography Probes Multiple Length Scales in Cell Nuclei}

This chapter has been prepared for publication with authors: Andrew Wittmeier, Chiara Cassini, Mareike Töpperwien, Manuela Denz, Johannes Hagemann, Markus Osterhoff, Tim Salditt and Sarah Köster. The data was collected by A.W., M.T., M.D., M.O., T.S. and S.K.. Analysis was performed by A.W., with input from C.C., M.T. and J.H.. The manuscript was written by A.W. and S.K., with input from all authors. The revised and published version of this chapter is found in Ref. [117]. The corresponding digital object identifier is: 10.1107/S1600577520016276.

\subsection{Introduction}

DNA within mammalian cell nuclei stores genetic information and is densely packed. Indeed, about $2 \mathrm{~m}$ of DNA [9] are found in the nucleus of each human cell within a diameter of roughly $10 \mu \mathrm{m}$. The packing hierarchy spans length scales from $2 \mathrm{~nm}$ to $1 \mu \mathrm{m}$. Techniques to image structures on these length scales primarily use three types of probes, i.e., electrons [118, 119], visible-light fluorescence [120, 121] and X-rays $[122,123]$. Electron microscopy (EM) has the highest spatial resolution but is very invasive, typically requiring the sample to be sliced and stained. Using EM, purified nucleofilaments with a diameter of $10 \mathrm{~nm}$, which fold into higher-order fibers with a $30 \mathrm{~nm}$ diameter, were resolved [5], and it was shown that these fibers subsequently coil into a zig-zag ribbon structure [8]. 
Specific labeling of cellular components as is employed in fluorescence microscopy provides a straight-forward way of identifying exactly these labeled components. Using a combination of fluorescence microscopy and electron tomography, the in situ 3D packing of chromatin in human mitotic chromosomes was described as a disordered and flexible granular chain [42]. With the invention of super-resolution fluorescence techniques [124], e.g., stimulated emission depletion (STED) microscopy [55], stochastic optical reconstruction microscopy (STORM) [125], fluorescence-lifetime imaging microscopy (FLIM) [126] or Förster resonance energy transfer (FRET) microscopy [127], resolution of tens of nanometers can be achieved [124, 125]. Employing these innovative methods, small loops of DNA within mitotic chromosomes have been imaged [40] by STED microscopy and, using STORM, the structure of chromatin fiber was visualized via imaging the histone protein H2B [128]. Within HeLa cells, the in situ interaction of the heterochromatin protein HP1 $\alpha$ and DNA was imaged using a combination of FLIM and FRET microscopy [129].

The strength of fluorescence microscopy is that cellular components are specifically labeled. However, as a consequence, only labeled structures can be imaged. By contrast, $\mathrm{X}$-rays probe electron density directly and thus do not rely on labeling or staining. Moreover, the high penetration depth and small wavelength of X-rays allows us to image thick samples, like whole cells, with resolutions below $100 \mathrm{~nm}$. Scanning small-angle X-ray scattering (SAXS) [88], in particular, combines information from real space and Fourier space. The method was introduced in 1997 and was first used to resolve the size and orientation of particles embedded in bone collagen and cellulose fibrils in wood. Scanning SAXS was also successfully used to study teeth [89], hair [90] and muscle tissue [130]. More recently, scanning SAXS was applied to single cells [91] and, of particular interest for this present study, cell nuclei [31], where the aggregation and (de-)compaction of chromatin was followed throughout the cell cycle.

Another technique to obtain nanometer resolution utilizing X-rays is holography, a fullfield propagation based near-field imaging approach. Quantitative phase contrast imaging is performed and the projected electron density, and thereby mass density, of the sample is investigated. Examples of successful application to single cells are Bacillus thuringiensis [131] and Deinococcus radiodurans [34, 35], where resolutions of $100 \mathrm{~nm}$, $53 \mathrm{~nm}$ and $125 \mathrm{~nm}$, respectively, were obtained. The latter two studies are particularly interesting in the context of the present work as they focus on densely packed DNA in Deinococcus radiodurans.

Joining scanning SAXS and X-ray holography in the same experiment combines the strengths of both methods. Scanning SAXS probes structural and morphological information whereas X-ray holography provides quantitative electron and mass density. 
Combining these methods the authors in Ref. [108] were able to probe the orientation of actomyosin filaments within lyophilized neo-natal rat muscle cells and structural information spanning three orders of magnitude. This work was extended by correlating the X-ray holography and scanning SAXS data with STED images [93].

Here, we apply the very successful combination of scanning SAXS and X-ray holography to nuclei of mammalian cells, a biological system for which the hierarchical combination of different length scales fundamentally defines function. We extend the approach presented by the authors of Refs. [108] and [93] by carefully and quantitatively analyzing four different physical quantities accessible by the combination of scanning SAXS and X-ray holography, namely the relevant length scales, morphology, aggregation and mass or electron density of the scatters, in a spatially resolved manner. We show that only by combining all of this information we are able to identify and localize important nuclear structures, i.e., nucleoli, heterochromatin and euchromatin, thus highlighting the relevance of combined imaging, and characterize the structures according to size, aggregation and density. Thus, we present the results of a label-free technique that is widely applicable to biological samples and can spatially distinguish scattering biological matter across various length scales.

\subsection{Materials and Methods}

NIH-3T3 fibroblasts derived from Swiss albino mouse embryos [82] were cultured in cell culture flasks (Nunc A/S, Roskilde, Denmark) with a $25 \mathrm{~cm}^{2}$ area using high-glucose $\left(4.5 \mathrm{~g} \cdot \mathrm{mL}^{-1}\right)$ Dulbecco's Modified Eagle's Medium (Sigma-Aldrich, Taufkirchen, Germany) supplemented with 10\% (v/v) FBS (Sigma) and 1\% (v/v) penicillin-streptomycin. The cells were stored in a cell incubator kept at $37^{\circ} \mathrm{C}$ and $5 \% \mathrm{CO}_{2}$. Once the cells reached a confluency of $\sim 80 \%$ they were detached from the flask via trypsin incubation $\left(37^{\circ} \mathrm{C}, 5 \%\right.$ $\mathrm{CO}_{2}$ ) for $150 \mathrm{~s}$ using $0.05 \%$ trypsin derived from porcine pancreas (Sigma-Aldrich). A silicon-rich nitride membrane $\left(\mathrm{Si}_{3} \mathrm{~N}_{4}\right.$, membrane size and thickness: $1.5 \times 1.5 \mathrm{~mm}^{2}, 1 \mu \mathrm{m}$, frame size and thickness: $5.0 \times 5.0 \mathrm{~mm}^{2}, 200 \mu \mathrm{m}$; Silson Ltd., Warwickshire, UK), which was previously plasma cleaned using a radio frequency power of $18 \mathrm{~W}$ for $30 \mathrm{~s}$ (Harrick Plasma, PDC-32G, Ithaca, New York, USA), was placed in a $3 \mathrm{~cm}$ diameter Petri dish, where $1.7 \mathrm{~mL}$ of medium and $300 \mu \mathrm{L}$ of cell suspension, with approximately $3.8 \times 10^{5}$ cells - $\mathrm{mL}^{-1}$, were subsequently added. The Petri dish was then placed into the incubator for roughly 48 hours to promote cell adhesion to the $\mathrm{Si}_{3} \mathrm{~N}_{4}$ membrane. The cells were chemically fixed using $3.7 \%$ formaldehyde, stabilized with $1 \%$ methanol, and were then rinsed with $(1 \times)$ phosphate buffered saline (Sigma-Aldrich). After the chemical fixation 
process the cells were vitrified by rapidly plunging them from a water-saturated environment (humidity $\geq 95 \%, 19^{\circ} \mathrm{C}$ ) into a liquid ethane/propane bath at $-196{ }^{\circ} \mathrm{C}$ using a Leica grid plunger (Leica EM GP, Leica Microsystems, Wetzlar, Germany). The cells were stored in liquid nitrogen until the lyophilization process began, where they were transferred into a home-built evacuated chamber which was kept in cryogenic conditions. The temperature of the lyophilization process was gradually increased from $-186^{\circ} \mathrm{C}$ to $15^{\circ} \mathrm{C}$. The cells were kept inside the chamber, with a pressure on the order of $0.01 \mathrm{~Pa}$, for several days in order to ensure the sublimation of any amorphous ice formed during the plunging process. To prevent rehydration, the cells were kept in an evacuated desiccator until they were mounted on the sample stage at the beamline. An inverted light microscope (IX81, Olympus, Hamburg, Germany) was used to record phase contrast micrographs $(20 \times$ objective) immediately before and after the plunging and lyophilization steps; these images were used to monitor the sample preparation process.

\subsubsection{Experimental Setups}

All measurements detailed in this work were performed using the Göttingen Instrument for Nano-Imaging with X-rays (GINIX) [95, 96] setup at the coherence applications beamline P10 at the PETRA III storage ring (DESY, Hamburg, Germany). The beam was delivered via a $5 \mathrm{~m}$ long undulator and subsequently passed through a Si-111 doublecrystal monochromator at an energy of $8.0 \mathrm{keV}$. Entrance slits to the KirkpatrickBaez (KB) mirrors [98] were tuned to $0.4 \times 0.4 \mathrm{~mm}^{2}$. The $\mathrm{KB}$ mirrors, oriented in a cross-orthogonal manner, focused the beam to a spot size of $350 \times 390 \mathrm{~nm}^{2}$ (vertical $\times$ horizontal, FWHM). The beam had an intensity of approximately $5.5 \times 10^{11}$ photons . $\mathrm{s}^{-1}$ and was cleaned by apertures prior to interacting with the sample, which was mounted on a piezo-electric stage capable of lateral movement with nanometer precision. An on-axis visible light microscope, operated in reflectivity mode, was used to locate a desired scanning region of the sample. Scanning SAXS measurements were performed using a scanning step size of $250 \times 250 \mathrm{~nm}^{2}$ and a $10 \mathrm{~ms}$ exposure time. Behind the sample, the X-rays propagated through a $5 \mathrm{~m}$ long evacuated flight tube where the primary beam was blocked by a tungsten beamstop (size: $7 \times 8 \mathrm{~mm}^{2}$, thickness: $25 \mu \mathrm{m}$ ). The scattering signal was recorded using a single-photon counting detector (Eiger $4 \mathrm{M}$, Dectris AG, Baden-Dättwil, Switzerland; $2070 \times 2167$ pixels, pixel size: $75 \times 75 \mathrm{\mu m}^{2}$ ). The effective pixel size of the scanning SAXS measurements was equal to the step size, and the field-of-view was $50 \times 50 \mathrm{\mu m}^{2}$. A schematic of the scanning SAXS setup is shown in Figure 5.1a. 
An attractive feature of the GINIX setup is its ability to change between the scanning SAXS and in-line holography imaging modalities with only minor hardware reconfigurations [96]. To this end, the sample was (initially) placed $25 \mathrm{~mm}$ downstream from the KB focal plane and the focused beam was coupled into an X-ray waveguide consisting of lithography-defined channels in silicon [106]. The waveguide not only coherently filtered the beam but also acted as a quasi point-source $(\leq 20 \mathrm{~nm})$, producing spherical wavefronts. After coupling into the waveguide, the transmitted intensity of the beam was approximately $2.9 \times 10^{9}$ photons $\cdot \mathrm{s}^{-1}$. In the sample plane individual holograms had a field-of-view (FOV) of $33 \times 33 \mu^{2}$ and were recorded by a scientific CMOS $(\mathrm{SCMOS})$ camera (Photonic Science, East Sussex, UK; $2048 \times 2048$ pixels, pixel size: $6.5 \times 6.5 \mathrm{\mu m}^{2}$ ) located on the same detector bench as the Eiger $4 \mathrm{M}$ detector. Multiple holograms could be imaged in a mosaic fashion to accommodate a larger FOV. Holograms were acquired at distances of $X_{1}=\{24.9,26.9,31.8,38.5\} \mathrm{mm}$, with respect to the focus position at $X_{0}=0 \mathrm{~mm}$, with corresponding magnifications and effective pixel sizes of $\{201.8,186.9,165.7,130\}$ and $\{32.4,34.9,41.5,50.3\} \mathrm{nm}$, respectively. Per distance, 10 images were acquired with an exposure time of $60 \mathrm{~ms}$ each. Additionally, 50 empty images were taken per distance. An empty image is the recorded intensity when the sample is not in the beam path. A schematic of the holographic imaging setup is shown in Figure 5.1b.

The radiation dose differs between the two imaging modalities by several orders of magnitude. The dose is estimated from the intensity $I_{0}$ and energy $E_{p h}$ of the incident beam, as well as the exposure time $\tau$ and irradiated area $\Delta_{y} \Delta_{z}$ :

$$
D=\frac{I_{0} \tau E_{p h}}{d \rho_{m} \Delta_{y} \Delta_{z}}
$$

For calculating the dose on a biological sample with the empirical formula $\mathrm{H}_{50} \mathrm{C}_{30} \mathrm{~N}_{9} \mathrm{O}_{10} \mathrm{~S}_{1}$, an attenuation length of $d=7.5 \times 10^{-4} \mathrm{~m}$ and mass density of $\rho_{m}=1.35 \mathrm{~g} \cdot \mathrm{cm}^{-3}$ are commonly used $[74,132]$. Substituting the appropriate values into eq. 5.1 , a dose of $1.1 \times 10^{8}$ Gy and $8.6 \times 10^{3}$ Gy was calculated for the scanning SAXS and X-ray holography measurements, respectively. Note: for the holography calculation, the value of $\tau$ represents the accumulation of the individual exposure times at each of the four defocused position. Furthermore, an additional $50 \mathrm{~ms}$ was considered per distance to account for the time required to open and close the beam shutter. In order to preserve the sample's structural integrity as well as possible, holography measurements were performed prior to the more invasive scanning SAXS measurements. 


\subsubsection{Data Analysis}

The recorded intensities of the scanning SAXS and X-ray holography measurements correspond to the (Fraunhofer) far-field and (Fresnel) near-field, respectively. For the scanning SAXS measurements, to first visualize the cell in a pseudo real space representation, every $2 \mathrm{D}$ scattering pattern was multiplied by a logic mask which rendered unwanted regions (beamstop, flight tube, dead pixels) null from subsequent analysis. By integrating the remaining number of scattered photons of each scattering pattern and plotting the resulting value in a color-coded fashion at its corresponding scanning location, we obtain an X-ray dark field image. An example of a dark field image is shown in Figure 5.2a and cellular and background regions on interest (ROIs) were manually defined (Figure 5.4a). Averaged and individual scattering patterns belonging to each ROI were investigated throughout this work. Full 2D scattering patterns were azimuthally integrated and plotted against the magnitude of the momentum transfer wave vector $q$ to obtain $1 \mathrm{D}$ radial intensity profiles $I(q)$. The values of $q$ are related to the the scattering angle $\theta$ and X-ray wavelength $\lambda[69,70,84]$ via:

$$
q=\frac{4 \pi}{\lambda} \sin (\theta)
$$

This relationship is schematically shown in Figure 5.1a. The experimental setup yielded access to a $q$-range of $\left[q_{0}, q_{\max }\right]=[0.038,0.867] \mathrm{nm}^{-1}$, corresponding to a range of $[7,157] \mathrm{nm}$ in real space. The radial intensity profiles $I(q)$ were background-corrected, normalized by the exposure time and fitted with a power law (eq. 5.3) using a non-linear least-squares minimization. The fitting procedure was performed twice: once between $q_{0}$ and $q_{\min }$ to determine the exponent $\alpha$, and a second time between $q_{\min }$ and $q_{\max }$ to determine $K$. The momentum transfer $q_{\min }$ denotes the point in an $I(q)$ profile where the slope transitions from $\neq-4$ to -4 . The $\left[q_{\min }, q_{\max }\right]$ range was uniquely determined for each of the ROIs of every individual cell using the following criteria: Every possible $q$-range of an averaged $I(q)$ profile was fitted using eq. 5.3 and we chose the threshold to require the difference between the fit coefficient for $\alpha$ and the theoretical value $\alpha=-4$ to be less than one standard error. The largest $q$-range which satisfied the threshold was subsequently used to define $\left[q_{\min }, q_{\max }\right]$ for the particular ROI of the individual cell. This $q$-range defined the fitting range used when fitting individual $I(q)$ profiles to determine the Porod constant $K$. 


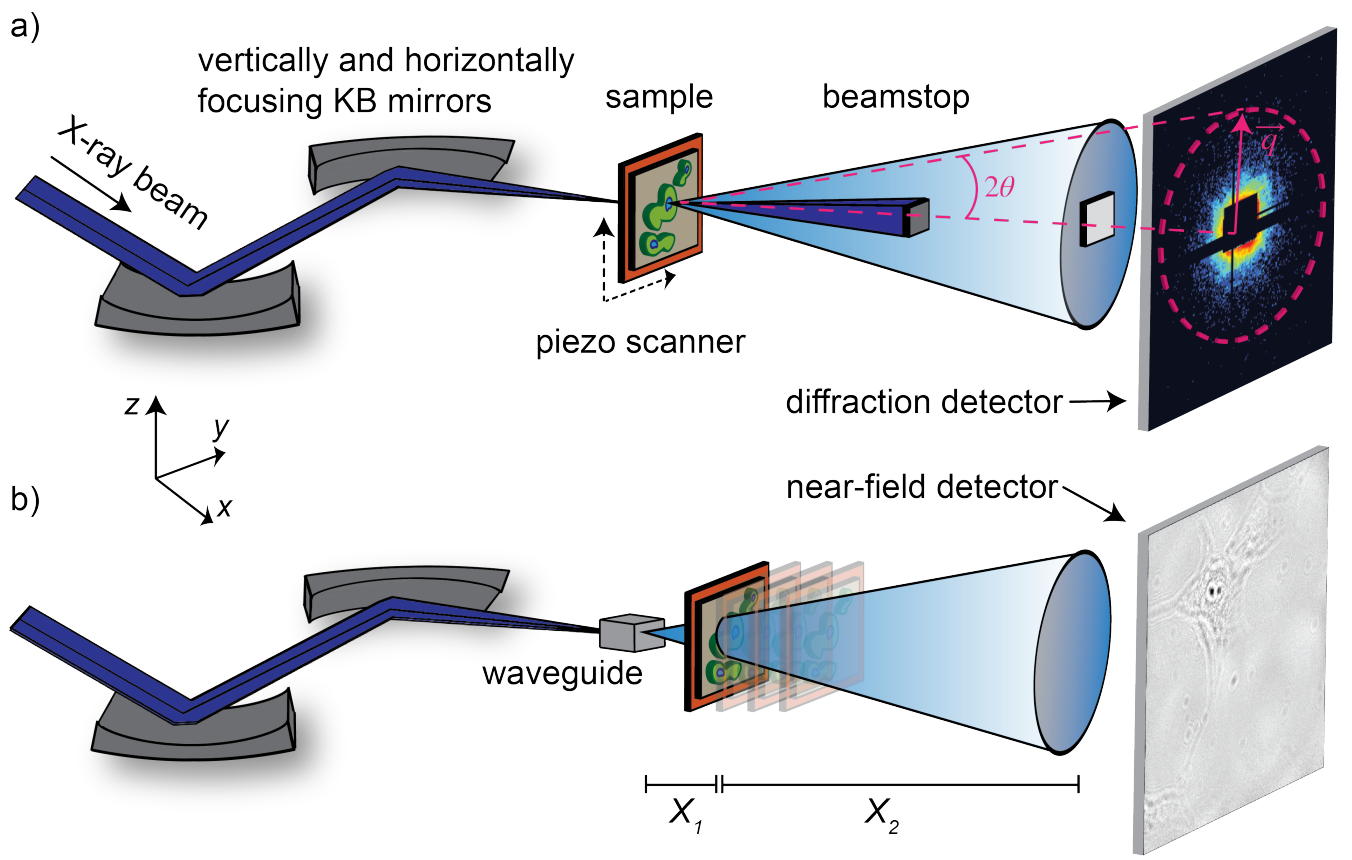

Figure 5.1: Schematics of the setups used for (a) scanning SAXS and (b) in-line holography using the GINIX setup at the P10 coherence application beamline at the PETRA III storage ring, DESY, Hamburg [95, 96]. (a) The X-rays are focused by a set of Kirkpatrick-Baez (KB) mirrors prior to being scattered from the sample. The sample is raster scanned in the $y-z$ plane and at each scanning position a scattering pattern is recorded by a single-photon counting pixelated detector. The dashed purple lines illustrate the relationship between the scattering angle $2 \theta$ and the momentum transfer vector $\vec{q}$. (b) The KB-focused beam is coupled into a waveguide which acts as a quasi point-source creating a diverging wavefront. The sample is placed at a series of defocused positions $X_{1}$ and full-field holograms are recorded at each distance with a sCMOS imaging camera located at a distance $X_{2}$ behind the sample.

Concerning the holography measurements, the acquired holograms of each distance were first averaged and background corrected by division by their respective averaged empty image. The background corrected holograms were subsequently rescaled to match the magnification of those recorded at the first defocused position and were then aligned via a sub-pixel image registration algorithm [107] to account for any lateral shifts that occurred during the image acquisition process. Following these steps, the in-line holograms were ready for both direct and iterative reconstruction algorithms. To this end, the holograms were first numerically processed using the contrast transfer function (CFT) algorithm [99]. The resulting 2D reconstructed phase map was used to define the support constraint for the single distance iterative relaxed averaged alternating reflections (RAAR) [105] algorithm. In total, 500 iteration rounds were performed to render the reconstructed phase map observed in Figure 5.2d. The reconstructed phase contrast, $\phi(x, y)=\phi_{\text {sample }}-\phi_{\text {bg }}$, was used to quantify both the projected mass density (eq. 5.6) and projected electron density (eq. 5.5). 
The FOV and effective pixel sizes rendered from the holography and scanning SAXS measurements differ. In order to correlate a reconstructed phase map, and subsequently a projected mass or electron density map, to those derived from the SAXS measurements in a pixel-wise manner, image registration must be performed. To this end, a reconstructed phase map was registered to a dark field image via a self-written MATLAB (The MathWorks, Inc., Natick, MA, USA) script. The reconstructed phase map was first resized such that it had the same effective pixel size as that of the dark field. The cpselect tool was then used to manually select several anchor points. Any prominent characteristics that were clearly distinguishable in both images were suitable candidates for anchors. On average, 7 anchors were chosen per cell. The reconstructed phase map then underwent the necessary rotational and translational transforms such that its anchor points had the same spatial coordinates as those in the dark field.

\subsection{Results and Discussion}

\subsubsection{Aggregation State of DNA in Cell Nuclei}

All measurements presented in this work are performed on lyophilized NIH-3T3 fibroblasts cultured on X-ray transmissive silicon-nitride substrates. Although in general the lyophilization process may alter nanostructures and thus damage the sample, [133] found the integrity of mammalian DNA remains intact directly after the process. For scanning SAXS measurements, the sample is placed in the focal plane of the X-ray beam and is subsequently raster scanned, as depicted in Figure 5.1a. At each scanning position a scattering pattern, determined by the size, morphology and electron density of the scatterers, is recorded. For every cell investigated here, a total of $201 \times 201=40401$ scattering patterns are recorded and used to render dark field images. The dark field contrast provides an integrated quantity that does not distinguish between the length scales covered by the recorded data range. A dark field is a pseudo real space representation of a sample and physically represents its overall granularity. An example for such a dark field image calculated using the entire usable data range $\left[q_{0}, q_{\max }\right]=[0.038,0.867]$ $\mathrm{nm}^{-1}$, corresponding to length scales in the $[7,157] \mathrm{nm}$ range in real space, of a cell in interphase is shown in Figure 5.2a. Visible light micrographs of the cell in the chemically fixed and lyophilized state are shown in Figure 5.6.

The dark field image shows an integrated quantity and reveals how much the electron density of the sample differs from the background. As it does not distinguish between length scales, to access the relevant scales within different regions of the nucleus we 
calculate dark field images for different $q$-ranges, as shown in Figure 5.3. In this representation, the color scales of the individual dark field images differ, and account for the minimum and maximum of the total number of detected photons within the corresponding $q$-range. A version of Figure 5.3, where all dark field images have the same color scale adjusted to the minimum and maximum number of photon counts found throughout all 6 images, is shown in Figure 5.7. By calculating various dark field images, we are able to spatially distinguish areas which contain structures of different Fourier components, corresponding to certain real space ranges. These ranges are chosen to correspond to the relevant length scales of the formation of chromatin and its subsequent packing.

a)

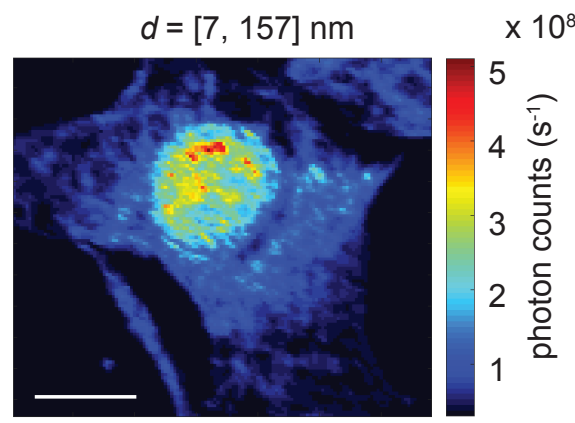

c)

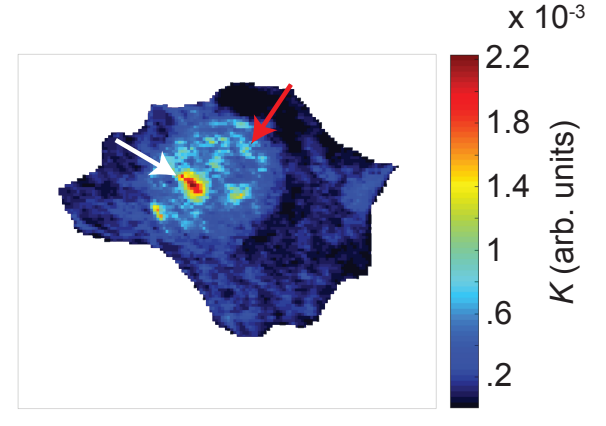

b)

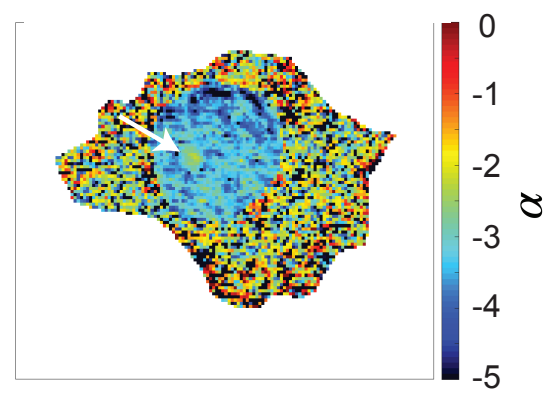

d)

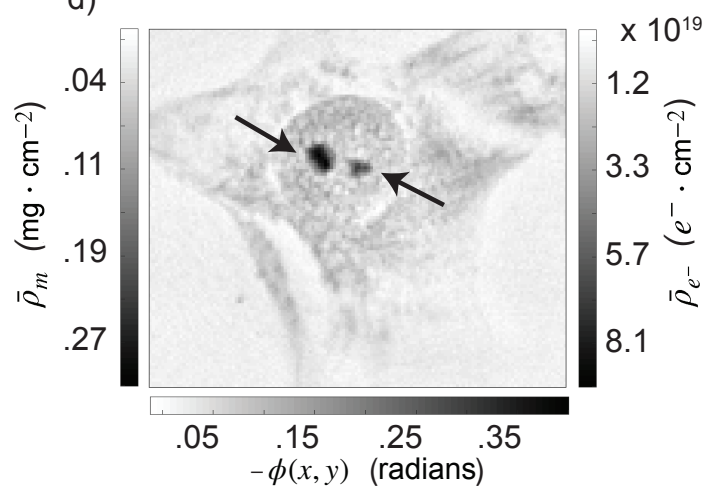

FiguRE 5.2: (a) X-ray dark field image of a typical NIH-3T3 fibroblast; here, the full data range of $\left[q_{0}, q_{\max }\right]=[0.038,0.867] \mathrm{nm}^{-1}$ is taken into account for calculating the dark field image. The color bar represents the total number of scattered photons per second. (b) Map of the exponent $\alpha$ for partial fitting ranges; $\left[q_{0}, q_{\min }\right]=[0.038,0.132] \mathrm{nm}^{-1},[0.038,0.085] \mathrm{nm}^{-1}$ and $[0.038,0.052] \mathrm{nm}^{-1}$ for the heterochromatin, euchromatin and cytoplasm ROIs, respectively. See Figure 5.4a for the real-space ROI definitions. (c) Map of the Porod constant $K$ for a partial data range; $\left[q_{\min }, q_{\max }\right]=[0.132,0.867] \mathrm{nm}^{-1},[0.085,0.867] \mathrm{nm}^{-1}$ and $[0.052,0.867] \mathrm{nm}^{-1}$ for the heterochromatin, euchromatin and cytoplasm, respectively. For both (b) and (c), the values are derived by fitting radial intensity profiles corresponding to individual scattering patterns. (d) The reconstructed phase map, rendered by reconstructing holograms recorded at a single defocused position using the RAAR [105] algorithm. The $2 \mathrm{D}$ projected mass and electron densities are also shown (grayscale bars on the left and right hand side, respectively). The scale bar in a) is $10 \mu \mathrm{m}$ and applies to all subfigures. 
Within the nucleus of a eukaryotic cell, $2 \mathrm{~nm}$ thick DNA double helices [14] are wrapped around octamers of histones, forming $10 \mathrm{~nm}$ diameter nucleosomes [28]. Nucleosomes spaced along the genome form a nucleofilament, which is often referred to as "beads on a string" [28]. Within the traditional "hierarchical helical folding model" [6], a nucleofilament is continuously packed into coils in a hierarchical manner. The first hierarchy forms a $30 \mathrm{~nm}$ diameter chromatin fiber, and subsequent hierarchy levels form coils up to $700 \mathrm{~nm}$ in size, which eventually lead to the formation of individual chromosomes approximately $1 \mu \mathrm{m}$ in size. The exact structure of the hierarchy levels is a topic of debate $[8,9]$.

Compared to the dark field image shown in Figure 5.2a, all subfigures of Figure 5.3 are noticeably different. One of the most pronounced features is the globular structure appearing in red and indicated by the white arrow in Figure 5.3a. This structure is prominently observed only for Fourier components corresponding to length scales in the range of 7 to $61 \mathrm{~nm}$. However, when regarding the adjusted dark field images shown in Figure 5.7a,b it becomes apparent that the scattered intensity actually increases as structures within the 35 to $61 \mathrm{~nm}$ range are imaged. From comparison to typical visible light phase contrast or differential interference contrast micrographs [134, 135], we interpret this region as a nucleolus. The diameter of a nucleolus is roughly $2 \mu \mathrm{m}$ [134], which corresponds well to the size of the intense regions in Figure 5.3a, b. A nucleolus is responsible for rRNA synthesis [136] and is composed of rDNA [137-139], RNA and several hundred types of proteins [134], including the phosphoprotein nucleolin [140]. This protein accounts for approximately $10 \%$ of the protein content within the nucleolus and has a diameter of roughly $15 \mathrm{~nm}[140,141]$, corresponding to the length scales detected here.

As shown throughout Figure 5.3b, f, as the probed length scales increase, the nucleolus structure becomes less pronounced while structures near the periphery of the nucleus become more prominent, as indicated by the white arrow in Figure 5.3c. The structures are primarily observed for Fourier components corresponding to length scales from 61 to $157 \mathrm{~nm}$. When viewing the adjusted dark field images (Figure 5.7b, f), the structures are observed throughout the 35 to $157 \mathrm{~nm}$ range. Regions of densely packed DNA that are found at the periphery of the nucleus are referred to as heterochromatin in literature $[135,142]$. In agreement with what we observe in the dark field images of Figure 5.3c, f and Figure 5.7c-f, higher-order chromatin structures have been observed on length scales ranging from 80 to $160 \mathrm{~nm}$ in fixed Drosophila melanogaster embryonic chromosomes [143]. Heterochromatin is necessary for both the expression of heterochromatic genes and the inhibition of the expression of euchromatic genes [144]. 
Throughout all length scales shown in Figure 5.3, we observe structures within the nucleus in addition to the nucleolus or heterochromatin. See, for example, structures which scatter approximately $2.5 \times 10^{7}$ photons $\cdot \mathrm{s}^{-1}$ in Figure $5.3 \mathrm{~b}$ and are represented by a light blue color on the corresponding color scale. Similar structures are observed for every subfigure of Figure 5.3 and all have a lower scattering power compared to the heterochromatin or nucleolus, as indicated by the relatively decreased number of detected photons. The regions likely contain loosely packed DNA, referred to as euchromatin. Euchromatin is known to be gene-rich and involved in active processes such as transcription [145]. We observe that at length scales of 139 to $157 \mathrm{~nm}$ (Figure 5.3f), euchromatin structures partially disappear. At these length scales, the transition from a smaller to larger hierarchy level may be occurring, thus the comparatively small structure sizes of loosely-packed euchromatin are not as clearly visible compared to the more-condensed heterochromatin. When observing the adjusted dark field images in Figure 5.7, it becomes clear that the scattered intensity from euchromatin is approximately the same for length scales within the 7 to $61 \mathrm{~nm}$ range, and on length scales from 61 to $139 \mathrm{~nm}$ the intensity increases. On length scales of 139 to $157 \mathrm{~nm}$, Figure $5.7 \mathrm{f}$ shows a decrease in intensity, similar to what is observed in Figure 5.3f.

a)

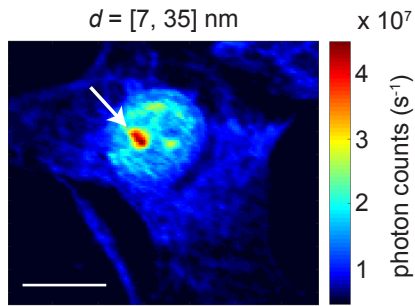

d)

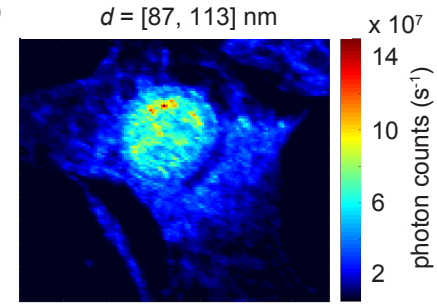

b)

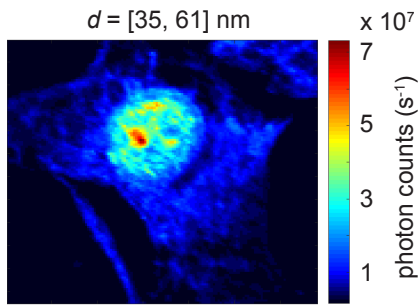

e)

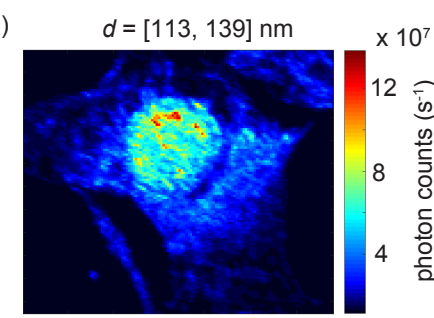

c)
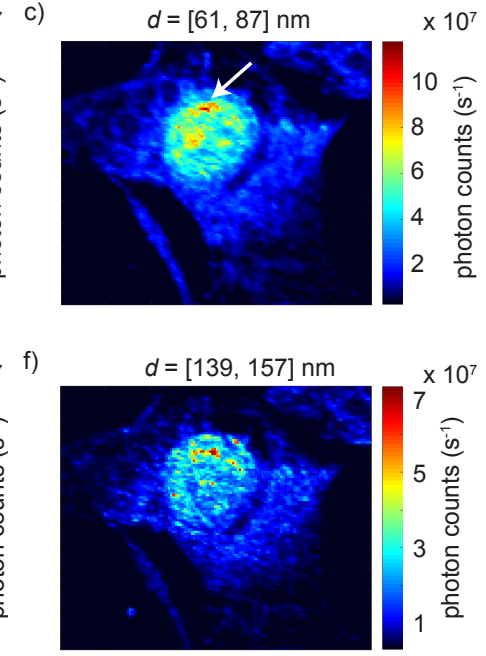

FIGURE 5.3: Calculated dark field images showing the granularity of the cell on different length scales. The color scale of each image ranges from the minimum to maximum number of detected photon counts for the corresponding $q$-range. See Figure 5.7 for a version of this plot with the same color scale used for all subfigures. The nucleolus structure is represented by the globular region of high scattering power, as indicated by the increase in detected photon counts, on length scales of (a) 7 to $35 \mathrm{~nm}$ (white arrow) and (b) 35 to $61 \mathrm{~nm}$. Heterochromatin is represented by regions of high scattering power at the periphery of the nucleus, and is observed on length scales of (c) 61 to $87 \mathrm{~nm}$ (white arrow), (d) 87 to $113 \mathrm{~nm}$, (e) 113 to $139 \mathrm{~nm}$ and (f) 139 to $157 \mathrm{~nm}$. Euchromatin fills the remainder of the nucleus on all length scales and is depicted in a light blue color. The scale bar is $10 \mu \mathrm{m}$ and applies to all subfigures. 
From the various dark field images shown in Figure 5.3, structures are observed throughout all length scales, namely the nucleolus, heterochromatin and euchromatin. These structures are composed of material scattering with Fourier components corresponding to a size range from 7 to $157 \mathrm{~nm}$ and represent a portion of the length scales covered throughout the entire DNA packaging process, i.e., $2 \mathrm{~nm}$ to $1 \mu \mathrm{m}$. Subsequent analysis is based on defining ROIs by visual inspection of a dark field image. The dark field image shown in Figure 5.2a is calculated using the largest $q$-range possible, corresponding to all structures within the $[7,157] \mathrm{nm}$ range, and represents the sum of all dark field images shown in Figure 5.3. For this reason, Figure 5.2a is used to define the ROIs. The ROIs, as shown in Figure 5.4a, represent euchromatin in blue and the cytoplasm in gray. A background ROI (black) is selected and used for subsequent data correction. We combine the nucleolus and heterochromatin structures into one ROI (orange, in the following referred to as heterochromatin), as a separate analysis of the two regions results in only minor differences in the values of $\alpha$ and $K$, see Figure 5.8.

To characterize each ROI, the corresponding 2D scattering patterns are averaged, subsequently azimuthally integrated and the resulting intensity values $I$ are plotted with respect to the magnitude of the scattering wave vector $q$. We exploit plots of $I(q)$ to probe structural information concerning the morphology and aggregation state of biological material inside the cells. To this end, $I(q)$ data are background corrected and fitted using a power law [69, 84]

$$
I(q)=K q^{\alpha}+B
$$

where the constant $B$ accounts for inelastic and incoherent scattering. Note that the units of eq. 5.3 are only well-defined when the exponent is an integer. Figure 5.4b shows an example of fitted $I(q)$ curves; these curves exhibit one power-law regime. Interestingly, 24 of the 33 cells analyzed have $I(q)$ curves which exhibit two power-law regimes with an apparent "kink" around $q \sim 0.1 \mathrm{~nm}^{-1}$ where the slopes transition from higher towards lower values. Figure 5.9c shows an example of such $I(q)$ curves. Additionally, Figure 5.6c, d shows phase contrast micrographs of the same cell in the chemically fixed and lyophilized state, respectively. This "kink" phenomenon has also been observed previously [92] for a different cell type. 
a)

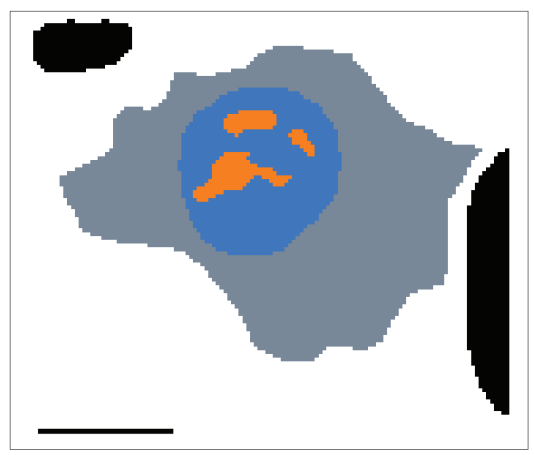

c)
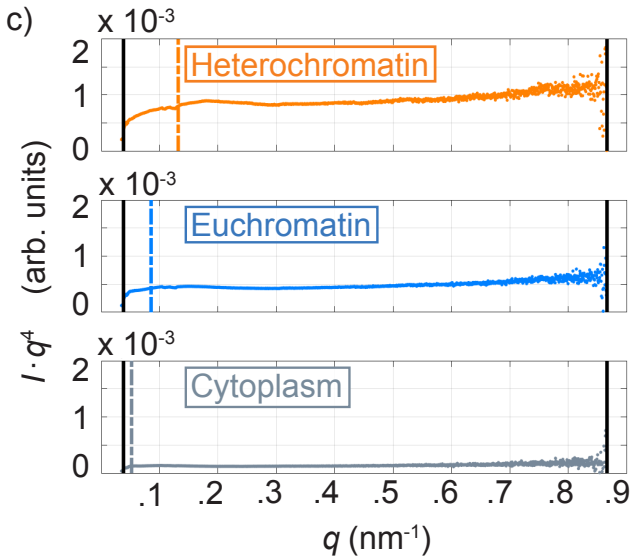

b)

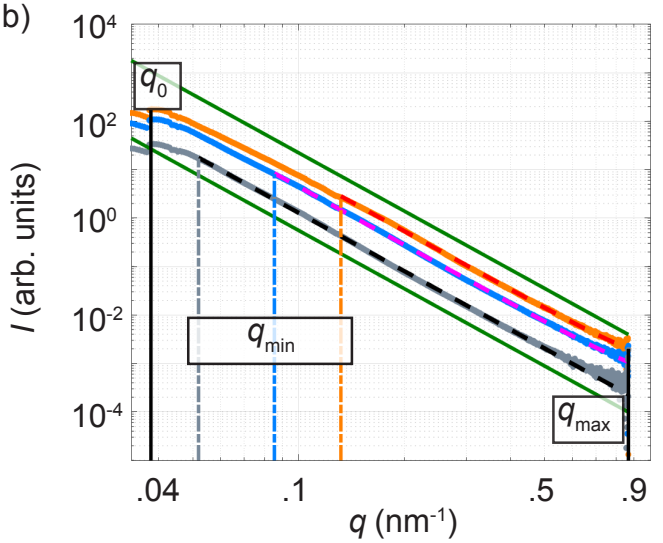

d)

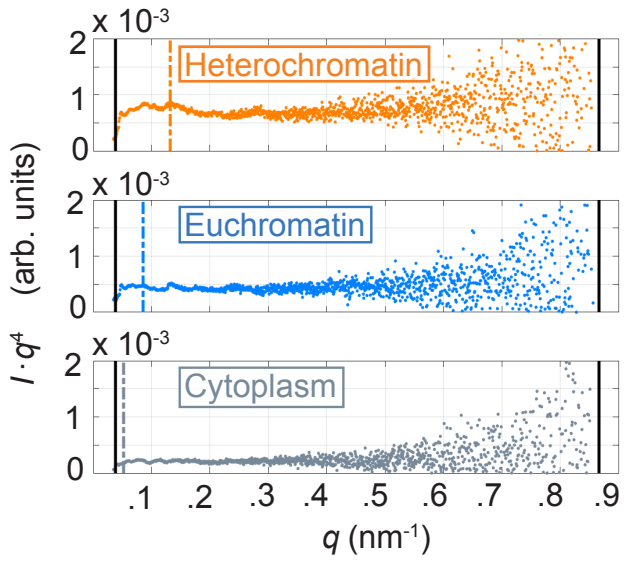

Figure 5.4: (a) Manually selected regions of interest. The heterochromatin and euchromatin regions are shown in orange and blue, respectively, the cytoplasm in gray and the background in black. The scale bar is $10 \mu \mathrm{m}$. (b) The scattering patterns belonging to each region are averaged, azimuthally integrated, background-corrected and plotted against the absolute value of the scattering vector $q$. The radial intensity profiles are fitted using a power law decay (eq. 5.3) from $q_{\min }$ to $q_{\max }$, as shown by the red and black dashed lines. The vertical dashed lines represent the $q_{\min }$ value of the respective ROI. The solid black lines at $q=0.038 \mathrm{~nm}^{-1}$ and $q=0.866 \mathrm{~nm}^{-1}$ represent $q_{0}$ and $q_{\max }$, respectively. The solid green lines are proportional to $q^{-4}$ and serve only as a visual aid to the overall $I(q)$ decay. (c) $1 \mathrm{D}$ radial intensity profiles, corresponding to the averaged scattering patterns of the regions of interest, plotted as $I \cdot q^{4}$ vs. $q$ (Porod plot). The left and right vertical black lines represent $q_{0}$ and $q_{\max }$, respectively. The vertical dashed lines represent $q_{\min }$. Samples that can be described using Porod's law exhibit a characteristic plateau in the Porod plot. (d) Porod plots corresponding to a single, typical scattering pattern from each region of interest. $I \cdot q^{4}$ values below zero are omitted.

The exponent $\alpha$ describes the dimensionality, shape and surface roughness of the sample. For smooth, three-dimensional objects, we expect $\alpha=-4$, for two-dimensional objects $\alpha=-2$ and for one-dimensional objects $\alpha=-1$ [70]. Non-integer values of $\alpha$ may be indicative of a fractal nature of the scatterers, e.g., a rough surface. To access local structural information, resolved in real space on the length scale of the beamsize, $I(q)$ curves belonging to individual scattering patterns are analyzed. A map of $\alpha$ values for each scan position, determined by using eq. 5.3 to fit individual $I(q)$ curves from $q_{0}$ to 
$q_{\min }$, where $q_{0}$ is fixed and $q_{\min }$ is unique for each ROI, is shown in Figure 5.2b. For the single cell shown in Figure $5.2 \mathrm{~b}$ the heterochromatin ROI is fitted from $\left[q_{0}, q_{\min }\right]=$ $[0.038,0.132] \mathrm{nm}^{-1}$, relevant to structures with length scales of $[48,157] \mathrm{nm}$ in real space, and a median value of $\alpha=-3.5$ is found. Similarly, the euchromatin ROI is fitted from $[0.038,0.085] \mathrm{nm}^{-1}$, relevant to length scales of $[70,157] \mathrm{nm}$ in real space, and a median value of $\alpha=-3.4$ is found. The median values indicate that the material distributed within the heterochromatin and euchromatin ROIs have similar local morphology. In the map shown in Figure 5.2b one globular region resembling a nucleolus is faintly observed, as indicated by the white arrow. Compared to the surrounding DNA, the nucleolus shows increased $\alpha$ values, indicating that it has some degree of morphological difference compared to neighboring structures. This difference could be associated with the various proteins known to compose the nucleolus. Within the cytoplasm of the cell shown in Figure 5.2b we find very noisy values, which is most likely explained by the small $\left[q_{0}, q_{\text {min }}\right]=[0.038,0.052] \mathrm{nm}^{-1}$ range fitted for this particular cell. For the $N=$ 33 cells, the average $\left[q_{0}, q_{\text {min }}\right]$ range fitted for the cytoplasm ROIs is $[0.038,0.084] \mathrm{nm}^{-1}$.

Even though eq. 5.3 may be used to characterize data in the case of $\alpha \neq-4$, the equation is only referred to as Porod law $[70,84]$ in the special case of $\alpha=-4$. As a consequence, $\alpha$ and $K$ are termed the Porod exponent and Porod constant, respectively. To determine the $q$-range in which $\alpha=-4$, we systematically, and for each ROI of every cell, separately fit the averaged $I(q)$ curve with different $q$-ranges using eq. 5.3 and compare the fit coefficients for $\alpha$ to the theoretical value of -4 . The largest $q$ range which obeys the enforced threshold condition (see Materials and Methods) defines $\left[q_{\min }, q_{\max }\right]$. Alternatively, instead of plotting the intensity $I$ versus the scattering vector $q$ to determine $\left[q_{\min }, q_{\max }\right]$, it is possible to use so-called Porod plots [146] of $I \cdot q^{4}$ vs. $q$. In this representation, a characteristic plateau appears in the range $\left[q_{\min }, q_{\max }\right]$. Porod plots of data averaged over each ROI are shown in Figure 5.4c, and typical individual data sets from each ROI are shown in Figure 5.4d.

When comparing our data to the literature, we obtain a consistent picture. For lyophilized samples, values of $\alpha \simeq-4$ are typically reported [31, 91, 93, 108]. However, previous work [92] comparing chemically fixed-hydrated cells and living cells has shown that the sample preparation procedure has a considerable influence on measured values of $\alpha$. Systematically higher values of $\alpha$, i.e. closer to zero, for both sample types are reported and values in the range of $-4.0<\alpha<-3.0$ and $-3.0<\alpha<-2.5$, for chemically fixed and living cells, respectively, were found.

If $\alpha$ is fixed at -4 , the Porod constant $K$ depends on the electron density contrast $\Delta \rho_{\mathrm{e}^{-}}$ of the sample and the surface area $S$ of the interface between scatterers and environment, 
and thus the aggregation state of the scatterers [69, 70]:

$$
K=2 \pi\left(\Delta \rho_{\mathrm{e}^{-}}\right)^{2} S
$$

Equation 5.4 assumes both the sample and background are composed of homogeneous electron densities where $\rho_{\mathrm{e}^{-}}$, sample $\neq \rho_{\mathrm{e}^{-}}$, bg Thus, it is not directly applicable to biological samples but serves as an aid to understand the relationship between $\Delta \rho_{\mathrm{e}^{-}}$and $S$. A large Porod constant value can be due to (i) a large interface area $S$, (ii) a large electron density contrast $\Delta \rho_{\mathrm{e}^{-}}$between the two phases of the sample or (iii) a combination of both. A map of the Porod constant $K$, derived by using eq. 5.3 to fit individual $I(q)$ profiles within $\left[q_{\min }, q_{\max }\right]$, where $\alpha=-4$, is shown in Figure 5.2c. The DNA distribution at the periphery of the nucleus, as indicated by the red arrow in Figure 5.2c, is mostly in agreement with the heterochromatin distribution observed throughout the dark field images shown in Figure 5.3b, f and Figure 5.7b, f. As heterochromatin is tightly packed chromatin, resulting in a larger electron density compared to euchromatin, it is reasonable to expect the heterochromatin distributions in the Porod constant map and dark field images to resemble each other. In agreement with the dark field images shown in Figure 5.3a, b and Figure 5.7a, b, a globular region resembling a nucleolus is distinguishable in the $K$ map, as indicated by the white arrow in Figure 5.2c. Since $K$ is only linearly proportional to $S$ but proportional to the square of $\Delta \rho_{e^{-}}$, and a nucleolus is densely filled with proteins, we expect to observe the nucleolus in the $K$ map. However, it is additionally possible that the total interface area $S$ of the material within the nucleolus is large.

\subsubsection{Quantitative Density Measurements in the Cell Nucleus}

From the discussion above it becomes clear that scanning SAXS measurements provide information about the morphology and aggregation state of nuclear material, however, the electron density $\Delta \rho_{e^{-}}$is not quantitatively accessible. Thus, we combine the scanning SAXS measurements with X-ray in-line holography to access both the projected electron density and projected mass density. By contrast to scanning SAXS, which is sensitive to structures of typical length scales, holography is a full-field imaging technique sensitive to the integrated electron density of material along the propagation direction of the X-rays.

In the holography setup, shown schematically in Figure 5.1b, the KB-focused X-rays are coupled into a waveguide [106] that acts as a quasi point source $(\leq 20 \mathrm{~nm})$, emitting a highly divergent wavefront which is ideally suited for near-field imaging. Compared to the KB-focused beam the waveguide increases the numerical aperture of the system, 
thus increasing the imaging resolution. The sample is placed at a series of defocused positions, $X_{1}$, and full-field holograms are recorded at a sample-to-detector distance of $X_{2}$. Holograms are recorded at multiple distances to account for the zero-crossings of the phase contrast transfer function produced when imaging weakly-absorbing objects with a slowly-varying phase, e.g., biological samples [99]. By adjusting the geometric magnification of the system, given by $M=\frac{X_{1}+X_{2}}{X_{1}}$, the FOV can be tailored to accommodate the imaging of either single or a group of multiple cells. Thereby, the effective pixel size of the holograms, given by $p_{\text {eff }}=\frac{p}{M}$, where $p$ is the pixel size of the detector, is varied.

We initiate the phase map by numerically processing the acquired holograms via the CTF algorithm. The resulting reconstructed phase map is then employed to define the support constraint used in the RAAR algorithm. In short, one iteration of the reconstruction process begins by propagating the measured intensity from the detector plane to the object (sample) plane. An object support is subsequently applied, which accounts for the shape of a spatially resolvable object. Here, the phase map rendered from the CTF reconstruction is used to define the object support and is chosen to account for both the cell body as well as its surrounding background. The waveform is then propagated towards the detector plane, where its amplitude is replaced by the square-root of the measured intensity, i.e., the modulus constraint, and is subsequently propagated back to the object plane. The iteration round is now complete. This waveform then serves as the starting point for the next iteration. The object support is held constant throughout the entire iterative process.

To correlate the holographic and scanning SAXS data in a pixel-wise manner, all resulting maps from the holography and scanning SAXS measurements must have the same pixel size and FOV. To this end, we register the reconstructed phase maps to the dark field images, thus rendering them suitable for direct comparison. An example of a registered 2D reconstructed phase map, denoted as $\phi(x, y)$, is shown in Figure 5.2d. The nucleus and cell body are clearly distinguishable. In particular, two globular regions are observed, as indicated by the black arrows. This phase map is reconstructed using holograms recorded at a single distance; the recording of holograms at multiple distances is performed to ensure the optimal phase retrieval for the CTF algorithm, which subsequently serves as the support constraint for the single distance RAAR algorithm.

To determine the phase contribution of the cell itself, the median value of the background region, shown in black in Figure 5.4a, is determined and subtracted from the rest of the image, i.e., $\phi(x, y)=\phi_{\text {sample }}-\phi_{\mathrm{bg}}$; the resulting values of $\phi(x, y)$ are used for subsequent analysis. The median value of the background region for the cell shown in Figure 5.2d is -0.014 radians. The reconstructed phase is directly related to the $2 \mathrm{D}$ projected electron 
density (electron density per area) [101], $\bar{\rho}_{e^{-}}(x, y)$, via:

$$
\bar{\rho}_{e^{-}}(x, y) \simeq \frac{-\phi(x, y)}{r_{e} \lambda_{0}}
$$

where $r_{e}$ and $\lambda_{0}$ denote the classical electron radius and the X-ray incident wavelength, respectively. The projected electron density is related to the $2 \mathrm{D}$ projected mass density $[33,131], \bar{\rho}_{m}(x, y)$, via:

$$
\bar{\rho}_{m}(x, y) \simeq\left(\frac{-2 u}{r_{e} \lambda_{0}}\right) \cdot \phi(x, y)
$$

where $u$ is the atomic mass unit. The factor of 2 is dependent on the chemical composition of the sample. For cellular constituents with an average empirical formula of $\mathrm{H}_{50} \mathrm{C}_{30} \mathrm{~N}_{9} \mathrm{O}_{10} \mathrm{~S}_{1}$ the factor of 2 valid [33]. As $\phi, \bar{\rho}_{e^{-}}$and $\bar{\rho}_{m}$ are proportional to each other, Figure 5.2d, in addition to the measured phase shift, also shows the $2 \mathrm{D}$ projected electron and projected mass density maps, see scale bars on the right and left hand side, respectively. Overall, we find median values of $\bar{\rho}_{e^{-}}$for the heterochromatin, including the nucleoli, and euchromatin regions to be $2.1 \times 10^{19}$ and $1.1 \times 10^{19} \mathrm{e}^{-} \cdot \mathrm{cm}^{-2}$ with corresponding $\bar{\rho}_{m}$ values of 0.07 and $0.04 \mathrm{mg} \cdot \mathrm{cm}^{-2}$, respectively. Within the cytoplasm, we find median values of $\bar{\rho}_{e^{-}}=5.1 \times 10^{18} \mathrm{e}^{-} \cdot \mathrm{cm}^{-2}$ and $\bar{\rho}_{m}=0.02 \mathrm{mg} \cdot \mathrm{cm}^{-2}$. The reported values of the projected mass density are comparable to lyophilized Deinococcus radiodurans [33] and lyophilized bacterial endospores [131].

In our holography setup the accessed momentum transfer $q$ is shifted towards smaller values, i.e., larger real space structures, compared to the scanning SAXS experimental setup, which has a similar sample-to-detector distance. To estimate the resolution of the reconstructed phase map shown in Figure 5.2d, the power spectral density (PSD) is calculated and azimuthally averaged. The resolution is approximated by the transition from signal to noise and, for our experimental setup, is found around $q=0.119 \mathrm{~nm}^{-1}$, corresponding to a real space resolution of $53 \mathrm{~nm}$. We can thus conclude that the DNA structures observed in the projected electron density map correspond to length scales of at least $53 \mathrm{~nm}$. Figure 5.10 shows a comparison between the PSD and a typical $I(q)$ profile derived from the scanning SAXS measurements. We observe the overall slope of the PSD to be $\sim-3$, in contrast to the $I(q)$ slope of $\sim-4$. As also shown in Figure 5.10, the combination of holography and scanning SAXS enables access to $q$-values spanning three orders of magnitude [108]. However, as a direct consequence of the different $q$ ranges accessed by each imaging modality with only little overlap, we cannot directly combine the data and quantify of the interface area $S$ via eq. 5.4 by inserting $\bar{\rho}_{e^{-}}(x, y)$. Holography is sensitive to the collective electron density of the probed material, thus indicating that the two globular regions in the projected electron density map shown in Figure 5.2d are dense regions. In agreement with visible light phase contrast images [134, 
135] and our interpretation from the dark field images shown in Figure 5.3a, b and Figure $5.7 \mathrm{a}, \mathrm{b}$, we suggest that these regions are nucleoli. In contrast to the projected electron density map, where two nucleoli are observed, only one nucleolus is prominently observed in the Porod constant $K$ map (Figure 5.2c). Interestingly, only the left nucleolus is pronounced in both maps; the right nucleolus is less pronounced in the $K$ map. The most likely explanation for the variation between the two maps is that the local material in the right nucleolus is composed of dense material that is aggregated into a volume with a small interface area $S$. According to eq. 5.4, a small interface area would decrease $K$.

\subsubsection{DNA Aggregation and Density for a Cell Ensemble}

So far, we have discussed typical results for a particular cell that we investigated in this study. The same analysis was performed for an ensemble of $N=33$ cells. Figure 5.5 shows distributions of all variables discussed above for all measured positions within all cells, separately for the cytoplasm and the two nuclear regions, thus adding statistical relevance to our results. A total of 10425 scattering patterns were analyzed for the heterochromatin region, 36655 for the euchromatin region and 106460 for the cytoplasm. The median values of the distributions are listed in Table 5.2. As shown in Figure 5.5a, for data that do not obey Porod's law, i.e., data within $\left[q_{0}, q_{\min }\right]$ where $\alpha \neq-4$, we find similar median values for $\alpha$ throughout the three ROIs, albeit slightly smaller for the cytoplasm regions. Two-component Kolmogorov-Smirnov [147] (KS) tests were performed with a null hypothesis that the three distributions shown in Figure 5.5a do not significantly differ. At the $5 \%$ significance level the null hypothesis is rejected, indicating all three distributions are significantly different from one another. 

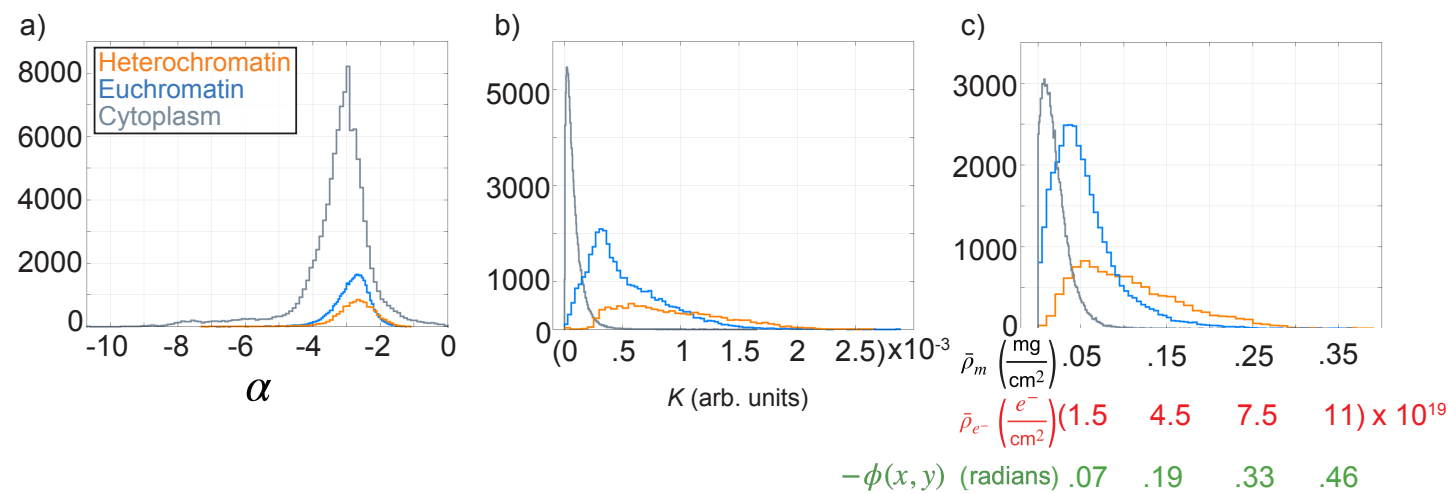

FiguRe 5.5: Histograms of variables from fitting individual $I(q)$ profiles of $N=33$ cells. The $y$-axes represent the number of counts. The heterochromatin and euchromatin distributions are shown in orange and blue, respectively. The cytoplasm distributions are gray. (a) The exponent $\alpha$, eq. 5.3, from fitting $I(q)$ profiles in the $\left[q_{0}, q_{\text {min }}\right]$ range where $\alpha \neq-4$. (b) The Porod constant $K$, derived from fitting profiles in the $\left[q_{\min }, q_{\max }\right]$ range where $\alpha=-4$. (c) The projected mass density (black axis labels), projected electron density (red axis labels) and phase shift (green axis labels). The number of bins is calculated using Scott's rule [148]. All median values are listed in Table 5.2.

The distributions of the Porod constant $K$, derived by fitting data within $\left[q_{\min }, q_{\max }\right]$ where $\alpha=-4$, are shown in Figure 5.5b. We find that for each ROI the distribution of $K$ values varies, similar to the map shown in Figure 5.2c. The largest median value of $K$ is reported for the heterochromatin regions of the cells (orange), indicating that the product of the interface area $S$ and projected electron density contrast squared is a factor of 3.4 larger than for the euchromatin regions (blue) and 16.4 larger than for the cytoplasm regions (gray).

As the reconstructed phase $\phi$, projected electron density $\bar{\rho}_{e^{-}}$and projected mass density $\bar{\rho}_{m}$ are all directly related to one another, differing only by constant factors (see eqs. 5.5 and 5.6), Figure 5.5c shows the distributions of all three variables. We find that within the heterochromatin ROIs of all 33 cells, there is a factor of 2.1 more material than within the euchromatin regions, as described by the relative increase in both the projected electron density and projected mass density. Our calculated factor of 2.1 is on the same order of magnitude as in the confocal microscopy study presented by [149], who concluded that heterochromatin of living HeLa cells is a factor of 1.4 as condensed as euchromatin. Compared to the cytoplasm regions, we find the heterochromatin regions to contain a factor of 7.1 more material. 
TABLE 5.1: Summary of the parameters probed by combined scanning SAXS and Xray holography, including the nuclear structures accessible by each of the analyses and the physical property probed by the respective method.

\begin{tabular}{|ccc|}
\hline Analysis approach & Nuclear structures accessed & Physical property probed \\
\hline \hline Dark field & $\begin{array}{c}\text { Nucleoli, } \\
\text { Heterochromatin }, \\
\text { Euchromatin }\end{array}$ & $\begin{array}{c}\text { Total scattering, } \\
\text { Fourier components relevant } \\
\text { for specific length scales }\end{array}$ \\
\hline Power law exponent $\alpha$ & (Nucleoli) & Morphology \\
\hline Porod constant K & $\begin{array}{c}\text { Nucleoli, } \\
\text { Heterochromatin, } \\
\text { (Euchromatin) }\end{array}$ & $\begin{array}{c}\text { Aggregation state, } \\
\text { Interface area }\end{array}$ \\
\hline Phase shift & Nucleoli & $\begin{array}{c}\text { Electron/ } \\
\text { mass density }\end{array}$ \\
\hline
\end{tabular}

\subsection{Summary and Conclusions}

To summarize, we exploit the short wavelength and high penetration depth of X-rays to image whole, intact cells. In particular, we combine X-ray holography and scanning SAXS, including the analysis of dark field representations and the power law fits of intensity profiles, $I(q)$. By this three-fold view on a complex biological system, the cell nucleus, we obtain access to the projected electron and mass density, length scales of the scatterers, and aggregation and morphology of nuclear material. Separate dark field representations for different ranges of momentum transfer reveal nuclear regions containing nucleoli, heterochromatin or euchromatin, respectively, as the method is highly sensitive to the prominent length scale of the scatterers. Analysis of the power law exponent $\alpha$, which gives rise to differences in scatterer morphology, cannot clearly distinguish the different nuclear regions, which is typical for complex biological matter. By contrast, the Porod constant $K$, a measure for the aggregation of the scatterers, reveals regions dominated by heterochromatin and the locations of some, but not all, nucleoli. Finally, $\mathrm{X}$-ray holography is able to clearly distinguish the nucleoli by quantitative electron and mass density analysis, see summary in Table 5.1.

Turning this line of arguments around, for the methods we employed here, comparatively loosely packed euchromatin is visible only in the scaled dark field representations, denser, more aggregated heterochromatin in dark field and $K$ maps, and finally, the very dense, strongly aggregated nucleoli in all three analyses. From a biological point of view, we find that nucleoli are the densest structures in the nucleus, scattering mostly on length scales up to about $60 \mathrm{~nm}$, indicating the existence of structures in this size range, possibly proteins. While the density of heterochromatin and euchromatin is similar and lower than for nucleoli, as revealed by X-ray holography, heterochromatin mostly scatters on length scales above $35 \mathrm{~nm}$ and euchromatin scatters on all probed length scales. The 
Porod constant reveals that compared to euchromatin, heterochromatin and nucleoli are more aggregated. In agreement with literature [135, 142, 143], we find heterochromatin to be located near the periphery of the nucleus and euchromatin to fill all other regions that are not occupied by heterochromatin or the nucleoli. Our study highlights the importance of combined imaging approaches that capture multiple length scales for the characterization of complex biological systems. The combination of scanning SAXS and $\mathrm{X}$-ray holography is straightforward and can be realized in a single experimental setup and can easily be applied to other biological systems.

\subsection{Supporting Information}

\subsubsection{Visible Light Phase Contrast Microscopy}

Figure 5.6 shows visible light phase contrast images of chemically-fixed and lyophilized cells adhered to $\mathrm{Si}_{3} \mathrm{~N}_{4}$ membranes. Images were acquired using an inverted microscope (IX81, Olympus, Hamburg, Germany) equipped with a Retiga 6000 Monochrome camera (QImaging, Tucson, AZ, USA; $2752 \times 2208$ pixels $(\mathrm{H} \times \mathrm{V})$, pixel size: $\left.4.54 \times 4.54 \mathrm{\mu m}^{2}\right)$ and a $20 \times$ objective (UCPlanFLN, NA=0.7; Olympus). 
a)

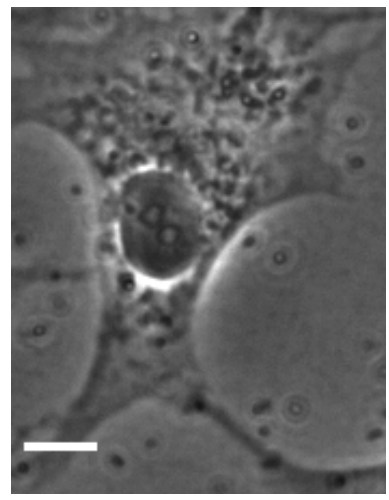

c)

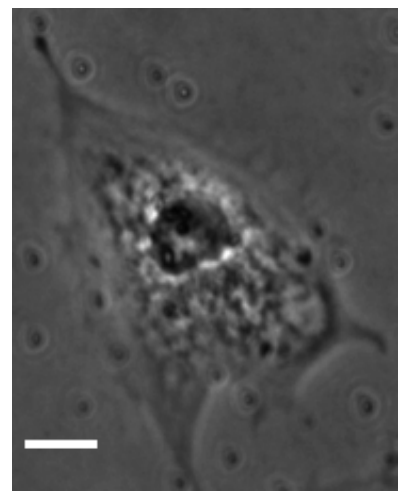

b)

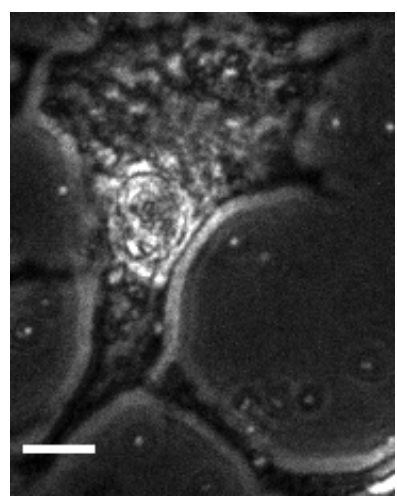

d)

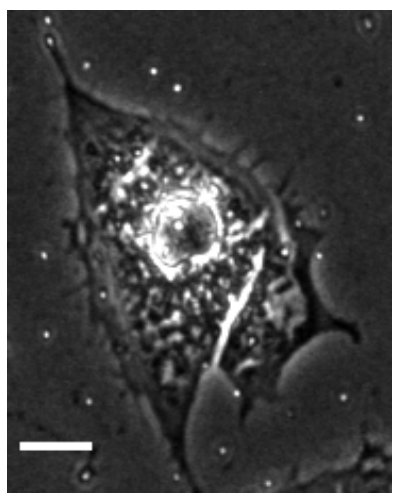

Figure 5.6: Visible light phase contrast images of NIH-3T3 fibroblasts adhered to $\mathrm{Si}_{3} \mathrm{~N}_{4}$ membranes. The cell shown in Figure 5.2a in the a) chemically-fixed and b) lyophilized state. The cell shown in Figure 5.9 in the c) chemically-fixed and d) lyophilized state. All scale bars are $10 \mu \mathrm{m}$.

\subsubsection{Comparison of Dark Fields}

Figure 5.7 shows the same dark field images shown in Figure 5.3 only with adjusted color scales. All subfigures in Figure 5.7 have the same color scale adjusted to account for the minimum and maximum number of detected photons throughout all subfigures. 
a)

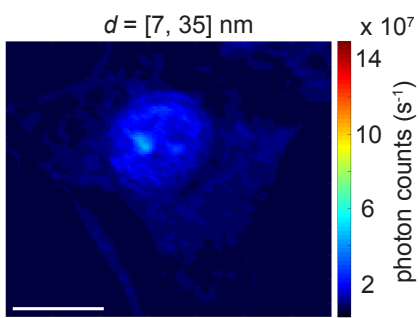

d)

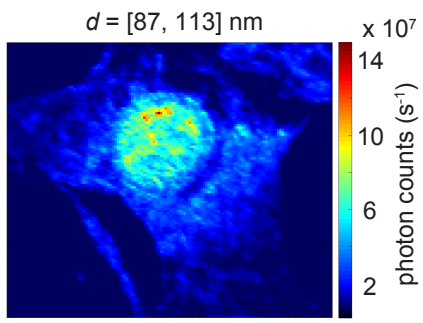

b)

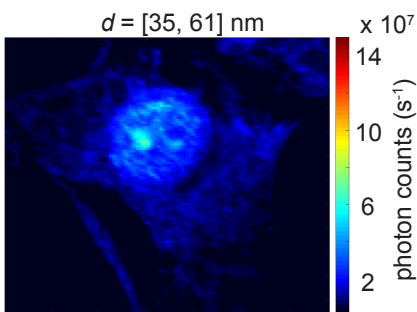

e)

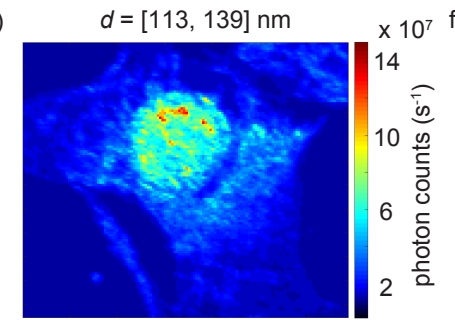

$d=[61,87] \mathrm{nm}$

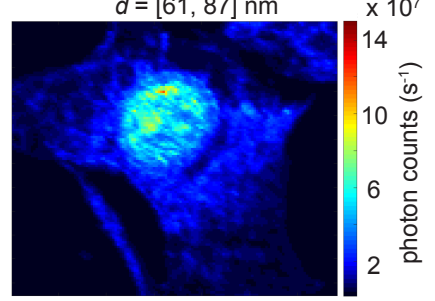

$d=[139,157] \mathrm{nm}$

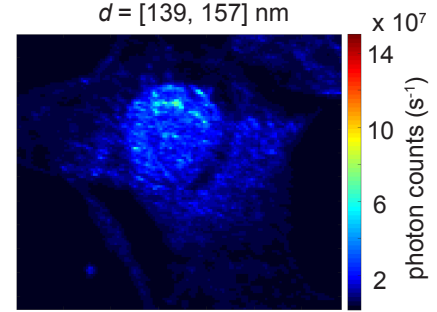

Figure 5.7: Dark field images of the cell shown in Figure 5.3. Here, all images have the same color scale. Compared to all other subfigures, the contrast of subfigure e) is lower due to high-intensity streaks of the primary X-ray beam localized within the corresponding $q$-range. The scale bar is $10 \mu \mathrm{m}$ and applies to all subfigures.

\subsubsection{Nucleoli Region of Interest}

As discussed in section 5.3.1, the "heterochromatin ROI" contains the heterochromatin and nucleoli structures. To justify this combination, a ROI of only the nucleoli is created and analyses of the heterochromatin ROI with and without the nucleoli ROI included are compared. The diffraction patterns belonging to each ROI are averaged and azimuthally integrated to obtain 1D $I(q)$ profiles. The exponent $\alpha$, derived by fitting $I(q)$ in the $\left[q_{0}, q_{\min }\right]$ range, is determined. For the heterochromatin ROI with and without the nucleoli included, $\left[q_{0}, q_{\min }\right]=[0.038,0.132] \mathrm{nm}^{-1}$ and $[0.038,0.079] \mathrm{nm}^{-1}$, respectively, and values of -3.5 and -3.6 are found. The Porod constant $K$ is determined by fitting in the range of $\left[q_{\min }, q_{\max }\right]$, where the Porod exponent $\alpha=-4$ is found. For the heterochromatin ROI with and without the nucleoli included, $\left[q_{\min }, q_{\max }\right]=[0.132,0.867]$ $\mathrm{nm}^{-1}$ and $[0.079,0.867] \mathrm{nm}^{-1}$, respectively, and values of $7.6 \times 10^{-4}$ and $7.1 \times 10^{-4}$ are found. Fits of the $I(q)$ profiles are shown in Figure 5.8c. 
a)

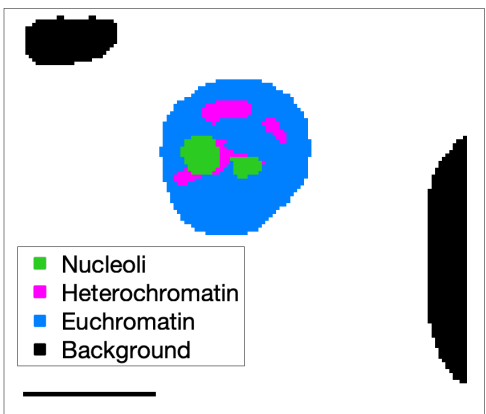

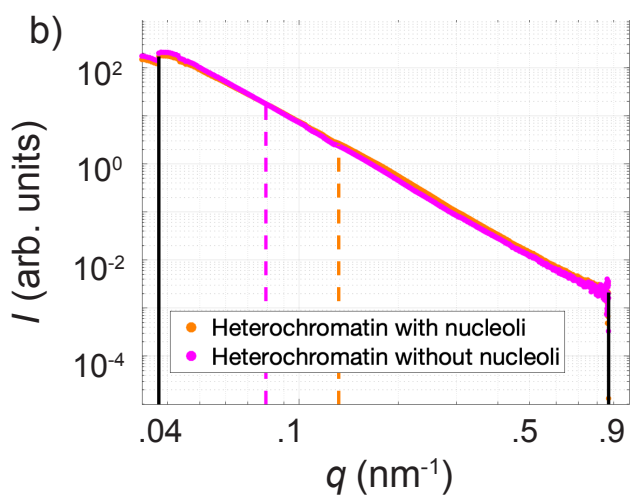

c)
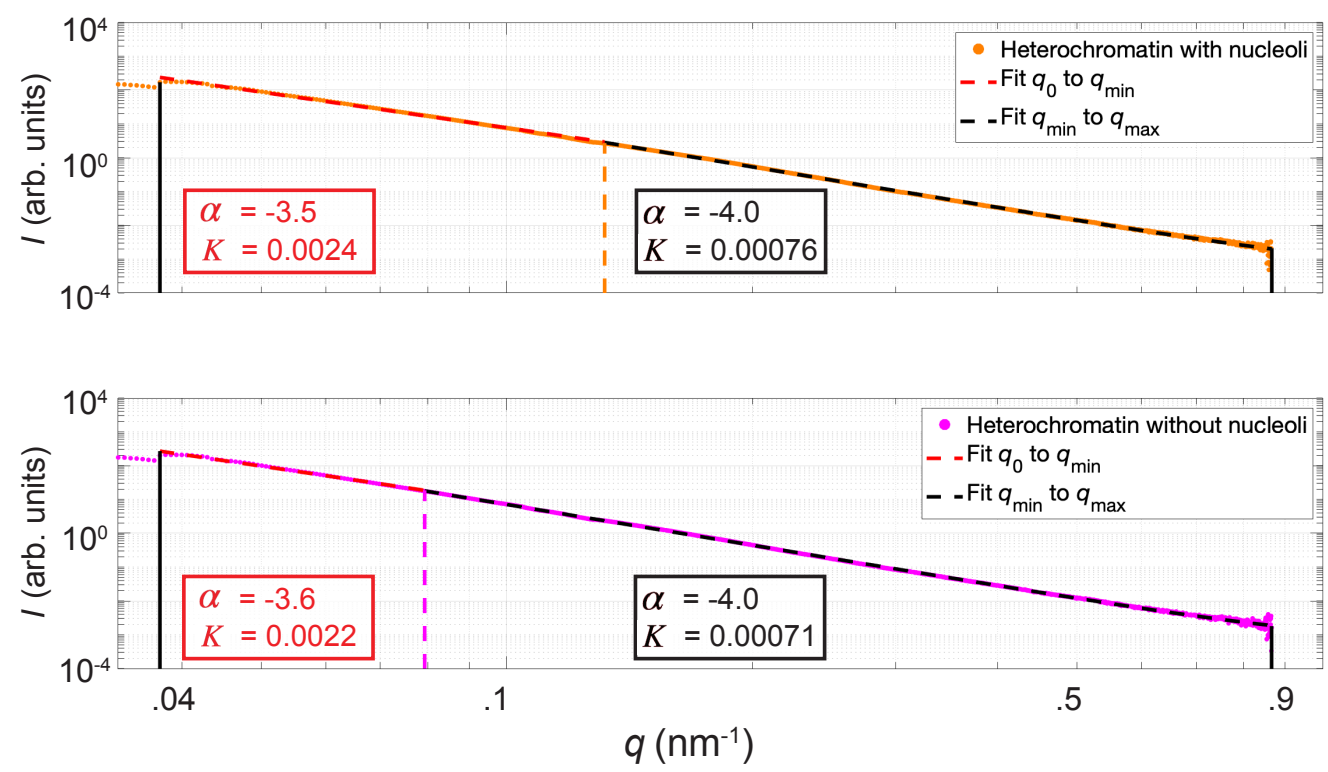

FIGURE 5.8: a) ROIs selected from the reconstructed phase map in Figure 5.4d. The heterochromatin ROI (purple) does not include any of the nuclei ROI (green). b) Corresponding background-corrected radial intensity $I(q)$ profiles. The purple line corresponds to the heterochromatin ROI shown in a). The orange line depicts the heterochromatin $I(q)$ profile shown in Figure 5.4b and corresponds to the combined heterochromatin and nucleoli ROIs. The vertical dashed lines represent $q_{\min }$ for the respective ROI. The solid, black vertical lines at $q=0.038 \mathrm{~nm}^{-1}$ and $q=0.867 \mathrm{~nm}^{-1}$ represent $q_{0}$ and $q_{\max }$, respectively. c) Fitted $I(q)$ profiles. The red and black dashed lines represent the fits of $I(q)=K q^{\alpha}+B$ for the $\left[q_{0}, q_{\min }\right]$ and $\left[q_{\min }, q_{\max }\right]$ ranges, respectively.

\subsubsection{Supplementary Cell}

Figure 5.9 is similar to both Figure 5.2 and Figure 5.4a,b and represents a different example cell. This cell exhibits a distinguishable "kink" around $q \simeq 0.1 \mathrm{~nm}^{-1}$ in its $I(q)$ profiles. The dark field image calculated using the entire usable data range $\left[q_{0}, q_{\max }\right]=[0.04,0.87] \mathrm{nm}^{-1}$, which corresponds to $[7,157] \mathrm{nm}$ in real space is shown. 
Additionally, the corresponding manually selected regions of interest, averaged and background corrected radial intensity $I(q)$ profiles, the exponent $\alpha$, the Porod constant $K$, and the reconstructed phase, projected mass and projected electron densities are presented. Similar figures bleoning to the 33 cells analyzed in this study are presented in Appendix B 
a)
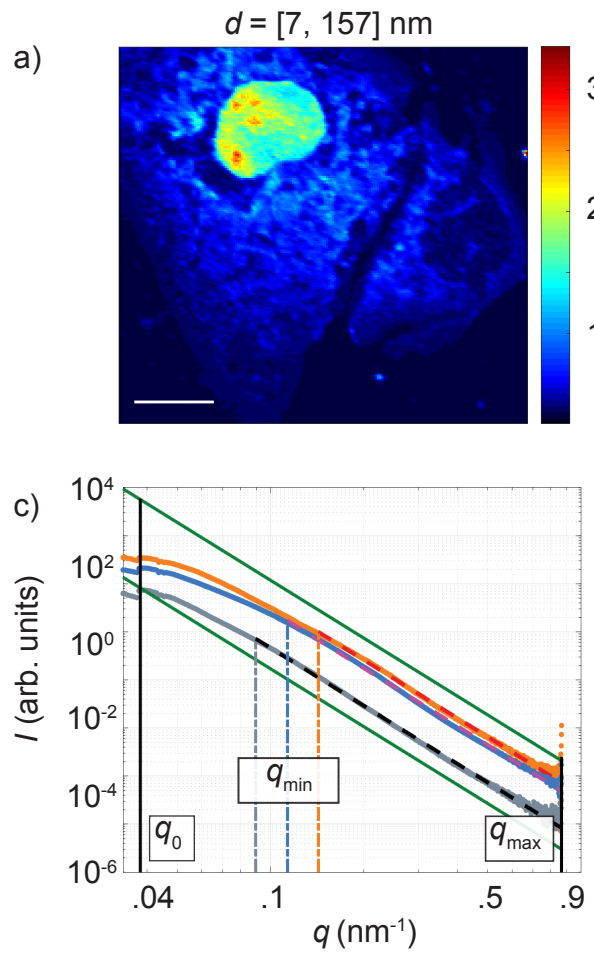

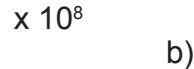

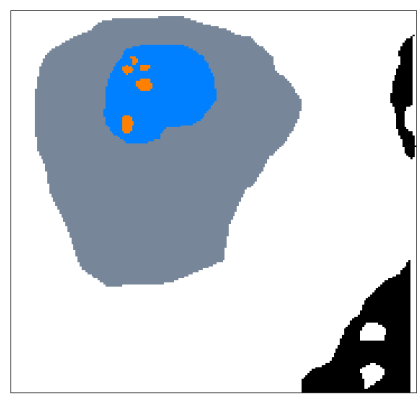

d)

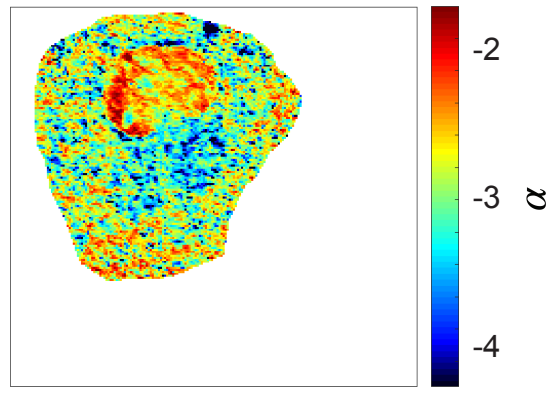

$-3 \gamma$

$\times 10^{19}$

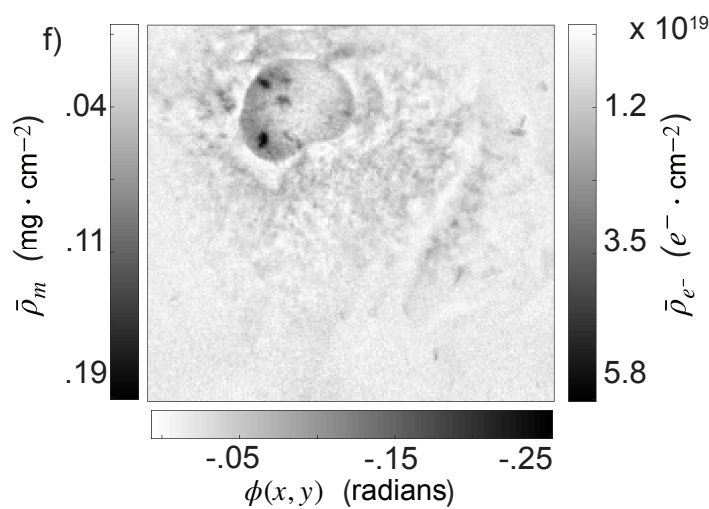

FiguRE 5.9: Additional example of a cell included in the study presented throughout chapter 5. (a) Dark field image for the $\left[q_{0}, q_{\max }\right]$ range. The color scale is adjusted for the minimum and maximum number of detected photons. The scale bar is $10 \mu \mathrm{m}$ and also applies to subfigures (b), (d), (e) and (f). (b) Manually selected regions of interest of the heterochromatin (orange), euchromatin (blue), cytoplasm (gray) and background (black). (c) Averaged, azimuthally integrated and background-corrected diffraction patterns are plotted against the absolute value of the scattering vector $q$. The red and black dashed lines are the fits using $I(q)=K q^{\alpha}+B$. Around $q \simeq 0.1$ $\mathrm{nm}^{-1}$, a kink becomes distinguishable between low and high $q$-values. The black vertical lines at $q=0.038 \mathrm{~nm}^{-1}$ and $q=0.867 \mathrm{~nm}^{-1}$ represent $q_{0}$ and $q_{\max }$, respectively. The vertical dashed lines represent $q_{\min }$ of the corresponding ROI. The solid green lines are $\propto q^{-4}$ and only serve as a visual aid to the overall $I(q)$ decay. (d) Map of the exponent derived by fitting $I(q)$ in the $\left[q_{0}, q_{\min }\right]$ range. (e) Map of the Porod constant $K$, derived by fitting $I(q)$ in the $\left[q_{\min }, q_{\max }\right]$ range. (f) Map of the reconstructed phase, projected mass density and projected electron density. 


\subsubsection{Momentum Transfer Comparison}

Figure 5.10 shows the accessed $q$-range of the holographic (red) and scanning SAXS (magenta, blue) imaging modalities. The data correspond to the cell shown in Figure 5.2. As the accessed $q$-range of each modality is different, the substitution of $\Delta \rho_{e^{-}}$into $K=2 \pi\left(\Delta \rho_{e^{-}}\right)^{2} S$ to quantify the interface area between the sample and surrounding environment, $S$, is not possible.

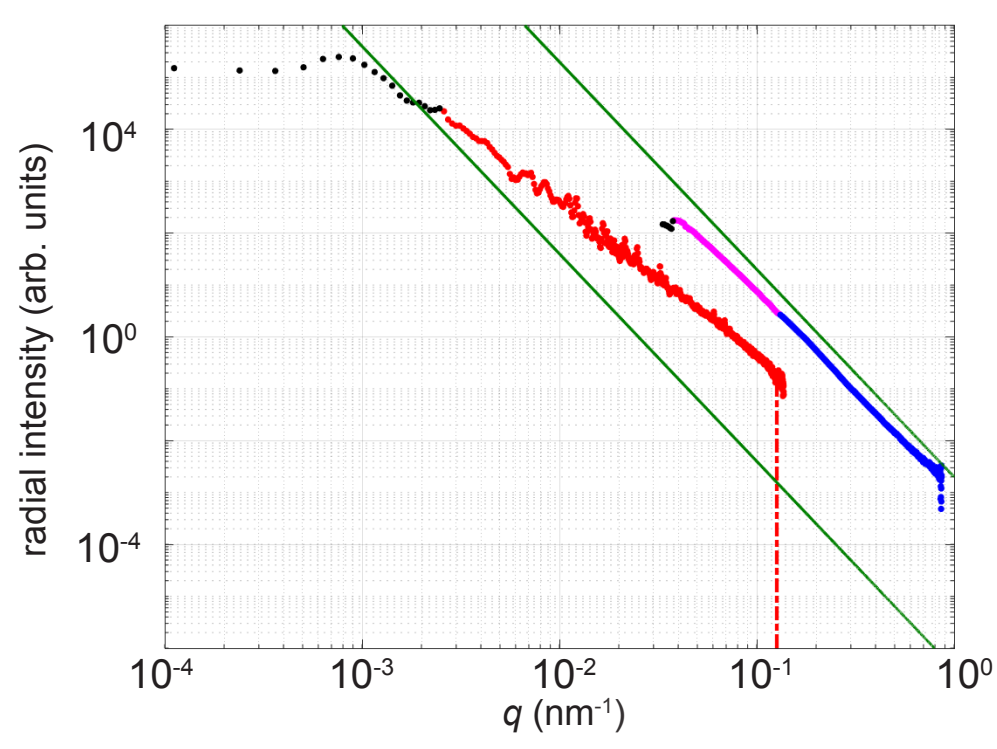

FiguRE 5.10: Comparison between the 1D averaged azimuthally integrated power spectral density (red) of a reconstructed phase map (single-distance, RAAR) and a typical radial intensity profile (magenta, blue) from scanning SAXS. The red, dashed vertical line at $q=0.119 \mathrm{~nm}^{-1}$ represents the transition from signal to noise and corresponds to a real-space resolution of $53 \mathrm{~nm}$. The typical fitting range considered for the Porod analysis is shown in blue. The green lines are proportional to $q^{-4}$ and serve as a visual aid to the overall power decay. Structures greater than $2.5 \mu \mathrm{m}$ in the holography PSD are omitted (black dots). Regions blocked by the beamstop in the scanning SAXS radial intensity profile are omitted (black dots).

\subsubsection{Cell Ensemble}

In total, data were collected and analyzed for $N=33$ cells. Histograms for each variable, including all analyzed data from all samples, are shown in Figure 5.5. Table 5.2 lists the median values of each variable for each region of interest. The values of $\alpha$ and $K$ are derived by fitting $I(q)$ profiles between $\left[q_{0}, q_{\min }\right]$ and $\left[q_{\min }, q_{\max }\right]$, respectively. 
TABle 5.2: Median values of the distributions shown in Figure 5.5.

\begin{tabular}{|c|c|c|c|c|c|}
\hline Region & $\alpha$ & $K$ (arb. units) & $-\phi(x, y)(\mathrm{rad})$ & $\bar{\rho}_{m}\left(\frac{\mathrm{mg}}{\mathrm{cm}^{2}}\right)$ & $\bar{\rho}_{e^{-}}\left(\frac{\mathrm{e}^{-}}{\mathrm{cm}^{2}}\right)$ \\
\hline \hline Heterochromatin & -2.68 & $5.63 \times 10^{-4}$ & 0.13 & 0.09 & $2.85 \times 10^{19}$ \\
Euchromatin & -2.78 & $1.64 \times 10^{-4}$ & 0.06 & 0.05 & $1.36 \times 10^{19}$ \\
Cytoplasm & -3.09 & $3.43 \times 10^{-5}$ & 0.02 & 0.01 & $4.03 \times 10^{18}$ \\
\hline
\end{tabular}




\section{Optical Fluorescence and X-ray Tomography Probe Cell Nuclei}

DNA within mammalian cell nuclei stores genetic information and is densely packed, spanning length scales from $2 \mathrm{~nm}$ to $1 \mu \mathrm{m}$, corresponding to double helices and individual chromosomes, respectively [5-7,9]. The structural architecture of packaged DNA on intermediate length scales fundamentally defines the function of the cell, thus its understanding is of critical importance. Understanding the structure of DNA is facilitated by imaging, and imaging structures on these length scales requires high resolution. X-rays $[122,123]$ and fluorescence $[120,121]$ are two ideal probes that satisfy this requirement.

$\mathrm{X}$-ray are sensitive to the electron density of a sample and are capable of imaging whole structures without the invasive need of slicing or staining. X-ray diffraction has shown that the structure of chromatin is based on repeating units of histone proteins which ultimately lead to the formation of chromatin fibers [150]. Chromatin fibers act as flexible, jointed chains which, when folded and coiled, form hierarchical structures. In fixed Drosophila melanogaster embryonic chromosomes, higher-ordered chromatin structures on length scales ranging from 80 to $160 \mathrm{~nm}$ have been visualized using X-rays [143]. Using soft X-ray tomography, the in vivo chromatin organization in differentiating mouse olfactory neurons has been investigated [151]. The authors observed distinct chromatin compartments form interconnected networks, which span the entire nucleus, remain intact during the nuclear organization process. The authors also observed heterochromatin to be $\sim 30 \%$ more compact than euchromatin. In the hard X-ray regime, ptychography has been combined with tomography to 3D image lyophilized Deinococcus radiodurans [37]. The authors identified dense, globular regions to be rich in DNA with a typical mass density of $1.6 \mathrm{~g} \cdot \mathrm{cm}^{-3}$. X-ray ptychography has also been combined with X-ray fluorescence to probe whole, unlabeled Chlamydomonas reinhardtii in 3D [152]. The 
authors identified various organelles and found a strong correlation between electrondense bodies and regions with strong phosphorous and calcium signals. This correlation lead the authors to associate these regions with acidocalcisomes, acidic organelles which serve as storage sites for phosphorous and calcium [153].

Imaging of specifically-labeled cellular components is possible using visible-light fluorescence microscopy. In particular, using 3D STED microscopy, mitotic chromosomes in HeLa cells were observed to have a grained structure as well as small loops of DNA [40]. In Escherichia coli, this same imaging method enabled the 3D radial positioning of DNA in proximity to the bacterial plasma membrane to be visualized [40]. Joining visible-light fluorescence microscopy with X-ray microscopy combines the strength of both methods. The combination of tomographic coherent X-ray diffraction imaging and $2 \mathrm{D}$ visible-light fluorescence microscopy has been applied to image cell nuclei in mouse fibroblasts [39]. The diffraction imaging enabled the authors to explicitly visualize and quantify 3D nuclear substructures at several tens of nanometer resolution. The authors determined the average electron density of the nucleus and nucleolus to be $440 \mathrm{e}^{-} \cdot \mathrm{nm}^{-3}$ and $620 \mathrm{e}^{-} \cdot \mathrm{nm}^{-3}$, respectively. The authors also attributed specific regions within the nucleus to be heterochromatin by associating them with bright spots observed in the fluorescent data. Within heart tissue cells, the structure of the actin network has been probed using a combination of X-ray holography, scanning SAXS and 2D STED microscopy [93]. The authors concluded the orientations of the actin filaments observed in the holography and STED images are correlated with the principle directions observed in the local SAXS patterns.

The combination of visible-light fluorescence and X-ray holographic tomography is particularly fruitful as the collected signal originates from different sources: the fluorescent signal of specified structures in the confocal or STED images, and the total electron density within the sample. In this respect, the data sets complement each other as each offers a quantitative contrast mechanism sensitive to different entities within the ensemble of the biomolecular constituents at the single-cell level.

An aim of this thesis is to extended these successful approaches by combining visible-light fluorescence microscopy with X-ray holographic tomography to probe whole cell nuclei of both lyophilized and chemically-fixed hydrated 3T3 fibroblasts. On the one hand, lyophilized samples are simplistic to handle but do not accurately reflect physiological conditions. On the other hand, chemically-fixed hydrated cells more-accurately represent physiological conditions but can be more difficult to handle and image with X-rays as a result of the low electron density contrast between the sample and environment, and the motility of free radicals produced by water radiolysis which leads to severe radiation damage [154]. For simplicity, lyophilized samples are first considered within this work, 
followed by hydrated samples. This chapter is divided into two sections: the fluorescence data of a lyophilized and hydrated sample are first compared, followed by a separate comparison of tomography data corresponding to a lyophilized and hydrated sample.

\subsection{Sample Preparation and Experimental Setups: La- beled Lyophilized and Hydrated Nuclei}

The labeling of both lyophilized and chemically-fixed 3T3 fibrobalsts is performed using the Click-iT EdU Alexa Fluor 647 (C10340, Thermo Fisher Scientific) imaging kit. Detection of the fluorophore is based on a "Click" [83] reaction, a copper-catalyzed covalent reaction between an azide and alkyne via a process called bioconjugation. In the Click-iT EdU cell proliferation assay EdU (5-ethynyl-2'-deoxyuridine), a nucleoside analog of thymidine, is incorporated into DNA during DNA synthesis. In this application, the EdU contains the alkyne and the Alexa Fluor 647 dye contains the azide. The dye has a peak excitation and emission wavelength of $\lambda_{\mathrm{ex}}=650 \mathrm{~nm}$ and $\lambda_{\mathrm{em}}=668 \mathrm{~nm}$, respectively. The protocol used for labeling is found in Appendix A.2.

\section{Lyophilized Fibroblasts}

All fluorescently labeled lyophilized fibroblasts are adhered to $\mathrm{Si}_{3} \mathrm{~N}_{4}$ windows (see section 4.1.1). A window glued to a brass pin and mounted in a sample holder is shown in Figure 6.1a. The fluorescence data corresponding to the lyophilized sample shown in this chapter is recorded using a custom-built STED microscope (see section 4.6) coupled into the GINIX endstation of the P10 beamline at DESY (Hamburg, Germany) during run 61. Hereafter, this microscope will be referred to as the "IRP (Institute für Röntgenphysik) STED". To preserve the integrity of the fluorophores, fluorescent recordings are performed prior to the X-ray measurements. Figure $6.1 \mathrm{~b}$ shows the mounted $\mathrm{Si}_{3} \mathrm{~N}_{4}$ window in the focal plane of the STED objective. Typical experimental parameters used to acquire fluorescence images using the IRP STED are listed in Table 6.1.

\section{Chemically-Fixed Hydrated Fibroblasts}

The chemically-fixed cells are adhered to a $1 \mu \mathrm{m}$-thick, $3 \mathrm{~cm}$ diamteter polypropylene foil and are encapsulated in a polypropylene chamber with $300 \mu \mathrm{L}$ of PBS, (see section 4.1.1). The PBS layer is approximately $425 \mu \mathrm{m}$ thick. An example of a single polypropylene foil with cells adhered to it is shown in Figure 6.1c. The imaging of hydrated samples occurred after run 61 ended. Consequently, image acquisition is not restricted to the 
IRP STED. Although the IRP STED is capable of imaging a polypropylene chamber, it is equipped with a low numerical aperture objective $(\mathrm{NA}=0.95)$ when compared to a typical inverted STED microscope (NA > 1). Thus, in order to optimize the image quality, images of hydrated samples are not acquired with the IRP STED but rather a STED microscope engineered by Dr. Francesco Rocca of the Laser Laboratory (Göttingen, Germany) coupled into an IX83 inverted microscope (Olympus) equipped with a higher numerical aperture objective $(\mathrm{NA}=1.2)$. Hereafter, this microscope will be referred to as the "laser lab STED" A polypropylene chamber on the sample stage of the laser lab STED is shown in Figure 6.1d.

TABLE 6.1: Experimental parameters used to acquire confocal and STED images of lyophilized cells adhered to $\mathrm{Si}_{3} \mathrm{~N}_{4}$ windows and chemically-fixed hydrated cells adhered to polypropylene rings. Lyophilized samples are imaged with the IRP STED, and chemically-fixed hydrated samples are imaged with the laser lab STED.

\begin{tabular}{|c|c|c|}
\hline Parameter & Lyophilized & Chemically-fixed hydrated \\
\hline \hline Beamtime run & 71 & None \\
\hline Fluorophore & Alexa Fluor 647 & Alexa Fluor 647 \\
\hline Objective magnification & $40 \times$ & $60 \times$ \\
\hline Objective immersion & Air & Water \\
\hline Numerical aperture & 0.95 & 1.2 \\
\hline$\lambda_{\text {excitation }(\mathrm{nm})}$ & 632 & 640 \\
\hline$\lambda_{\text {depletion }}(\mathrm{nm})$ & 775 & 775 \\
\hline Excitation power $(\mu \mathrm{W})$ & 120 & 0.7 \\
\hline Depletion power $(\mathrm{mW})$ & 120 & 90 \\
\hline Scan area $\left(\mu \mathrm{m}^{2}\right)$ & 30 & 16 \\
\hline Lateral step size $\left(\mathrm{nm}{ }^{2}\right)$ & $100 \times 100$ & $20 \times 20$ \\
\hline Axial step size $\left(\mathrm{nm}^{2}\right)$ & 250 & 500 \\
\hline Dwell time $\left(\mathrm{ms}^{2}\right.$ & 0.29 & 0.02 \\
\hline
\end{tabular}



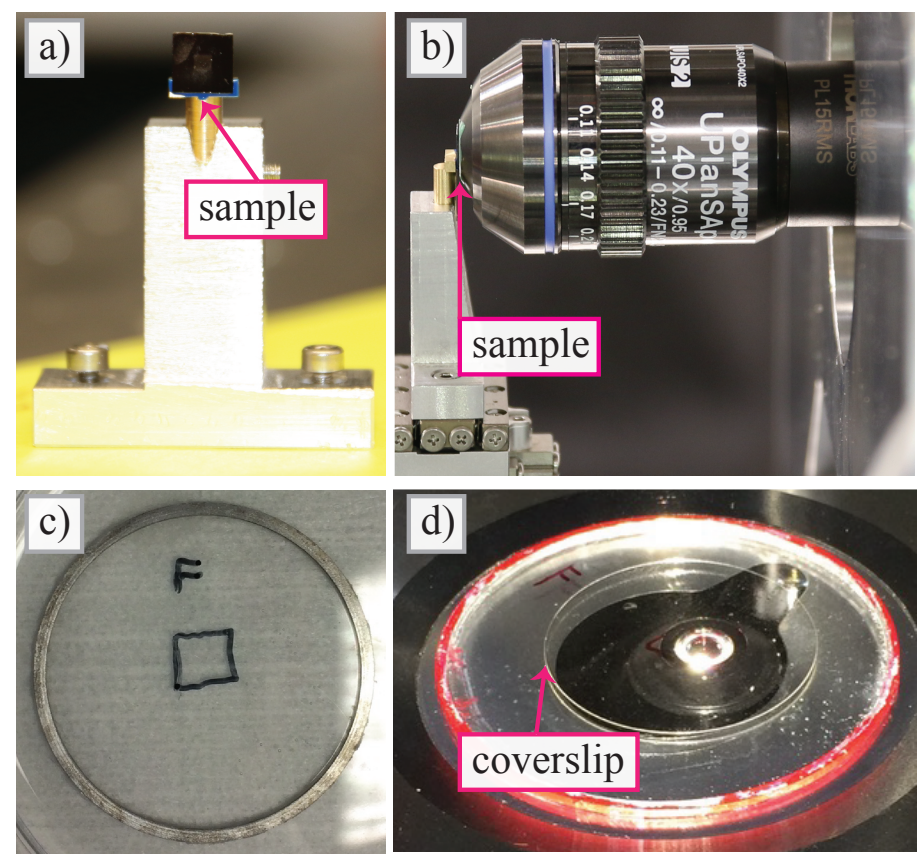

Figure 6.1: a) $\mathrm{A} \mathrm{Si}_{3} \mathrm{~N}_{4}$ window glued to a brass pin and mounted on the sample tower is positioned b) in the focal plane of the IRP STED objective. To account for the $170 \mu \mathrm{m}$ correction of the objective, a coverslip is glued to the onto the objective (not shown). c) A single $1 \mu \mathrm{m}$-thick polypropylene foil with cells adhered to it. The black square and "F" are used as references when navigating the sample. d) A polypropylene chamber on the sample stage of the laser lab STED microscope. The chamber is held together by red nail polish. To account for the $170 \mu \mathrm{m}$ correction of the objective, a coverslip is placed underneath the chamber.

\subsection{Results and Discussion: Labeled Lyophilized and Hy- drated Nuclei}

Figure 6.2a shows an overlay of an epifluorescent and visible-light phase contrast image of a lyophilized fibroblast adhered on a $\mathrm{Si}_{3} \mathrm{~N}_{4}$ window. Both images are acquired using a Zeiss AXIO Observer Z.1 microscope equipped with a $40 \times$ phase contrast objective. Corresponding confocal and STED images, both acquired using the IRP STED microscope, are shown in Figure $6.2 \mathrm{~b}$ and c, respectively. Furthermore, Figure 6.2d shows a chemically-fixed hydrated fibroblast adhered to a $1 \mu \mathrm{m}$-thick polypropylene foil. Corresponding confocal and STED images, both acquired using the laser lab STED microscope, are shown in Figure 6.2e and f, respectively. 

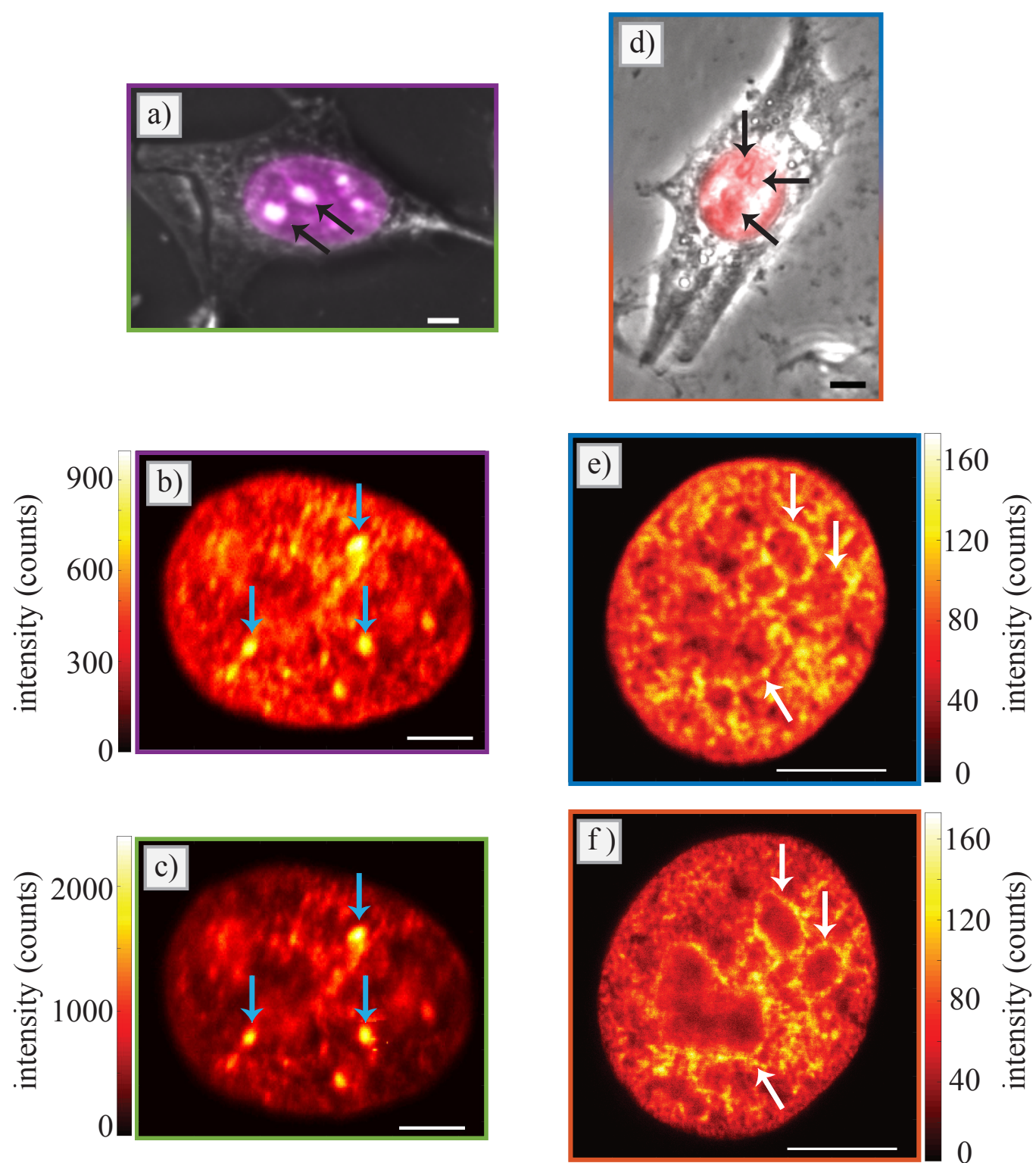

Figure 6.2: a) Epifluorescent image overlaid on a visible-light phase contrast image of a a) lyophilized fibroblast and d) hydrated fibroblast in the chemically-fixed state.

b) Confocal and c) STED images of the lyophilized cell shown in a). e) Confocal and

f) STED images of the chemically-fixed cell shown in d). The perimeter color of each image corresponds to the legend shown in Figure 6.5. All scale bars are $5 \mu \mathrm{m}$.

As indicated by the black arrows in both Figure 6.2a and d, there exist several globular structures. In the lyophilized sample, only two of the possible four globular structures are explicitly identified. Similar structures have also been observed using visible-light phase contrast microscopy and have been identified as nucleoli [134, 135]. Nucleoli are responsible for rRNA synthesis [136] and are composed of several hundred types of proteins [134], as well as rDNA and RNA [137-139]. We hypothesize the globular structures observed here are also nucleoli. When the nucleus is fluorescently labeled with 
DNA dyes, nucleoli appear as dark regions surrounded by brighter nuclear chromatin [155]. Interestingly, the presence of nucleoli is not readily observed in the confocal (Figure 6.2b) and STED (Figure 6.2c) images of the lyophilized sample. Instead, bright regions are observed, as indicated by blue arrows, which correspond to regions of high DNA content and may possibly be chromosomes. These bright regions are observed in neither the confocal (Figure 6.2e) nor STED (Figure 6.2f) images of the hydrated sample. Rather, the nucleoli regions are observed, as indicated by the white arrows in both subfigures (e and f). Another noticeable difference when comparing the lyophilized and chemically-fixed images is the photon counts: The fluorescence images of the lyophilized sample have a factor of approximately $6-12 \times$ higher counts than the hydrated sample. This increase is associated with the fluorescence signal propagating through air rather than water.

The confocal and STED images of Figure 6.2 are only single focal planes of 3D volumes. For a more accurate description of the nuclei, confocal stacks through the nuclei are recorded. The stacks are rendered by acquiring images along the axial direction of the objective using the parameters listed in Table 6.1. Figure 6.3a and b show confocal stacks of the lyophilized and chemically-fixed hydrated nucleus, respectively. All scanning locations with fewer than 10 photon counts have been rendered transparent. The color scale of each stack ranges from 10 to the maximum number of detected counts of the respective stack. 
a)

b)

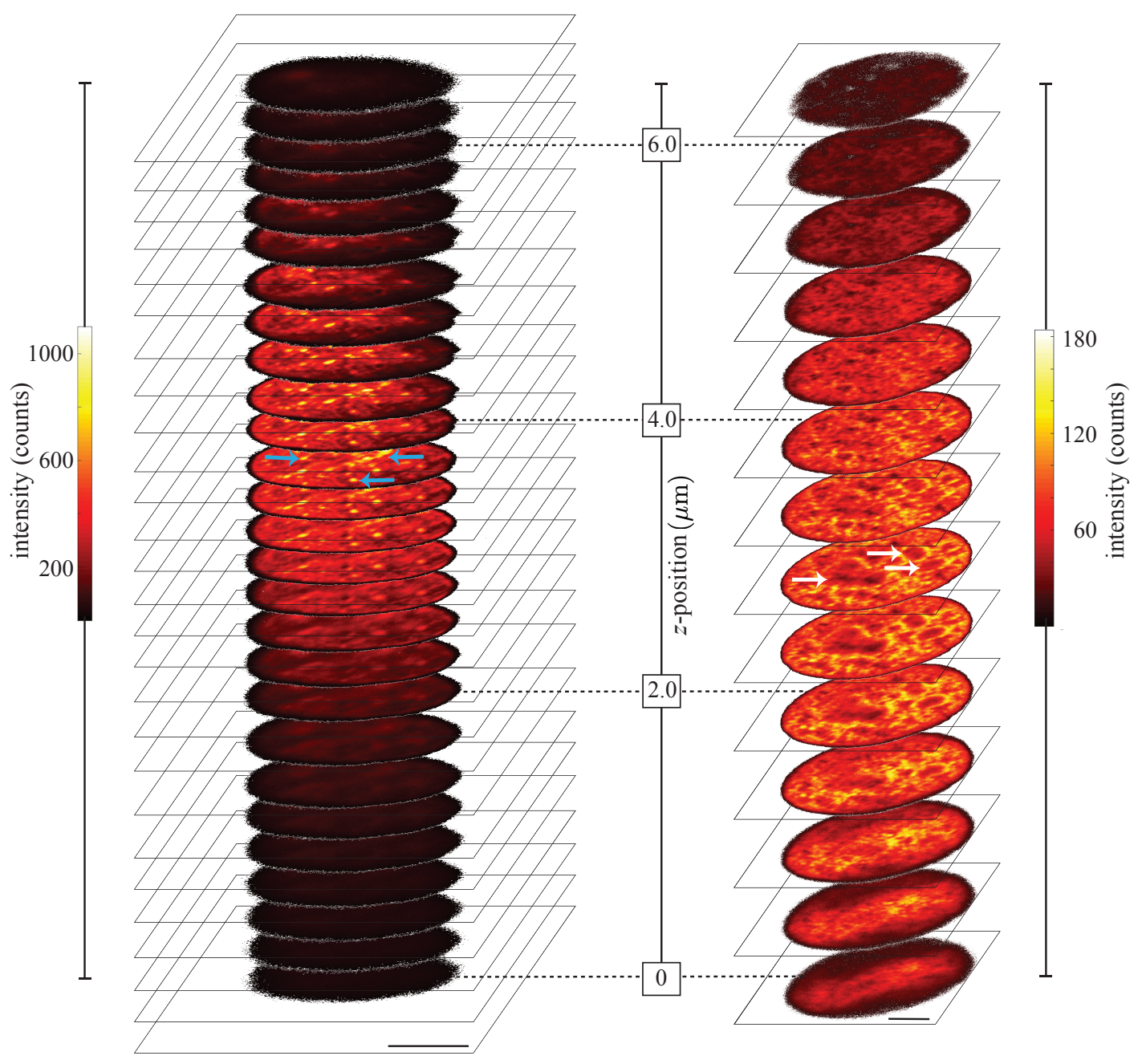

Figure 6.3: a) Stack of confocal images through the lyophilized nucleus shown in Figure 6.2 a. A total volume of $30 \times 30 \times 6.5 \mathrm{\mu m}^{3}$ is imaged using the parameters listed in Table 6.1. Images are recorded with the IRP STED (section 4.6). The blue arrows indicate regions of high DNA concentration, possibly corresponding to chromosome territories. b) Stack of confocal images through the chemically-fixed hydrated nucleus shown in Figure $6.2 \mathrm{~d}$. A total volume of $16 \times 16 \times 6.5 \mathrm{\mu m}^{3}$ is imaged using the parameters listed in Table 6.1. Images are recorded with the laser lab STED. The white arrows indicate globular regions, possibly corresponding to nucleoli. Both scale bars are $5 \mu \mathrm{m}$.

From visual inspection of Figure 6.3a, the relative increase in intensity indicates a majority of the DNA within the lyophilized nucleus is concentrated in the range of approximately 2 to $5 \mu \mathrm{m}$ in height. The fluorescence intensity decreases in the ranges of approximately 0 to $2 \mu \mathrm{m}$ and 5 to $6.5 \mu \mathrm{m}$. In comparison, the fluorescence intensity of chemically-fixed hydrated nucleus (Figure $6.3 \mathrm{~b}$ ) is approximately constant throughout the volume. In the lyophilized nucleus, the bright regions observed in Figure $6.2 \mathrm{~b}, \mathrm{c}$ possibly corresponding to chromosome territories, are observed in the stack, as indicated by the blue arrows. However, these regions are not observed throughout the 
entire volume but for only for frames located approximately 3 to $4.5 \mu \mathrm{m}$ in $z$, suggesting these territories are around $1.5 \mu \mathrm{m}$ in height. Furthermore, the dark regions observed in the chemically-fixed hydrated nucleus shown in Figure 6.2e,f, possibly corresponding to nucleoli, are also observed in the confocal stack, as indicated by the white arrows in Figure 6.3b. These dark regions are only observed for frames located approximately 1 to $4 \mu \mathrm{m}$ in $z$, suggesting the nucleoli are about $3 \mu \mathrm{m}$ in height. Using transmission electron microscopy, the diameter of nucleoli have been approximated to be $2 \mu \mathrm{m}$ [134].

To compare how the entire volume of the confocal stacks relate to the single frames shown in Figure 6.2, the average and maximum intensities projected through each stack are calculated, as shown in Figure 6.4. The average intensity projection maps the average intensity value recorded at a specific scan point in all frames for all scanning locations. Similarly, the maximum intensity projection maps the maximum intensity value recorded at a specific scan point in all frames for all scanning locations. 

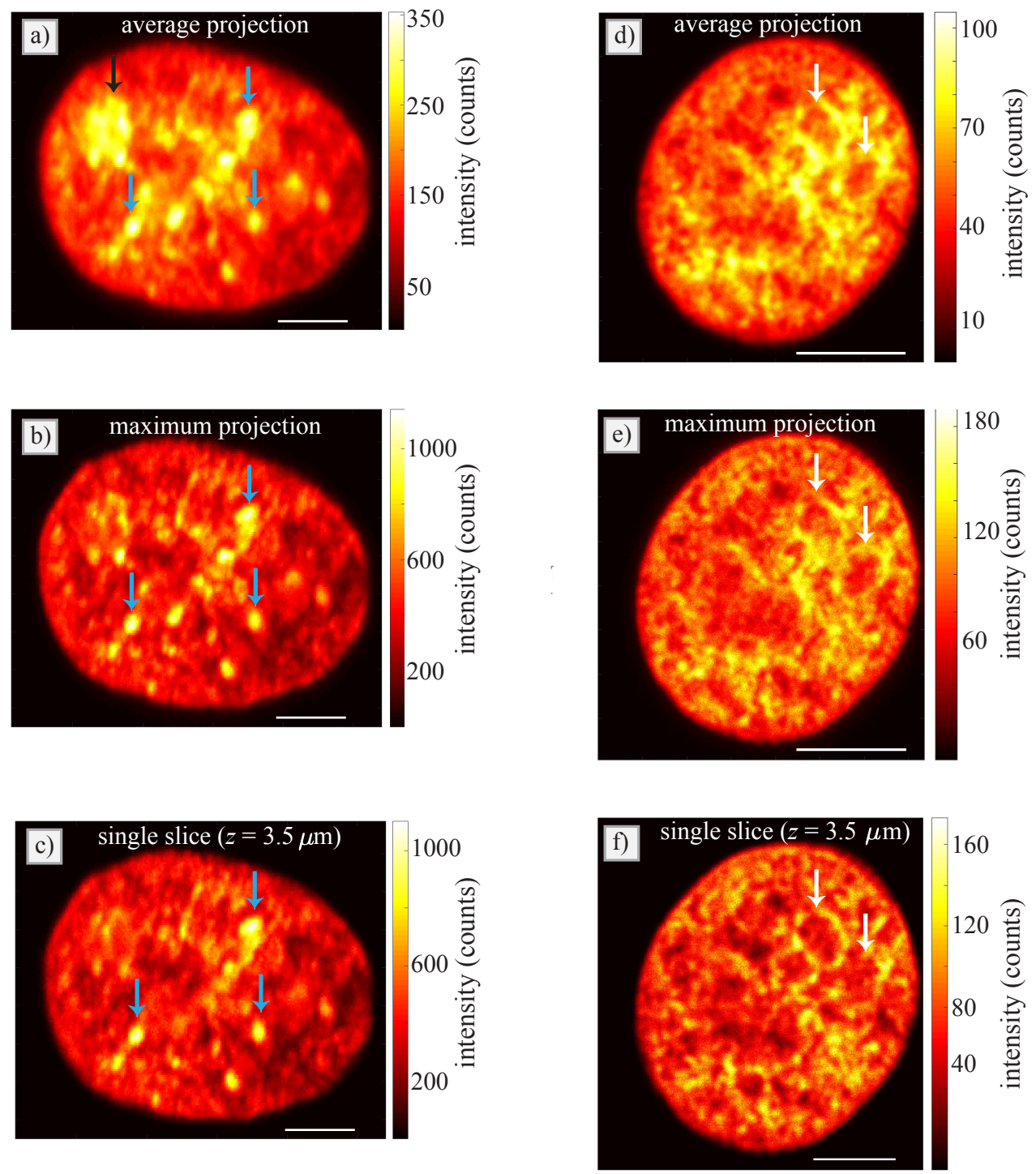

Figure 6.4: a) Average and b) maximum intensity projections through the lyophilized confocal stack set shown in Figure 6.3a. c) Single frame of from the stack. The black arrow in a) indicates a region throughout the entire nuclear volume where DNA is concentrated. The blue arrows in a), b) and c) indicate regions of high DNA concentration, possibly corresponding to chromosome territories. d) Average and e) maximum intensity projections through the chemically-fixed hydrated confocal stack shown in Figure $6.3 \mathrm{~b}$. f) Single frame from the stack. The white arrows indicate globular regions, possibly corresponding to nucleoli. All scale bars are $5 \mu \mathrm{m}$.

With respect to the lyophilized sample, the bright spots associated with possible chromosome territories are observed in both the average (Figure 6.4a) and maximum intensity projections (Figure 6.4b), as indicated by the blue arrows. Particularly, the average intensity projection suggest there is a region near the top left of the nucleus, as indicated by the black arrow, which has DNA concentrated within it throughout the entire height 
of the nucleus. This region is not pronounced in either the maximum intensity projection or single frame (Figure 6.4c). Concerning the chemically-fixed hydrated sample, the dark regions associated with nucleoli in are pronounced in both the average (Figure 6.4d) and maximum (Figure 6.4e) intensity projections.

\section{Fluorescence Resolution Approximation}

To quantitatively compare the difference in image quality between the confocal and STED images acquired using the IRP STED and laser lab STED, the resolution, i.e., the smallest resolvable feature, of each micrograph shown in Figure 6.2 is approximated. Towards this end, the resolution is estimated using the power spectral density (PSD), which is a measure of signal strength as a function of spatial frequency. By determining the signal-to-noise transition, the resolution can be approximated.

For each fluorescent image shown in Figure 6.2, its Fourier transform is first calculated and subsequently azimuthally averaged to obtain a 1D PSD. Figure 6.5 shows the 1D PSDs corresponding to the lyophilized confocal (purple) and STED (green) images, which are both acquired using the IRP STED microscope. Also shown in Figure 6.5 are the 1D PSDs corresponding to the chemically-fixed hydrated confocal (blue) and STED (orange) images, which are both acquired using the laser lab STED microscope. To evaluate a 1D PSD as a function of the scattering vector $q$, all spatial frequencies $\nu$ belonging to it are transformed into $q$-equivalents by $q=2 \pi \nu$. The signal-to-noise transition is determined by fitting the slope of a PSD with a power-law function in the form of $I(q)=a q^{b}+c$, as shown by the bold, black lines in Figure 6.5. The cross-over point $q_{\text {cross }}$ is derived from $a q_{\text {cross }}=b g$, where $b g$ denotes the constant background level found at high $q$ values. In Figure 6.5, the bold, grey lines represent the fits of the background levels. 


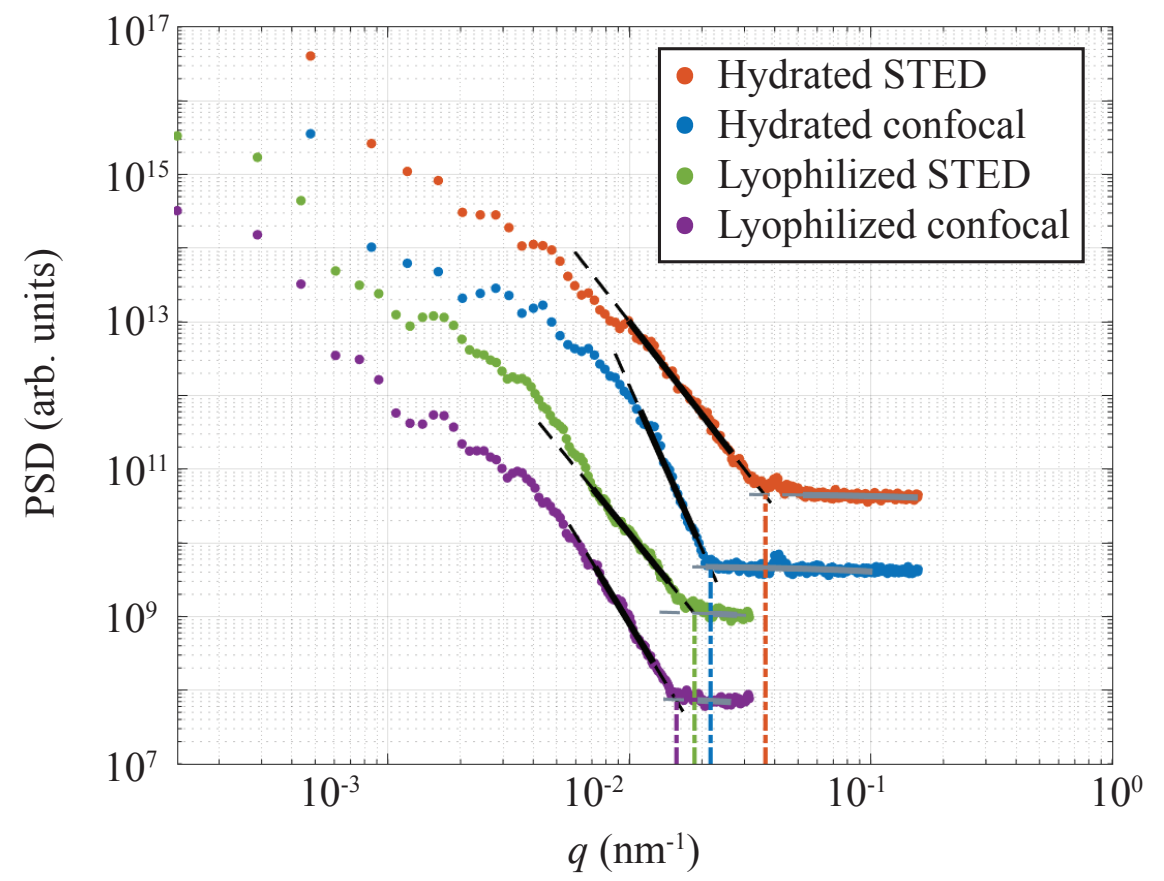

FIguRE 6.5: The power spectral densities of the confocal and STED images belonging to the lyophilized and chemically-fixed fibroblasts (Figure 6.2) are azimuthally averaged and plotted against the magnitude of the scattering vector $q$. The color code of the legend corresponds to the color of each border shown in Figure 6.2. Images of the hydrated sample are recorded with the laser lab STED, and images of the lyophilzed sample are recorded with the IRP STED. The cross-over values $q_{\text {cross }}$ from signal to noise are represented by the dot-dashed vertical lines and are proportional to the real space resolution $d$ by $d=2 \pi / q_{\text {cross }}$. The bold black and grey lines represents the fits to the signal and background, respectively. The dashed black and grey lines serve as a visual aid to the fits of the signal. Intensity values are shifted for better visualization. The cross-over and resolution values are listed in Table 6.2.

The scattering vector $q$ is proportional to the real space resolution $d$ by $d=2 \pi / q$. The real space resolution obtained from each imaging modality is presented in Table 6.2.

TABLE 6.2: Resolution, i.e., the smallest resolvable feature, approximation for the confocal and STED images shown in Figure 6.2. The $q_{\text {cross }}$ values correspond to the cross-over points denoted by the vertical, dot-dashed lines in Figure 6.5.

\begin{tabular}{|c|c|c|c|}
\hline Image & Microscope & $q\left(\mathrm{~nm}^{-1}\right)$ & $d(\mathrm{~nm})$ \\
\hline \hline Hydrated STED & Laser lab STED & 0.036 & 175 \\
Hydrated confocal & Laser lab STED & 0.022 & 286 \\
Lyophilized STED & IRP STED & 0.019 & 330 \\
Lyophilized confocal & IRP STED & 0.016 & 392 \\
\hline
\end{tabular}

As expected, the results indicate an improvement in resolution from confocal to STED 
imaging for both the IRP STED and laser lab STED microscopes. Overall, the hydrated confocal (Figure 6.2e) and hydrated STED (Figure 6.2f) images acquired with the laser lab STED yield higher resolution than the lyophilized confocal (Figure 6.2b) and lyophilized STED (Figure 6.2c) images acquired with the IRP STED. This increase in resolution is attributed to both the higher numerical aperture and immersion medium of the laser lab STED objective $\left(\mathrm{NA}_{\text {laser lab }}=1.2\right.$ vs. $\mathrm{NA}_{\mathrm{IRP}}=0.95$; water vs. air $)$.

\subsection{Sample Preparation and Experimental Setups: To- mography Recordings}

Concerning the X-ray tomography recordings, samples are imaged using the GINIX endstation at the P10 beamline, as introduced in section 4.5.1. All tomographic recordings use the parameters listed in Table 4.3. In total, 1500 holographic projections are recorded over an angular range of $180^{\circ}$. Thus, projection is recorded every $0.12^{\circ}$. The "missing wedge" (see section 4.5.2) defines the angular range where the sample substrate is parallel to the X-ray beam, resulting in a loss of signal from the sample.

\subsection{Results and Discussion: Tomography Recordings}

The measured holograms of the holographic tomography measurements are reconstructed using the CTF algorithm (see section 4.4.1). Reconstruction yields 2D phase maps of the phase shift projected along the propagation direction of the X-rays. Various phase maps recorded at different rotation angles of the lyophilized sample shown in Figure 6.2a are shown in Figure 6.6. The reconstructed phase is proportional to the projected mass density (mass density per area, see eq. 4.19). Thus, the projected mass density is also shown in Figure 6.6. 

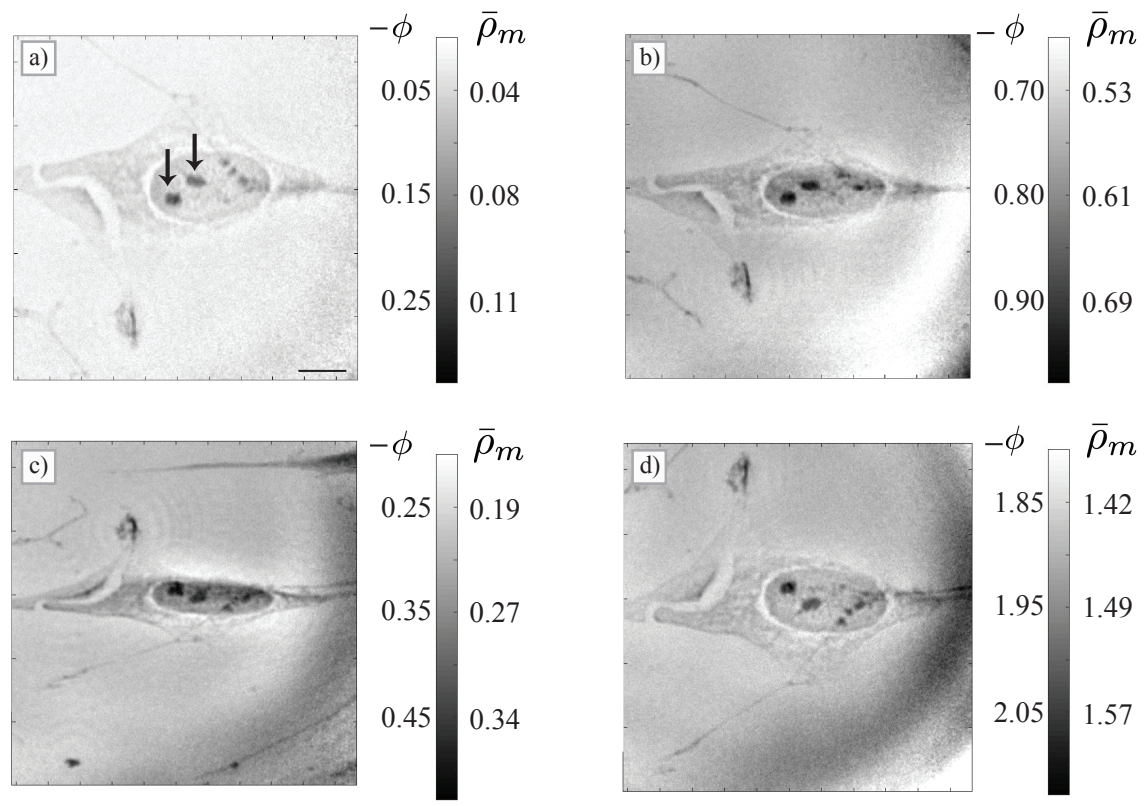

FiguRE 6.6: Reconstructed phase and projected mass density maps recorded at different angles of the lyophilized cell shown in Figure 6.2a, with units of radians and $\mathrm{mg} \cdot \mathrm{cm}^{-2}$, respectively. The projections are recorded at a) $0^{\circ}$, b) $47.87^{\circ}$, c) $119.88^{\circ}$ and d) $155.87^{\circ}$. The "missing wedge" for this data set is $44^{\circ}$. The scale bar in a) is $10 \mu \mathrm{m}$ and applies to all subfigures.

The structures observed in Figure 6.2a, suspected to be nucleoli, are also observed in Figure 6.6, as indicated by the black arrows in subfigure a). Of all structures in the projected mass density maps, these regions have the highest projected mass density. This suggest the regions are nucleoli, as nucleoli are densely packed with proteins and would thus yield the highest mass density. The 3D volume is reconstructed using the filtered back projection, as introduced in section 4.5. Visualization of the $3 \mathrm{D}$ volume is done using the software Avizo Lite (version 9.2.0., Thermo Fisher Scientific). Orthogonal frames showing the 3D structure of the nucleus are shown in Figure 6.7. In each subfigure, the nucleoli structures are indicated by two black arrows.
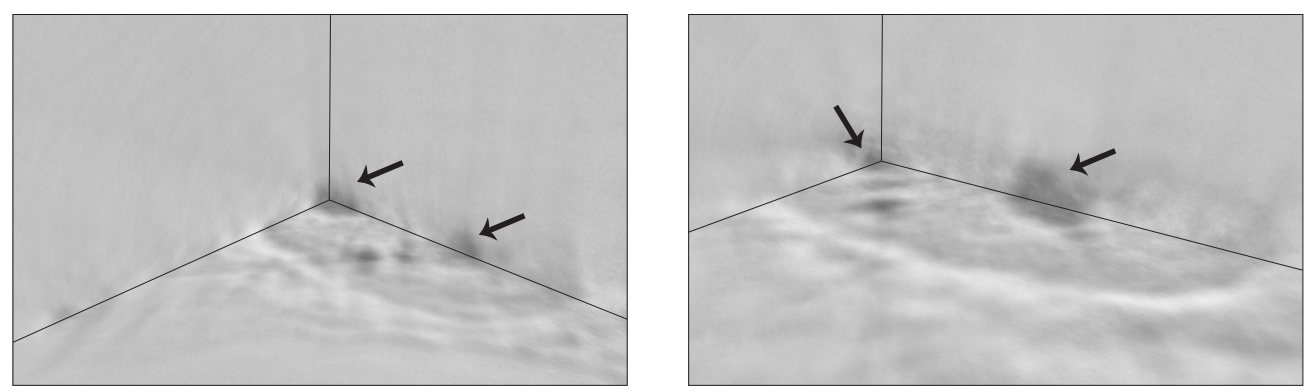

Figure 6.7: 3D visualization of the lyophilized cell shown in Figure 6.2a using the software Avizo Lite. The nucleoli structures are indicated by black arrows. 
With respect to the chemically-fixed hydrated sample shown in Figure 6.2b, no X-ray tomography data were recorded. However, data were collected for a different cell on the same substrate. Various reconstructed phase maps recorded at different rotation angles are shown in Figure 6.8.
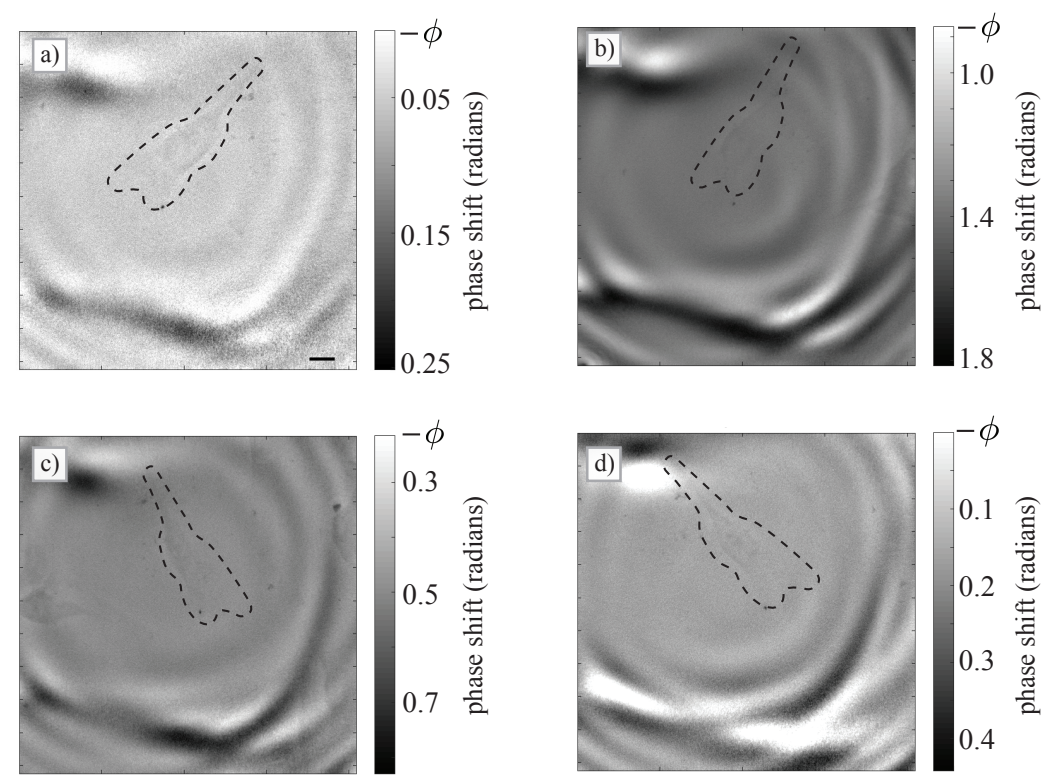

Figure 6.8: Reconstructed phase maps recorded at a) $0^{\circ}$, b) $47.87^{\circ}$, c) $119.88^{\circ}$ and d) $155.87^{\circ}$. For visual aid, the body of the cell in each subfigure is loosely outlined by a black, dotted line. The "missing wedge" for this data set is $18^{\circ}$. The scale bar in a) is $10 \mu \mathrm{m}$ and applies to all subfigures. This cell is not related to the hydrated cell shown in Figure 6.2b.

As can be seen throughout Figure 6.8, the contrast very poor. This is attributed to the attenuation of the beam due in the liquid environment as well as the poor electron density contrast between the sample and surrounding environment.

In this chapter, the applications of X-ray phase contrast tomography and visible-light fluorescence microscopy are applied to visualize both lyophilized and chemically-fixed hydrated cell nuclei. The fluorescence data of the lyophilized sample indicates regions of high DNA concentration, which may correspond to chromosome territories. These regions are not observed in the fluorescence data of the hydrated sample. In comparison dark, globular regions, possibly corresponding to nucleoli, are observed. Interestingly, similar globular regions are observed in the tomography data, but not in the fluorescence data, of the lyophilized cell. As highlighted by the collective data of the lyophilized sample, only by combining the two imaging modalities it is possible to achieve a more accurate understanding of the structure of the nucleus. 



\section{Summary and Conclusion}

The genetic information necessary to develop and operate all living organisms is encoded in DNA. Indeed, the hierarchical structure of DNA fundamentally defines the function of a cell. Despite the critical importance of DNA, a complete understanding of its hierarchical structure is lacking due to its remarkable complexity. The central aim of this thesis is to therefore probe the hierarchical structure and spatial distribution of DNA within whole, intact cell nuclei of NIH-3T3 fibroblasts. To do so, a combination of imaging techniques is employed: scanning small-angle X-ray scattering (SAXS), X-ray in-line holography, $\mathrm{X}$-ray phase contrast tomography and super-resolution visible-light fluorescence microscopy. X-rays are sensitive to the electron density of a sample and can image whole structures without the need of sectioning. Visible-light fluorescence offers the unique capability to image specified structures within a sample. Both X-ray and visible-light fluorescence microscopy are able to image structures at the sub-cellular level.

By combining the aforementioned imaging techniques, structural information obtained in Fourier space is spatially correlated with structural information in real space. Analysis of X-ray dark field images, power-law fits of radial intensity profiles and quantitative phase contrast imaging, yields access to the length scales of the scatterers, aggregation and morphology and the projected electron and mass densities of the nuclear material. Separate dark field representations for different momentum transfer ranges reveal nuclear regions containing nucleoli, heterochromatin and euchromatin. As revealed by the analysis of radial intensity profiles, the scatterers within these three regions all share similar morphology, but differ in aggregation. In particular, distinct aggregation regions dominated by heterochromatin and some, but not all, nucleoli are observed. X-ray 
holography is able to clearly distinguish all nucleoli by quantitative electron and mass density analysis. Furthermore, X-ray phase contrast tomography and visible-light fluorescence are able to distinguish the nucleoli. The fluorescence data suggest the nucleoli are approximately $3 \mu \mathrm{m}$ in diameter, which is in good agreement with previous findings [134]. The nucleoli, heterochromatin and euchromatin are not all simultaneously observed in all representation schemes: euchromatin is only pronounced in the dark field, whereas heterochromatin is observed in both the dark field and aggregation representations. Nucleoli are observed in these two representations, as well as the holography, tomography and visible-light fluorescence. Distinct regions of DNA territories approximately $1.5 \mathrm{\mu m}$ in height, possibly corresponding to chromosome territories, are only observed in the visible-light fluorescence data. From a biological point of view, the data show that nucleoli are the densest structures in the nucleus and scatter mostly on length scales up to $60 \mathrm{~nm}$, indicating the existence of sub-structures in this size range, possibly proteins. While the density of heterochromatin and euchromatin is similar and lower than for nucleoli, as revealed by X-ray holography, heterochromatin mostly scatters on length scales above $35 \mathrm{~nm}$ and euchromatin scatters on all probed length scales. The aggregation maps reveal that compared to euchromatin, heterochromatin and nucleoli are more aggregated. In agreement with literature [135, 142, 143], heterochromatin is found to be located near the periphery of the nucleus and euchromatin to fill all other regions that are not occupied by heterochromatin or nucleoli.

In conclusion, scanning SAXS, X-ray in-line holography, X-ray phase contrast tomography and visible-light fluorescence are shown to be crucial tools for investigating and characterizing in vitro structures within cell nuclei. This thesis demonstrates that only by combining information obtained throughout the various imaging modalities can important nuclear structures, e.g., nucleoli, heterochromatin and euchromatin, be identified and characterized according to size, density and aggregation state. The combined imaging approaches demonstrated in this thesis can be applied to investigate and characterize a large number of biologically complex systems, ranging from the sub-cellular to tissue levels. 
Appendix

A

Protocols

\section{A.1 Grid Plunging}

The following section describes the protocol used when grid plunging biological cells adhered to $\mathrm{Si}_{3} \mathrm{~N}_{4}$ windows. This protocol supplements section 4.1.3. Within the scope of this thesis, the aim of the grid plunging process is to vitrify liquid within a biological cell, thus creating an amorphous layer which preserves its structural integrity. After grid plunging, the lyophilization process facilitates the sublimation of all volatile constituents, thus improving the electron density contrast when imaging with hard X-rays. An overview of the Leica EM GP grid plunger is shown in Figure A.1a.

\section{Preparation}

A $\mathrm{Si}_{3} \mathrm{~N}_{4}$ window is stored in a cryovial (VWR product 4791208). To prepare a cryovial, holes must be drilled into its cap; this allows for nitrogen to escape when it transitions from the liquid to gaseous phase. A $1 \mathrm{~mL}$ pipette tip is placed into the cryovial to secure the window and to improve sample handling. M4 nuts are cleaned in a sonication bath of ethanol and then placed at the base of the pipette tip. The nuts serve two purposes: to ensure the pipette tip does not float away when the vial is eventually stored in $\mathrm{LN}_{2}$, and their heat capacity helps keep the cryovial cool. A comparison between an unprepared and prepared cryovial is shown in Figure A.1b. 


\section{Plunging Process}

To ensure the cells do not begin to dry out prematurely, and thus have their structural integrity damaged, they are kept in a humidity-controlled chamber. The temperature $\left(\mathrm{T}_{\mathrm{c}}\right)$ and relative humidity $\left(\mathrm{H}_{\mathrm{R}}\right)$ of the chamber is displayed on the left hand side of the control panel (Figure A.1c). The temperature and fill-level of the $\mathrm{LN}_{2}$ bath is also shown. Typical values when plunging $\mathrm{Si}_{3} \mathrm{~N}_{4}$ windows are: $\mathrm{T}_{\mathrm{c}}=20^{\circ} \mathrm{C}$ and $\mathrm{H}_{\mathrm{R}}=99 \%$. The temperature of the $\mathrm{LN}_{2}$ bath is cooled to $-196^{\circ} \mathrm{C}$.
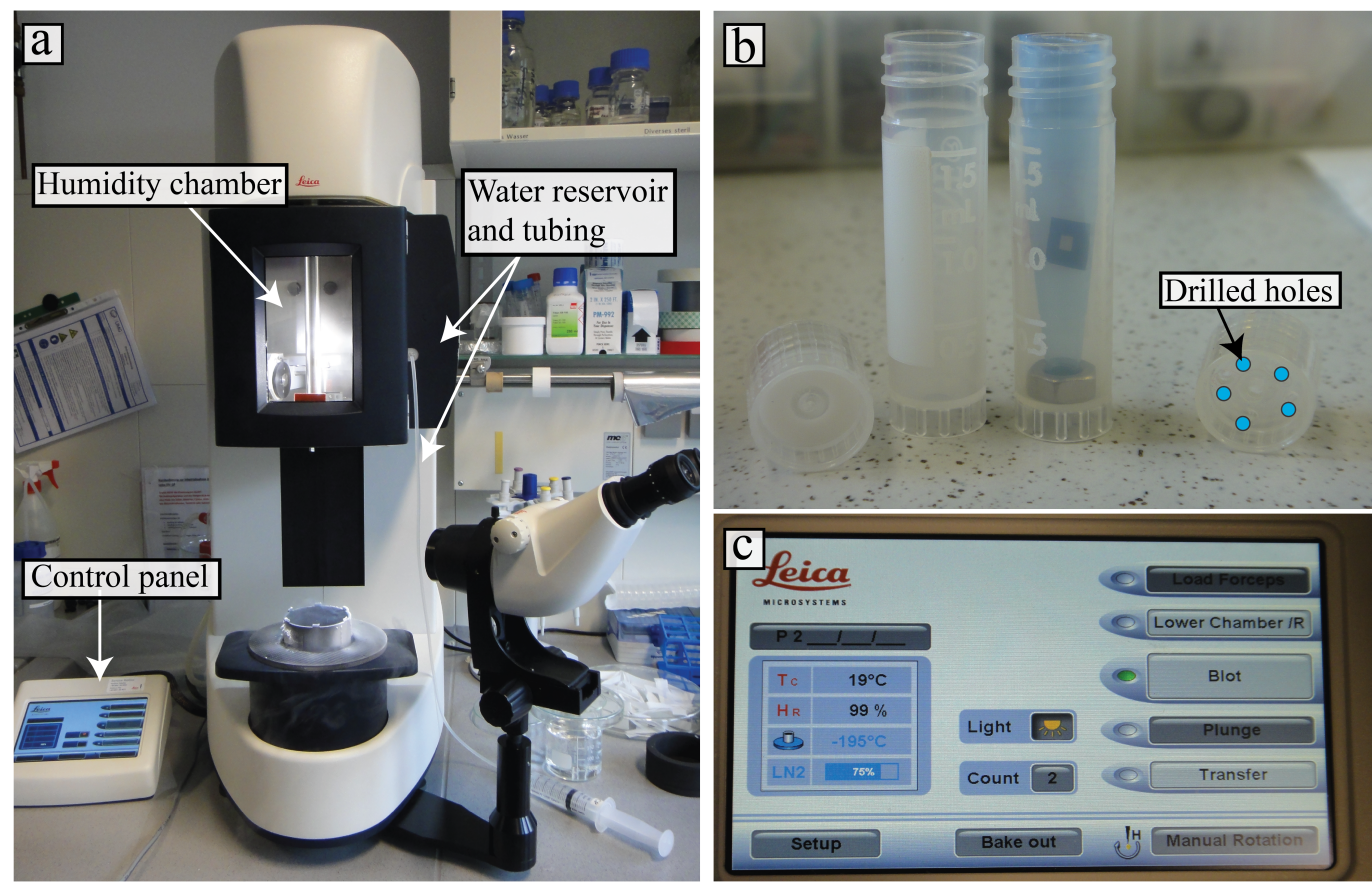

Figure A.1: a) Overview of the Leica EM GP grid plunger. b) Difference between an unprepared and prepared cryovial. The drilled holes are highlighted in blue. c) Zoom-in of the control panel. The settings are shown on the left side and the current configuration of the grid plunger is shown on the right side.

Once the $\mathrm{LN}_{2}$ bath reaches $-196{ }^{\circ} \mathrm{C}$, the gaseous ethane/propane tank is connected to the ethane/propane reservoir (Figure A.2a) via the gaseous adapter (Figure A.2b,c). The cool temperature of the surrounding $\mathrm{LN}_{2}$ reservoir allows the ethane/propane to condense into a liquid. The water reservoir has a capacity of $60 \mathrm{~mL}$ and is filled up using ultra-pure water. Once the water reservoir is filled, the chamber's humidifier is activated, as indicated by the steam shown throughout Figure A.2e.

The grid plunging process begins by gently rinsing a $\mathrm{Si}_{3} \mathrm{~N}_{4}$ window with ultra-pure water. To ensure the cells do not begin to dry out, a droplet of water is placed onto both sides of the window after it has been rinsed. A pair of tweezers is used to hold the $\mathrm{Si}_{3} \mathrm{~N}_{4}$ window throughout the grid plunging process (Figure A.2d). The various 
plunging steps are listed on the right hand side of the control panel. The tweezers are attached to the plunger in the Load Forceps position. Once the forceps have been loaded, Lower Chamber brings the tweezers into the humidity chamber. The Blot process is subsequently executed: The Leica EM GP grid plunger is designed to blot samples automatically. However, due to the small size of the $\mathrm{Si}_{3} \mathrm{~N}_{4}$ window, the blotting process must be done manually (Figure A.2e). Assuming the cells are adhered to the flat side of the $\mathrm{Si}_{3} \mathrm{~N}_{4}$ window, the window frame of the concave side is blotted first. This is done to maximize the time the cells are in a liquid environment. After both the concave and flat side of the window frame have been blotted, the sample is Plunged into the liquid ethane/propane bath, as shown in Figure A.2f. The sample is then transferred into the prepared cryovial (Figure A.2f) and placed into the revolver (Figure A.2g). The revolver is submerged in $\mathrm{LN}_{2}$ until the lyophilization process starts.
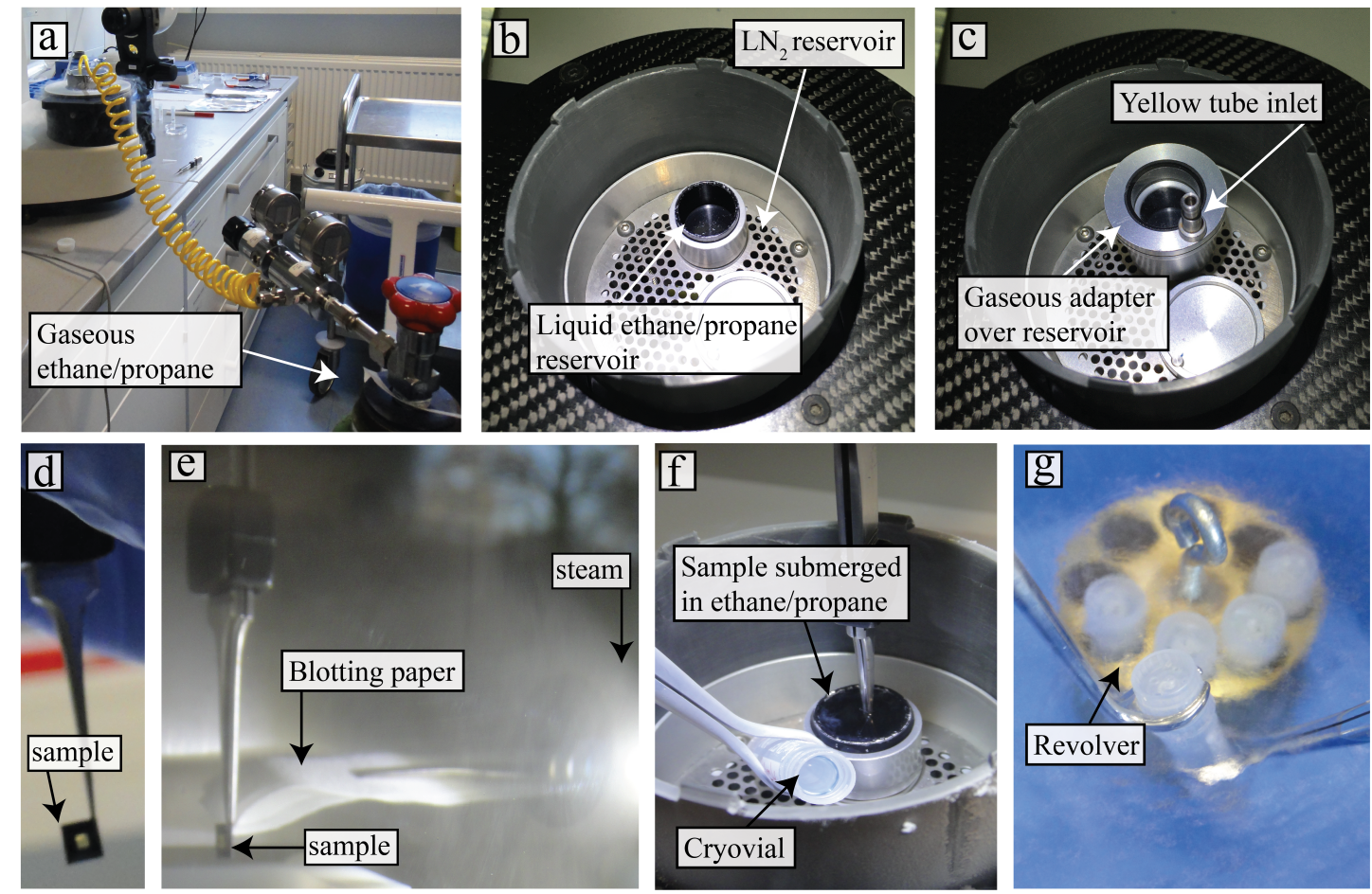

Figure A.2: a) The gaseous ethane/propane is connected to the b) reservoir using the c) gaseous adapter. The $\mathrm{Si}_{3} \mathrm{~N}_{4}$ window is held by a d) pair of tweezers throughout the grid plunging process. The sample is e) manually blotted and then f) submerged into the liquid ethane/propane bath. The sample is g) transferred to the revolver and stored in liquid nitrogen.

\section{A.2 Fluorescent Labeling of DNA}

The following text details the labeling process introduced in section 4.1.2. Within this thesis, the Thermo Fisher Scientific Click-iT EdU Alexa Fluor 647 imaging kit (Product 
C10340) was used to fluorescently label DNA within 3T3 fibroblasts. The sequence of the staining process is as follows:

1. Incubate cultured cells with EdU (5-ethynyl-2'-deoxyuridine), a nucleoside analog of thymidine.

2. Chemically fix and permeabilize cells.

3. Label EdU with Alexa Fluor 647.

4. Acquire images.

The values listed in the following protocol were used when preparing lyophilized samples for Run 71 at P10 (November, 2017). The values and concentrations listed assume the cells are cultured in a $35 \mathrm{~mm}$ diameter Petri dish. To prepare all necessary components, see the Click-iT manual listed in reference [156].

1. Split $400 \mu \mathrm{L}$ of cell suspension into a $35 \mathrm{~mm}$ diameter Petri dish containing $2 \mathrm{~mL}$ medium.

2. Approximately 2 hours later, once the cells begin to adhere to the Petri dish, add $2 \mu \mathrm{L}$ of $10 \mathrm{mM}$ stock solution (EdU) and incubate $\left(37^{\circ} \mathrm{C}, 5 \% \mathrm{CO}_{2}\right)$ for $\sim 20$ hours (average doubling time).

3. Warm-up $1 \mathrm{X}$ PBS to $37^{\circ} \mathrm{C}$. Remove all medium and wash the cells 3 times with PBS for 5 minutes each.

4. Remove PBS and add $2 \mathrm{~mL}$ of $3.7 \%$ (room temperature) PFA to chemically fix the cells. Incubate at room temperature for 15 minutes.

5. Remove the PFA and wash the cells two more times with (room temperature) $3 \%$ BSA in $1 \mathrm{X}$ PBS for 5 minutes each.

6. Remove BSA-PBS and add $2 \mathrm{~mL}$ of $0.5 \%$ Triton-X100 in (room temperature) $1 \mathrm{X}$ PBS. Incubate the cells at room temperature for 20 minutes.

7. Remove Triton-PBS and wash, two times, with $3 \%$ BSA in 1X PBS for 5 minutes each. Prepare step 8 while this step is occurring.

8. Using an eppendorf tube, prepare the cocktail in the order shown below. The total volume of the cocktail is $1 \mathrm{~mL}$. The cocktail is stable for approximately 15 minutes. 
(a) To prepare the $1 \mathrm{X}$ reaction buffer, add $86 \mu \mathrm{L}$ of $10 \mathrm{X}$ Click-iT reaction buffer to $774 \mu \mathrm{L}$ of ultra-pure water. Prepare a fresh batch for each staining session; do not store.

(b) Add $40 \mu \mathrm{L}$ of $\mathrm{CuSO}_{4}$.

(c) Add $2.5 \mu \mathrm{L}$ of Alexa Fluor 647 .

(d) In a separate eppendorf tube, prepare the $1 \mathrm{X}$ buffer additive: mix $90 \mu \mathrm{L}$ ultrapure water and $10 \mu \mathrm{L}$ of $(10 \mathrm{X})$ buffer additive. Once these two components are mixed, add it to the first eppendorf tube. Prepare a fresh batch of the buffer additive for each staining session; do not store.

9. Remove the BSA-PBS and add the cocktail to the Petri dish. Incubate at room temperature for 30 minutes while the sample is on a rocker and protected from light.

10. Remove the cocktail and wash the cells one time with 3\% BSA in PBS. Protect from light.

11. Remove the BSA-PBS and replace with PBS. Store at $4{ }^{\circ} \mathrm{C}$ and protect from light.

An example of a labeled nucleus in the lyophilized and chemically fixed-hydrated state using this protocol is shown in Figure 6.2. 



\section{Supplementary Data of Lyophilized Samples}

In this section, data belonging to 33 lyophilized NIH-3T3 fibroblasts is shown. The data is supplementary to the work presented in chapter 5. Section 5.2.2 qualitatively describes how the subsequent data is produced.

For each of the 33 cells, the (a) dark field image is calculated using the entire usable data range $\left[q_{0}, q_{\max }\right]=[0.038,0.867] \mathrm{nm}^{-1}$, corresponding to length scales in the $[7,165]$ $\mathrm{nm}$ range in real space. The (b) manually-selected regions of interest are shown: The heterochromatin and euchromatin are shown in orange and blue, respectively, and the cytoplasm and background are shown in gray and black, respectively. The (c) average diffraction pattern belonging to each region of interest is azimuthally integrated, background-corrected and plotted against the absolute value of the scattering vector $q$. The bold dashed lines are fits of $I(q)=K q^{\alpha}+B$. The solid black vertical lines at $q=$ $0.038 \mathrm{~nm}^{-1}$ and $q=0.867 \mathrm{~nm}^{-1}$ represent $q_{0}$ and $q_{\max }$, respectively. The dashed vertical lines represent $q_{\min }$ of the respective region of interest. The solid green lines are proportional to $q^{-4}$ and serve only as a visual aid to the overall $I(q)$ decay. (d) Map of the exponent $\alpha$, derived by fitting individual $I(q)$ profiles to $I(q)=K q^{\alpha}+B$ in the $\left[q_{0}, q_{\text {min }}\right]$ range. (e) Map of the Porod constant $K$, derived by fitting individual $I(q)$ profiles to $I(q)=K q^{\alpha}+B$ in the $\left[q_{\min }, q_{\max }\right]$ range. The (f) map of the reconstructed phase, rendered using the single distance RAAR [105] algorithm with 500 iteration rounds. The projected mass density and projected electron density are also shown. Furthermore, the mean value of each variable, per region of interest, is listed. 
a)
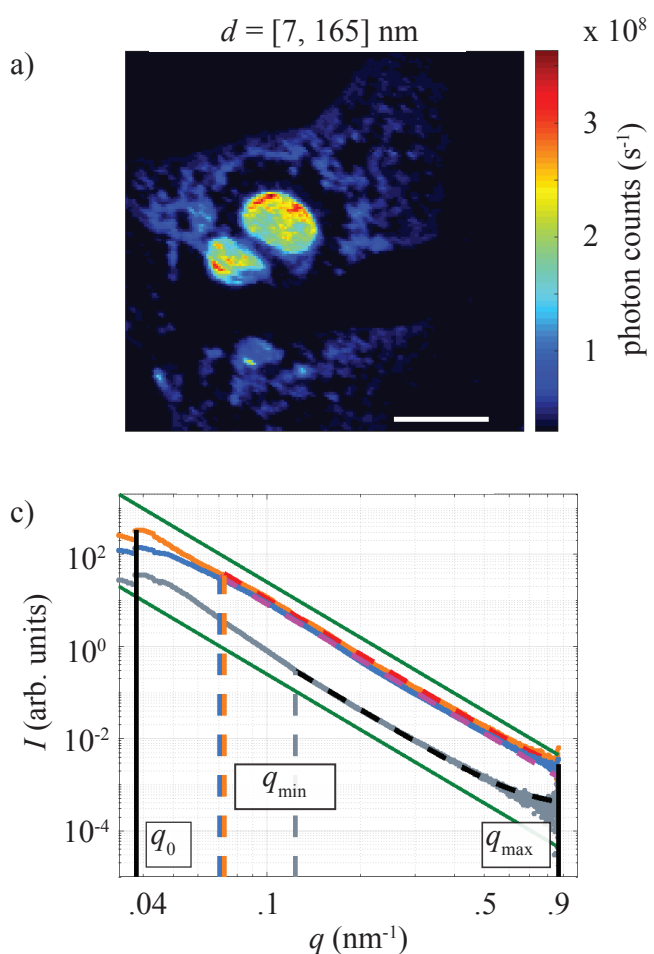

b)

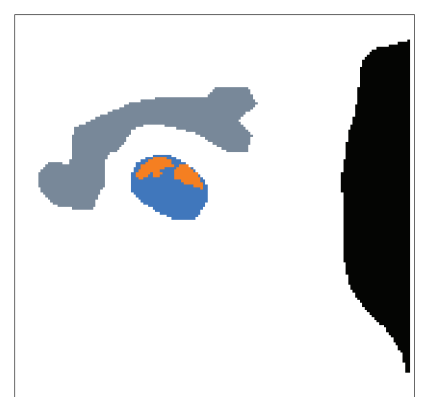

d)

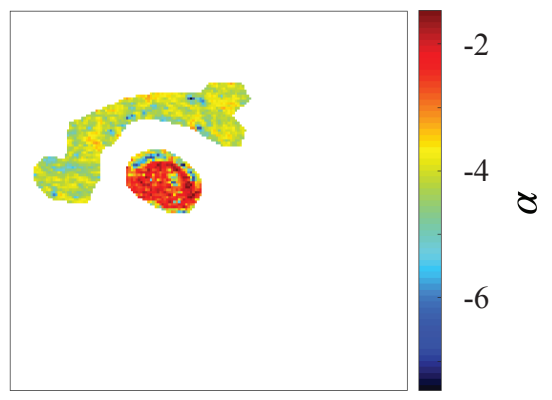

e)

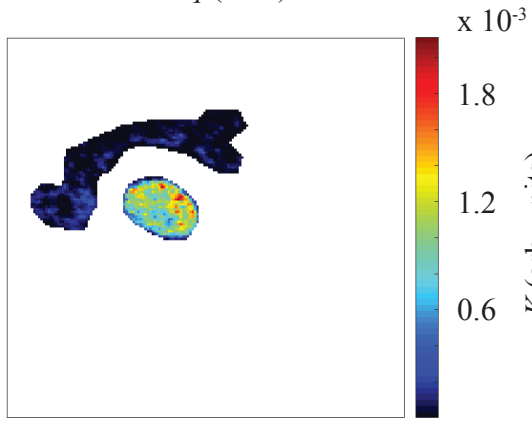

$10^{-3}$

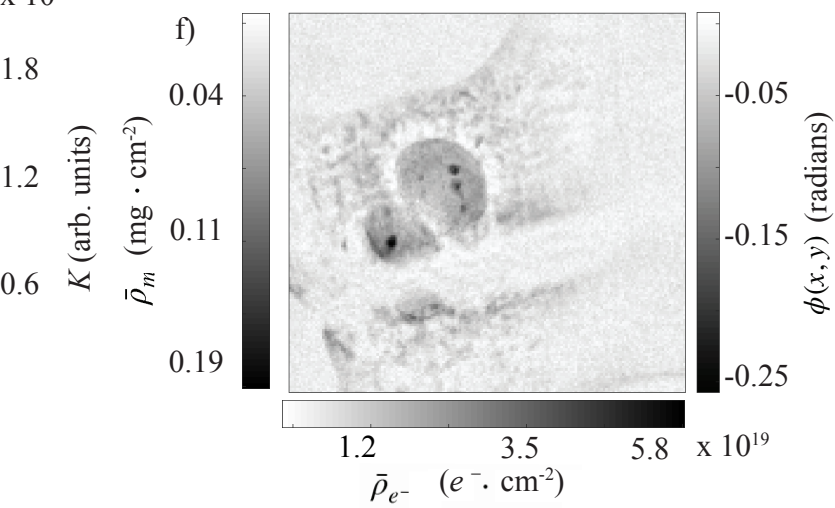

Figure B.1: Sample AW-PD06-C4. The scale bar in a) is $10 \mu \mathrm{m}$ and applies to subfigures b), d), e) and f). See page 117.

\begin{tabular}{|c|c|c|c|c|c|c|}
\hline Sample & ROI & $\alpha$ & $K$ (arb.units) & $-\phi(\mathrm{rad})$ & $\bar{\rho}_{m}\left(\frac{\mathrm{mg}}{\mathrm{cm}^{2}}\right)$ & $\bar{\rho}_{e^{-}}\left(\frac{\mathrm{e}^{-}}{\mathrm{cm}^{2}}\right)$ \\
\hline \multirow{3}{*}{ AW-PD06-C4 } & Heterochromatin & -3.90 & $1.09 \times 10^{-3}$ & 0.09 & 0.07 & $2.13 \times 10^{19}$ \\
& Euchromatin & -2.80 & $8.35 \times 10^{-4}$ & 0.08 & 0.06 & $1.74 \times 10^{19}$ \\
& Cytoplasm & -4.18 & $5.45 \times 10^{-5}$ & 0.04 & 0.03 & $8.33 \times 10^{18}$ \\
\hline
\end{tabular}


a)

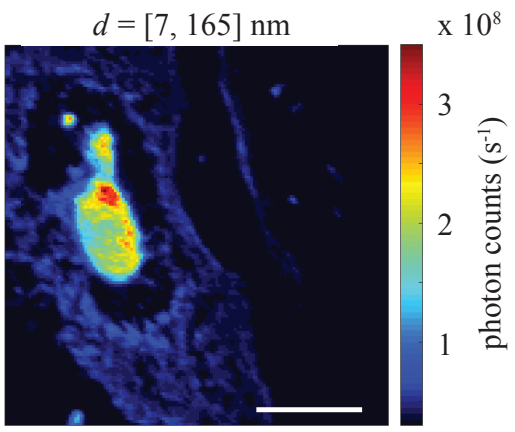

c)

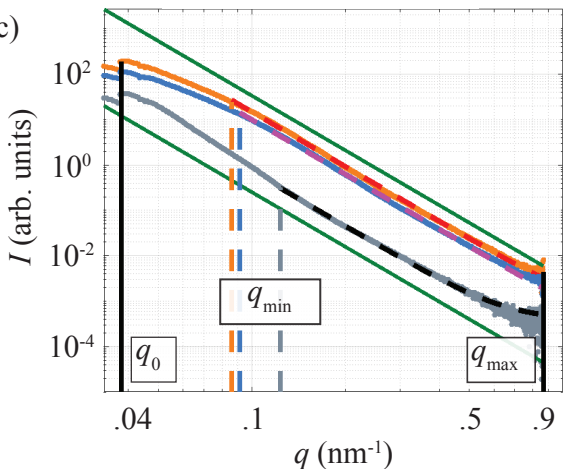

b)

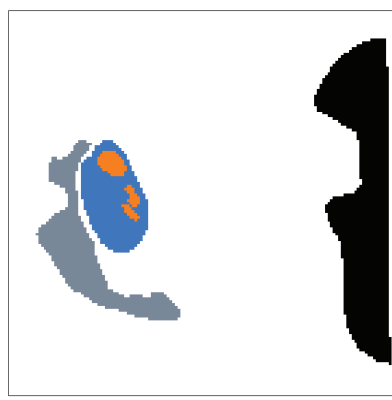

d)
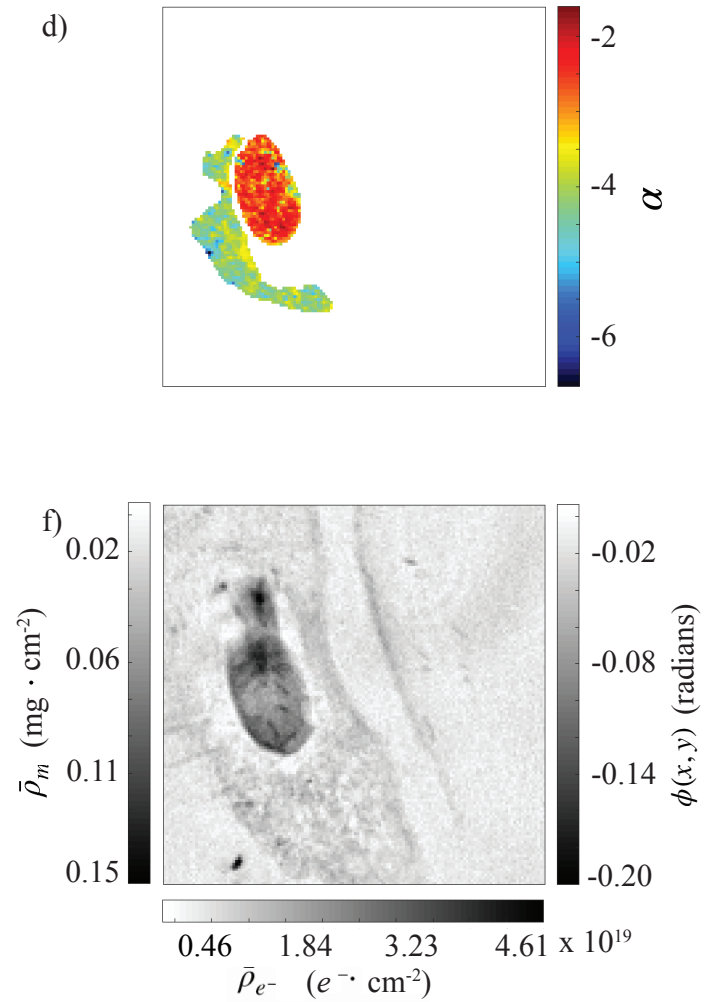

Figure B.2: Sample AW-PD06-C5. The scale bar in a) is $10 \mu \mathrm{m}$ and applies to subfigures b), d), e) and f). See page 117 .

\begin{tabular}{|c|c|c|c|c|c|c|}
\hline Sample & ROI & $\alpha$ & $K$ (arb.units) & $-\phi(\mathrm{rad})$ & $\bar{\rho}_{m}\left(\frac{\mathrm{mg}}{\mathrm{cm}^{2}}\right)$ & $\bar{\rho}_{e^{-}}\left(\frac{\mathrm{e}^{-}}{\mathrm{cm}^{2}}\right)$ \\
\hline \multirow{3}{*}{ AW-PD06-C5 } & Heterochromatin & -2.80 & $1.48 \times 10^{-3}$ & 0.12 & 0.09 & $2.75 \times 10^{19}$ \\
& Euchromatin & -2.69 & $9.84 \times 10^{-4}$ & 0.10 & 0.07 & $2.24 \times 10^{19}$ \\
& Cytoplasm & -4.17 & $5.38 \times 10^{-5}$ & 0.03 & 0.02 & $5.84 \times 10^{18}$ \\
\hline
\end{tabular}


a)

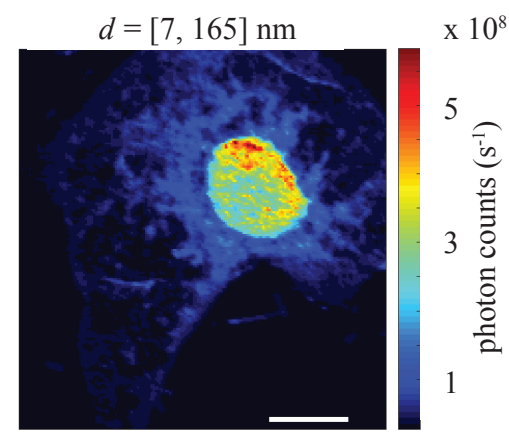

c)

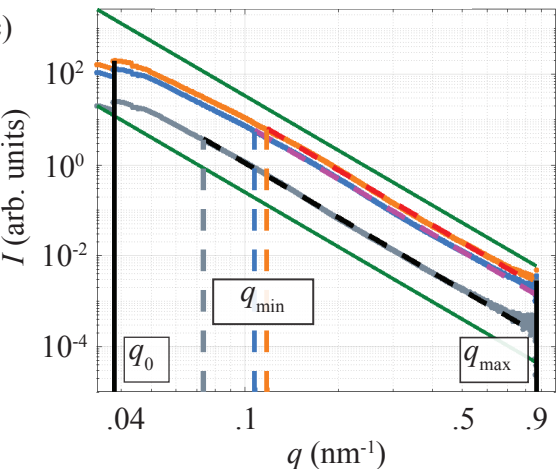

b)

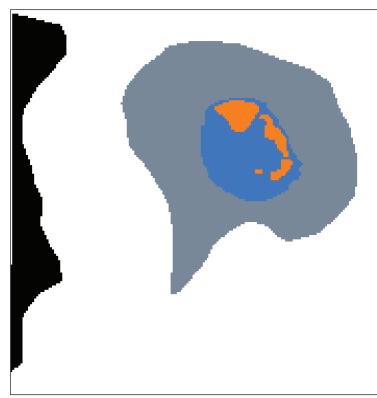

d)
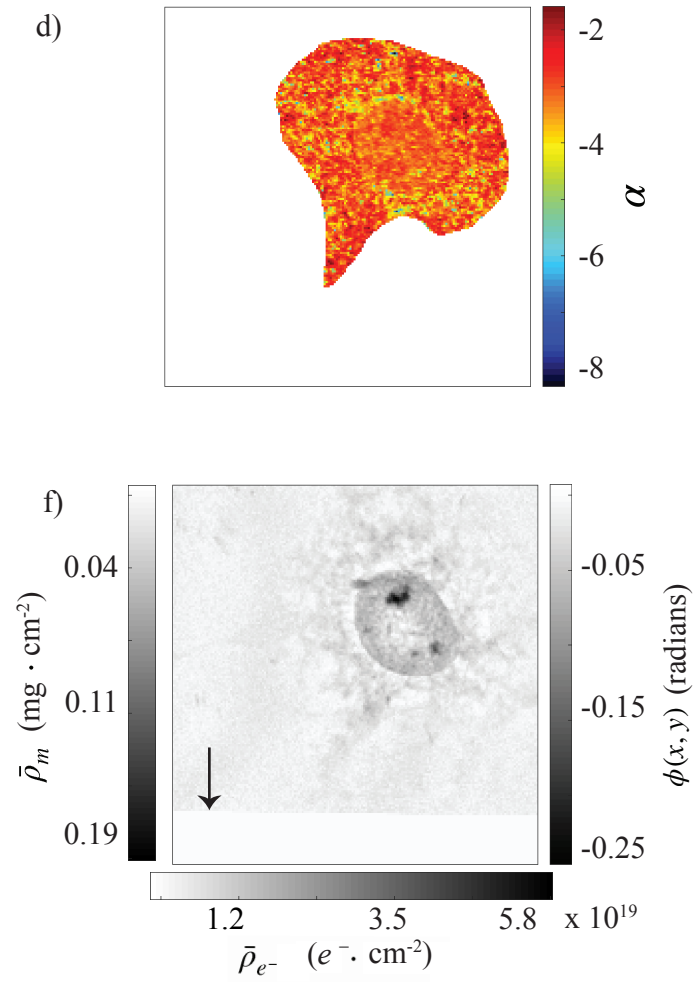

Figure B.3: Sample AW-PD06-C6. The scale bar in a) is $10 \mu \mathrm{m}$ and applies to subfigures $\mathrm{b}$ ), d), e) and $\mathrm{f}$ ). In $\mathrm{f}$ ), the black arrow indicates a region where X-ray holography data was not collected. See page 117.

\begin{tabular}{|c|c|c|c|c|c|c|}
\hline Sample & ROI & $\alpha$ & $K$ (arb.units) & $-\phi(\mathrm{rad})$ & $\bar{\rho}_{m}\left(\frac{\mathrm{mg}}{\mathrm{cm}^{2}}\right)$ & $\bar{\rho}_{e^{-}}\left(\frac{\mathrm{e}^{-}}{\mathrm{cm}^{2}}\right)$ \\
\hline \multirow{3}{*}{ AW-PD06-C6 } & Heterochromatin & -3.24 & $1.22 \times 10^{-3}$ & 0.09 & 0.07 & $1.99 \times 10^{19}$ \\
& Euchromatin & -3.23 & $7.73 \times 10^{-4}$ & 0.05 & 0.04 & $1.14 \times 10^{19}$ \\
& Cytoplasm & -3.13 & $1.09 \times 10^{-4}$ & 0.02 & 0.02 & $4.74 \times 10^{18}$ \\
\hline
\end{tabular}


a)

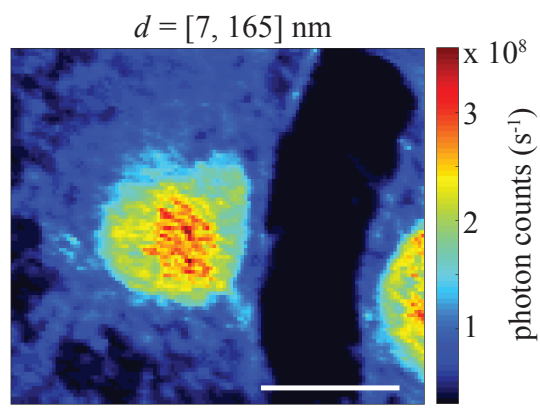

c)

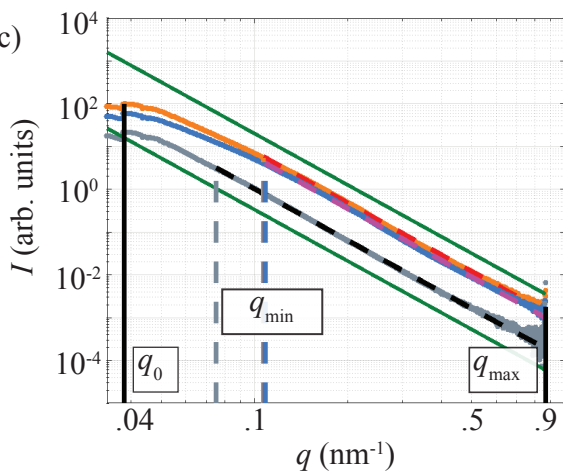

b)

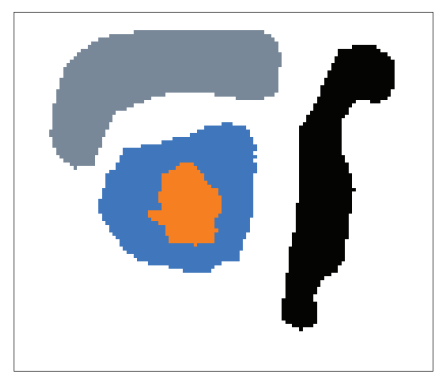

d)

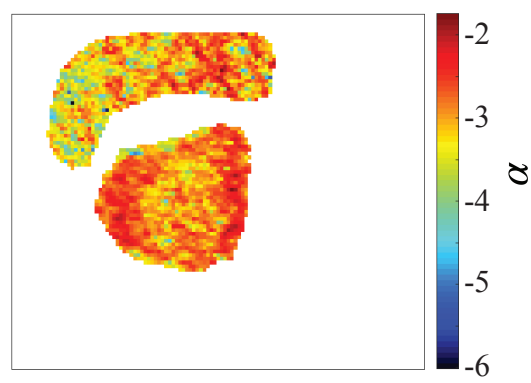

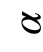

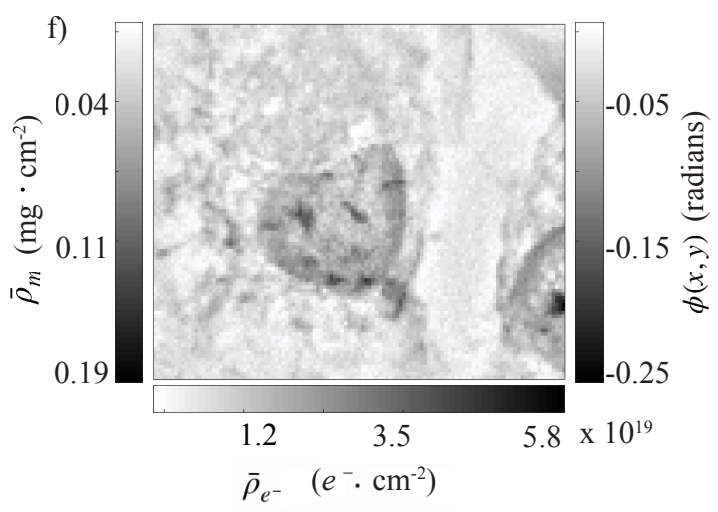

Figure B.4: Sample AW-PD06-C7A. The scale bar in a) is $10 \mu \mathrm{m}$ and applies to subfigures b), d), e) and f). See page 117 .

\begin{tabular}{|c|c|c|c|c|c|c|}
\hline Sample & ROI & $\alpha$ & $K$ (arb.units) & $-\phi(\mathrm{rad})$ & $\bar{\rho}_{m}\left(\frac{\mathrm{mg}}{\mathrm{cm}^{2}}\right)$ & $\bar{\rho}_{e^{-}}\left(\frac{\mathrm{e}^{-}}{\mathrm{cm}^{2}}\right)$ \\
\hline \multirow{3}{*}{ AW-PD06-C7A } & Heterochromatin & -3.01 & $7.53 \times 10^{-4}$ & 0.07 & 0.05 & $1.56 \times 10^{19}$ \\
& Euchromatin & -2.78 & $5.64 \times 10^{-4}$ & 0.06 & 0.04 & $1.30 \times 10^{19}$ \\
& Cytoplasm & -3.15 & $9.84 \times 10^{-5}$ & 0.03 & 0.02 & $6.87 \times 10^{18}$ \\
\hline
\end{tabular}


a)

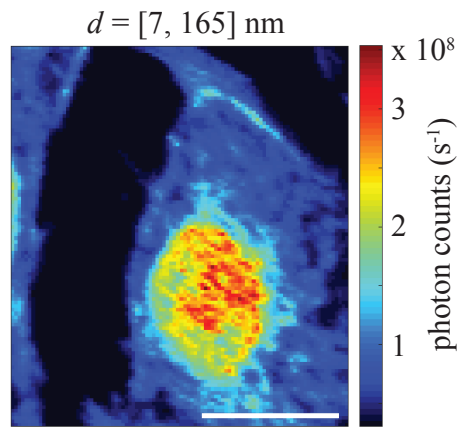

c)

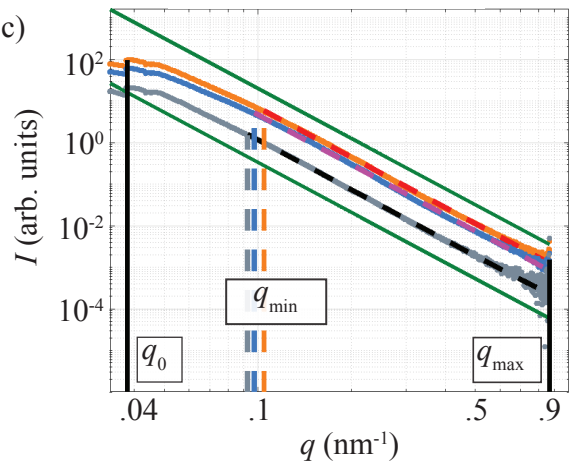

e)

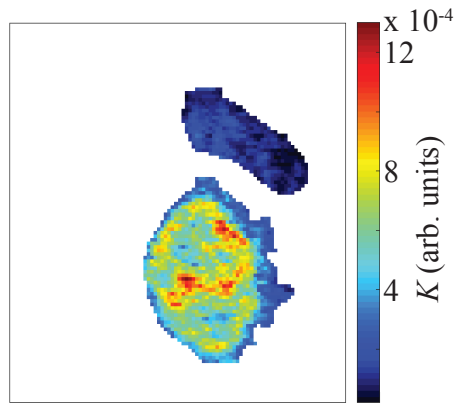

b)

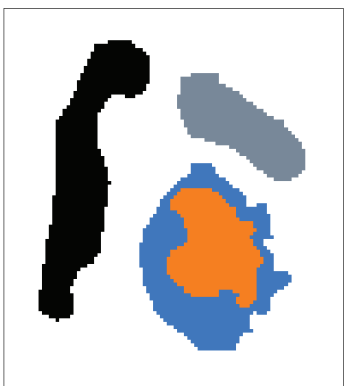

d)
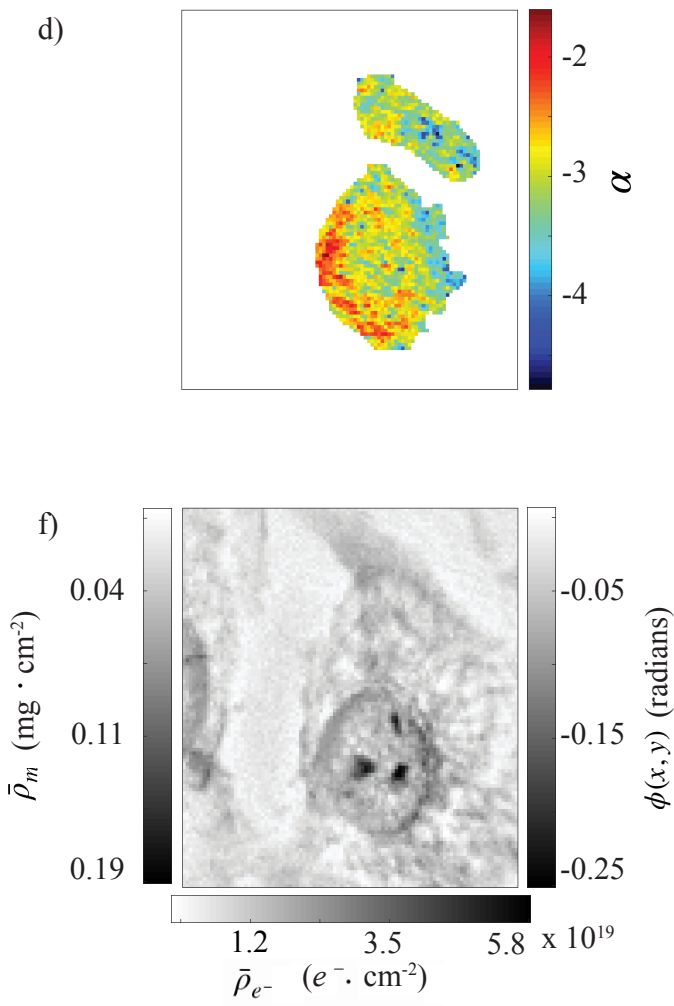

Figure B.5: Sample AW-PD06-C7B. The scale bar in a) is $10 \mu \mathrm{m}$ and applies to subfigures b), d), e) and f). See page 117 .

\begin{tabular}{|c|c|c|c|c|c|c|}
\hline Sample & ROI & $\alpha$ & $K$ (arb.units) & $-\phi(\mathrm{rad})$ & $\bar{\rho}_{m}\left(\frac{\mathrm{mg}}{\mathrm{cm}^{2}}\right)$ & $\bar{\rho}_{e^{-}}\left(\frac{\mathrm{e}^{-}}{\mathrm{cm}^{2}}\right)$ \\
\hline \multirow{3}{*}{ AW-PD06-C7B } & Heterochromatin & -3.01 & $7.04 \times 10^{-4}$ & 0.09 & 0.07 & $2.10 \times 10^{19}$ \\
& Euchromatin & -2.89 & $4.70 \times 10^{-4}$ & 0.07 & 0.05 & $1.54 \times 10^{19}$ \\
& Cytoplasm & -3.21 & $1.11 \times 10^{-4}$ & 0.04 & 0.03 & $1.03 \times 10^{19}$ \\
\hline
\end{tabular}


a)

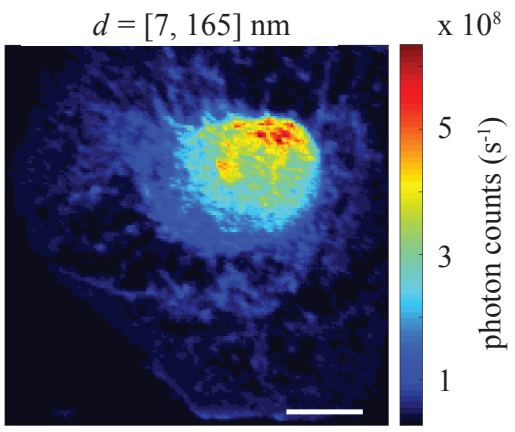

c)

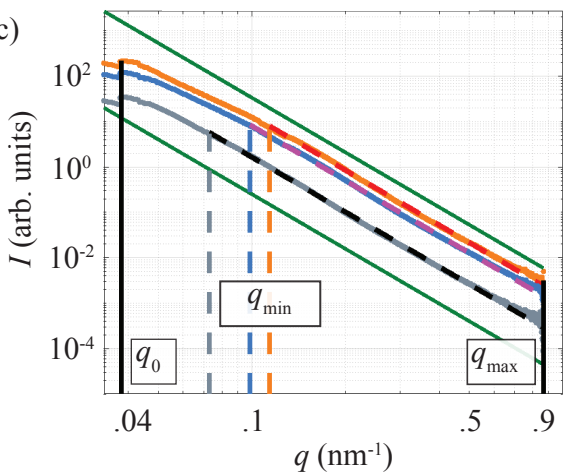

b)

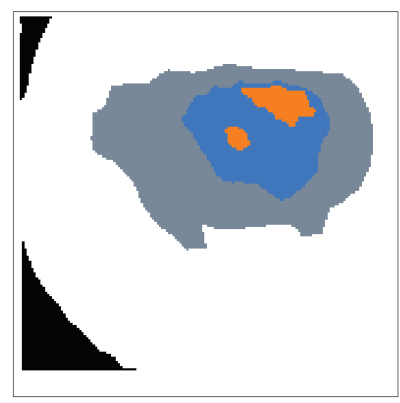

d)

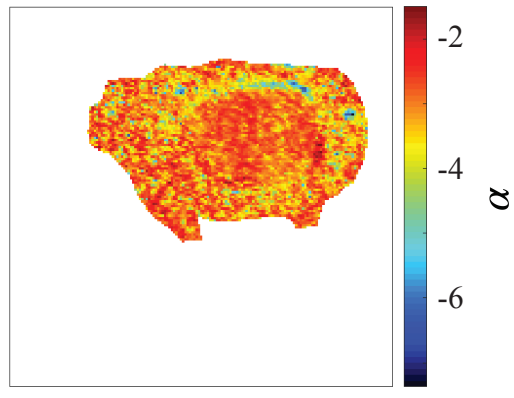

e)

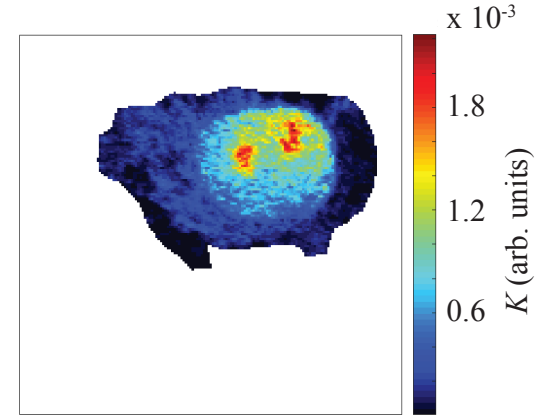

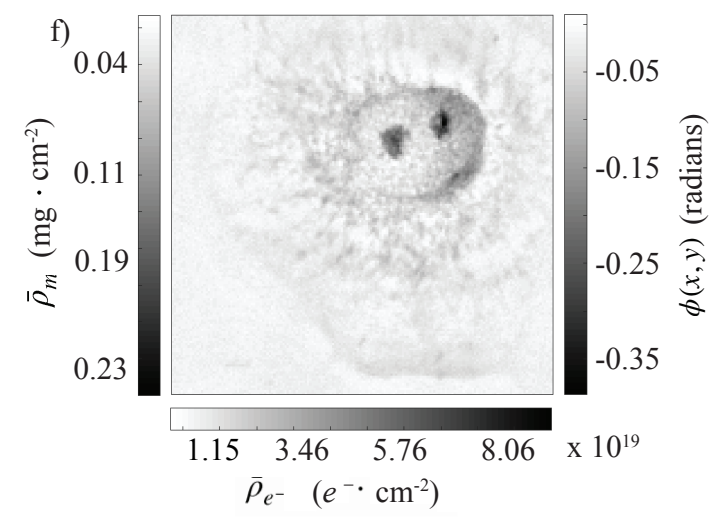

Figure B.6: Sample AW-PD06-C8. The scale bar in a) is $10 \mu \mathrm{m}$ and applies to subfigures b), d), e) and f). See page 117 .

\begin{tabular}{|c|c|c|c|c|c|c|}
\hline Sample & ROI & $\alpha$ & $K$ (arb.units) & $-\phi(\mathrm{rad})$ & $\bar{\rho}_{m}\left(\frac{\mathrm{mg}}{\mathrm{cm}^{2}}\right)$ & $\bar{\rho}_{e^{-}}\left(\frac{\mathrm{e}^{-}}{\mathrm{cm}^{2}}\right)$ \\
\hline \multirow{3}{*}{ AW-PD06-C8 } & Heterochromatin & -3.26 & $1.35 \times 10^{-3}$ & 0.15 & 0.11 & $3.40 \times 10^{19}$ \\
& Euchromatin & -3.04 & $7.97 \times 10^{-4}$ & 0.07 & 0.06 & $1.70 \times 10^{19}$ \\
& Cytoplasm & -3.12 & $1.62 \times 10^{-4}$ & 0.04 & 0.03 & $8.25 \times 10^{18}$ \\
\hline
\end{tabular}


a)

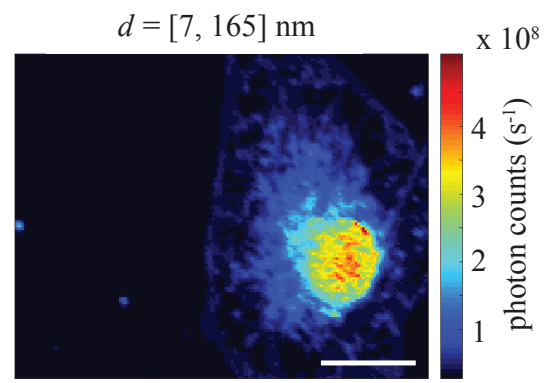

c)

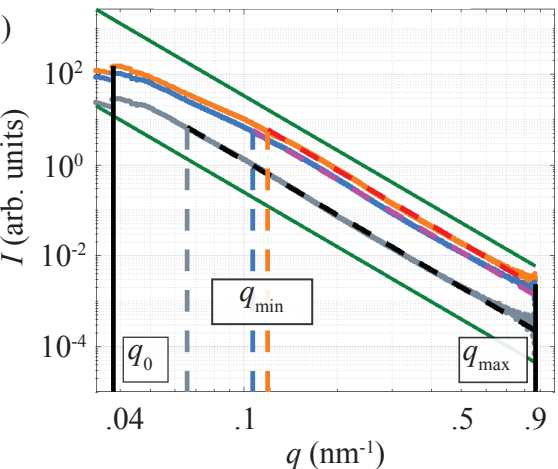

b)

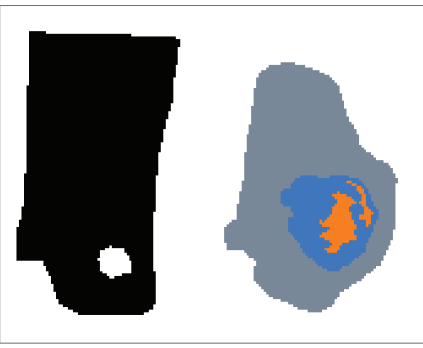

d)

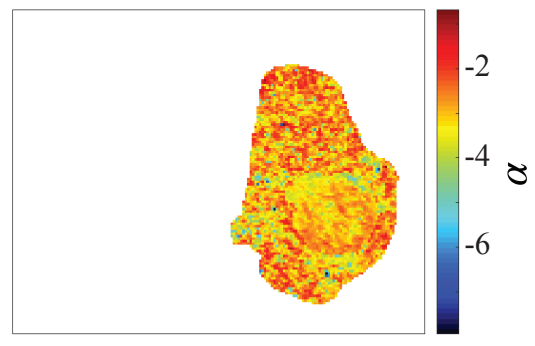

x $10^{-3}$

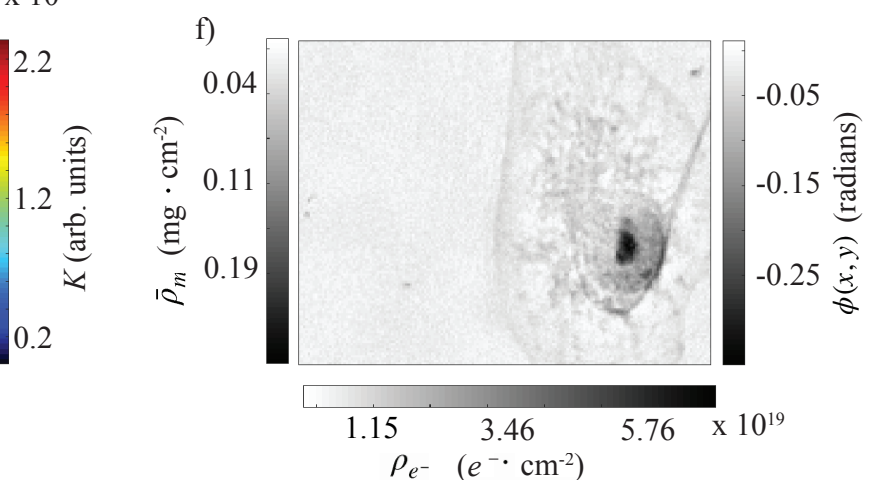

Figure B.7: Sample AW-PD06-C10. The scale bar in a) is $10 \mu \mathrm{m}$ and applies to subfigures b), d), e) and f). See page 117 .

\begin{tabular}{|c|c|c|c|c|c|c|}
\hline Sample & ROI & $\alpha$ & $K$ (arb.units) & $-\phi(\mathrm{rad})$ & $\bar{\rho}_{m}\left(\frac{\mathrm{mg}}{\mathrm{cm}^{2}}\right)$ & $\bar{\rho}_{e^{-}}\left(\frac{\mathrm{e}^{-}}{\mathrm{cm}^{2}}\right)$ \\
\hline \multirow{3}{*}{ AW-PD06-C10 } & Heterochromatin & -3.05 & $1.21 \times 10^{-3}$ & 0.18 & 0.14 & $4.09 \times 10^{19}$ \\
& Euchromatin & -3.12 & $7.48 \times 10^{-4}$ & 0.08 & 0.06 & $1.93 \times 10^{19}$ \\
& Cytoplasm & -2.89 & $1.27 \times 10^{-4}$ & 0.04 & 0.03 & $8.55 \times 10^{18}$ \\
\hline
\end{tabular}


a)

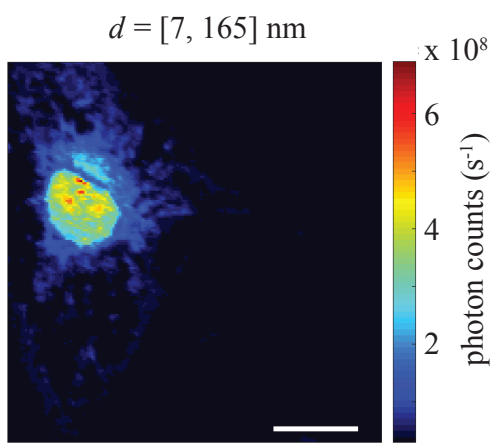

c)

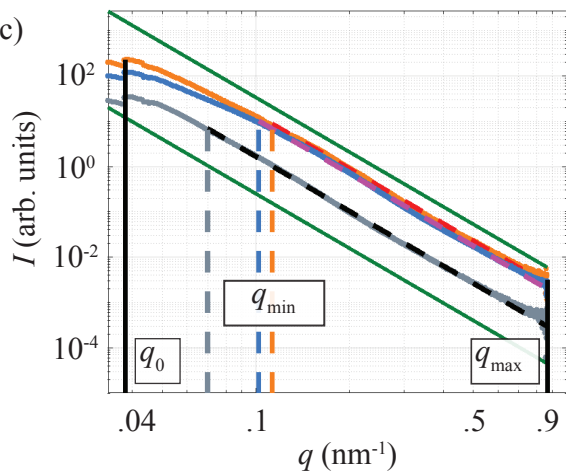

b)

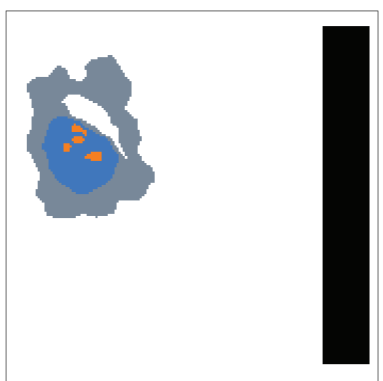

d)

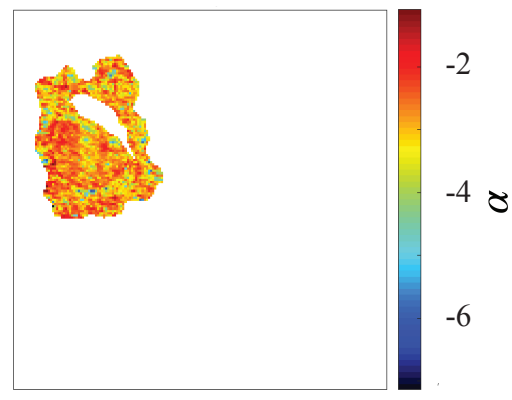

e)

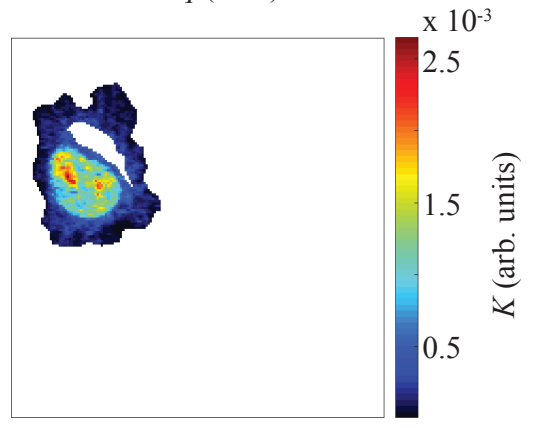

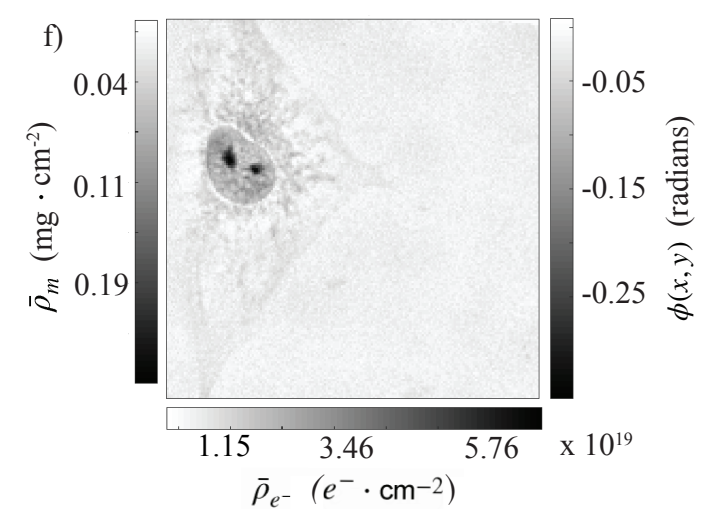

Figure B.8: Sample AW-PD06-C11. The scale bar in a) is $10 \mu \mathrm{m}$ and applies to subfigures b), d), e) and f). See page 117 .

\begin{tabular}{|c|c|c|c|c|c|c|}
\hline Sample & ROI & $\alpha$ & $K$ (arb.units) & $-\phi(\mathrm{rad})$ & $\bar{\rho}_{m}\left(\frac{\mathrm{mg}}{\mathrm{cm}^{2}}\right)$ & $\bar{\rho}_{e^{-}}\left(\frac{\mathrm{e}^{-}}{\mathrm{cm}^{2}}\right)$ \\
\hline \multirow{3}{*}{ AW-PD06-C11 } & Heterochromatin & -3.17 & $1.40 \times 10^{-3}$ & 0.15 & 0.12 & $3.54 \times 10^{19}$ \\
& Euchromatin & -2.80 & $1.12 \times 10^{-3}$ & 0.10 & 0.08 & $2.35 \times 10^{19}$ \\
& Cytoplasm & -2.94 & $1.66 \times 10^{-4}$ & 0.03 & 0.02 & $7.42 \times 10^{18}$ \\
\hline
\end{tabular}


a)

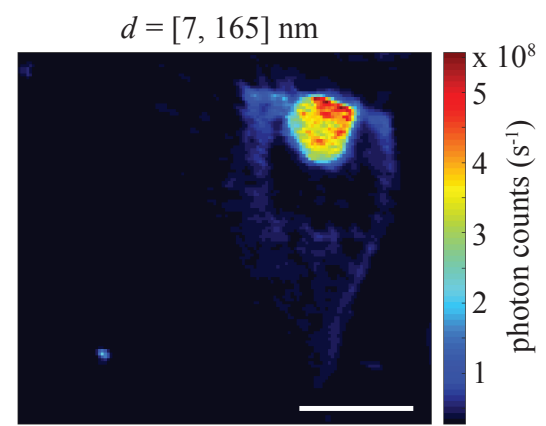

c)

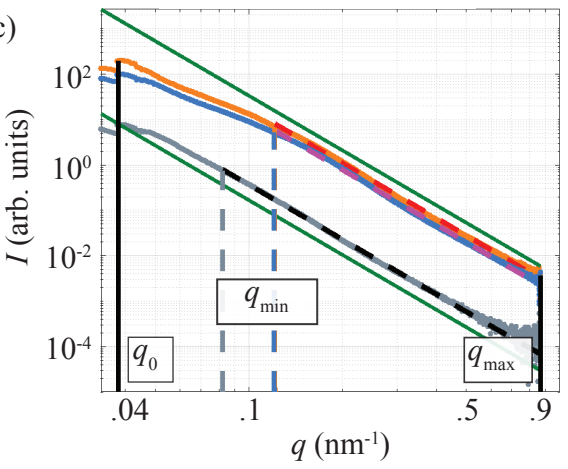

b)

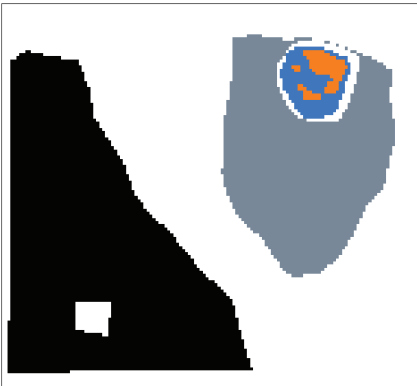

d)
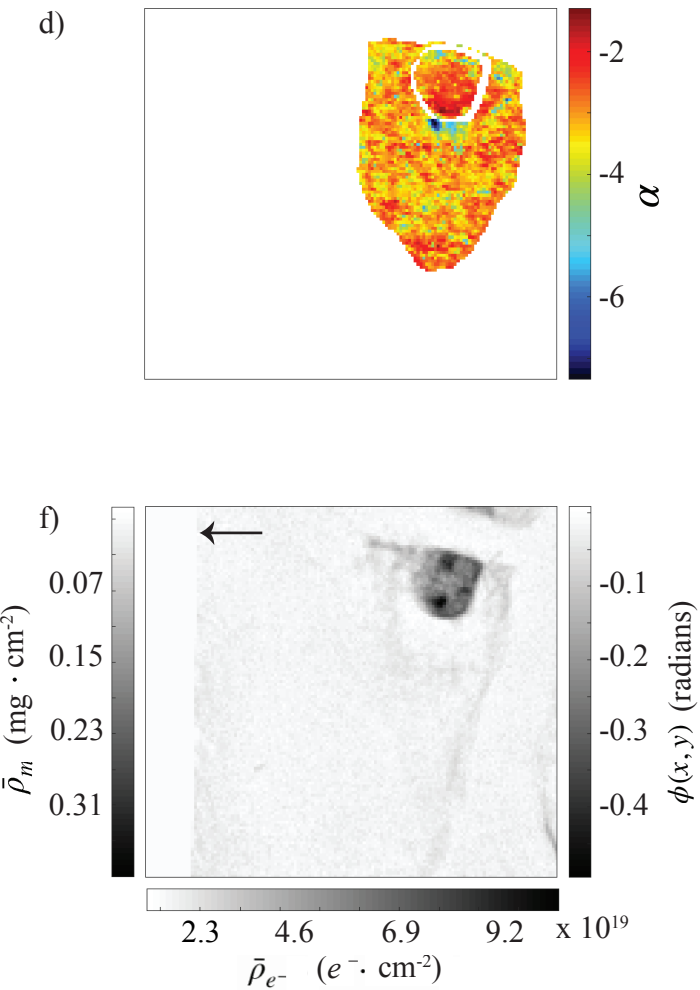

Figure B.9: Sample AW-PD06-C12. The scale bar in a) is $10 \mu \mathrm{m}$ and applies to subfigures b), d), e) and $\mathrm{f}$ ). In $\mathrm{f}$ ), the black arrow indicates a region where X-ray holography data was not collected. See page 117 .

\begin{tabular}{|c|c|c|c|c|c|c|}
\hline Sample & ROI & $\alpha$ & $K$ (arb.units) & $-\phi(\mathrm{rad})$ & $\bar{\rho}_{m}\left(\frac{\mathrm{mg}}{\mathrm{cm}^{2}}\right)$ & $\bar{\rho}_{e^{-}}\left(\frac{\mathrm{e}^{-}}{\mathrm{cm}^{2}}\right)$ \\
\hline \multirow{3}{*}{ AW-PD06-C12 } & Heterochromatin & -3.08 & $1.76 \times 10^{-3}$ & 0.28 & 0.22 & $6.53 \times 10^{19}$ \\
& Euchromatin & -2.79 & $1.25 \times 10^{-3}$ & 0.23 & 0.18 & $5.32 \times 10^{19}$ \\
& Cytoplasm & -3.17 & $3.46 \times 10^{-5}$ & 0.03 & 0.02 & $6.62 \times 10^{18}$ \\
\hline
\end{tabular}


a)

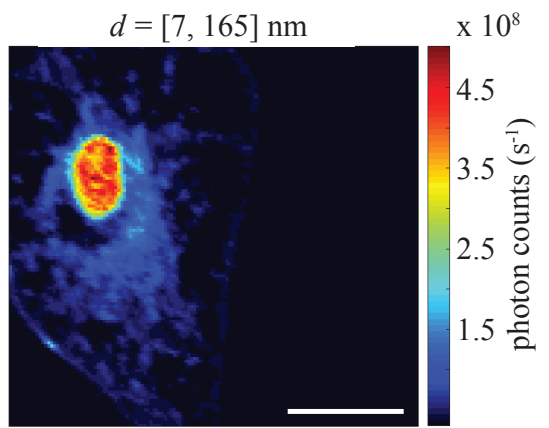

c)

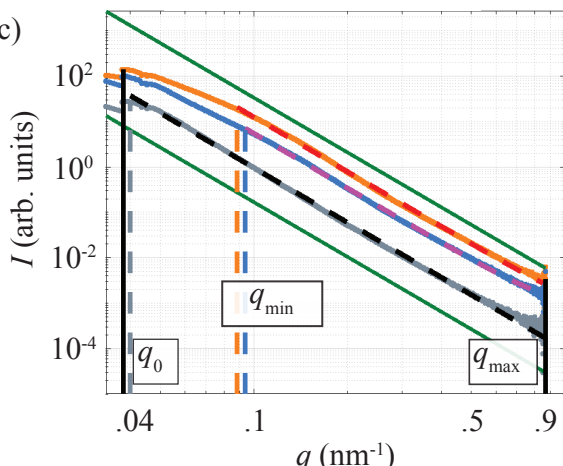

b)

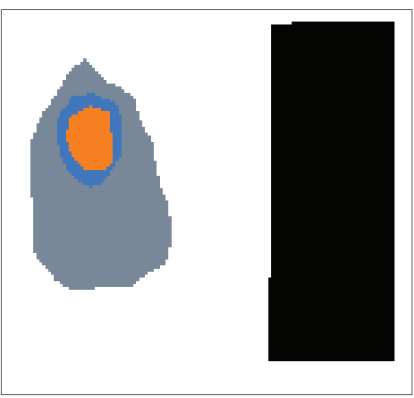

d)

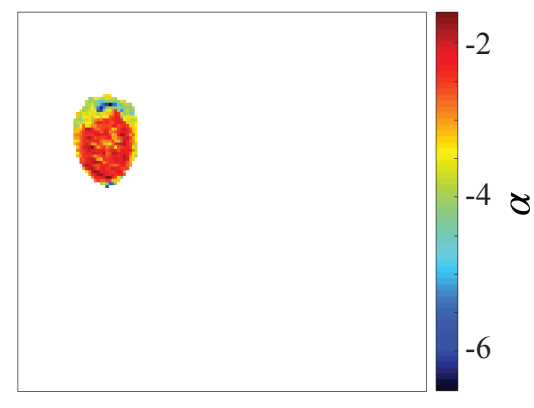

$\mathrm{x} 10^{-3}$

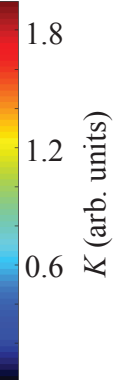

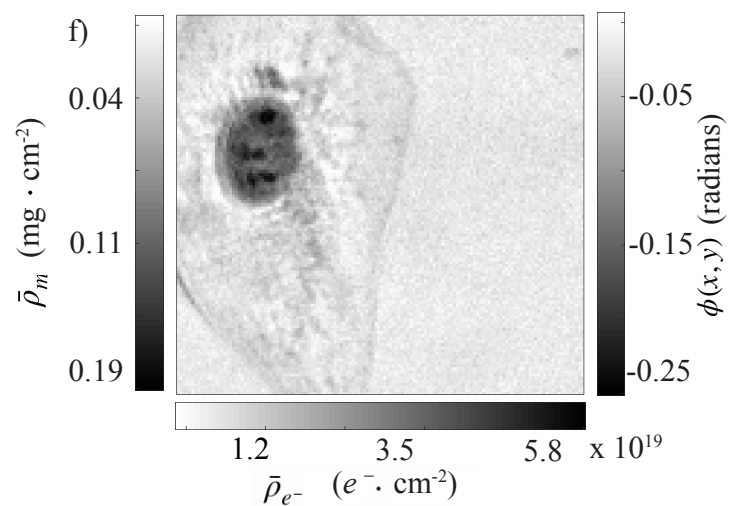

Figure B.10: Sample AW-PD06-C13. The scale bar in a) is $10 \mu \mathrm{m}$ and applies to subfigures b), d), e) and f). As shown in c), the $\left[q_{0}, q_{\min }\right]$ range for the cytoplasm (gray) is very small and only contains two data points. Consequently, no individual $I(q)$ profiles are fitted with $I(q)=K q^{\alpha}+B$ to derive the exponent. Thus, no cytoplasm data is shown in the d) exponent map. See page 117.

\begin{tabular}{|c|c|c|c|c|c|c|}
\hline Sample & ROI & $\alpha$ & $K$ (arb.units) & $-\phi(\mathrm{rad})$ & $\bar{\rho}_{m}\left(\frac{\mathrm{mg}}{\mathrm{cm}^{2}}\right)$ & $\bar{\rho}_{e^{-}}\left(\frac{\mathrm{e}^{-}}{\mathrm{cm}^{2}}\right)$ \\
\hline \multirow{3}{*}{ AW-PD06-C13 } & Heterochromatin & -2.57 & $1.26 \times 10^{-3}$ & 0.17 & 0.13 & $3.85 \times 10^{19}$ \\
& Euchromatin & -3.32 & $5.37 \times 10^{-4}$ & 0.15 & 0.12 & $3.49 \times 10^{19}$ \\
& Cytoplasm & $\mathrm{NaN}$ & $1.02 \times 10^{-4}$ & 0.05 & 0.04 & $1.09 \times 10^{19}$ \\
\hline
\end{tabular}


a)
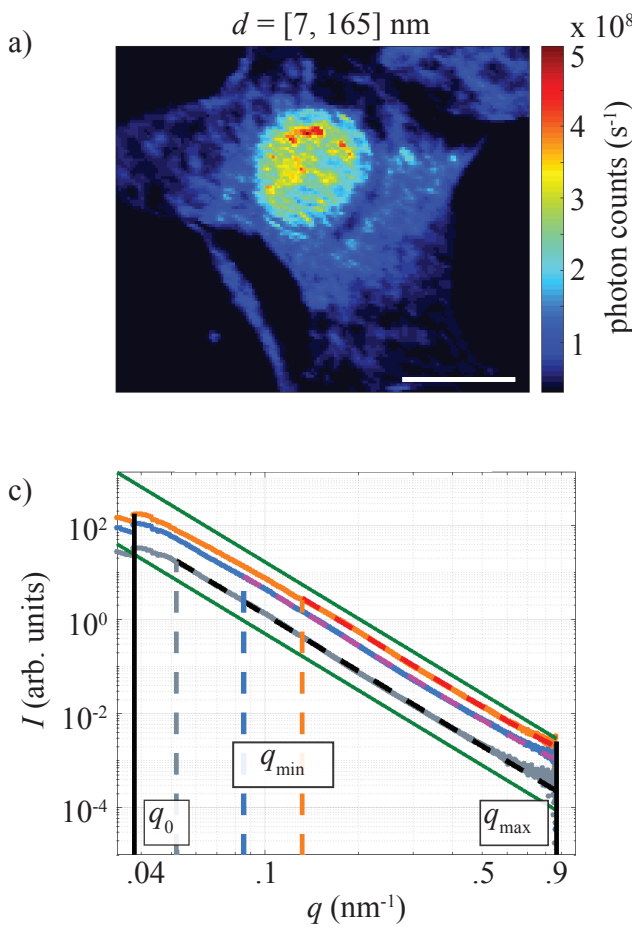

e)

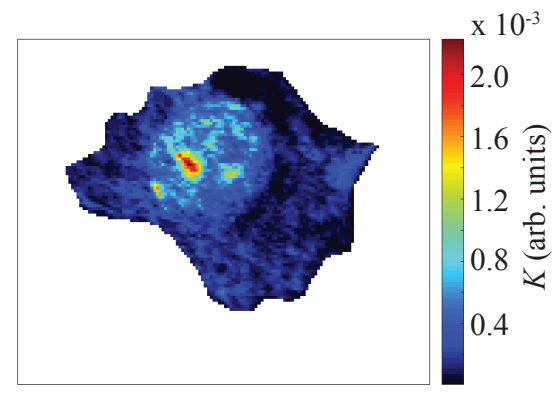

b)

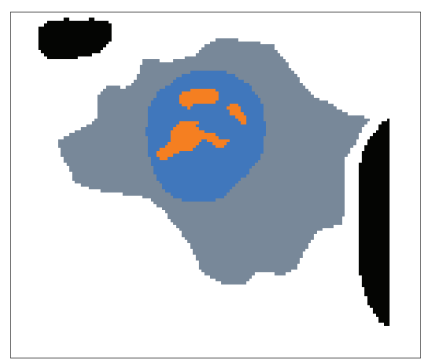

d)
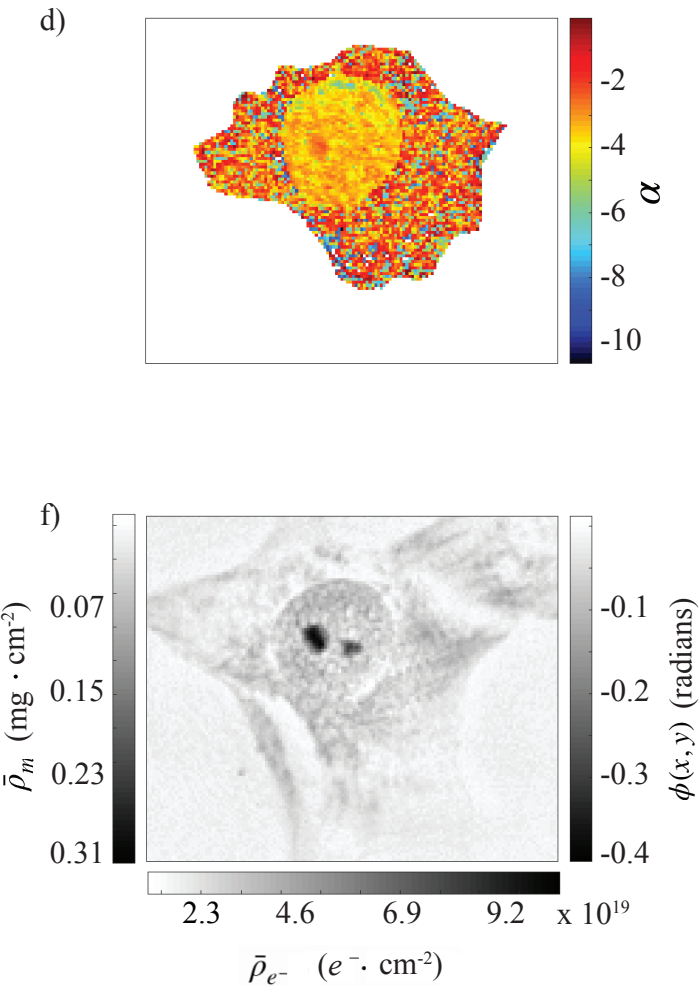

Figure B.11: Sample AW-PD06-C14. The scale bar in a) is $10 \mu \mathrm{m}$ and applies to subfigures b), d), e) and f). In the d) exponent map, the noisy data associated with the cytoplasm is attributed to the small $\left[q_{0}, q_{\text {min }}\right]$ range for this particular cell. See page 117.

\begin{tabular}{|c|c|c|c|c|c|c|}
\hline Sample & ROI & $\alpha$ & $K$ (arb.units) & $-\phi(\mathrm{rad})$ & $\bar{\rho}_{m}\left(\frac{\mathrm{mg}}{\mathrm{cm}^{2}}\right)$ & $\bar{\rho}_{e^{-}}\left(\frac{\mathrm{e}^{-}}{\mathrm{cm}^{2}}\right)$ \\
\hline \multirow{3}{*}{ AW-PD06-C14 } & Heterochromatin & -3.50 & $8.35 \times 10^{-4}$ & 0.14 & 0.11 & $3.26 \times 10^{19}$ \\
& Euchromatin & -3.51 & $4.48 \times 10^{-4}$ & 0.06 & 0.05 & $1.49 \times 10^{19}$ \\
& Cytoplasm & -3.28 & $1.37 \times 10^{-4}$ & 0.04 & 0.03 & $9.57 \times 10^{18}$ \\
\hline
\end{tabular}


a)
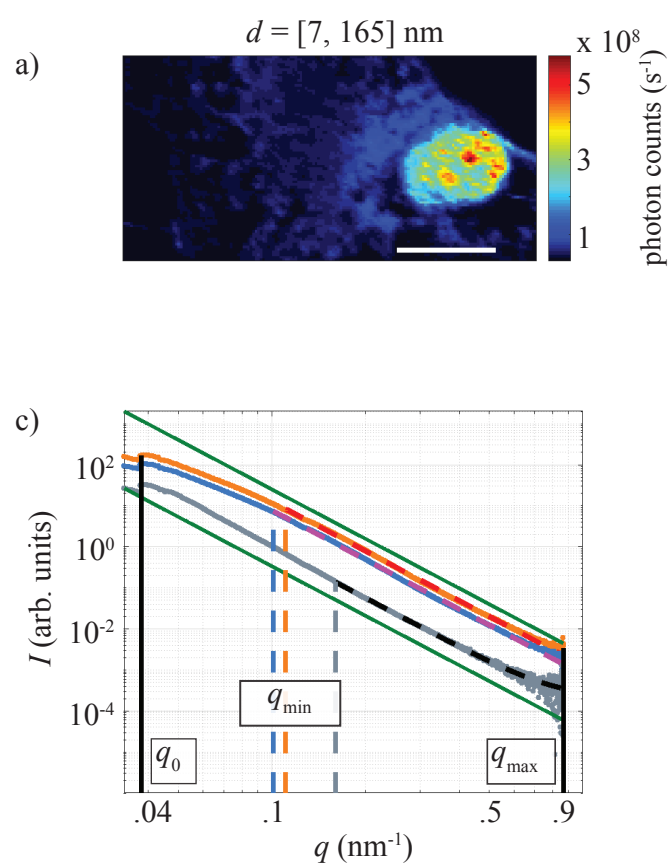

b)

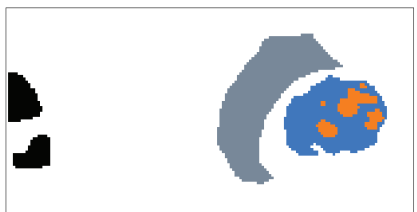

d)

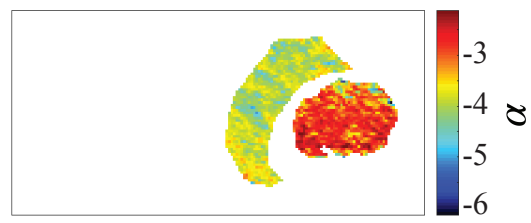

e)

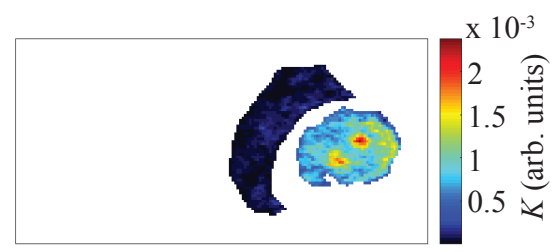

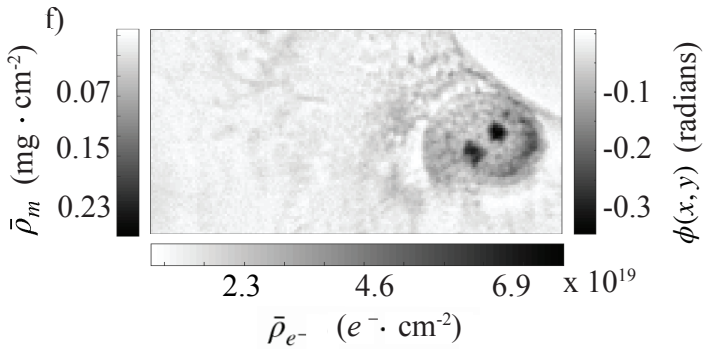

Figure B.12: Sample AW-PD06-C15. The scale bar in a) is $10 \mu \mathrm{m}$ and applies to subfigures b), d), e) and f). See page 117 .

\begin{tabular}{|c|c|c|c|c|c|c|}
\hline Sample & ROI & $\alpha$ & $K$ (arb.units) & $-\phi(\mathrm{rad})$ & $\bar{\rho}_{m}\left(\frac{\mathrm{mg}}{\mathrm{cm}^{2}}\right)$ & $\bar{\rho}_{e^{-}}\left(\frac{\mathrm{e}^{-}}{\mathrm{cm}^{2}}\right)$ \\
\hline \multirow{3}{*}{ AW-PD06-C15 } & Heterochromatin & -2.99 & $1.26 \times 10^{-3}$ & 0.19 & 0.14 & $4.29 \times 10^{19}$ \\
& Euchromatin & -2.95 & $7.76 \times 10^{-4}$ & 0.11 & 0.08 & $2.47 \times 10^{19}$ \\
& Cytoplasm & -3.92 & $6.59 \times 10^{-5}$ & 0.05 & 0.04 & $1.10 \times 10^{19}$ \\
\hline
\end{tabular}


a)
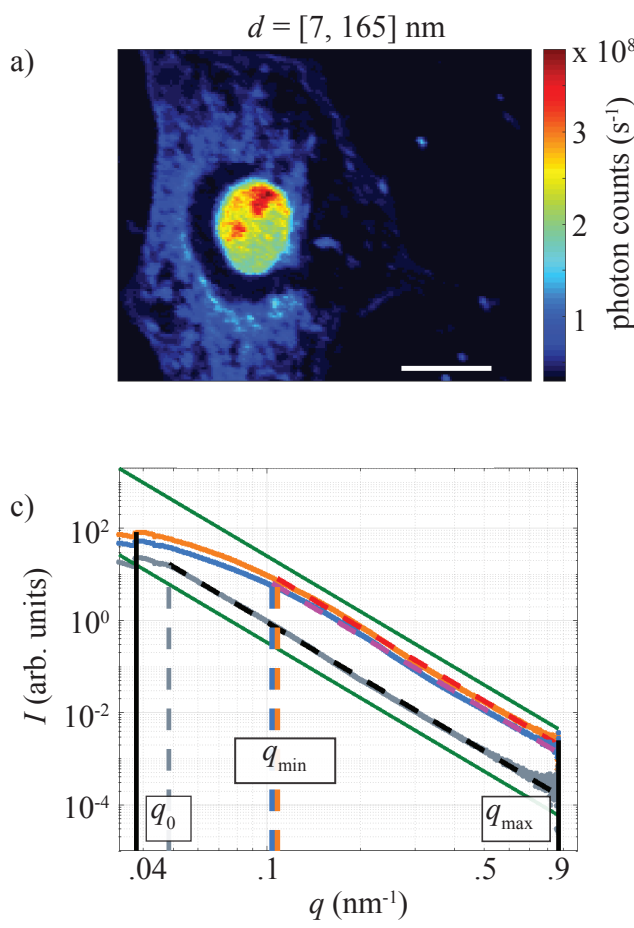

e)

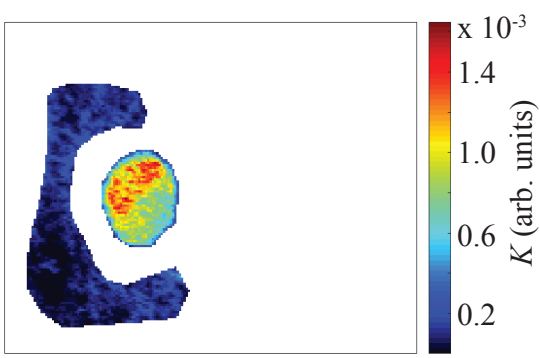

b)

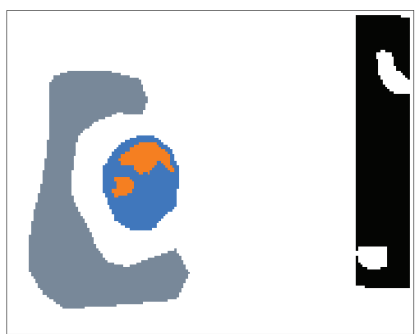

d)
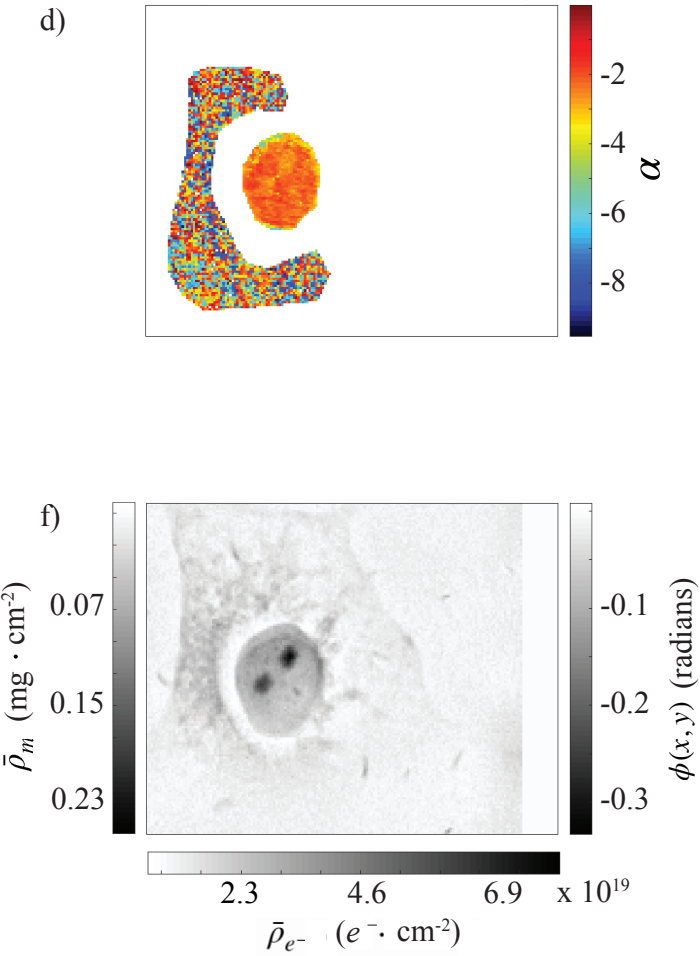

Figure B.13: Sample AW-PD06-C16. The scale bar in a) is $10 \mu \mathrm{m}$ and applies to subfigures b), d), e) and f). In the d) exponent map, the noisy data associated with the cytoplasm is attributed to the small $\left[q_{0}, q_{\mathrm{min}}\right]$ range for this particular cell. See page 117.

\begin{tabular}{|c|c|c|c|c|c|c|}
\hline Sample & ROI & $\alpha$ & $K$ (arb.units) & $-\phi(\mathrm{rad})$ & $\bar{\rho}_{m}\left(\frac{\mathrm{mg}}{\mathrm{cm}^{2}}\right)$ & $\bar{\rho}_{e^{-}}\left(\frac{\mathrm{e}^{-}}{\mathrm{cm}^{2}}\right)$ \\
\hline \multirow{3}{*}{ AW-PD06-C16 } & Heterochromatin & -2.43 & $1.14 \times 10^{-3}$ & 0.16 & 0.12 & $3.65 \times 10^{19}$ \\
& Euchromatin & -2.45 & $7.69 \times 10^{-4}$ & 0.09 & 0.07 & $2.02 \times 10^{19}$ \\
& Cytoplasm & -4.14 & $9.72 \times 10^{-5}$ & 0.03 & 0.03 & $8.00 \times 10^{18}$ \\
\hline
\end{tabular}


a)
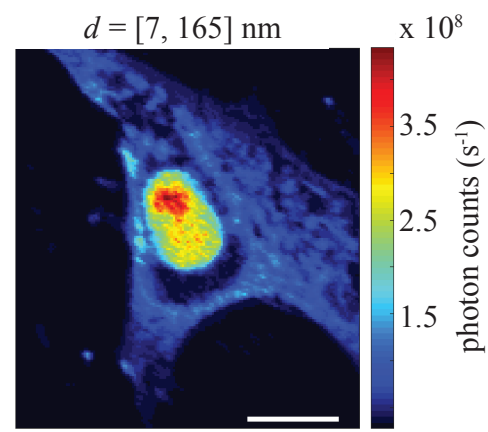

c)

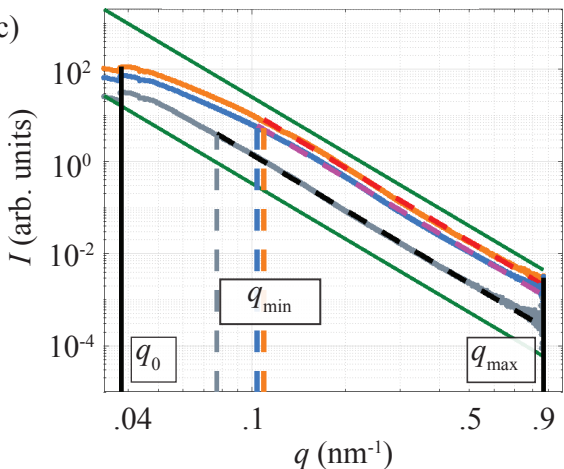

b)

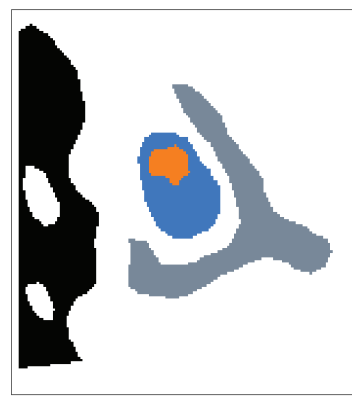

d)
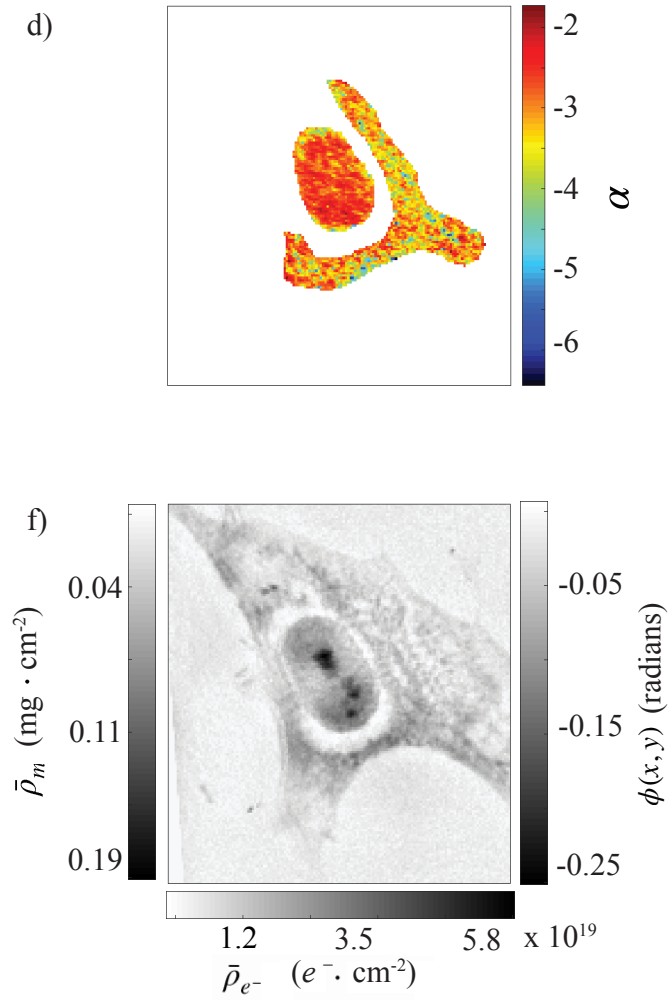

Figure B.14: Sample AW-PD06-C17. The scale bar in a) is $10 \mu \mathrm{m}$ and applies to subfigures b), d), e) and f). See page 117 .

\begin{tabular}{|c|c|c|c|c|c|c|}
\hline Sample & ROI & $\alpha$ & $K$ (arb.units) & $-\phi(\mathrm{rad})$ & $\bar{\rho}_{m}\left(\frac{\mathrm{mg}}{\mathrm{cm}^{2}}\right)$ & $\bar{\rho}_{e^{-}}\left(\frac{\mathrm{e}^{-}}{\mathrm{cm}^{2}}\right)$ \\
\hline \multirow{3}{*}{ AW-PD06-C17 } & Heterochromatin & -2.73 & $1.17 \times 10^{-3}$ & 0.13 & 0.10 & $3.02 \times 10^{19}$ \\
& Euchromatin & -2.81 & $7.13 \times 10^{-4}$ & 0.09 & 0.07 & $2.09 \times 10^{19}$ \\
& Cytoplasm & -3.26 & $1.42 \times 10^{-4}$ & 0.06 & 0.05 & $1.44 \times 10^{19}$ \\
\hline
\end{tabular}


a)

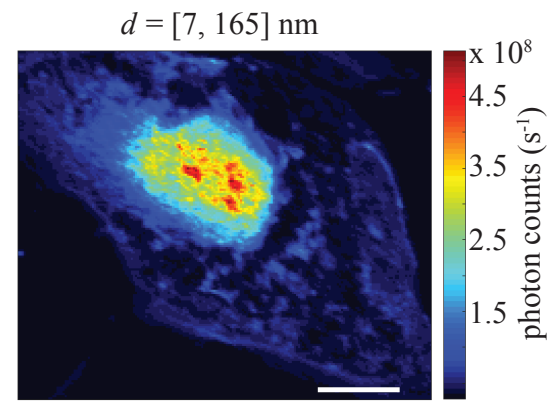

c)

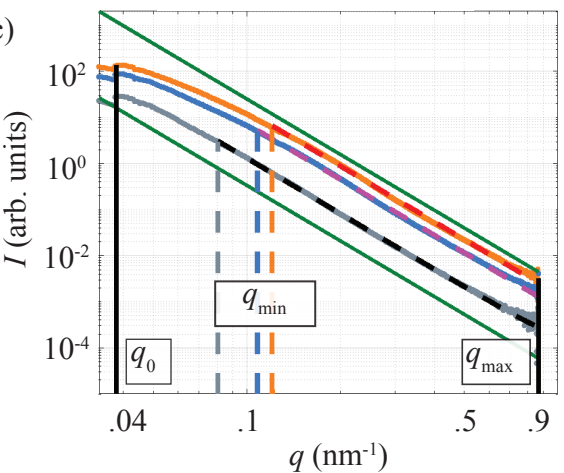

b)

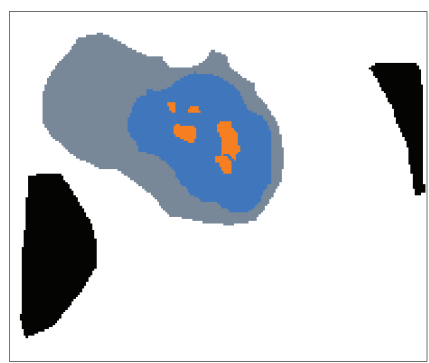

d)

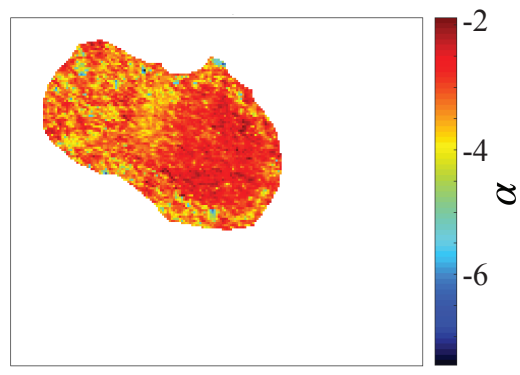

$\checkmark$

\section{.}

e)

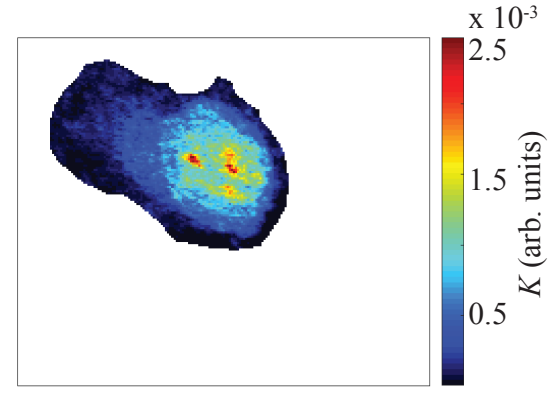

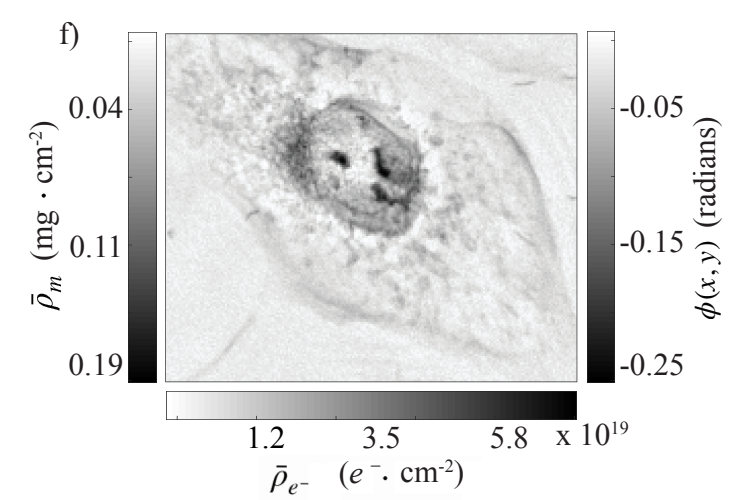

Figure B.15: Sample AW-PD06-C18. The scale bar in a) is $10 \mu \mathrm{m}$ and applies to subfigures b), d), e) and f). See page 117 .

\begin{tabular}{|c|c|c|c|c|c|c|}
\hline Sample & ROI & $\alpha$ & $K$ (arb.units) & $-\phi(\mathrm{rad})$ & $\bar{\rho}_{m}\left(\frac{\mathrm{mg}}{\mathrm{cm}^{2}}\right)$ & $\bar{\rho}_{e^{-}}\left(\frac{\mathrm{e}^{-}}{\mathrm{cm}^{2}}\right)$ \\
\hline \multirow{3}{*}{ AW-PD06-C18 } & Heterochromatin & -2.81 & $1.40 \times 10^{-3}$ & 0.13 & 0.10 & $2.93 \times 10^{19}$ \\
& Euchromatin & -2.91 & $7.12 \times 10^{-4}$ & 0.08 & 0.06 & $1.84 \times 10^{19}$ \\
& Cytoplasm & -3.33 & $1.27 \times 10^{-4}$ & 0.03 & 0.03 & $7.85 \times 10^{18}$ \\
\hline
\end{tabular}


a)

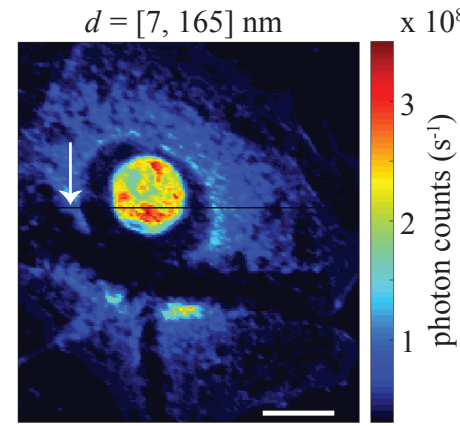

c)

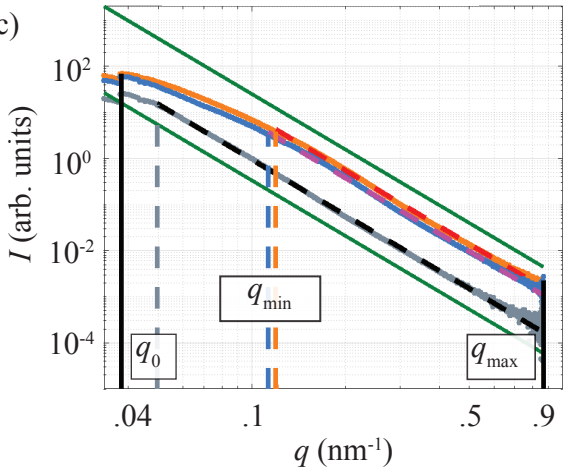

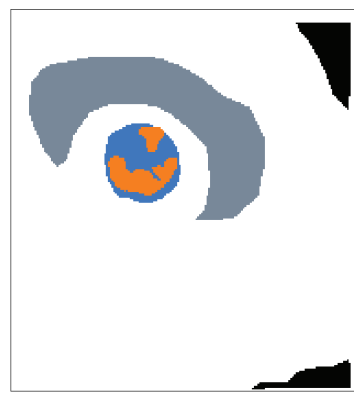

e)

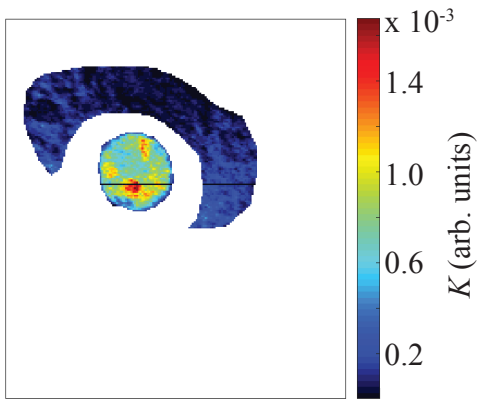

b)

d)
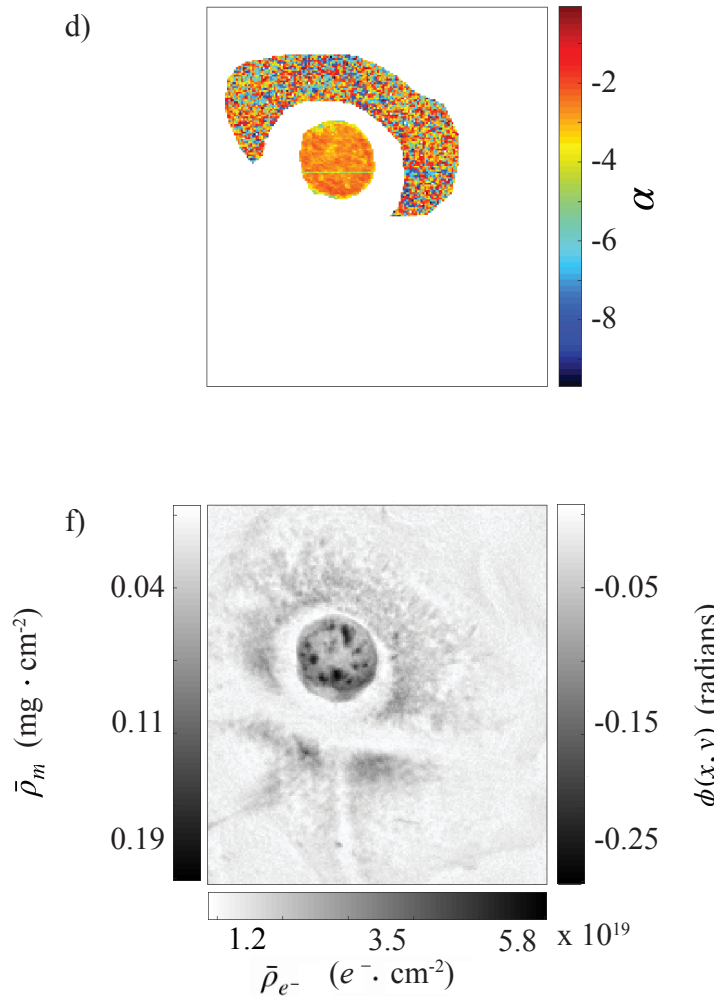

Figure B.16: Sample AW-PD06-C19. During the scanning SAXS measurement, a row of data was not collected. This row is indicated by the white arrow in a). The scale bar in a) is $10 \mu \mathrm{m}$ and applies to subfigures b), d), e) and f). In the d) exponent map, the noisy data associated with the cytoplasm is attributed to the small $\left[q_{0}, q_{\mathrm{min}}\right]$ range for this particular cell. See page 117 .

\begin{tabular}{|c|c|c|c|c|c|c|}
\hline Sample & ROI & $\alpha$ & $K$ (arb.units) & $-\phi(\mathrm{rad})$ & $\bar{\rho}_{m}\left(\frac{\mathrm{mg}}{\mathrm{cm}^{2}}\right)$ & $\bar{\rho}_{e^{-}}\left(\frac{\mathrm{e}^{-}}{\mathrm{cm}^{2}}\right)$ \\
\hline \multirow{3}{*}{ AW-PD06-C19 } & Heterochromatin & -2.75 & $8.81 \times 10^{-4}$ & 0.15 & 0.11 & $3.44 \times 10^{19}$ \\
& Euchromatin & -2.88 & $6.13 \times 10^{-4}$ & 0.10 & 0.08 & $2.30 \times 10^{19}$ \\
& Cytoplasm & -3.94 & $1.01 \times 10^{-4}$ & 0.04 & 0.03 & $8.94 \times 10^{18}$ \\
\hline
\end{tabular}


a)
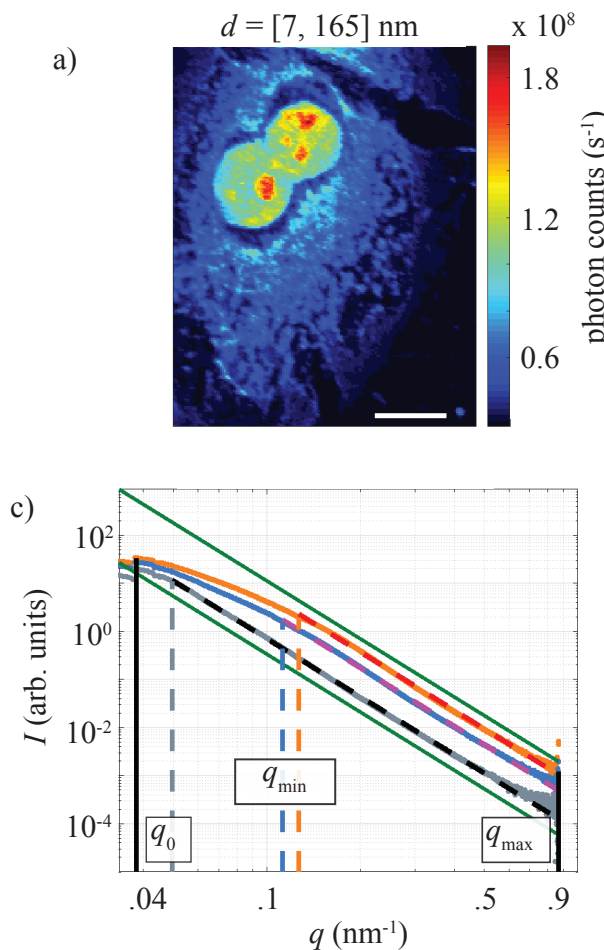

e)

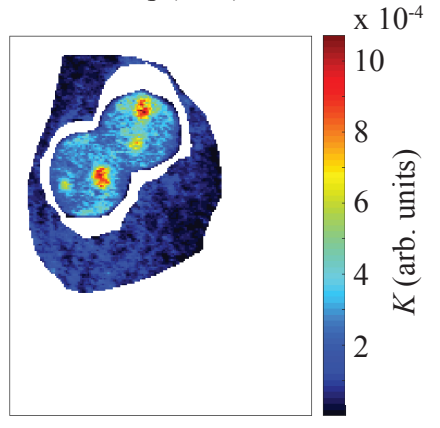

b)

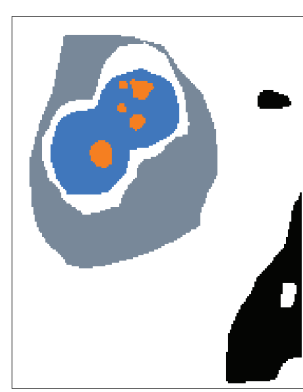

d)
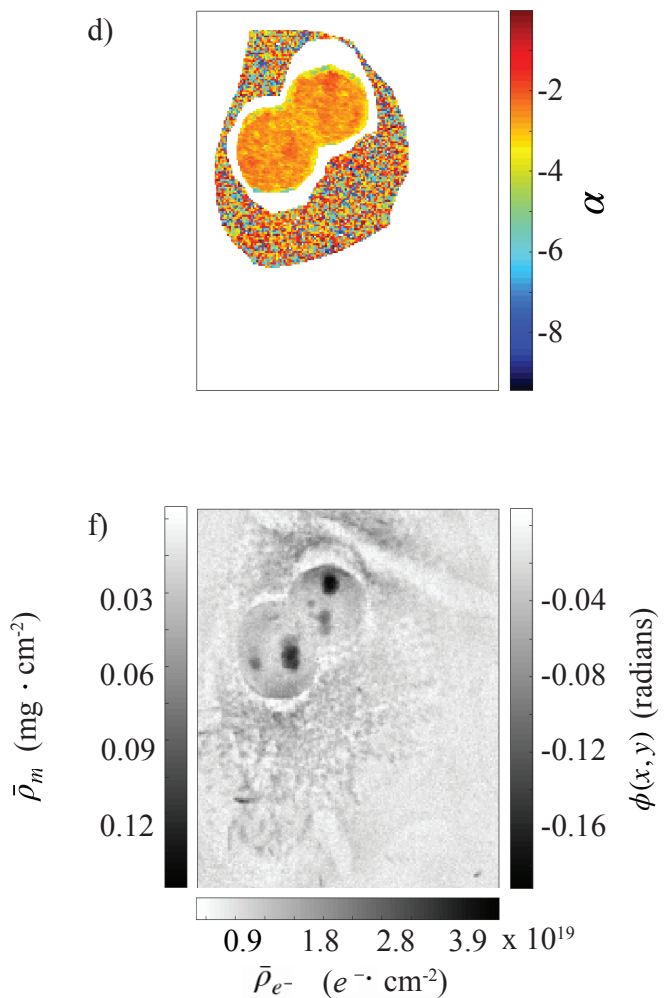

Figure B.17: Sample AW-PD06-C20. The scale bar in a) is $10 \mu \mathrm{m}$ and applies to subfigures b), d), e) and $\mathrm{f}$ ). In the d) exponent map, the noisy data associated with the cytoplasm is attributed to the small $\left[q_{0}, q_{\text {min }}\right]$ range for this particular cell. See page 117.

\begin{tabular}{|c|c|c|c|c|c|c|}
\hline Sample & ROI & $\alpha$ & $K$ (arb.units) & $-\phi(\mathrm{rad})$ & $\bar{\rho}_{m}\left(\frac{\mathrm{mg}}{\mathrm{cm}^{2}}\right)$ & $\bar{\rho}_{e^{-}}\left(\frac{\mathrm{e}^{-}}{\mathrm{cm}^{2}}\right)$ \\
\hline \multirow{3}{*}{ AW-PD06-C20 } & Heterochromatin & -2.47 & $5.92 \times 10^{-4}$ & 0.10 & 0.08 & $2.36 \times 10^{19}$ \\
& Euchromatin & -2.84 & $2.74 \times 10^{-4}$ & 0.04 & 0.03 & $9.48 \times 10^{18}$ \\
& Cytoplasm & -3.65 & $7.30 \times 10^{-5}$ & 0.03 & 0.02 & $7.12 \times 10^{18}$ \\
\hline
\end{tabular}


a)

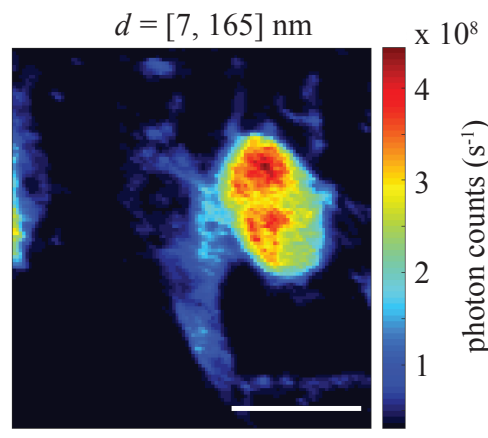

c)

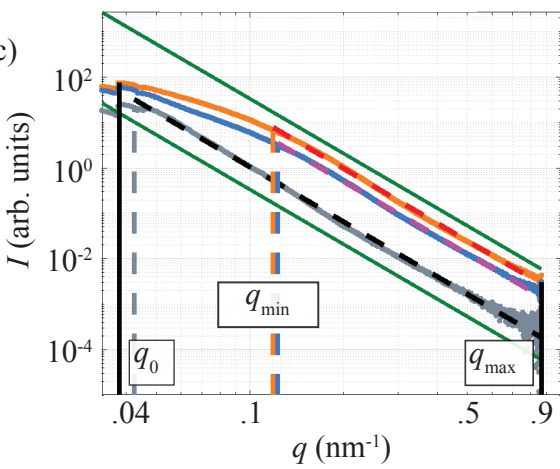

e)

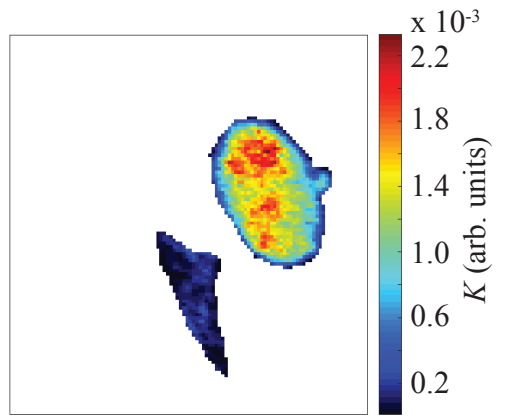

b)

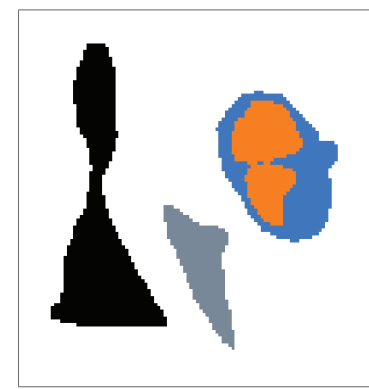

d)
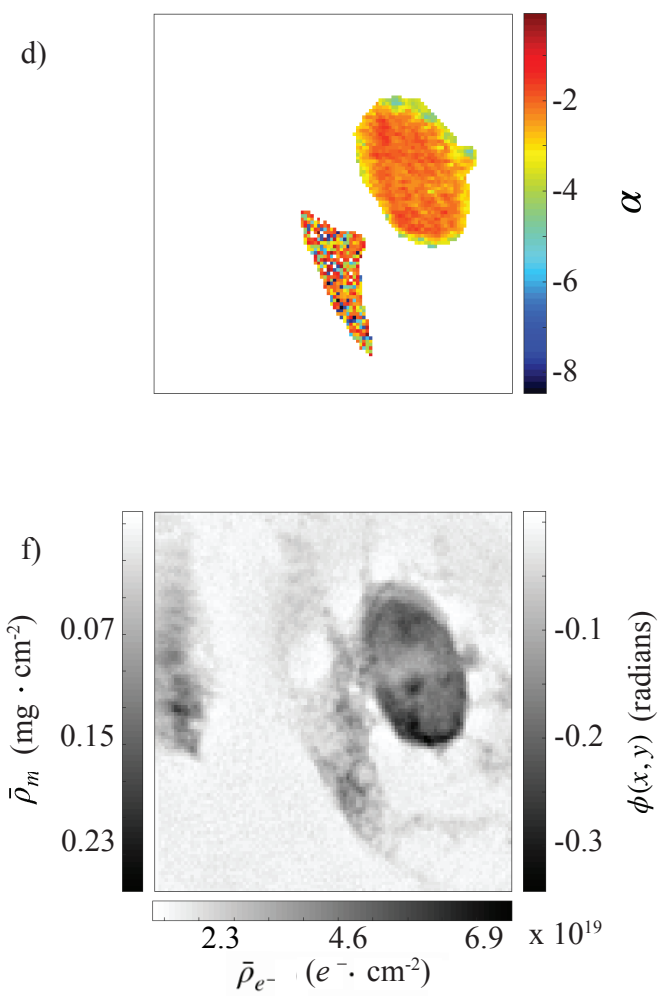

Figure B.18: Sample AW-PD06-C22. The scale bar in a) is $10 \mu \mathrm{m}$ and applies to subfigures b), d), e) and f). In the d) exponent map, the noisy data associated with the cytoplasm is attributed to the small $\left[q_{0}, q_{\text {min }}\right]$ range for this particular cell. See page 117.

\begin{tabular}{|c|c|c|c|c|c|c|}
\hline Sample & ROI & $\alpha$ & $K$ (arb.units) & $-\phi(\mathrm{rad})$ & $\bar{\rho}_{m}\left(\frac{\mathrm{mg}}{\mathrm{cm}^{2}}\right)$ & $\bar{\rho}_{e^{-}}\left(\frac{\mathrm{e}^{-}}{\mathrm{cm}^{2}}\right)$ \\
\hline \multirow{3}{*}{ AW-PD06-C22 } & Heterochromatin & -2.20 & $1.57 \times 10^{-3}$ & 0.18 & 0.14 & $4.18 \times 10^{19}$ \\
& Euchromatin & -2.74 & $7.96 \times 10^{-4}$ & 0.17 & 0.13 & $3.90 \times 10^{19}$ \\
& Cytoplasm & -3.20 & $1.08 \times 10^{-4}$ & 0.08 & 0.06 & $1.91 \times 10^{19}$ \\
\hline
\end{tabular}


a)
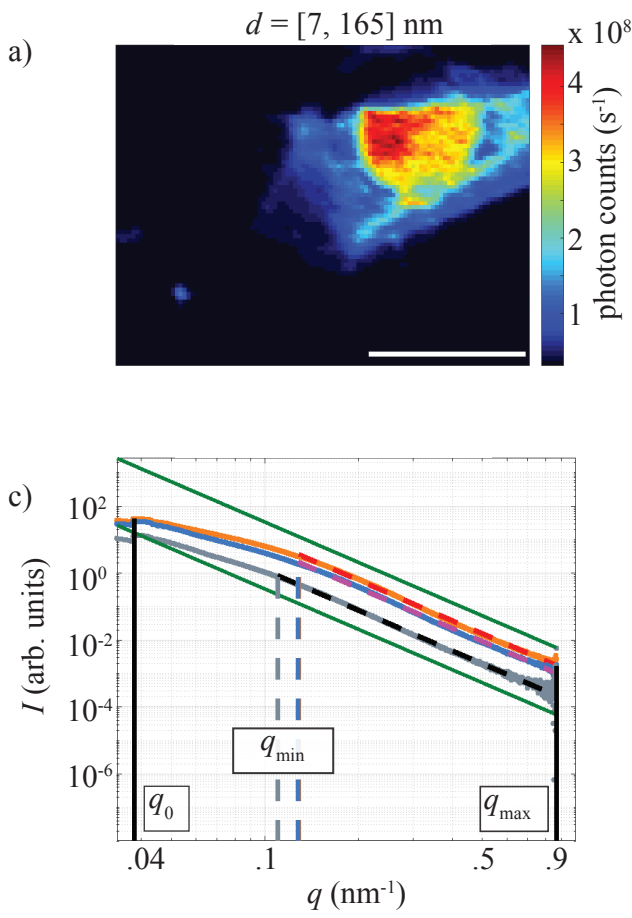

e)

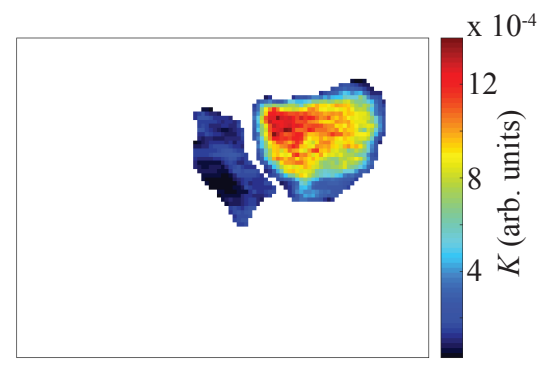

b)

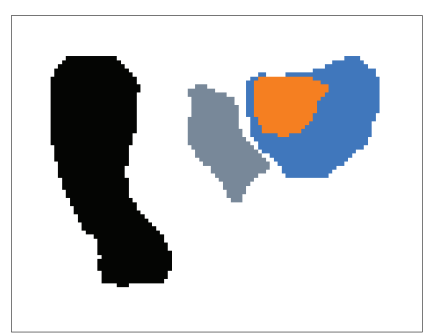

d)
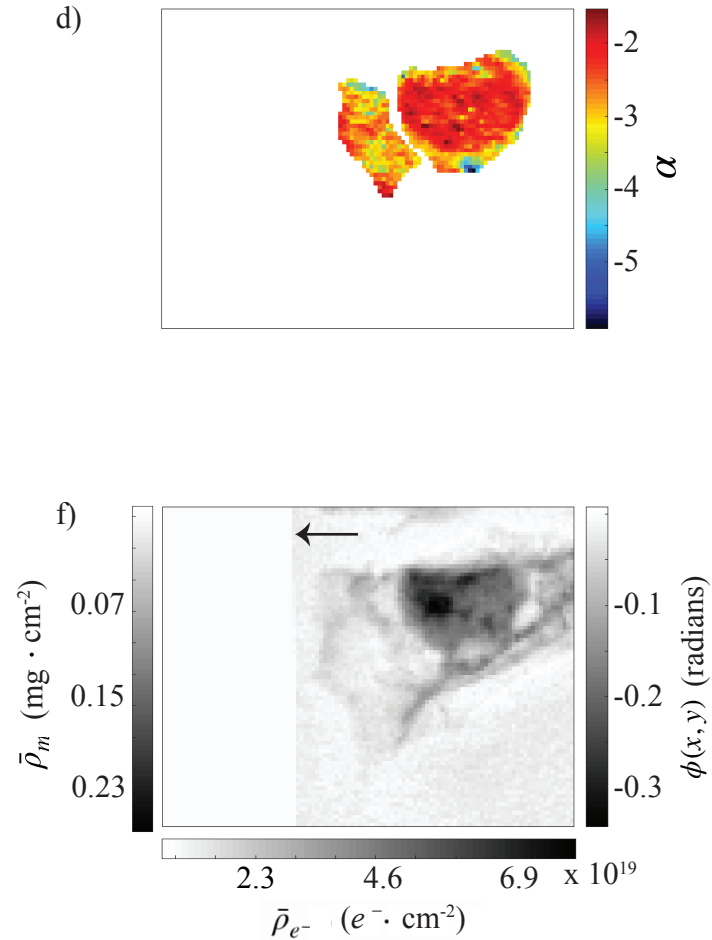

Figure B.19: Sample AW-PD06-C23. The scale bar in a) is $10 \mu \mathrm{m}$ and applies to subfigures b), d), e) and f). In f), the black arrow indicates a region where X-ray holography data was not collected. See page 117 .

\begin{tabular}{|c|c|c|c|c|c|c|}
\hline Sample & ROI & $\alpha$ & $K$ (arb.units) & $-\phi(\mathrm{rad})$ & $\bar{\rho}_{m}\left(\frac{\mathrm{mg}}{\mathrm{cm}^{2}}\right)$ & $\bar{\rho}_{e^{-}}\left(\frac{\mathrm{e}^{-}}{\mathrm{cm}^{2}}\right)$ \\
\hline \multirow{3}{*}{ AW-PD06-C23 } & Heterochromatin & -2.24 & $9.85 \times 10^{-4}$ & 0.24 & 0.18 & $5.47 \times 10^{19}$ \\
& Euchromatin & -2.59 & $5.71 \times 10^{-4}$ & 0.14 & 0.11 & $3.24 \times 10^{19}$ \\
& Cytoplasm & -2.85 & $1.21 \times 10^{-4}$ & 0.04 & 0.03 & $8.94 \times 10^{18}$ \\
\hline
\end{tabular}


a)

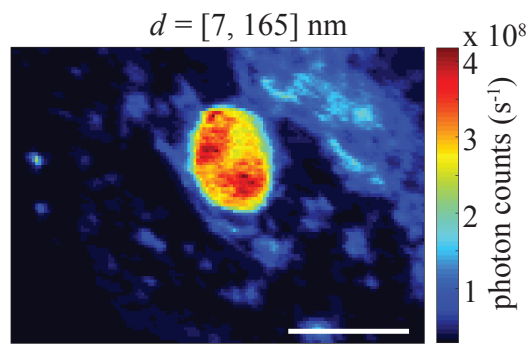

c)

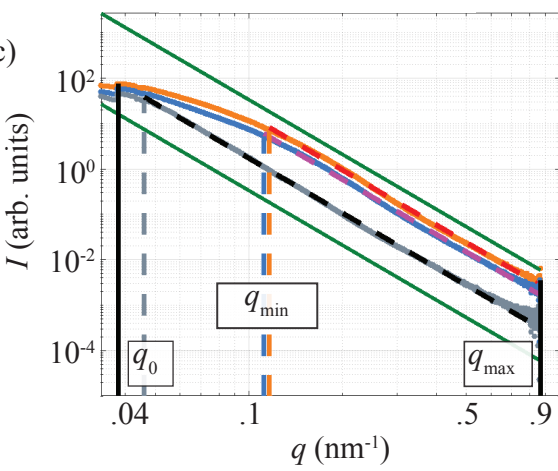

e)

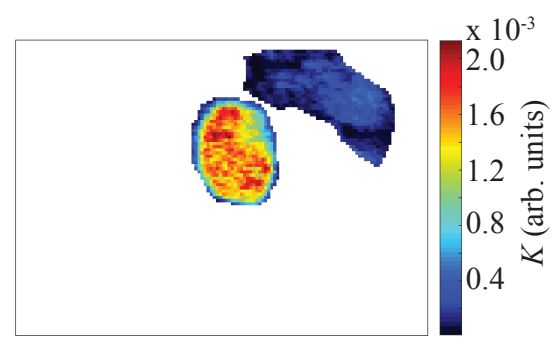

b)

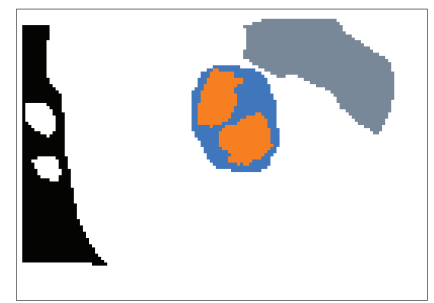

d)
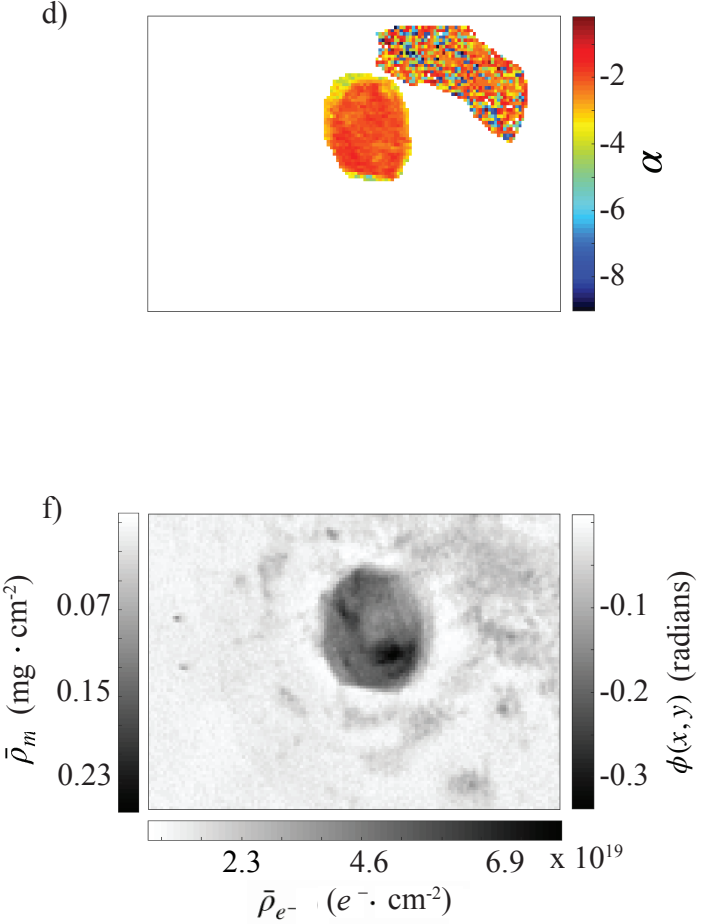

Figure B.20: Sample AW-PD06-C24. The scale bar in a) is $10 \mu \mathrm{m}$ and applies to subfigures b), d), e) and f). In the d) exponent map, the noisy data associated with the cytoplasm is attributed to the small $\left[q_{0}, q_{\text {min }}\right]$ range for this particular cell. See page 117.

\begin{tabular}{|c|c|c|c|c|c|c|}
\hline Sample & ROI & $\alpha$ & $K$ (arb.units) & $-\phi(\mathrm{rad})$ & $\bar{\rho}_{m}\left(\frac{\mathrm{mg}}{\mathrm{cm}^{2}}\right)$ & $\bar{\rho}_{e^{-}}\left(\frac{\mathrm{e}^{-}}{\mathrm{cm}^{2}}\right)$ \\
\hline \multirow{3}{*}{ AW-PD06-C24 } & Heterochromatin & -2.18 & $1.53 \times 10^{-3}$ & 0.21 & 0.16 & $4.88 \times 10^{19}$ \\
& Euchromatin & -2.51 & $9.36 \times 10^{-4}$ & 0.18 & 0.13 & $4.04 \times 10^{19}$ \\
& Cytoplasm & -3.43 & $1.78 \times 10^{-4}$ & 0.05 & 0.04 & $1.18 \times 10^{19}$ \\
\hline
\end{tabular}


a)
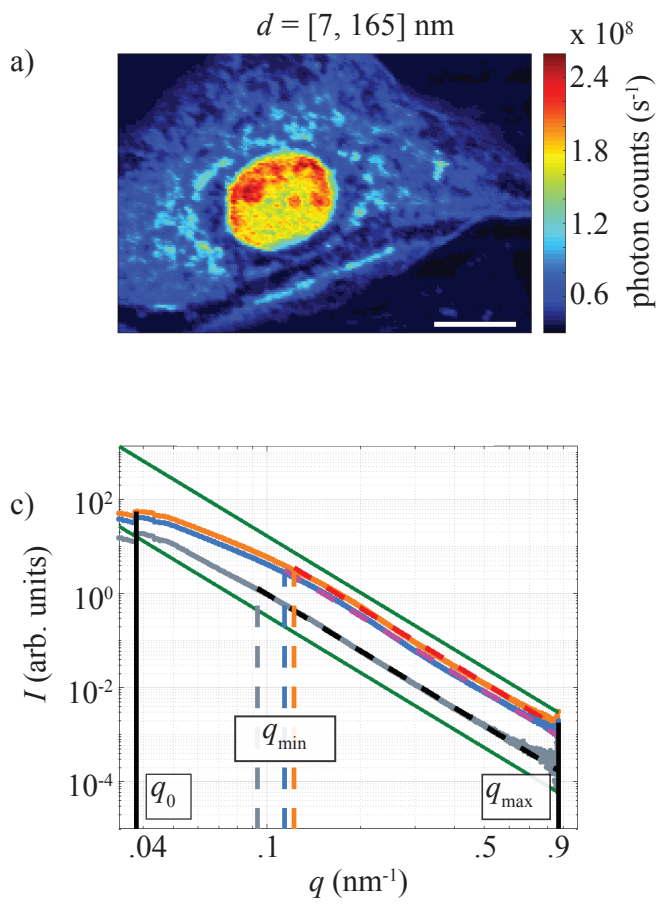

e)

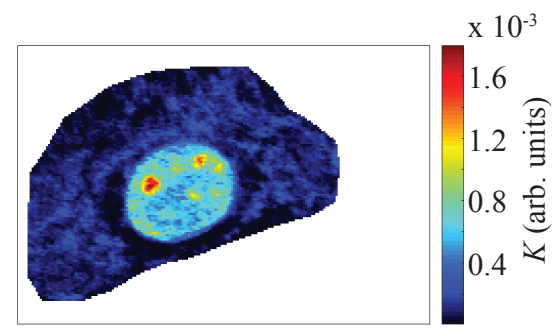

b)

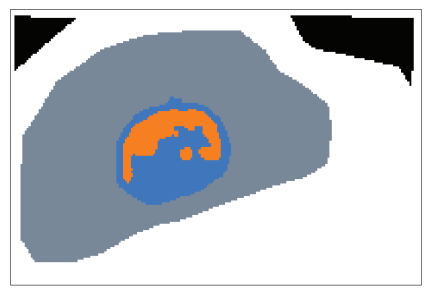

d)

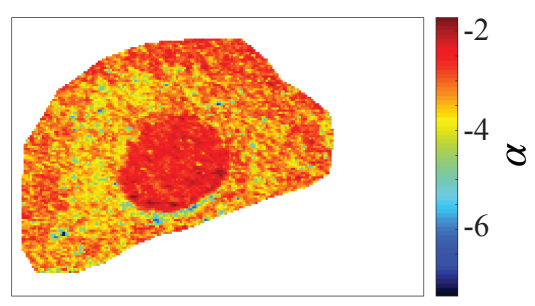

Figure B.21: Sample AW-PD06-C26. The scale bar in a) is $10 \mu \mathrm{m}$ and applies to subfigures b), d), e) and f). See page 117 .

\begin{tabular}{|c|c|c|c|c|c|c|}
\hline Sample & ROI & $\alpha$ & $K$ (arb.units) & $-\phi(\mathrm{rad})$ & $\bar{\rho}_{m}\left(\frac{\mathrm{mg}}{\mathrm{cm}^{2}}\right)$ & $\bar{\rho}_{e^{-}}\left(\frac{\mathrm{e}^{-}}{\mathrm{cm}^{2}}\right)$ \\
\hline \multirow{3}{*}{ AW-PD06-C26 } & Heterochromatin & -2.61 & $7.94 \times 10^{-4}$ & 0.11 & 0.09 & $2.62 \times 10^{19}$ \\
& Euchromatin & -2.69 & $5.30 \times 10^{-4}$ & 0.07 & 0.05 & $1.65 \times 10^{19}$ \\
& Cytoplasm & -3.31 & $9.21 \times 10^{-5}$ & 0.04 & 0.03 & $8.55 \times 10^{18}$ \\
\hline
\end{tabular}


a)
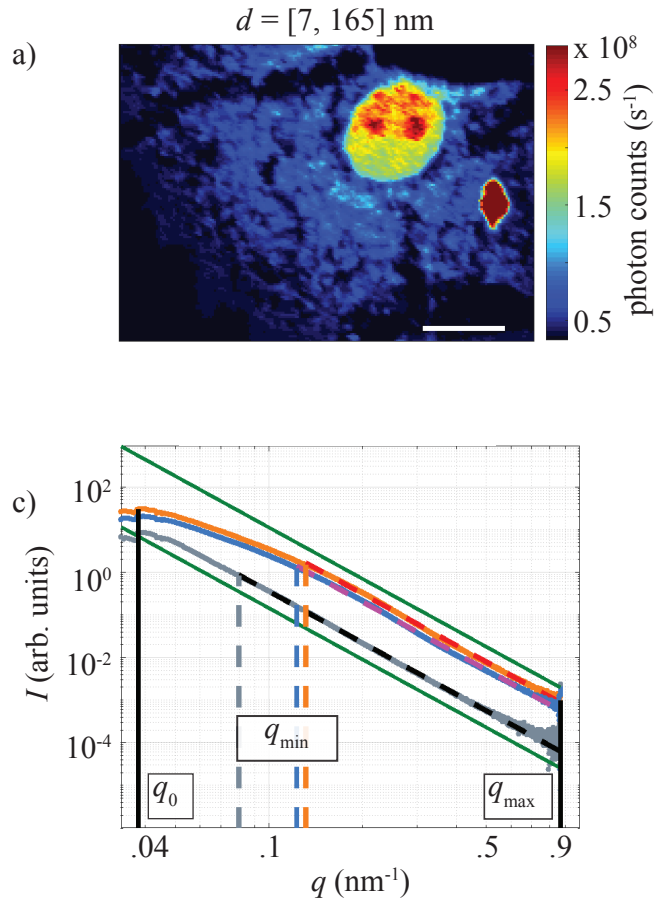

e)

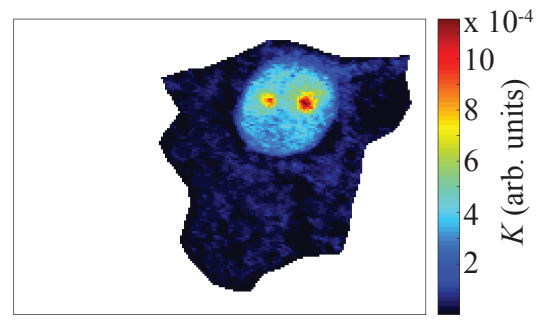

b)

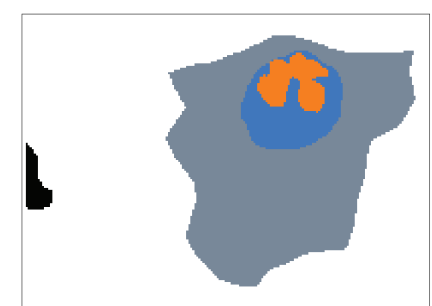

d)
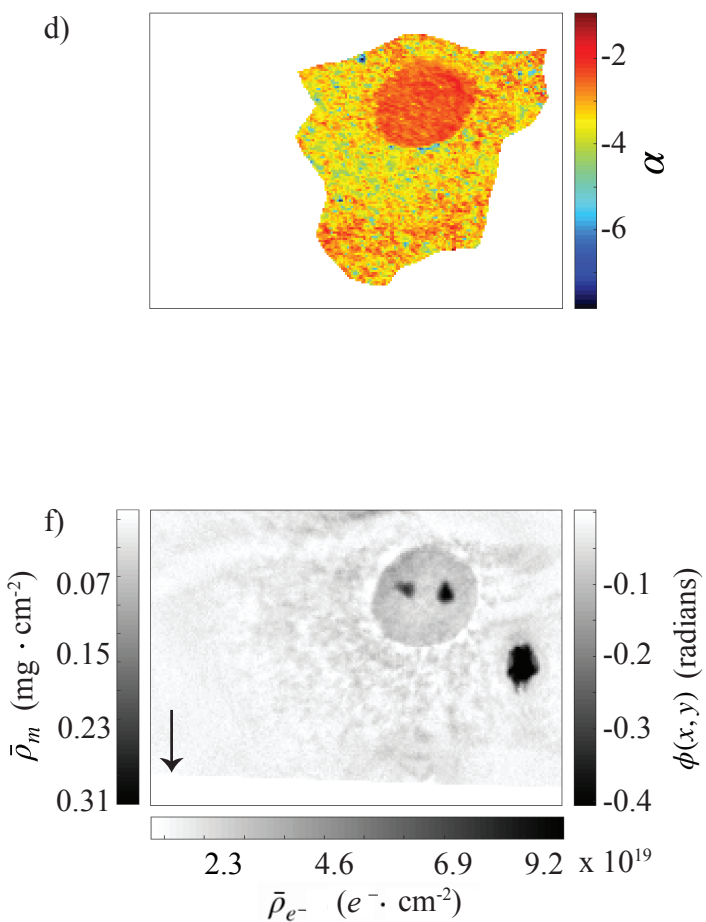

Figure B.22: Sample AW-PD06-C27. The scale bar in a) is $10 \mu \mathrm{m}$ and applies to subfigures b), d), e) and f). In $\mathrm{f}$ ), the black arrow indicates a region where X-ray holography data was not collected. See page 117 .

\begin{tabular}{|c|c|c|c|c|c|c|}
\hline Sample & ROI & $\alpha$ & $K$ (arb.units) & $-\phi(\mathrm{rad})$ & $\bar{\rho}_{m}\left(\frac{\mathrm{mg}}{\mathrm{cm}^{2}}\right)$ & $\bar{\rho}_{e^{-}}\left(\frac{\mathrm{e}^{-}}{\mathrm{cm}^{2}}\right)$ \\
\hline \multirow{3}{*}{ AW-PD06-C27 } & Heterochromatin & -2.58 & $4.93 \times 10^{-4}$ & 0.13 & 0.10 & $3.07 \times 10^{19}$ \\
& Euchromatin & -2.57 & $3.21 \times 10^{-4}$ & 0.08 & 0.06 & $1.83 \times 10^{19}$ \\
& Cytoplasm & -3.36 & $3.26 \times 10^{-5}$ & 0.03 & 0.03 & $8.03 \times 10^{18}$ \\
\hline
\end{tabular}


a)

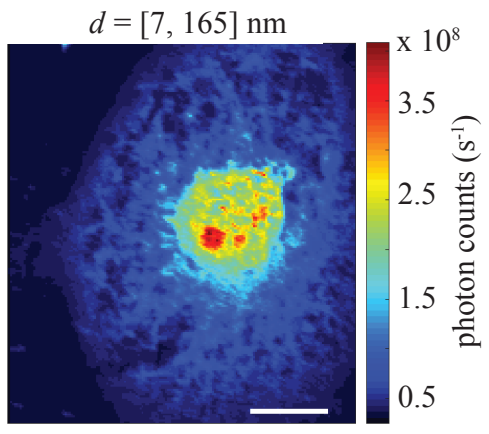

c)

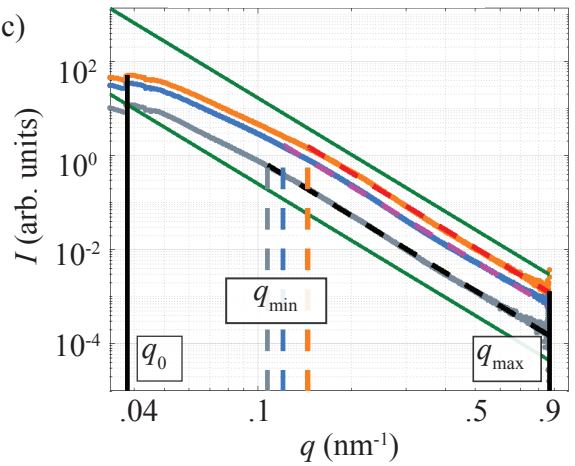

b)

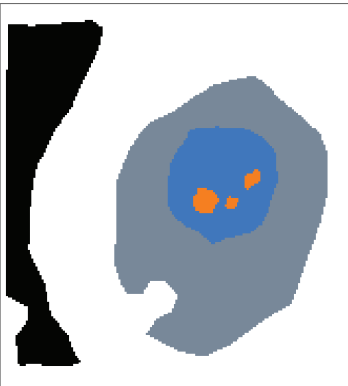

d)
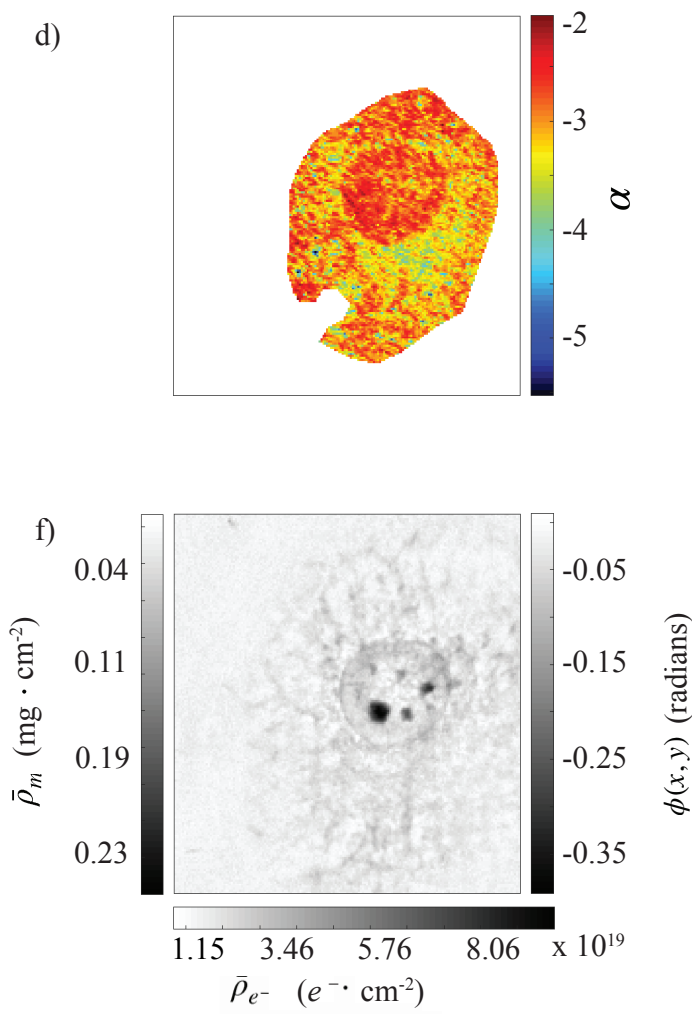

Figure B.23: Sample AW-PD06-C29. The scale bar in a) is $10 \mu \mathrm{m}$ and applies to subfigures b), d), e) and f). See page 117.

\begin{tabular}{|c|c|c|c|c|c|c|}
\hline Sample & ROI & $\alpha$ & $K$ (arb.units) & $-\phi(\mathrm{rad})$ & $\bar{\rho}_{m}\left(\frac{\mathrm{mg}}{\mathrm{cm}^{2}}\right)$ & $\bar{\rho}_{e^{-}}\left(\frac{\mathrm{e}^{-}}{\mathrm{cm}^{2}}\right)$ \\
\hline \multirow{3}{*}{ AW-PD06-C29 } & Heterochromatin & -2.85 & $6.83 \times 10^{-4}$ & 0.20 & 0.15 & $4.50 \times 10^{19}$ \\
& Euchromatin & -2.86 & $3.53 \times 10^{-4}$ & 0.06 & 0.05 & $1.40 \times 10^{19}$ \\
& Cytoplasm & -3.08 & $7.94 \times 10^{-5}$ & 0.03 & 0.03 & $7.83 \times 10^{18}$ \\
\hline
\end{tabular}


a)

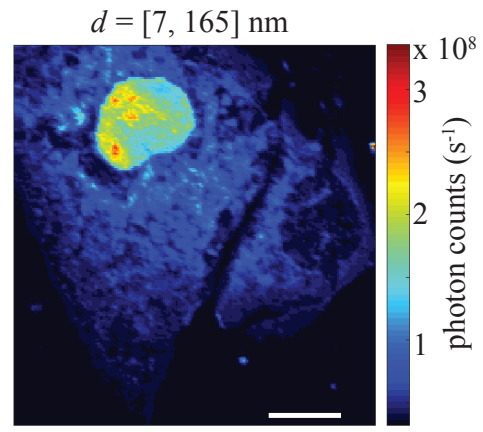

c)

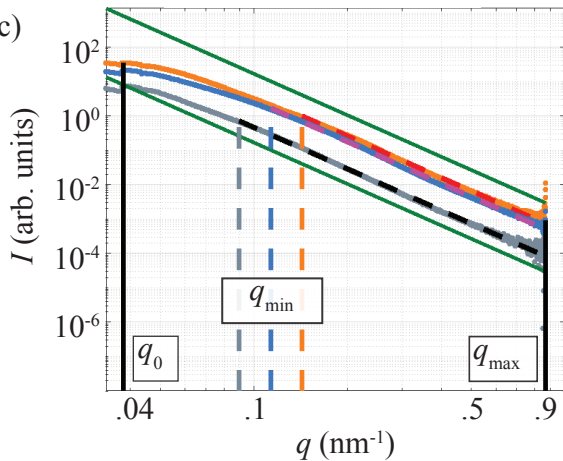

b)

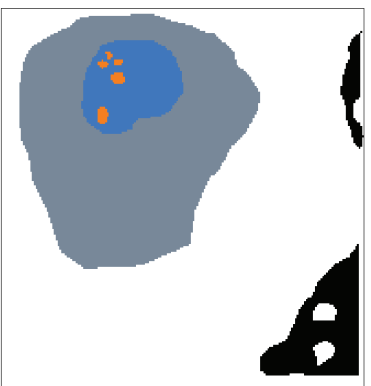

d)
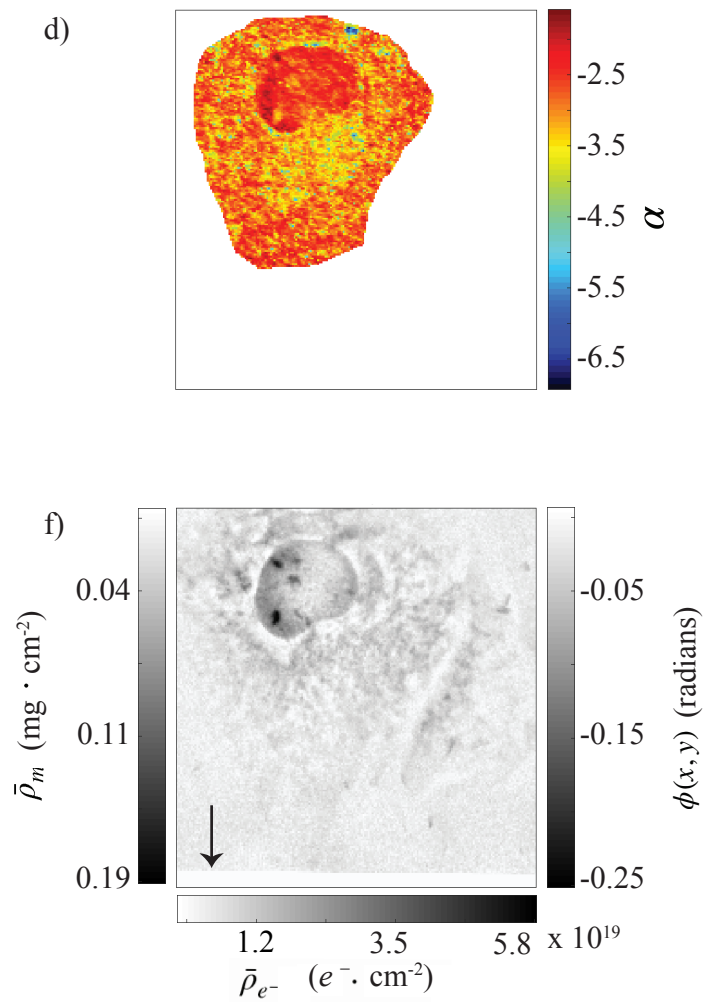

Figure B.24: Sample AW-PD06-C30. The scale bar in a) is $10 \mu \mathrm{m}$ and applies to subfigures $\mathrm{b}$ ), d), e) and $\mathrm{f}$ ). In $\mathrm{f}$ ), the black arrow indicates a region where X-ray holography data was not collected. See page 117 .

\begin{tabular}{|c|c|c|c|c|c|c|}
\hline Sample & ROI & $\alpha$ & $K$ (arb.units) & $-\phi(\mathrm{rad})$ & $\bar{\rho}_{m}\left(\frac{\mathrm{mg}}{\mathrm{cm}^{2}}\right)$ & $\bar{\rho}_{e^{-}}\left(\frac{\mathrm{e}^{-}}{\mathrm{cm}^{2}}\right)$ \\
\hline \multirow{3}{*}{ AW-PD06-C30 } & Heterochromatin & -2.91 & $4.08 \times 10^{-4}$ & 0.14 & 0.11 & $3.29 \times 10^{19}$ \\
& Euchromatin & -2.59 & $2.81 \times 10^{-4}$ & 0.06 & 0.05 & $1.38 \times 10^{19}$ \\
& Cytoplasm & -3.01 & $4.34 \times 10^{-5}$ & 0.03 & 0.02 & $6.78 \times 10^{18}$ \\
\hline
\end{tabular}


a)
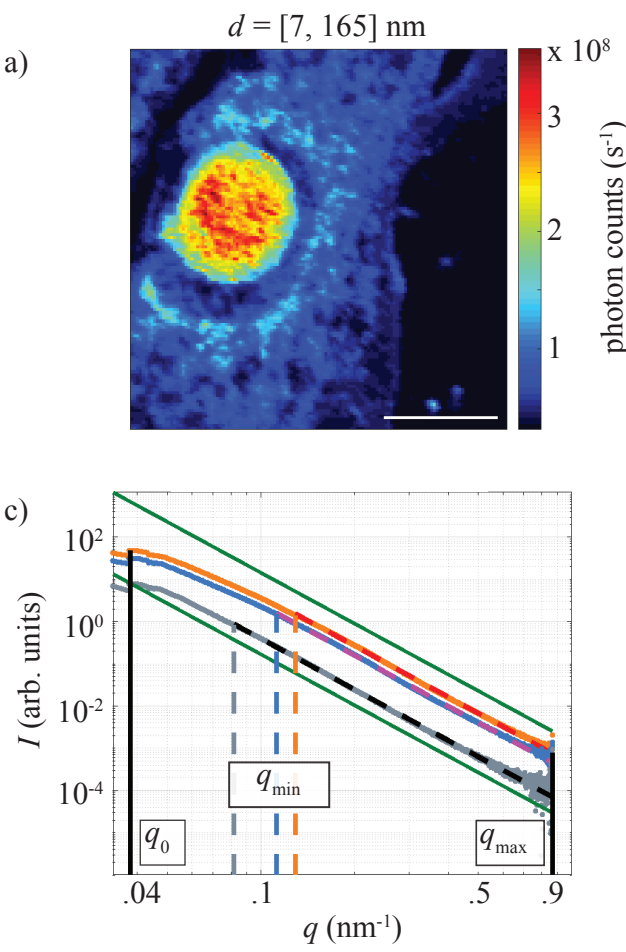

e)

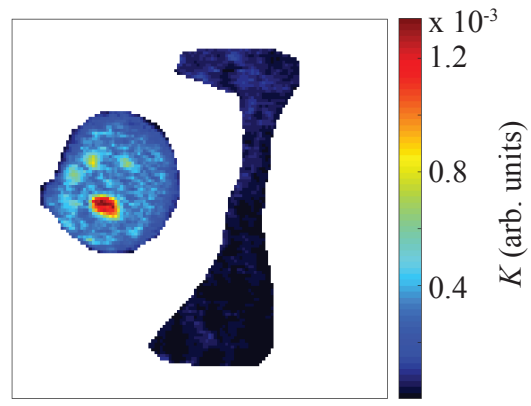

b)

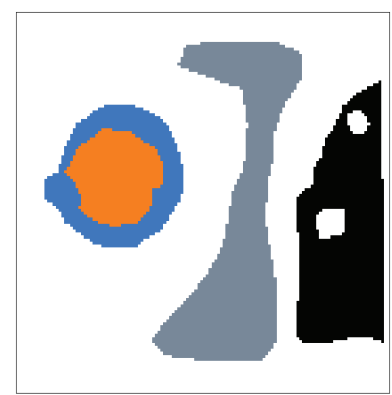

d)
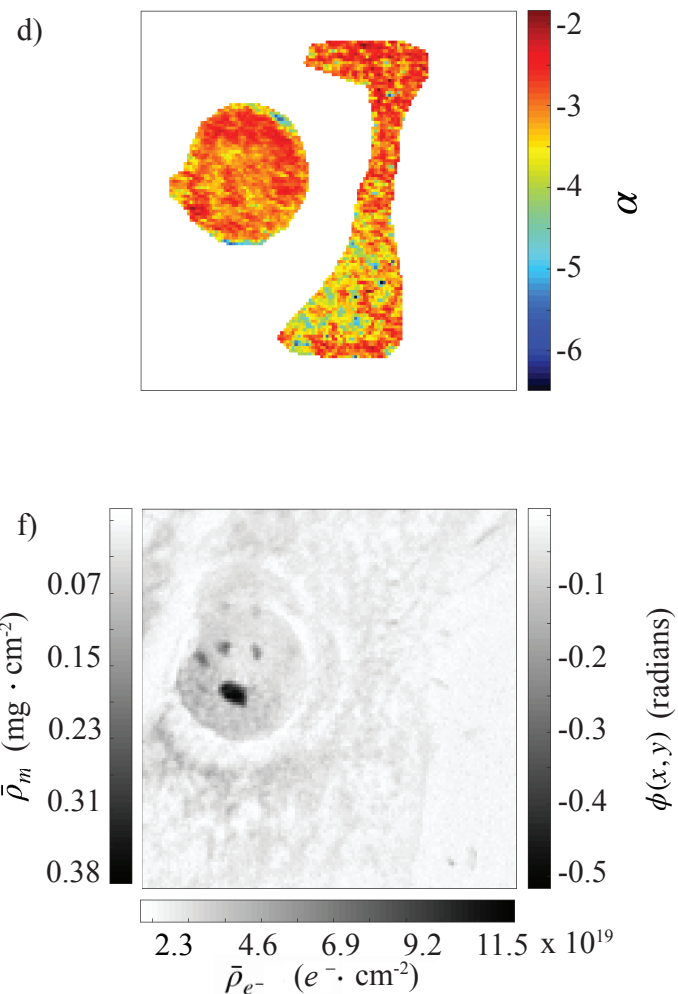

Figure B.25: Sample AW-PD06-C31. The scale bar in a) is $10 \mu \mathrm{m}$ and applies to subfigures b), d), e) and f). See page 117 .

\begin{tabular}{|c|c|c|c|c|c|c|}
\hline Sample & ROI & $\alpha$ & $K$ (arb.units) & $-\phi(\mathrm{rad})$ & $\bar{\rho}_{m}\left(\frac{\mathrm{mg}}{\mathrm{cm}^{2}}\right)$ & $\bar{\rho}_{e^{-}}\left(\frac{\mathrm{e}^{-}}{\mathrm{cm}^{2}}\right)$ \\
\hline \multirow{3}{*}{ AW-PD06-C31 } & Heterochromatin & -3.01 & $4.19 \times 10^{-4}$ & 0.11 & 0.08 & $2.54 \times 10^{19}$ \\
& Euchromatin & -3.05 & $2.48 \times 10^{-4}$ & 0.07 & 0.05 & $1.60 \times 10^{19}$ \\
& Cytoplasm & -3.17 & $3.65 \times 10^{-5}$ & 0.03 & 0.02 & $6.80 \times 10^{18}$ \\
\hline
\end{tabular}


a)
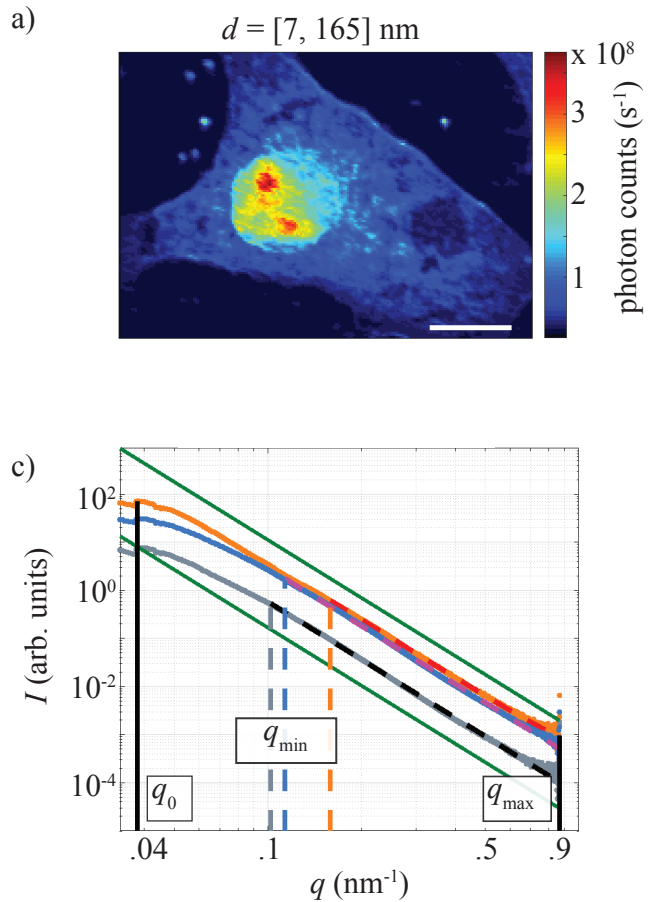

e)

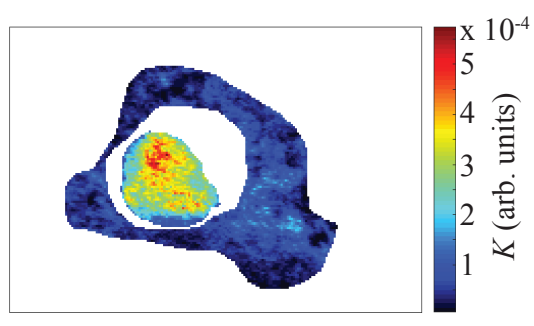

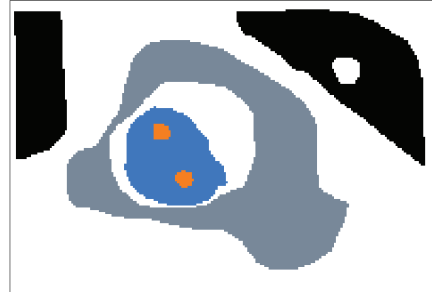

d)

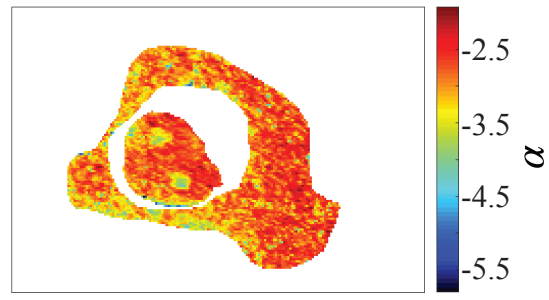

f)

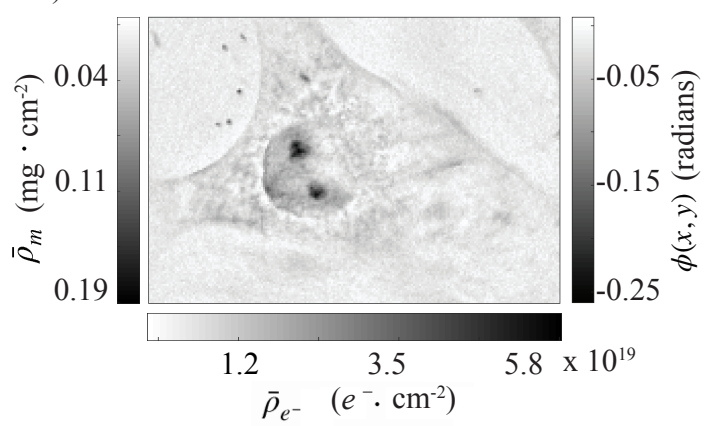

Figure B.26: Sample AW-PD06-C35. The scale bar in a) is $10 \mu \mathrm{m}$ and applies to subfigures b), d), e) and f). See page 117 .

\begin{tabular}{|c|c|c|c|c|c|c|}
\hline Sample & ROI & $\alpha$ & $K$ (arb.units) & $-\phi(\mathrm{rad})$ & $\bar{\rho}_{m}\left(\frac{\mathrm{mg}}{\mathrm{cm}^{2}}\right)$ & $\bar{\rho}_{e^{-}}\left(\frac{\mathrm{e}^{-}}{\mathrm{cm}^{2}}\right)$ \\
\hline \multirow{3}{*}{ AW-PD06-C35 } & Heterochromatin & -3.51 & $3.76 \times 10^{-4}$ & 0.16 & 0.12 & $3.60 \times 10^{19}$ \\
& Euchromatin & -2.84 & $2.86 \times 10^{-4}$ & 0.06 & 0.04 & $1.35 \times 10^{19}$ \\
& Cytoplasm & -2.91 & $5.81 \times 10^{-5}$ & 0.03 & 0.02 & $6.55 \times 10^{18}$ \\
\hline
\end{tabular}


a)

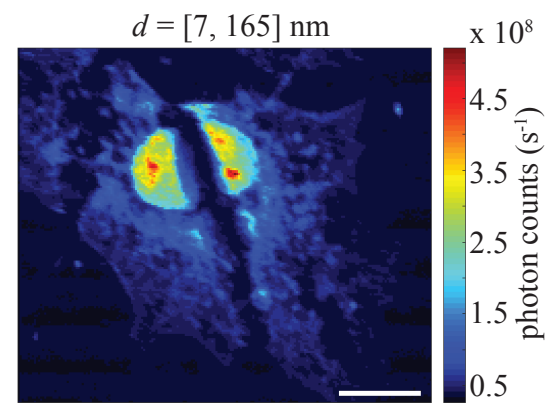

c)

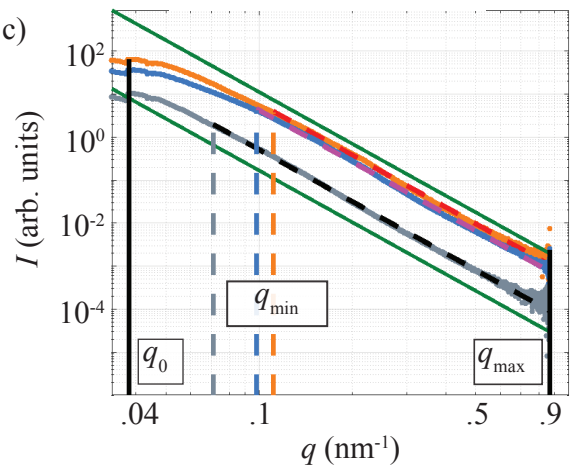

b)

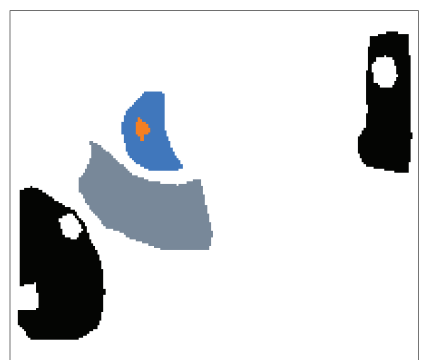

d)

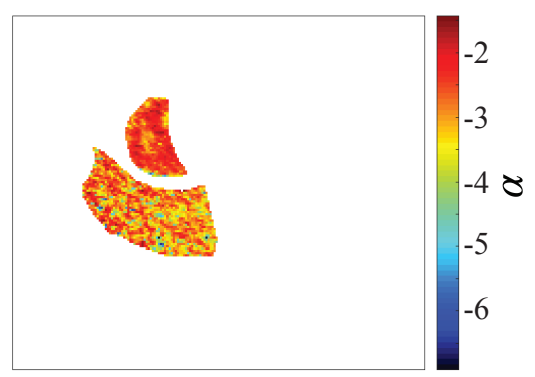

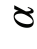
. e)

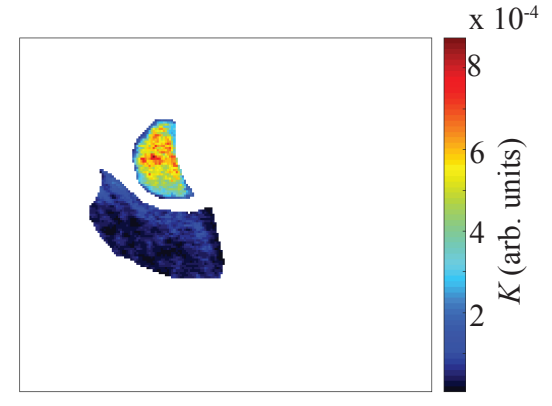

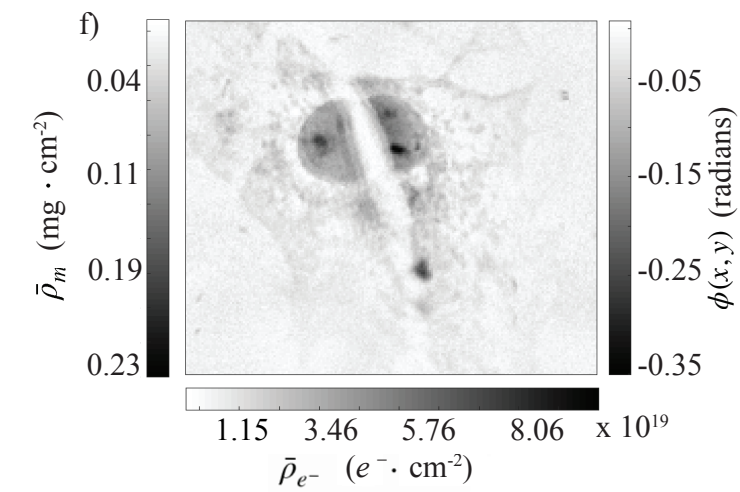

Figure B.27: Sample AW-PD06-C36. The scale bar in a) is $10 \mu \mathrm{m}$ and applies to subfigures b), d), e) and f). See page 117.

\begin{tabular}{|c|c|c|c|c|c|c|}
\hline Sample & ROI & $\alpha$ & $K$ (arb.units) & $-\phi(\mathrm{rad})$ & $\bar{\rho}_{m}\left(\frac{\mathrm{mg}}{\mathrm{cm}^{2}}\right)$ & $\bar{\rho}_{e^{-}}\left(\frac{\mathrm{e}^{-}}{\mathrm{cm}^{2}}\right)$ \\
\hline \multirow{3}{*}{ AW-PD06-C36 } & Heterochromatin & -2.84 & $6.07 \times 10^{-4}$ & 0.20 & 0.16 & $4.69 \times 10^{19}$ \\
& Euchromatin & -2.51 & $4.22 \times 10^{-4}$ & 0.12 & 0.09 & $2.78 \times 10^{19}$ \\
& Cytoplasm & -3.02 & $5.12 \times 10^{-5}$ & 0.03 & 0.03 & $8.01 \times 10^{18}$ \\
\hline
\end{tabular}


a)

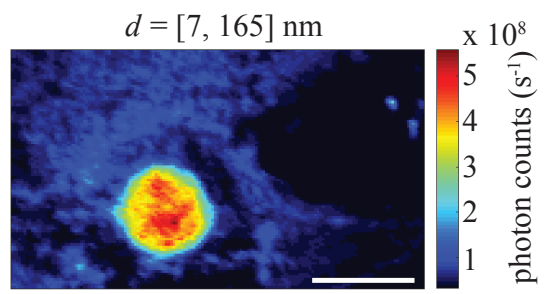

c)

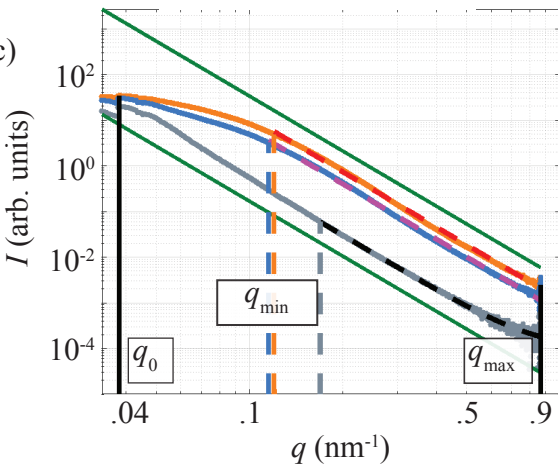

e)

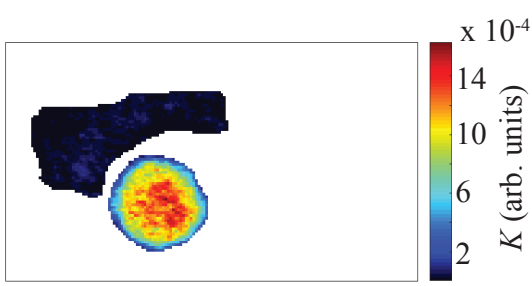

b)

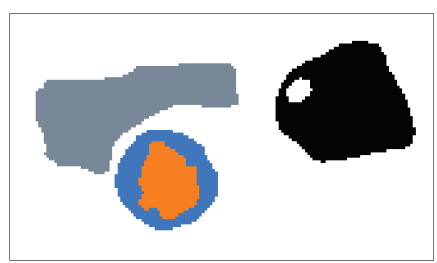

d)

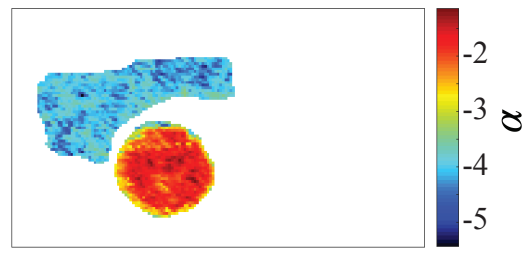

f)

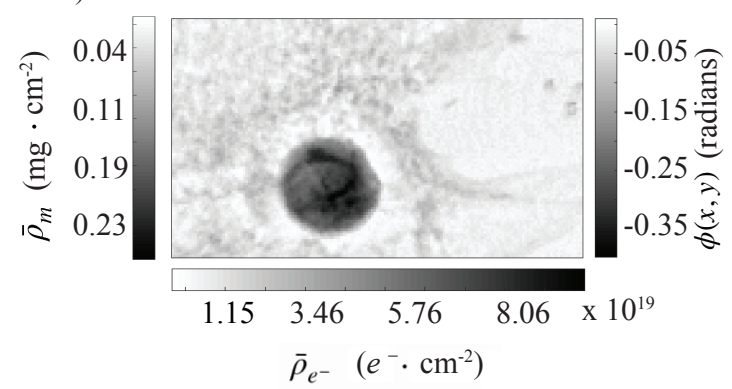

Figure B.28: Sample AW-PD06-C38. The scale bar in a) is $10 \mu \mathrm{m}$ and applies to subfigures b), d), e) and f). See page 117 .

\begin{tabular}{|c|c|c|c|c|c|c|}
\hline Sample & ROI & $\alpha$ & $K$ (arb.units) & $-\phi(\mathrm{rad})$ & $\bar{\rho}_{m}\left(\frac{\mathrm{mg}}{\mathrm{cm}^{2}}\right)$ & $\bar{\rho}_{e^{-}}\left(\frac{\mathrm{e}^{-}}{\mathrm{cm}^{2}}\right)$ \\
\hline \multirow{3}{*}{ AW-PD06-C38 } & Heterochromatin & -1.77 & $1.18 \times 10^{-3}$ & 0.29 & 0.22 & $6.74 \times 10^{19}$ \\
& Euchromatin & -2.25 & $6.39 \times 10^{-4}$ & 0.20 & 0.15 & $4.64 \times 10^{19}$ \\
& Cytoplasm & -4.05 & $2.82 \times 10^{-5}$ & 0.05 & 0.04 & $1.18 \times 10^{19}$ \\
\hline
\end{tabular}


a)

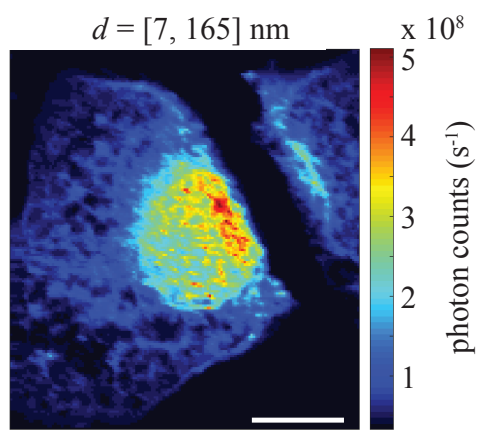

c)

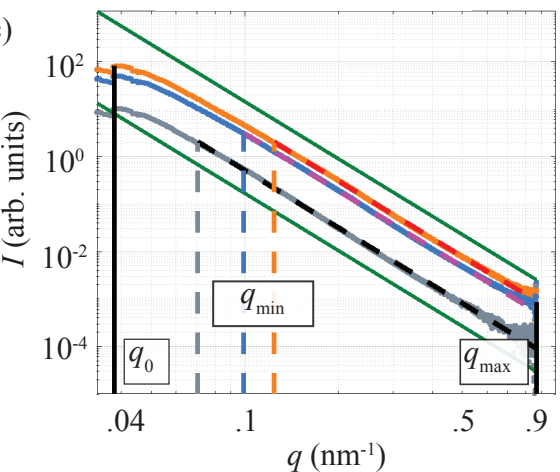

b)

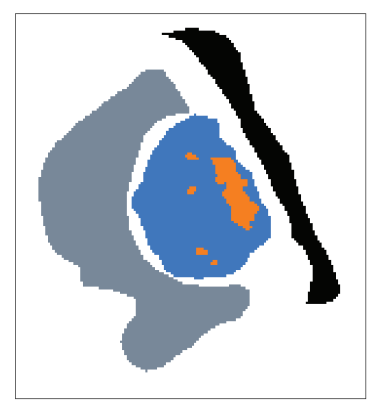

d)
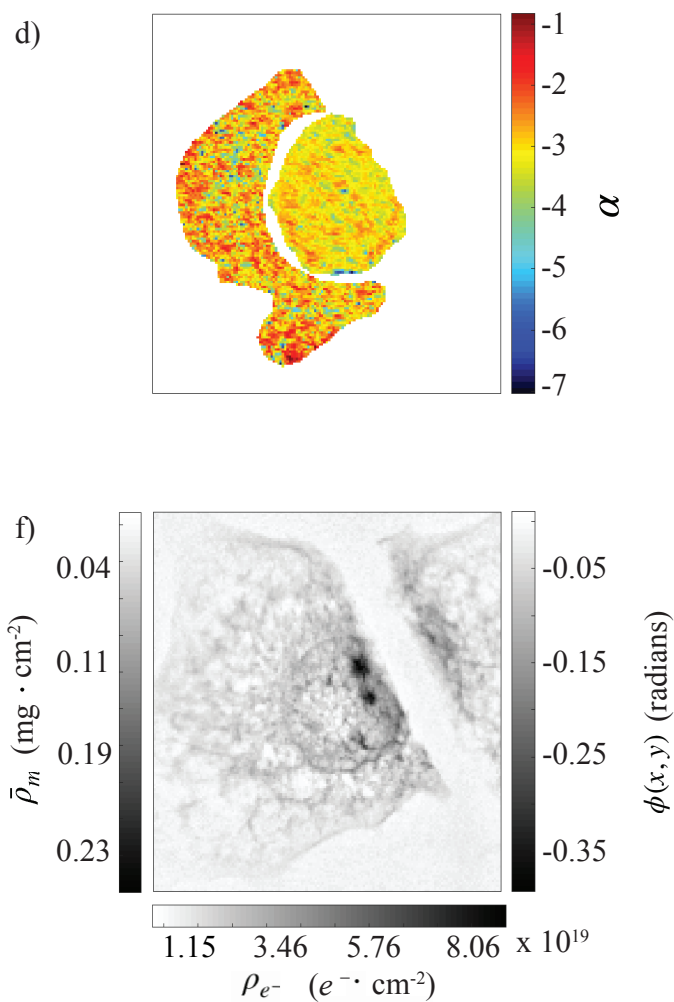

Figure B.29: Sample AW-PD06-C40. The scale bar in a) is $10 \mu \mathrm{m}$ and applies to subfigures b), d), e) and f). See page 117.

\begin{tabular}{|c|c|c|c|c|c|c|}
\hline Sample & ROI & $\alpha$ & $K$ (arb.units) & $-\phi(\mathrm{rad})$ & $\bar{\rho}_{m}\left(\frac{\mathrm{mg}}{\mathrm{cm}^{2}}\right)$ & $\bar{\rho}_{e^{-}}\left(\frac{\mathrm{e}^{-}}{\mathrm{cm}^{2}}\right)$ \\
\hline \multirow{3}{*}{ AW-PD06-C40 } & Heterochromatin & -3.30 & $4.78 \times 10^{-4}$ & 0.19 & 0.14 & $4.30 \times 10^{19}$ \\
& Euchromatin & -3.10 & $2.96 \times 10^{-4}$ & 0.08 & 0.06 & $1.93 \times 10^{19}$ \\
& Cytoplasm & -2.90 & $5.03 \times 10^{-5}$ & 0.04 & 0.03 & $8.80 \times 10^{18}$ \\
\hline
\end{tabular}


a)

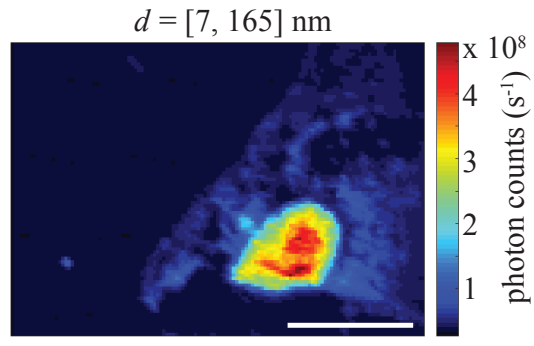

c)

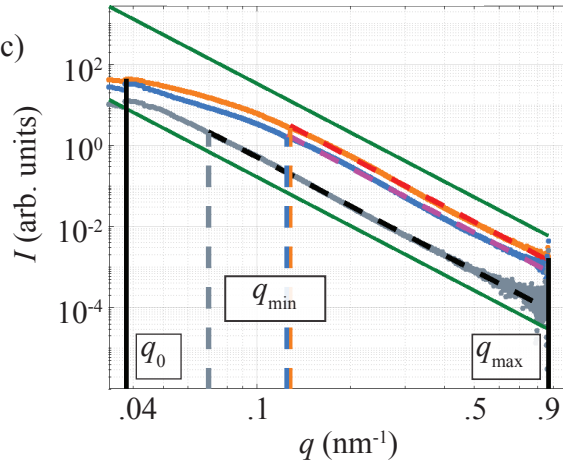

b)

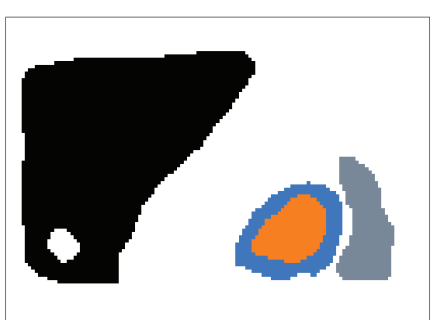

d)
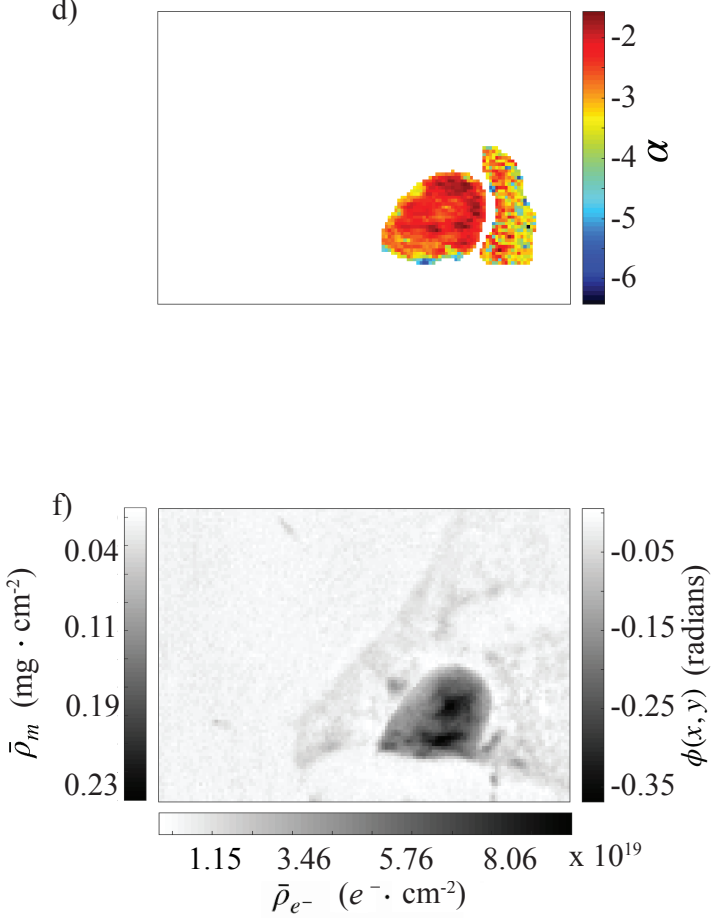

Figure B.30: Sample AW-PD06-C41. The scale bar in a) is $10 \mu \mathrm{m}$ and applies to subfigures b), d), e) and f). See page 117 .

\begin{tabular}{|c|c|c|c|c|c|c|}
\hline Sample & ROI & $\alpha$ & $K$ (arb.units) & $-\phi(\mathrm{rad})$ & $\bar{\rho}_{m}\left(\frac{\mathrm{mg}}{\mathrm{cm}^{2}}\right)$ & $\bar{\rho}_{e^{-}}\left(\frac{\mathrm{e}^{-}}{\mathrm{cm}^{2}}\right)$ \\
\hline \multirow{3}{*}{ AW-PD06-C41 } & Heterochromatin & -2.31 & $8.23 \times 10^{-4}$ & 0.24 & 0.19 & $5.58 \times 10^{19}$ \\
& Euchromatin & -2.73 & $4.41 \times 10^{-4}$ & 0.16 & 0.12 & $3.74 \times 10^{19}$ \\
& Cytoplasm & -3.25 & $5.03 \times 10^{-5}$ & 0.04 & 0.03 & $9.36 \times 10^{18}$ \\
\hline
\end{tabular}


a)

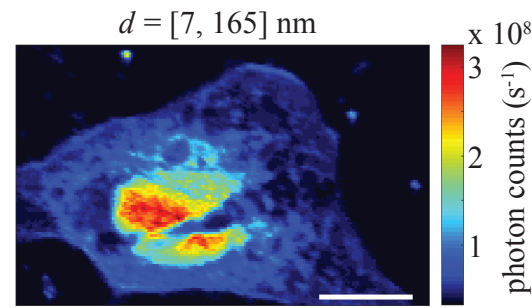

c)

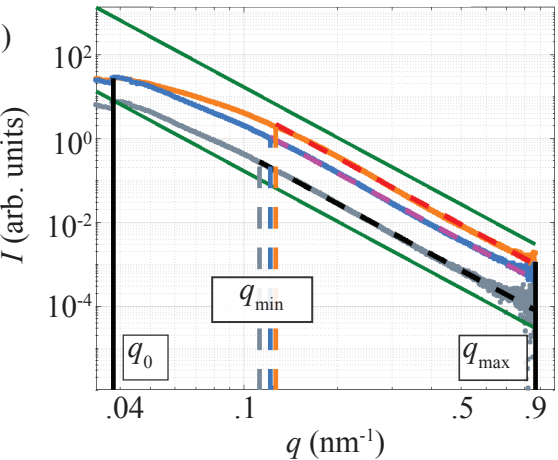

e)

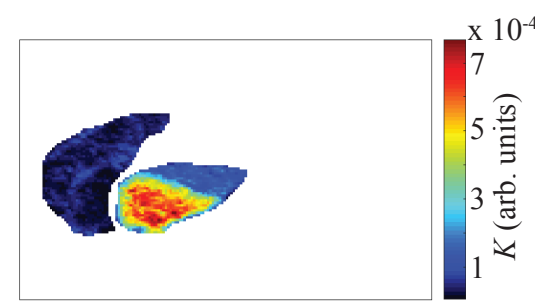

b)

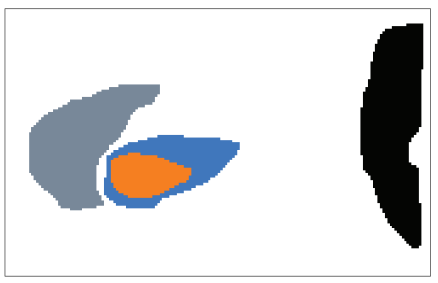

d)
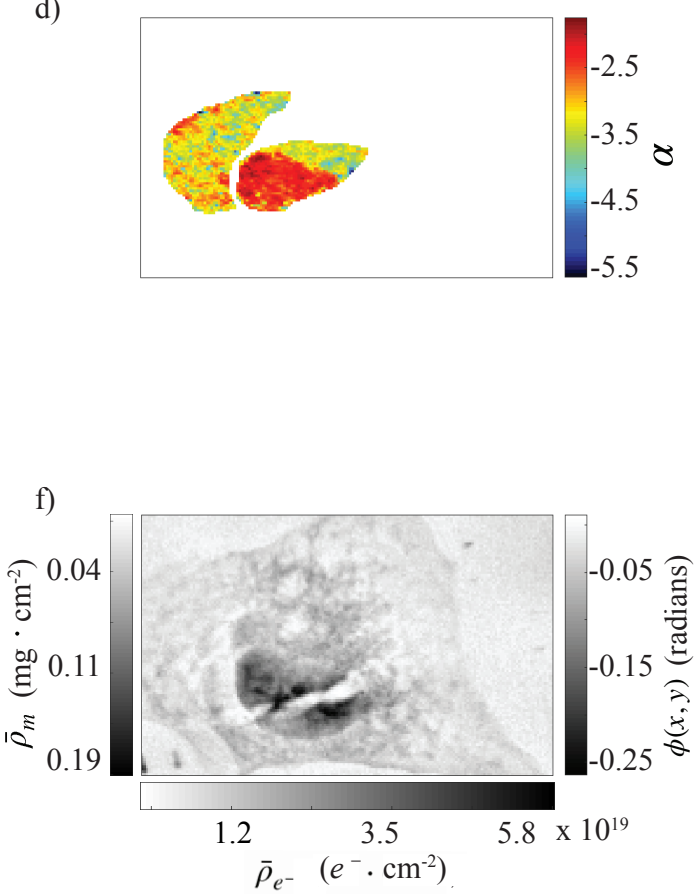

Figure B.31: Sample AW-PD06-C42. The scale bar in a) is $10 \mu \mathrm{m}$ and applies to subfigures b), d), e) and f). See page 117 .

\begin{tabular}{|c|c|c|c|c|c|c|}
\hline Sample & ROI & $\alpha$ & $K$ (arb.units) & $-\phi(\mathrm{rad})$ & $\bar{\rho}_{m}\left(\frac{\mathrm{mg}}{\mathrm{cm}^{2}}\right)$ & $\bar{\rho}_{e^{-}}\left(\frac{\mathrm{e}^{-}}{\mathrm{cm}^{2}}\right)$ \\
\hline \multirow{3}{*}{ AW-PD06-C42 } & Heterochromatin & -2.32 & $5.57 \times 10^{-4}$ & 0.14 & 0.10 & $3.14 \times 10^{19}$ \\
& Euchromatin & -3.05 & $2.27 \times 10^{-4}$ & 0.09 & 0.07 & $2.12 \times 10^{19}$ \\
& Cytoplasm & -3.23 & $3.76 \times 10^{-5}$ & 0.03 & 0.02 & $6.11 \times 10^{18}$ \\
\hline
\end{tabular}


a)
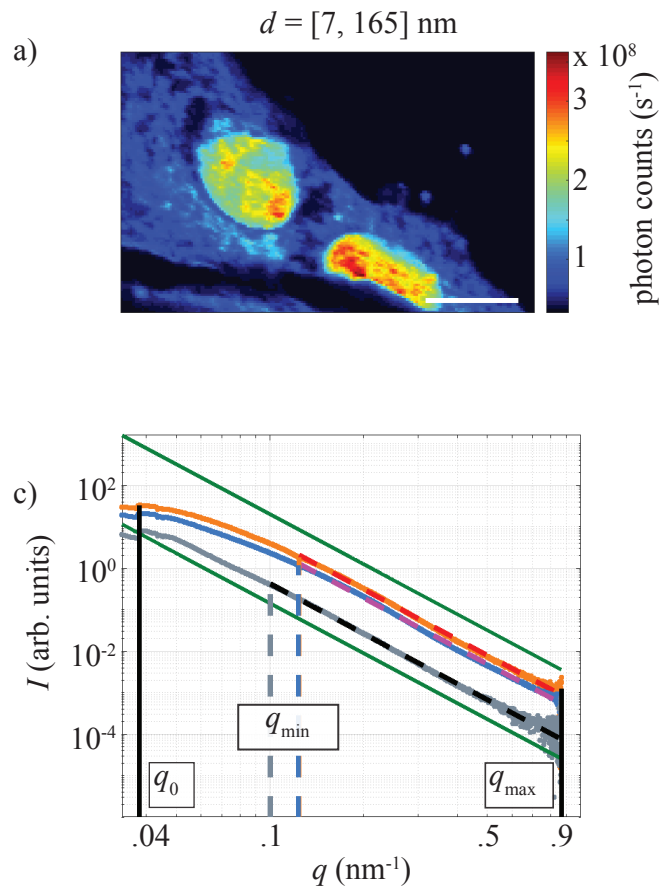

e)

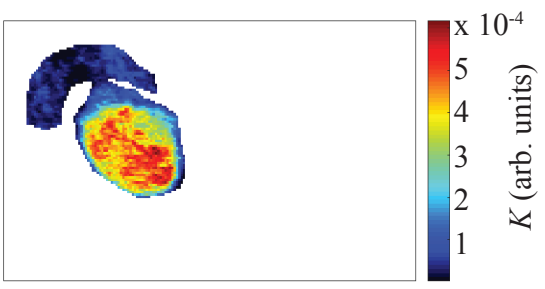

b)

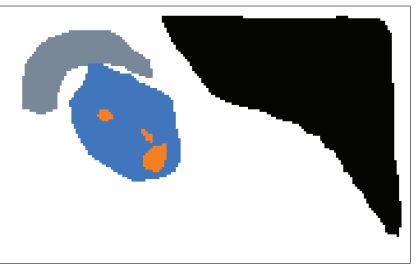

d)
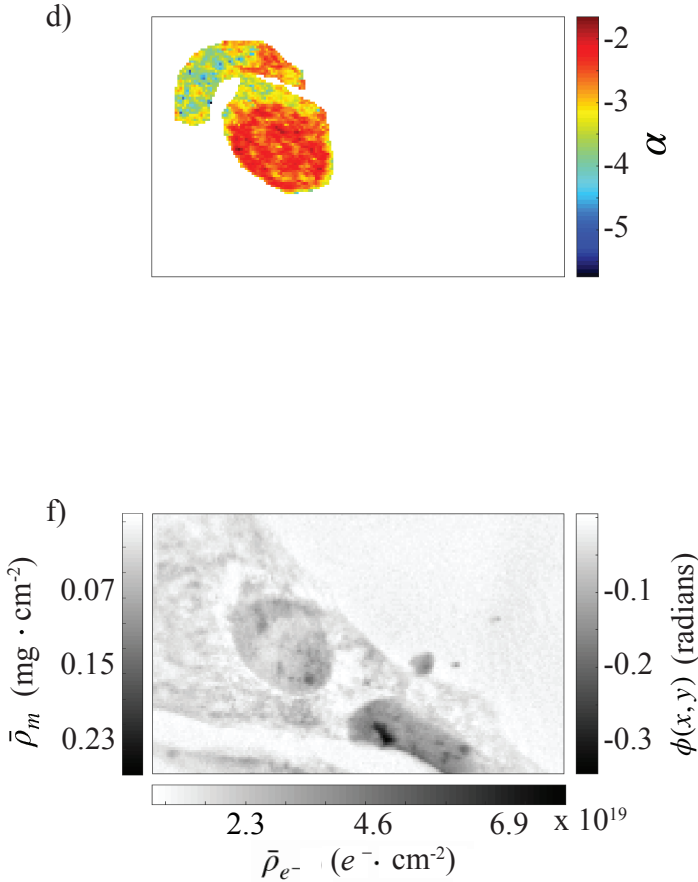

Figure B.32: Sample AW-PD06-C43A. The scale bar in a) is $10 \mu \mathrm{m}$ and applies to subfigures b), d), e) and f). See page 117 .

\begin{tabular}{|c|c|c|c|c|c|c|}
\hline Sample & ROI & $\alpha$ & $K$ (arb.units) & $-\phi(\mathrm{rad})$ & $\bar{\rho}_{m}\left(\frac{\mathrm{mg}}{\mathrm{cm}}\right)$ & $\bar{\rho}_{e^{-}}\left(\frac{\mathrm{e}^{-}}{\mathrm{cm}^{2}}\right)$ \\
\hline \multirow{3}{*}{ AW-PD06-C43A } & Heterochromatin & -2.53 & $4.99 \times 10^{-4}$ & 0.11 & 0.08 & $2.50 \times 10^{19}$ \\
& Euchromatin & -2.68 & $3.07 \times 10^{-4}$ & 0.06 & 0.05 & $1.45 \times 10^{19}$ \\
& Cytoplasm & -3.32 & $3.75 \times 10^{-5}$ & 0.03 & 0.03 & $7.85 \times 10^{18}$ \\
\hline
\end{tabular}


a)

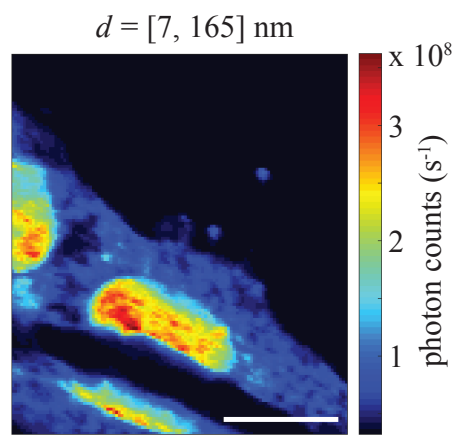

c)

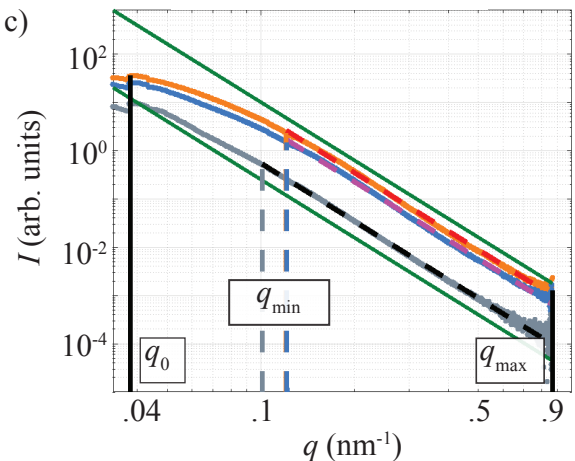

e)

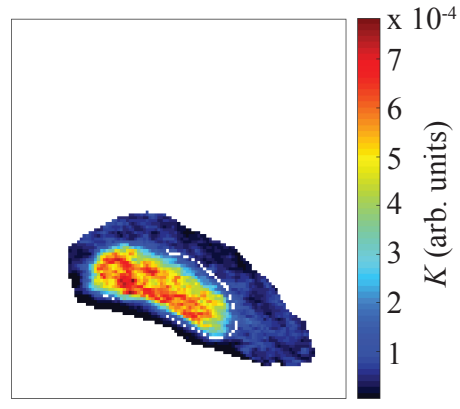

b)

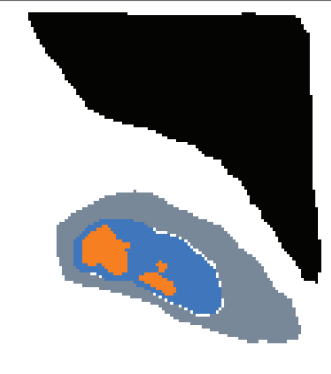

d)
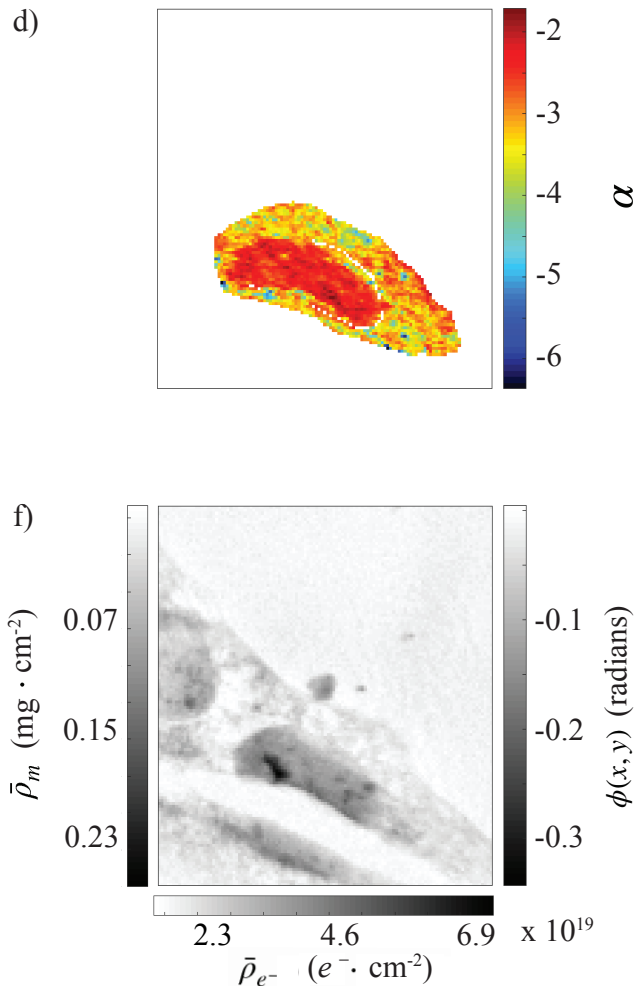

Figure B.33: Sample AW-PD06-C43B. The scale bar in a) is $10 \mu \mathrm{m}$ and applies to subfigures b), d), e) and f). See page 117.

\begin{tabular}{|c|c|c|c|c|c|c|}
\hline Sample & ROI & $\alpha$ & $K$ (arb.units) & $-\phi(\mathrm{rad})$ & $\bar{\rho}_{m}\left(\frac{\mathrm{mg}}{\mathrm{cm}^{2}}\right)$ & $\bar{\rho}_{e^{-}}\left(\frac{\mathrm{e}^{-}}{\mathrm{cm}^{2}}\right)$ \\
\hline \multirow{3}{*}{ AW-PD06-C43B } & Heterochromatin & -2.46 & $5.60 \times 10^{-4}$ & 0.17 & 0.13 & $4.00 \times 10^{19}$ \\
& Euchromatin & -2.65 & $3.60 \times 10^{-4}$ & 0.11 & 0.08 & $2.51 \times 10^{19}$ \\
& Cytoplasm & -3.27 & $5.03 \times 10^{-5}$ & 0.03 & 0.02 & $6.80 \times 10^{18}$ \\
\hline
\end{tabular}




\section{References}

1. Maeshima, K., Imai, R., Tamura, S. \& Nozaki, T. Chromatin as Dynamic 10-nm Fibers. Chromosoma 123, 225-237 (2014).

2. Hodges, A. et al. Regional and Cellular Gene Expression Changes in Human Huntington's Disease Brain. Hum. Mol. Genet. 15, 965-977 (2006).

3. Ralston, S. Genetic Control of Susceptibility to Osteoporosis. J. Clin. Endocrinol. Metab. 87, 2460-2466 (2002).

4. Rees, D., Williams, T. \& Gladwin, M. Sickle-Cell Disease. Lancet 376, 2018-2031 (2010).

5. Finch, J. \& Klug, A. Solenoidal Model for Superstructure in Chromatin. Proc. Natl. Acad. Sci. U.S.A. 73, 1897-1901 (1976).

6. Sedat, J. \& Manuelidis, L. A Direct Approach to the Structure of Eukaryotic Chromosomes in Cold Spring Harbor Symposia on Quantitative Biology 42 (1978), $331-350$.

7. Olins, D. \& Olins, A. Chromatin History: Our View from the Bridge. Nat. Rev. Mol. Cell Biol. 4, 809-814 (2003).

8. Woodcock, C., Frado, L. \& Rattner, J. The Higher-Order Structure of Chromatin: Evidence for a Helical Ribbon Arrangement. J. Cell Biol. 99, 42-52 (1984).

9. Maeshima, K., Hihara, S. \& Eltsov, M. Chromatin Structure: Does the 30-nm Fiber Exist in vivo? Curr. Opin. Cell Biol. 22, 291-297 (2010).

10. Maeshima, K., Ide, S. \& Babokhov, M. Dynamic Chromatin Organization without the 30-nm Fiber. Curr. Opin. Cell Biol. 58, 95-104 (2019).

11. Schwartz, U., Németh, A., Diermeier, S., Exler, J., Hansch, S., Maldonado, R., Heizinger, L., Merkl, R. \& Längst, G. Characterizing the Nuclease Accessibility of DNA in Human Cells to Map Higher Order Structures of Chromatin. Nucleic Acids Res. 47, 1239-1254 (2019). 
12. Franklin, R. \& Gosling, R. Molecular Configuration in Sodium Thymonucleate. Nature 171, 740-741 (1953).

13. Wilkins, M., Stokes, A. \& Wilson, H. Molecular Structure of Nucleic Acids: Molecular Structure of Deoxypentose Nucleic Acids. Nature 171, 738-740 (1953).

14. Watson, J. \& Crick, F. Molecular Structure of Nucleic Acids. Nature 171, 737738 (1953).

15. Gabor, D. A New Microscopic Principle. Nature 161, 777-778 (1948).

16. Miescher-Rüsch, F. Über die chemische Zusammensetzung der Eiterzellen. Medicinischchemische Untersuchungen 4, 441-460 (1871).

17. Flemming, W. Zellsubstanz, Kern und Zelltheilung (FCW Vogel, 1882).

18. Kossel, A. Über die chemische Beschaffenheit des Zellkerns. München Med. Wochenschrift 58, 65-69 (1911).

19. Avery, O., MacLeod, C. \& McCarty, M. Studies on the Chemical Nature of the Substance Inducing Transformation of Pneumococcal Types: Induction of Transformation by a Desoxyribonucleic Acid Fraction Isolated from Pneumococcus Type III. J. Exp. Med. 79, 137-158 (1944).

20. Griffith, F. The Significance of Pneumococcal Types. Int. J. Hyg. Environ. Health 27, 113-159 (1928).

21. Pauling, L., Corey, R. B. \& Branson, H. R. The Structure of Proteins: Two Hydrogen-Bonded Helical Configurations of the Polypeptide Chain. Proc. Natl. Acad. Sci. U.S.A. 37, 205-211 (1951).

22. Pauling, L. The Nature of the Chemical Bond. J. Am. Chem. Soc. 53, 1367-1400 (1931).

23. Luzzati, V. \& Nicolaieff, A. The Structure of Nucleohistones and Nucleoprotamines. J. Mol. Biol. 7, 142-163 (1963).

24. Pardon, J., Wilkins, M. \& Richards, B. Super-Helical Model for Nucleohistone. Nature 215, 508-509 (1967).

25. Bram, S. The Secondary Structure of DNA in Solution and in Nucleohistone. $J$. Mol. Biol. 58, 277-288 (1971).

26. Everid, A., Small, J. \& Davies, H. Electron-Microscope Observations on the Structure of Condensed Chromatin: Evidence for Orderly Arrays of Unit Threads on the Surface of Chicken Erythrocyte Nuclei. J. Cell Sci. 7, 35-48 (1970).

27. Woodcock, C. Ultrastructure of Inactive Chromatin in J. Cell. Biol. 59 (1973), A368. 
28. Olins, A. \& Olins, D. Spheroid Chromatin Units ( $\nu$ bodies). Science 183, 330-332 (1974).

29. Woodcock, C., Safer, J. \& Stanchfield, J. Structural Repeating Units in Chromatin: I. Evidence for Their General Occurrence. Exp. Cell Res. 97, 101-110 (1976).

30. Richmond, T., Finch, J., Rushton, B., Rhodes, D. \& Klug, A. Structure of the Nucleosome Core Particle at $7 \AA$ Resolution. Nature 311, 532-537 (1984).

31. Hémonnot, C., Ranke, C., Saldanha, O., Graceffa, R., Hagemann, J. \& Köster, S. Following DNA Compaction During the Cell Cycle by X-ray Nanodiffraction. ACS Nano 10, 10661-10670 (2016).

32. Jiang, H., Song, C., Chen, C., Xu, R., Raines, K., Fahimian, B., Lu, C., Lee, T., Nakashima, A. \& Urano, J. e. Quantitative 3D Imaging of Whole, Unstained Cells by Using X-ray Diffraction Microscopy. Proc. Natl. Acad. Sci. U.S.A. 107, 11234-11239 (2010).

33. Giewekemeyer, K., Thibault, P., Kalbfleisch, S., Beerlink, A., Kewish, C., Dierolf, M., Pfeiffer, F. \& Salditt, T. Quantitative Biological Imaging by Ptychographic X-ray Diffraction Microscopy. Proc. Natl. Acad. Sci. U.S.A. 107, 529-534 (2010).

34. Bartels, M., Krenkel, M., Haber, J., Wilke, R. \& Salditt, T. X-ray Holographic Imaging of Hydrated Biological Cells in Solution. Phys. Rev. Lett. 114, 048103 (2015).

35. Bartels, M., Priebe, M., Wilke, R., Krüger, S., Giewekemeyer, K., Kalbfleisch, S., Olendrowitz, C., Sprung, M. \& Salditt, T. Low-Dose Three-Dimensional Hard X-ray Imaging of Bacterial Cells. Opt. Nanoscopy 1, 1-7 (2012).

36. Cloetens, P., Ludwig, W., Baruchel, J., Van Dyck, D., Van Landuyt, J., Guigay, J. \& Schlenker, M. Holotomography: Quantitative Phase Tomography with Micrometer Resolution using Hard Synchrotron Radiation X-rays. Appl. Phys. Lett. 75, 2912-2914 (1999).

37. Wilke, R., Priebe, M., Bartels, M., Giewekemeyer, K., Diaz, A., Karvinen, P. \& Salditt, T. Hard X-ray Imaging of Bacterial Cells: Nanodiffraction and Ptychographic Reconstruction. Opt. Express 20, 19232-19254 (2012).

38. Diaz, A. et al. Three-Dimensional Mass Density Mapping of Cellular Ultrastructure by Ptychographic X-ray Nanotomography. J. Struct. Biol. 192, 461-469 (2015). 
39. Song, C., Takagi, M., Park, J., Xu, R., Gallagher-Jones, M., Imamoto, N. \& Ishikawa, T. Analytic 3D Imaging of Mammalian Nucleus at Nanoscale Using Coherent X-rays and Optical Fluorescence Microscopy. Biophys. J. 107, 10741081 (2014).

40. Spahn, C., Grimm, J., Lavis, L., Lampe, M. \& Heilemann, M. Whole-Cell, 3D, and Multicolor STED Imaging with Exchangeable fluorophores. Nano Lett. 19, 500-505 (2018).

41. Marsden, M. \& Laemmli, U. Metaphase Chromosome Structure: Evidence for a Radial Loop Model. Cell 17, 849-858 (1979).

42. Ou, H., Phan, S., Deerinck, T., Thor, A., Ellisman, M. \& O'shea, C. ChromEMT: Visualizing 3D Chromatin Structure and Compaction in Interphase and Mitotic Cells. Science 357 (2017).

43. Li, Y., Roth, E., Agrawal, V., Eshein, A., Fredrick, J., Almassalha, L., Shim, A., Bleher, R., Dravid, V. \& Backman, V. Quantifying Three-Dimensional Chromatin Organization Utilizing Scanning Transmission Electron Microscopy: ChromSTEM. bioRxiv (2019).

44. Singer, C. Notes on the Early History of Microscopy. J. R. Soc. Med. 7, 247-279 (1914).

45. Abbe, E. Beiträge zur Theorie des Mikroskops und der mikroskopischen Wahrnehmung. Archiv für mikroskopische Anatomie 9, 413-418 (1873).

46. Born, M. \& Wolf, E. Principles of Optics, 7th edition (Press Syndicate of the University of Cambridge, 1999).

47. Rayleigh, J. XV. On the Theory of Optical Images, with Special Reference to the Microscope. Philosophical Magazine Series 5 42, 167-195 (1896).

48. Lakowicz, J. Principles of Fluorescence Spectroscopy (Kluwer Academic Plenum Press, New York, 1999).

49. So, P., Dong, C., Masters, B. \& Berland, K. Two-Photon Excitation Fluorescence Microscopy. Annu. Rev. Biomed. Eng. 2, 399-429 (2000).

50. Jablonski, A. Efficiency of Anti-Stokes Fluorescence in Dyes. Nature 131, 839-840 (1933).

51. Stokes, G. On the Change of Refrangibility of Light. Philos. Trans. Royal Soc. 142, 463-562 (1852).

52. Lichtman, J. \& Conchello, J. Fluorescence Microscopy. Nat. Methods 2, 910-919 (2005).

53. Jonkman, J. \& Stelzer, E. Resolution and Contrast in Confocal and Two-Photon Microscopy 101-125 (New York: Wiley-Liss, 2002). 
54. Darzynkiewicz, Z., Robinson, J. \& Roederer, M. Essential Cytometry Methods (Academic Press, 2009).

55. Hell, S. \& Wichmann, J. Breaking the Diffraction Resolution Limit by Stimulated Emission: Stimulated-Emission-Depletion Fluorescence Microscopy. Opt. Lett. 19, 780-782 (1994).

56. Klar, T. \& Hell, S. Subdiffraction Resolution in Far-Field Fluorescence Microscopy. Opt. Lett. 24, 954-956 (1999).

57. Göttfert, F., Wurm, C., Mueller, V., Berning, S., Cordes, V., Honigmann, A. \& Hell, S. Coaligned Dual-Channel STED Nanoscopy and Molecular Diffusion Analysis at $20 \mathrm{~nm}$ Resolution. Biophys. J. 105, L01-L03 (2013).

58. Westphal, V. \& Hell, S. Nanoscale Resolution in the Focal Plane of an Optical Microscope. Phys. Rev. Lett. 94, 143903 (2005).

59. Rittweger, E., Han, K., Irvine, S., Eggeling, C. \& Hell, S. STED Microscopy Reveals Crystal Colour Centres with Nanometric Resolution. Nat. Photonics. 3, 144 (2009).

60. Mitchell-Jordan, S., Chen, H., Franklin, S., Stefani, E., Bentolila, L. \& Vondriska, T. Features of Endogenous Cardiomyocyte Chromatin Revealed by SuperResolution STED Microscopy. J. Mol. Cell. Cardiol. 53, 552-558 (2012).

61. Monte, E. et al. Reciprocal Regulation of the Cardiac Epigenome by Chromatin Structural Proteins Hmgb and Ctcf Implications for Transciptional Regulation. J. Biol. Chem. 291, 15428-15446 (2016).

62. Hell, S. Far-Field Optical Nanoscopy. Science 316, 1153-1158 (2007).

63. Dyba, M. \& Hell, S. Focal Spots of Size $\lambda / 23$ Open Up Far-Field Florescence Microscopy at $33 \mathrm{~nm}$ Axial Resolution. Phys. Rev. Lett. 88, 163901 (2002).

64. Harke, B., Keller, J., Ullal, C., Westphal, V., Schönle, A. \& Hell, S. Resolution Scaling in STED Microscopy. Opt. Express 16, 4154-4162 (2008).

65. Dyba, M., Keller, J. \& Hell, S. Phase Filter Enhanced STED-4Pi Fluorescence Microscopy: Theory and Experiment. New J. Phys. 7, 134 (2005).

66. Balzarotti, F., Eilers, Y., Gwosch, K., Gynnå, A., Westphal, V., Stefani, F., Elf, J. \& Hell, S. Nanometer Resolution Imaging and Tracking of Fluorescent Molecules with Minimal Photon Fluxes. Science 355, 606-612 (2017).

67. Röntgen, W. On a New Kind of Rays. Science 3, 227-231 (1896).

68. Salditt, T., Aspelmeier, T. \& Aeffner, S. Biomedical Imaging: Principles of Radiography, Tomography and Medical Physics (Walter de Gruyter GmbH \& Co KG, 2017). 
69. Guinier, A., Fournet, G. \& Yudowitch, K. Small-Angle Scattering of X-rays (Wiley New York, 1955).

70. Glatter, O. \& Kratky, O. Small-Angle X-ray Scattering (Academic Press, 1982).

71. Als-Nielsen, J. \& McMorrow, D. Elements of Modern X-ray Physics (John Wiley \& Sons, 2011).

72. Stangl, J., Mocuta, C., Chamard, V. \& Carbone, D. Nanobeam X-ray Scattering: Probing Matter at the Nanoscale (John Wiley \& Sons, 2013).

73. Berger, M. \& Hubbell, J. XCOM: Photon Cross Sections on a Personal Computer tech. rep. (National Bureau of Standards, 1987).

74. Howells, M. R., Beetz, T., Chapman, H. N., Cui, C., Holton, J., Jacobsen, C., Kirz, J., Lima, E., Marchesini, S. \& Miao, H. An Assessment of the Resolution Limitation Due to Radiation Damage in X-ray Diffraction Microscopy. J. Electron Spectrosc. Relat. Phenom. 170, 4-12 (2009).

75. Hertz, H. Über einen Einfluss des ultravioletten Lichtes auf die Electrische Entladung. Ann. Phys. 267, 983-1000 (1887).

76. Arons, A. \& Peppard, M. Einstein's Proposal of the Photon Concept-a Translation of the Annalen der Physik Paper of 1905. Am. J. Phys. 33, 367-374 (1965).

77. Compton, A. The Spectrum of Scattered X-rays. Phys. Rev. 22, 409 (1923).

78. Thomson, J. Conduction of Electricity Through Gases (Watchmaker Publishing, 2005).

79. Rayleigh, J. On the Scattering of Light by Small Particles (1871).

80. Bragg, W. \& Bragg, L. The Reflection of X-rays by Crystals. Proc. R. Soc. A. 88, 428-438 (1913).

81. Pedersen, J. Analysis of Small-Angle Scattering Data from Colloids and Polymer Solutions: Modeling and Least-Squares Fitting. Adv. Colloid Interface Sci. 70, 171-210 (1997).

82. Todaro, G. J. \& Green, H. Quantitative Studies of the Growth of Mouse Embryo Cells in Culture and Their Development into Established Lines. J. Cell Biol. 17, 299-313 (1963).

83. Kolb, H., Finn, M. \& Sharpless, K. Click Chemistry: Diverse Chemical Function from a Few Good Reactions. Angew. Chem. Int. 40, 2004-2021 (2001).

84. Porod, G. Die Röntgenkleinwinkelstreuung von dichtgepackten colloiden Systemen. Kolloid-Zeitschrift 124, 83-114 (1951). 
85. Debye, P., Anderson Jr, H. \& Brumberger, H. Scattering by an Inhomogeneous Solid. II. The Correlation Function and its Application. J. Appl. Phys. 28, 679683 (1957).

86. Ruland, W. Small-Angle Scattering of Two-Phase Systems: Determination and Significance of Systematic Deviations from Porod's Law. J. Appl. Crystallogr. 4, 70-73 (1971).

87. Mahendrasingam, A., Martin, C., Fuller, W., Blundell, D., MacKerron, D., Rule, R., Oldman, R., Liggat, J., Riekel, C. \& Engström, P. Microfocus X-ray Diffraction of Spherulites of Poly-3-hydroxybutyrate. J. Synchrotron Radiat. 2, 308-312 (1995).

88. Fratzl, P., Jakob, H., Rinnerthaler, S., Roschger, P. \& Klaushofer, K. PositionResolved Small-Angle X-ray Scattering of Complex Biological Materials. J. Appl. Crystallogr. 30, 765-769 (1997).

89. Deyhle, H., Bunk, O. \& Müller, B. Nanostructure of Healthy and Caries-Affected Human Teeth. Nanomedicine: NBM 7, 694-701 (2011).

90. Stanić, V., Bettini, J., Montoro, F., Stein, A. \& Evans-Lutterodt, K. Local Structure of Human Hair Spatially Resolved by Sub-Micron X-ray Beam. Sci. Rep. 5, 17347 (2015).

91. Weinhausen, B., Nolting, J., Olendrowitz, C., Langfahl-Klabes, J., Reynolds, M., Salditt, T. \& Köster, S. X-ray Nano-Diffraction on Cytoskeletal Networks. New J. Phys. 14, 085013 (2012).

92. Weinhausen, B., Saldanha, O., Wilke, R. N., Dammann, C., Priebe, M., Burghammer, M., Sprung, M. \& Köster, S. Scanning X-ray Nanodiffraction on Living Eukaryotic Cells in Microfluidic Environments. Phys. Rev. Lett. 112, 088102 (2014).

93. Bernhardt, M., Nicolas, J.-D., Osterhoff, M., Mittelstädt, H., Reuss, M., Harke, B., Wittmeier, A., Sprung, M., Köster, S. \& Salditt, T. Correlative Microscopy Approach for Biology using X-ray Holography, X-ray Scanning Diffraction and STED Microscopy. Nat. Commun. 9, 3641 (2018).

94. Cassini, C., Wittmeier, A., Brehm, G., Denz, M., Burghammer, M. \& Köster, S. Large Field-of-View Scanning Small-Angle X-ray Scattering of Mammalian Cells. J. Synchrotron Radiat. 27 (2020).

95. Kalbfleisch, S., Neubauer, H., Krüger, S., Bartels, M., Osterhoff, M., Mai, D., Giewekemeyer, K., Hartmann, B., Sprung, M. \& Salditt, T. The Göttingen Holography Endstation of Beamline P10 at PETRA III/DESY. AIP Conf. Proc. 1365, 96-99 (2011). 
96. Salditt, T., Osterhoff, M., Krenkel, M., Wilke, R., Priebe, M., Bartels, M., Kalbfleisch, S. \& Sprung, M. Compound Focusing Mirror and X-ray Waveguide Optics for Coherent Imaging and Nanodiffraction. J. Synchrotron Radiat. 22, 867-878 (2015).

97. Salditt, T., Egner, A. \& Luke, R. Nanoscale Photonic Imaging chap. 13 (Springer Nature, 2020).

98. Kirkpatrick, P. \& Baez, A. V. Formation of Optical Images by X-rays. J. Opt. Soc. Am. 38, 766-774 (1948).

99. Zabler, S., Cloetens, P., Guigay, J., Baruchel, J. \& Schlenker, M. Optimization of Phase Contrast Imaging Using Hard X-rays. Rev. Sci. Instrum. 76, 073705 (2005).

100. Krüger, S., Neubauer, H., Bartels, M., Kalbfleisch, S., Giewekemeyer, K., Wilbrandt, P., Sprung, M. \& Salditt, T. Sub-10 nm Beam Confinement by X-ray Waveguides: Design, Fabrication and Characterization of Optical Properties. J. Synchrotron Radiat. 19, 227-236 (2012).

101. Cloetens, P. Contribution to Phase Contrast Imaging, Reconstruction and Tomography with Hard Synchrotron Radiation. PhD thesis (Vrije Universiteit Brussel, 1999).

102. Allen, L. \& Oxley, M. Phase Retrieval from Series of Images Obtained by Defocus Variation. Opt. Commun. 199, 65-75 (2001).

103. Paganin, D. Coherent X-ray Optics 6 (Oxford University Press on Demand, 2006).

104. Cloetens, P., Ludwig, W., Baruchel, J., Guigay, J., Pernot-Rejmánková, P., Salomé-Pateyron, M., Schlenker, M., Buffière, J., Maire, E. \& Peix, G. Hard X-ray Phase Imaging using Simple Propagation of a Coherent Synchrotron Radiation Beam. J. Phys. D Appl. Phys. 32, A145 (1999).

105. Luke, D. Relaxed Averaged Alternating Reflections for Diffraction Imaging. Inverse Probl. 21, 37 (2005).

106. Chen, H., Hoffmann, S. \& Salditt, T. X-ray Beam Compression by Tapered Waveguides. Appl. Phys. Lett. 106, 194105 (2015).

107. Guizar-Sicairos, M., Thurman, S. T. \& Fienup, J. R. Efficient Subpixel Image Registration Algorithms. Opt. Lett. 33, 156-158 (2008).

108. Nicolas, J.-D., Bernhardt, M., Krenkel, M., Richter, C., Luther, S. \& Salditt, T. Combined Scanning X-ray Diffraction and Holographic Imaging of Cardiomyocytes. J. Appl. Crystallogr. 50, 612-620 (2017).

109. Radon, J. On the Determination of Functions From Their Integral Values Along Certain Manifolds. IEEE Trans. Med. Imaging 5, 170-176 (1986). 
110. Kak, A. Image Reconstruction from Projections. IET Image Process 2, 111 (1984).

111. Natterer, F. The Mathematics of Computerized Tomography (SIAM, 2001).

112. Buzug, T. in Springer Handbook of Medical Technology 311-342 (Springer, 2011).

113. Bartels, M. Cone-Beam X-ray Phase Contrast Tomography of Biological Samples: Optimization of Contrast, Resolution and Field of View $\mathrm{PhD}$ thesis (University of Göttingen, 2013).

114. Dudgeon, D. Multidimensional Digital Signal Processing. Engewood Cliffs (1983).

115. Bernhardt, M., Nicolas, J., Osterhoff, M., Mittelstädt, H., Reuss, M., Harke, B., Wittmeier, A., Sprung, M., Köster, S. \& Salditt, T. A Beamline-Compatible STED Microscope for Combined Visible-Light and X-ray Studies of Biological Matter. J. Synchrotron Radiat. 26, 1144-1151 (2019).

116. Reuss, M., Engelhardt, J. \& Hell, S. Birefringent Device Converts a Standard Scanning Microscope Into a STED Microscope that Also Maps Molecular Orientation. Opt. Express 18, 1049-1058 (2010).

117. Wittmeier, A., Cassini, C., Töpperwien, M., Denz, M., Hagemann, J., Osterhoff, M., Salditt, T. \& Köster, S. Combined Scanning Small-Angle X-ray Scattering and Holography Probes Multiple Length Scales in Cell Nuclei. J. Synchrotron Radiat. 28 (2021).

118. Koster, A. \& Klumperman, J. Electron Microscopy in Cell Biology: Integrating Structure and Function. Nat. Rev. Mol. Cell Biol. 4, SS6-SS9 (2003).

119. Lučić, V., Förster, F. \& Baumeister, W. Structural Studies by Electron Tomography: From Cells to Molecules. Annu. Rev. Biochem. 74, 833-865 (2005).

120. Stelzer, E., Wacker, I. \& De Mey, J. Confocal Fluorescence Microscopy in Modern Cell Biology. Semin. Cell Biol. 2, 145-152 (1991).

121. Sahl, S., Hell, S. \& Jakobs, S. Fluorescence Nanoscopy in Cell Biology. Nat. Rev. Mol. Cell Biol. 18, 685 (2017).

122. Kirz, J., Jacobsen, C. \& Howells, M. Soft X-ray Microscopes and Their Biological Applications. Q. Rev. Biophys. 28, 33-130 (1995).

123. Hémonnot, C. \& Köster, S. Imaging of Biological Materials and Cells by X-ray Scattering and Diffraction. ACS Nano 11, 8542-8559 (2017).

124. Lakadamyali, M. \& Cosma, M. Advanced Microscopy Methods for Visualizing Chromatin Structure. FEBS Lett. 589, 3023-3030 (2015).

125. Rust, M., Bates, M. \& Zhuang, X. Sub-Diffraction-Limit Imaging by Stochastic Optical Reconstruction Microscopy (STORM). Nat. Methods. 3, 793-796 (2006). 
126. Lakowicz, J., Szmacinski, H., Nowaczyk, K., Berndt, K. \& Johnson, M. Fluorescence Lifetime Imaging. Anal. Biochem. 202, 316-330 (1992).

127. Förster, T. Zwischenmolekulare Energiewanderung und Fluoreszenz. Annal. Phys. 437, 55-75 (1948).

128. Ricci, M., Manzo, C., García-Parajo, M., Lakadamyali, M. \& Cosma, M. Chromatin Fibers are Formed by Heterogeneous Groups of Nucleosomes in vivo. Cell 160, 1145-1158 (2015).

129. Cremazy, F., Manders, E., Bastiaens, P., Kramer, G., Hager, G., Van Munster, E., Verschure, P., Gadella Jr, T. \& van Driel, R. Imaging In-Situ Protein-DNA Interactions in the Cell Nucleus Using FRET-FLIM. Exp. Cell Res. 309, 390-396 (2005).

130. Bunk, O., Bech, M., Jensen, T., Feidenhans, R., Binderup, T., Menzel, A. \& Pfeiffer, F. Multimodal X-ray Scatter Imaging. New J. Phys. 11, 123016 (2009).

131. Wilke, R., Hoppert, M., Krenkel, M., Bartels, M. \& Salditt, T. Quantitative X-ray Phase Contrast Waveguide Imaging of Bacterial Endospores. J. Appl. Crystallogr. 48, 464-476 (2015).

132. Shen, Q., Bazarov, I. \& Thibault, P. Diffractive Imaging of Nonperiodic Materials with Future Coherent X-ray Sources. J. Synchrotron Radiat. 11, 432-438 (2004).

133. Zhang, M., Oldenhof, H., Sydykov, B., Bigalk, J., Sieme, H. \& Wolkers, W. FreezeDrying of Mammalian Cells Using Trehalose: Preservation of DNA Integrity. Sci. Rep. 7, 1-10 (2017).

134. Andersen J.and Lyon, C., Fox, A., Leung, A., Lam Y.and Steen, H., Mann, M. \& Lamond, A. Directed Proteomic Analysis of the Human Nucleolus. Curr. Biol. 12, 1-11 (2002).

135. Hernandez-Verdun, D., Roussel, P., Thiry, M., Sirri, V. \& Lafontaine, D. The Nucleolus: Structure/Function Relationship in RNA Metabolism. Wiley Interdiscip. Rev. RNA. 1, 415-431 (2010).

136. Brown, D. \& Gurdon, J. Absence of Ribosomal RNA Synthesis in the Anucleolate Mutant of Xenopus Laevis. Proc. Natl. Acad. Sci. U.S.A. 51, 139 (1964).

137. Ritossa F. Spiegelman, S. Localization of DNA Complementary to Ribosomal RNA in the Nucleolus Organizer Region of Drosophila melanogaster. Proc. Natl. Acad. Sci. U.S.A. 53, 737 (1965).

138. Phillips, R., Kleese, R. \& Wang, S. The Nucleolus Organizer Region of Maize (Zea mays L.): Chromosomal Site of DNA Complementary to Ribosomal RNA. Chromosoma 36, 79-88 (1971). 
139. Dekker, J. \& van Steensel, B. in Handbook of Systems Biology 137-151 (Elsevier, 2013).

140. Tajrishi, M., Tuteja, R. \& Tuteja, N. Nucleolin: The Most Abundant Multifunctional Phosphoprotein of Nucleolus. Commun. Integr. Biol. 4, 267-275 (2011).

141. Love, R. \& Walsh, R. The Relation of Nucleolini to Nucleolar Vacuoles in the Living Cell. Exp. Cell Res. 53, 432-446 (1968).

142. Pueschel, R., Coraggio, F. \& Meister, P. From Single Genes to Entire Genomes: the Search for a Function of Nuclear Organization. Development 143, 910-923 (2016).

143. Belmont, A., Braunfeld, M., Sedat, J. \& Agard, D. Large-Scale Chromatin Structural Domains within Mitotic and Interphase Chromosomes in vivo and in vitro. Chromosoma 98, 129-143 (1989).

144. Weiler, K. \& Wakimoto, B. Heterochromatin and Gene Expression in Drosophila. Annu. Rev. Genet. 29, 577-605 (1995).

145. Kwon S. Workman, J. The Changing Faces of HP1: From Heterochromatin Formation and Gene Silencing to Euchromatic Gene Expression: HP1 Acts as a Positive Regulator of Transcription. Bioessays 33, 280-289 (2011).

146. Ciccariello, S., Goodisman, J. \& Brumberger, H. On the Porod Law. J. Appl. Crystallogr. 21, 117-128 (1988).

147. Kolmogorov, A. N. Sulla Determinazione Empirica di una Legge di Distribuzione. Giornale dell'Istituto Italiano degli Attuari 4, 83-91 (1933).

148. Scott, D. On Optimal and Data-Based Histograms. Biometrika 66, 605-610 (1979).

149. Sadoni, N., Sullivan, K., Weinzierl, P., Stelzer, E. \& Zink, D. Large-Scale Chromatin Fibers of Living Cells Display a Discontinuous Functional Organization. Chromosoma 110, 39-51 (2001).

150. Kornberg, R. Chromatin Structure: A Repeating Unit of Histones and DNA. Science 184, 868-871 (1974).

151. Le Gros, M., Clowney, E., Magklara, A., Yen, A., Markenscoff-Papadimitriou, E., Colquitt, B., Myllys, M., Kellis, M., Lomvardas, S. \& Larabell, C. Soft X-ray Tomography Reveals Gradual Chromatin Compaction and Reorganization During Neurogenesis in vivo. Cell Rep. 17, 2125-2136 (2016).

152. Deng, J. et al. Correlative 3D X-ray Fluorescence and Ptychographic Tomography of Frozen-Hydrated Green Algae. Sci. Adv. 4, eaau4548 (2018).

153. Docampo, R. \& Moreno, S. Acidocalcisomes. Cell Calcium 50, 113-119 (2011).

154. Riley, P. Free Radicals in Biology: Oxidative Stress and the Effects of Ionizing Radiation. Int. J. Radiat. Biol. 65, 27-33 (1994). 
155. Shaw, P. \& Brown, J. Nucleoli: Composition, Function, and Dynamics. Plant Physiol. 158, 44-51 (2012).

156. Click-iT EdU Imaging Kits https://www.thermofisher.com/order/catalog/ product/C10340?ICID=search-product \#/C10340?ICID=search-product. 


\section{List of Publications}

- Andrew Wittmeier, Chiara Cassini, Mareike Töpperwien, Manuela Denz, Johannes Hagemann, Markus Osterhoff, Tim Salditt and Sarah Köster. Combined Scanning Small-Angle X-ray Scattering and Holography Probes Multiple Length Scales in Cell Nuclei. J. Synchrotron Radiat. 28 (2021)

DOI: $10.1107 /$ S1600577520016276

- Chiara Cassini, Andrew Wittmeier, Gerrit Brehm, Manuela Denz, Manfred Burghammer and Sarah Köster. Large Field-of-View Scanning Small-Angle X-ray Scattering of Mammalian Cells. J. Synchrotron Radiat. 27 (2020)

DOI: $10.1107 / \mathrm{S} 1600577520006864$

- Marten Bernhardt, Jan-David Nicolas, Markus Osterhoff, Haugen Mittelstädt, Matthias Reuss, Benjamin Harke, Andrew Wittmeier, Michael Sprung, Sarah Köster and Tim Salditt. A Beamline-Compatible STED Microscope for Combined Visible-Light and X-ray Studies of Biological Matter. J. Synchrotron Radiat. 26 (2019)

DOI: $10.1107 / \mathrm{S} 1600577519004089$

- Marten Bernhardt, Jan-David Nicolas, Markus Osterhoff, Haugen Mittelstädt, Matthias Reuss, Benjamin Harke, Andrew Wittmeier, Michael Sprung, Sarah Köster and Tim Salditt. Correlative Microscopy Approach for Biology using X-ray Holography, X-ray Scanning Diffraction and STED Microscopy. Nat. Commum. 9 (2018).

DOI: $10.1038 / \mathrm{s} 41467-018-05885-\mathrm{Z}$

- Manuela Denz, Gerrit Brehm, Clément Hémonnot, Heidi Spears, Andrew Wittmeier, Chiara Cassini, Oliva Saldanha, Eleonora Perego, Ana Diaz, Manfred Burghammer and Sarah Köster. Cyclic Olefin Copolymer as an X-ray Compatible Material for Microfluidic Devices. Lab Chip 18 (2018).

DOI: $10.1039 / \mathrm{c} 7 \mathrm{lc} 00824 \mathrm{~d}$ 
- Andrew Wittmeier, Andrew L. Holterhoff, Joel Johnson and John G. Gibbs. Rotational Analysis of Spherical, Optically Anisotropic Janus Particles by Dynamic Microscopy. Langmuir 31 (2015).

DOI: 10.1021/acs.langmuir.5b02864 